\title{
A Study of Neutrino Oscillations in MINOS
}

\author{
Tobias Martin Raufer \\ University College, Oxford
}
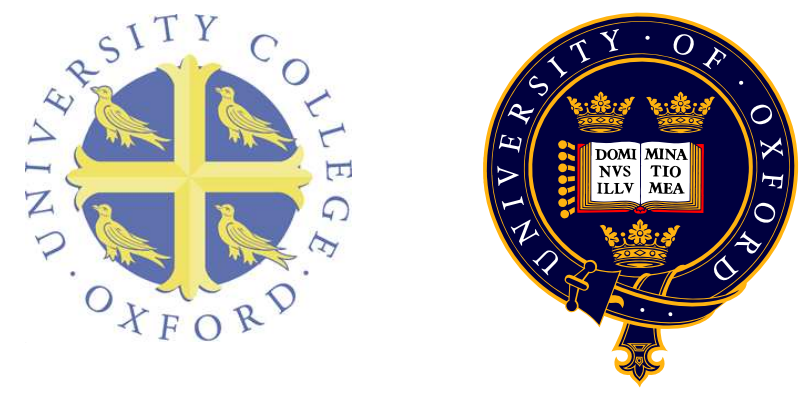

Thesis submitted in partial fulfilment of the requirements for the degree of Doctor of Philosophy at the University of Oxford

Trinity Term, 2007 


\title{
A Study of Neutrino Oscillations in MINOS
}

\author{
Tobias Martin Raufer \\ University College, Oxford \\ Thesis submitted in partial fulfilment of the requirements \\ for the degree of Doctor of Philosophy \\ at the University of Oxford. \\ Trinity Term, 2007
}

\begin{abstract}
MINOS is a long-baseline neutrino oscillations experiment located at Fermi National Accelerator Laboratory (FNAL), USA. It makes use of the NuMI neutrino beamline and two functionally identical detectors located at distances of $\sim 1 \mathrm{~km}$ and $\sim 735 \mathrm{~km}$ from the neutrino production target respectively. The Near Detector measures the composition and energy spectrum of the neutrino beam with high precision while the Far Detector looks for evidence of neutrino oscillations.

This thesis presents work conducted in two distinct areas of the MINOS experiment: analysis of neutral current and charged current interactions. While charged current events are only sensitive to muon neutrino disappearance, neutral current events can be used to distinguish oscillations into sterile neutrinos from those involving only active neutrino species.

A complete, preliminary neutral current study is performed on simulated data. This is followed by a more detailed investigation of neutral current neutrino interactions in the MINOS Near Detector. A procedure identifying neutral current interactions and rejecting backgrounds due to reconstruction failures is developed. Two distinct event classification methods are investigated. The selected neutral current events in the Near Detector are used to extract corrections to the neutral current cross-section in the MINOS Monte Carlo simulation as a function of energy. The resulting correction factors are consistent with unity.

The main MINOS charged current neutrino disappearance analysis is described. We present the Monte Carlo tuning procedure, event selection, extrapolation from Near to Far Detector and fit for neutrino oscillations. Systematic errors on this measurement are evaluated and discussed in detail. The data are consistent with neutrino oscillations with the following parameters: $2.74_{-0.26}^{+0.44} \times 10^{-3} \mathrm{eV}^{2}$ and $\sin ^{2}\left(2 \theta_{23}\right)>0.87$ at $68 \%$ confidence level.
\end{abstract}




\section{Acknowledgements}

A great many people have supported me on the long and arduous journey which eventually led to this thesis and the degree of D.Phil. It is now my pleasure to give them all my heartfelt thanks.

First and foremost, a huge thank you has to go to my supervisor Dr Alfons Weber for his continuous support and for letting me get on with things without continuously breathing down my neck. Without his help and encouragement, this thesis would simply not have come into existence.

Thanks also to my second supervisor Dr Giles Barr who took on the important role of chief motivator during the stressful final months and whose excellent proof reading made this thesis a lot more coherent and (hopefully) readable.

A big thank you goes to the Oxford MINOS group members, both past and present, for lots of interesting and fruitful discussions over coffee and lunch, and for generally making the department such a fun place to work at. I won't write down a long list of names but I need to mention one: my colleague and friend Phill Litchfield. We worked together during all my time at Oxford, and our many discussions as well as his persistent questioning undoubtedly improved this thesis immensely.

It is a great pleasure to take this opportunity to acknowledge the people who supported me financially and without whom I would never have been able to pursue this degree at Oxford. They are: the German Academic Exchange Service (DAAD), the Physics Department at the University of Oxford, the Gisela \& Erwin 
Sick Stiftung Waldkirch, and the Stadtwerke Waldkirch GmbH.

None of this would have happened without the love and encouragement from my parents. They believed in me all the way, even when it meant I was staying at university for nearly a decade. Thank you so much!

Finally, a very special thanks to Jasmin Kirchner for her love, support, and patience, especially during these last months when joy and despair appeared often very close together. 


\section{Contents}

1 Introduction 1

2 Neutrino Physics $\quad 4$

2.1 The Neutrino - A journey of 77 years . . . . . . . . . . . . . . . 4

2.2 Neutrino Oscillations . . . . . . . . . . . . . . . . . 6

2.2.1 Three-Family Neutrino Oscillations . . . . . . . . . . . . . . 9

2.2.2 Two-flavour approximation . . . . . . . . . . . . . . . 10

2.2.3 Sub-dominant oscillations . . . . . . . . . . . . . . 12

2.2.4 Neutrino oscillations in matter . . . . . . . . . . . . . . . 13

2.2.5 Sterile neutrinos . . . . . . . . . . . . . . 15

2.3 Experimental evidence . . . . . . . . . . . . . . . . . . 16

2.3.1 Solar neutrinos . . . . . . . . . . . . . . . . . 16

2.3.2 Atmospheric neutrinos . . . . . . . . . . . . . 23

2.3.3 Sterile neutrinos and the search for $\theta_{13} \ldots \ldots$. . . . . . 30

2.3.4 Summary of the experimental results . . . . . . . . . . . 32

2.4 Future experiments . . . . . . . . . . . . . . . . 33

3 The MINOS experiment 35

3.1 Overview of the experimental layout . . . . . . . . . . . . 35

3.2 The NuMI beam . . . . . . . . . . . . . . . . . . 36

3.3 Detector technology . . . . . . . . . . . . . . . . . . 39 
3.3.1 Detection principles . . . . . . . . . . . . . . . 40

3.3.2 Steel \& Magnet Coils . . . . . . . . . . . . . . . . . . . . . 40

3.3.3 The Scintillator system . . . . . . . . . . . . . . . . . 41

3.3.4 The photo-detectors ............... . . 43

3.4 The different MINOS detectors . . . . . . . . . . . . . . . . 44

3.4.1 The Calibration Detector . . . . . . . . . . . . . . . . 44

3.4 .2 The Far Detector . . . . . . . . . . . . . . . . 44

3.4.3 The Near Detector . . . . . . . . . . . . . . . . . 47

3.5 MINOS physics goals . . . . . . . . . . . . . . . . 52

3.6 MINOS data. . . . . . . . . . . . . . . . 54

3.6.1 Neutrino beam data . . . . . . . . . . . . . . 55

3.6.2 Monte Carlo data . . . . . . . . . . . . . . . . 56

3.7 MINOS event reconstruction . . . . . . . . . . . . . . 58

4 Preliminary sensitivity study: The 2005 Mock Data Challenge 61

4.1 Overview of the $\mathrm{NC}$ analysis procedure . . . . . . . . . . . 61

4.2 Event selection . . . . . . . . . . . . . . . . . . . . 62

4.2 .1 Fiducial cuts . . . . . . . . . . . . . . . 63

4.2.2 Selection Cuts . . . . . . . . . . . . . . . 64

4.3 Near detector fit . . . . . . . . . . . . . . . . . 67

4.4 Neutrino oscillation fit . . . . . . . . . . . . . . . . . 69

4.5 Results. . . . . . . . . . . . . . . . . . 72

4.5.1 Input values for the mock data set generation . . . . . . . 73

4.6 Conclusion . . . . . . . . . . . . . . . . . . . . . 74

5 Selecting Neutral Current neutrino interactions in the Near De$\begin{array}{ll}\text { tector } & 76\end{array}$

5.1 Backgrounds and "Data cleaning" . . . . . . . . . . . . 76

5.1 .1 Reconstruction failures . . . . . . . . . . . . 78 
5.1.2 An instrumental effect: PMT afterpulsing . . . . . . . . . 90

5.1 .3 CC interactions . . . . . . . . . . . . . . . . 94

5.2 Cut-based selection . . . . . . . . . . . . . . . . . 94

5.2 .1 Event selection cuts . . . . . . . . . . . . . . . 96

5.2.2 Resulting energy spectrum . . . . . . . . . . . . . 97

5.3 Artificial Neural Networks . . . . . . . . . . . . . . . . . . 98

5.3.1 Principle of the method . . . . . . . . . . . . 98

5.3.2 Implementation of neural networks for a Neutral Current event selection . . . . . . . . . . . . . . . 101

5.3 .3 Input variables . . . . . . . . . . . . . . . . . . . . 104

5.3.4 Performance of the ANN . . . . . . . . . . . . . . 105

5.4 Results . . . . . . . . . . . . . . . . . . . . 110

5.5 Conclusion . . . . . . . . . . . . . . . . . . . 111

6 NC Cross-section Measurement in the Near Detector 112

6.1 Overview of the analysis procedure . . . . . . . . . . . . 113

6.2 Data samples . . . . . . . . . . . . . . . . . . . . 115

6.3 The fitting technique . . . . . . . . . . . . . . . . 115

6.3 .1 Verifying the fit . . . . . . . . . . . . . 117

6.4 NC cross-section results . . . . . . . . . . . . . . . . . . 118

6.5 Systematic error evaluation . . . . . . . . . . . . . . . . 121

6.5.1 Effects considered . . . . . . . . . . . . . . . 122

6.5.2 Results . . . . . . . . . . . . . . . . . . 124

6.6 Conclusion . . . . . . . . . . . . . . . . . . . . . . . 129

$\begin{array}{lll}7 & \text { Muon neutrino disappearance analysis } & 131\end{array}$

7.1 Charged current event selection . . . . . . . . . . . . . . 132

7.2 Neutrino flux tuning . . . . . . . . . . . . . . . . . 135

7.3 Beam extrapolation to the Far Detector . . . . . . . . . . . . . 138 
7.4 Far Detector Oscillation Fit . . . . . . . . . . . . . . . . . 141

7.5 Oscillation result . . . . . . . . . . . . . . . . . . . . . . 142

7.6 Systematic error evaluation . . . . . . . . . . . . . . . . . . 142

7.6.1 Fake data study for individual systematic errors . . . . . . . 144

7.6.2 Including systematic errors in the fit . . . . . . . . . . . 153

7.7 Conclusion . . . . . . . . . . . . . . . . . . 155

8 Conclusion and Outlook 156

A Optimising electronics thresholds in the Far Detector

A.1 Far Detector front-end electronics . . . . . . . . . . . . . . . . 159

A.2 Optimising the dynode thresholds . . . . . . . . . . . . . . 160

A.2.1 Motivation for the tuning . . . . . . . . . . . . 160

A.2.2 A special run sequence: the dynode scan . . . . . . . . . . 161

A.2.3 Stability of the procedure . . . . . . . . . . . 163

A.2.4 Dynode threshold to ADC correspondence . . . . . . . . . 163

A.3 Summary . . . . . . . . . . . . . . . . . 166

Bibliography 


\section{List of Figures}

2.1 Neutrino mass hierarchies . . . . . . . . . . . . . . . 11

2.2 Coherent forward scattering diagrams . . . . . . . . . . . . . . . 14

2.3 Predicted SSM solar neutrino fluxes . . . . . . . . . . . . . . . . 18

2.4 SNO results . . . . . . . . . . . . . . . . . . 20

2.5 KamLAND prompt energy spectrum . . . . . . . . . . . . . 22

2.6 Solar neutrino oscillation contours . . . . . . . . . . . . . . . 23

2.7 Early atmospheric neutrino results . . . . . . . . . . . 25

2.8 Zenith angle distributions from Super-Kamiokande . . . . . . . . 26

2.9 Super-Kamiokande L/E analysis . . . . . . . . . . . . . . . . . 28

2.10 Allowed regions from atmospheric neutrino experiments . . . . . . . 29

2.11 LSND allowed region and MiniBooNE $90 \%$ C.l. . . . . . . . . . 31

3.1 Schematic of the MINOS experiment . . . . . . . . . . . 35

3.2 The major components of the NuMI beamline . . . . . . . . . 36

3.3 Different NuMI beam settings . . . . . . . . . . . . . . . . 38

3.4 Schematic of a single scintillator strip . . . . . . . . . . . . . . . 41

3.5 Attenuation in the wavelength shifting fibre . . . . . . . . . . . . 42

3.6 Scintillator modules in the Far Detector . . . . . . . . . . . . . 43

3.7 A frontal view of the fully installed Far Detector . . . . . . . . . . . 45

3.8 The Near Detector during plane installation . . . . . . . . . . . . . 48

3.9 The different sections of the Near Detector . . . . . . . . . . . . . . 49 
3.10 Plane layout in the Near Detector . . . . . . . . . . . . . . 50

3.11 Monte Carlo simulation of the MINOS sensitivity . . . . . . . . . . 52

3.12 MINOS sensitivity to $\theta_{13} \ldots \ldots \ldots \ldots$

3.13 Sterile neutrino signal _. . . . . . . . . . . . . . 54

3.14 Development of beam data acquisition . . . . . . . . . . . 55

3.15 MINOS event reconstruction . . . . . . . . . . . . . . 58

4.1 Event length $L$ distributions for Near and Far Detector Monte Carlo 64

4.2 Track extension $\epsilon$ distributions for Near and Far Detector Monte Carlo .......................... 65

4.3 Relative track fit error $\hat{\sigma}$ distributions for Near and Far Detector Monte Carlo . . . . . . . . . . . . . . . . . . . . . . 66

4.4 Selection efficiency and purity . . . . . . . . . . . . 67

4.5 Near Detector neutral current and charged current energy spectra . 70

4.6 Far detector energy spectra f . . . . . . . . . . . . . . 72

4.7 Confidence contours for the Mock Data Challenge . . . . . . . . . . 73

$5.1 \mathrm{x}-\mathrm{y}$ projection of fiducial event vertex positions $\ldots \ldots . . . . \quad 77$

5.2 Event completeness variable in Monte Carlo . . . . . . . . . . . . . 79

5.3 Example of a split event . . . . . . . . . . . . . . . . . . 80

$5.4 \min \Delta t$ and $\Delta z$ variables $\ldots \ldots \ldots$

$5.5 \Delta z$ vs. $\min \Delta t$ plots for low completeness events . . . . . . . . . . 82

5.6 Two different example "leakage" events . . . . . . . . . . . . . . . . 83

5.7 Steepness variable for events with $E_{\text {reco }}<5 \mathrm{GeV} \ldots . . . . . .85$

5.8 Veto regions . . . . . . . . . . . . . . . . . . . . . 86

5.9 Veto variables . . . . . . . . . . . . . . . . 86

5.10 Number of strips distribution . . . . . . . . . . . . . . . 87

$5.11 \mathrm{~N}-1$ plots for the cut variables . . . . . . . . . . . . . . 88

5.12 Neutral current energy spectra with and without cuts applied . . . 90 
5.13 Efficiencies and purities before and after applying cleaning cuts . . 91

5.14 Event pulse height and charge-weighted strip times in the spill window 93

5.15 Position of late activity in pixel space . . . . . . . . . . . . . . 93

$5.162 \mu s$-cut for afterpulsing . . . . . . . . . . . . 95

5.17 Selection variables for the simple selection . . . . . . . . . . . . 97

5.18 Selected neutral current spectrum . . . . . . . . . . . . . . 98

5.19 Schematic representation of a neuron . . . . . . . . . . . . 100

5.20 Schematic representation of the neural networks used . . . . . . 103

5.21 ANN input variables . . . . . . . . . . . . . . . . 106

5.22 ANN input variables (cont'd) . . . . . . . . . . . . 107

5.23 Neural network training . . . . . . . . . . . . . . . 108

5.24 ANN output distributions . . . . . . . . . . . . . . . . . . 109

5.25 ANN selected neutral current spectrum . . . . . . . . . . . . . 110

5.26 Comparison of selection efficiency and purity . . . . . . . . . . . 111

6.1 Neutral current spectrum contributions from different neutrino energy ranges . . . . . . . . . . . . . . . . . . . 114

6.2 Neutral current reconstructed energy spectra for different beams . . 116

6.3 Pull distributions . . . . . . . . . . . . . . . . . . . . . . 119

6.4 Best-fit reconstructed energy distributions . . . . . . . . . . . . . 120

6.5 ANN output distributions . . . . . . . . . . . . . . . . . 123

6.6 Systematically shifted spectra (LE) . . . . . . . . . . . . . 125

6.7 Systematically shifted spectra $(\mathrm{ME}) \ldots \ldots . . \ldots 126$

6.8 Systematically shifted spectra (HE) . . . . . . . . . . . . . 127

6.9 Systematic errors on scale parameters . . . . . . . . . . . . . . 128

7.1 PDF variables and PID parameter . . . . . . . . . . . 133

7.2 PID selection efficiencies and purities . . . . . . . . . . . . 135

7.3 CC energy spectra for different beam configurations . . . . . . . . . 137 
7.4 Sketch: operation of the Beam Matrix . . . . . . . . . . . . . 140

7.5 Beam Matrix predictions for systematically shifted Monte Carlo . . 141

7.6 Far Detector data and Monte Carlo energy spectra . . . . . . . . . 143

7.7 Allowed regions in the $\left(\Delta m_{32}^{2}, \sin ^{2}\left(2 \theta_{23}\right)\right)$ plane at $60,90,99 \%$ C.L. 143

7.8 Systematically shifted fake data sets . . . . . . . . . . . . . . 147

7.9 Systematically shifted fake data sets (cont'd) . . . . . . . . . . . 148

7.10 Ratio of FD prediction and fake data . . . . . . . . . . . . . 150

7.11 Ratio of FD prediction and fake data (cont'd) . . . . . . . . . 151

7.12 Approximate effect of systematic errors on allowed region . . . . . . 152

7.13 Allowed region with and without systematic errors . . . . . . . . . . 154

A.1 The VA front-end board . . . . . . . . . . . . . . . . 160

A.2 Trigger rate vs. dynode threshold . . . . . . . . . . . . . 161

A.3 Difference in determined dynode thresholds . . . . . . . . . . . . . 163

A.4 Singles ADC spectra for different dynode threshold settings . . . . . 164

A.5 ADC to dynode threshold correspondence . . . . . . . . . . 165 


\section{List of Tables}

2.1 Solar neutrino fluxes . . . . . . . . . . . . . . . . . . 17

3.1 Different beam configurations . . . . . . . . . . . . 56

5.1 Efficiencies and purities before and after data cleaning . . . . . . . 91

6.1 Near Detector cross-section fit: Systematic error evaluation . . . . . 124

7.1 Systematic parameters considered in the fake data study . . . . . 145

7.2 Best-fit parameter shifts for fake data sets . . . . . . . . . . . 149 


\section{Chapter 1}

\section{Introduction}

Neutrino physics has been among the most exciting topics in particle physics in recent years. We now know that neutrinos have non-zero masses and that neutrinos can change their flavour. Not only was neutrino flavour change observed in solar neutrino experiments, neutrino oscillations have also been shown to happen in terrestrial experiments.

MINOS ${ }^{1}$ is one of these terrestrial experiments. Based at Fermilab's Main Injector accelerator, MINOS measures an intense beam of neutrinos over a baseline of $735 \mathrm{~km}$. The neutrino beam is sampled by two large steel and scintillator calorimeters, one close to the beam production target at Fermilab and one in the Soudan mine in northern Minnesota.

MINOS measures neutrino oscillations by comparing the neutrino energy spectrum measured in the Far Detector to a predicted spectrum for the null hypothesis. To ensure the Far Detector prediction is as accurate as possible, the neutrino beam is measured with much higher statistics by the Near Detector at Fermilab. The Near Detector records thousands of neutrino interactions every day, making it possible to determine the neutrino flux and cross-section with very high precision.

This thesis is about neutrino oscillations in MINOS, studied in two somewhat

\footnotetext{
${ }^{1}$ MINOS stands for Main Injector Neutrino Oscillation Search
} 
distinct analyses. One analysis concerns itself with neutral current interactions in MINOS, the other one with charged current events. In the following paragraphs, a brief outline of what awaits the reader in the different chapters is given.

Chapter 2 introduces the necessary background in neutrino physics as a whole, both in theory and experiment. It starts with a brief journey through 77 years of exciting discoveries involving the neutrino before laying the theoretical foundations for neutrino oscillations. The formula describing the oscillation probability for neutrinos is derived and relevant approximations and sub-dominant effects are introduced. The final part of the chapter gives an overview of important experimental results in neutrino physics, providing the background in which MINOS operates.

Chapter 3 gives a detailed description of the MINOS experiment. The neutrino beamline is described first, followed by the detector technology employed by the two MINOS detectors. The chapter concludes with brief descriptions of the measurement goals of MINOS, the data and Monte Carlo samples in use and the event reconstruction chain.

In Chapter 4, the description of the neutral current analysis, referred to above, starts. A complete, preliminary sensitivity study including basic versions of neutral current event selection, Near Detector Monte Carlo tuning and an oscillation fit for sterile neutrinos is presented.

Chapter 5 examines the selection of neutral current events in more detail. A pre-selection algorithm dealing with reconstruction failures and problems arising from the high event multiplicity in the Near Detector is developed. Two separate event selection methods, one using a series of cuts and one making use of artificial neural network (ANN) techniques are developed, evaluated and contrasted.

Chapter 6 uses a neutral current event sample, achieved with the ANN method detailed in chapter 5, to extract neutrino cross-section information from the Near Detector data. This is done with a view to improve the Monte Carlo prediction 
for future Far Detector oscillation fits.

The final analysis chapter is chapter 7 . It describes the MINOS $\nu_{\mu}$ charged current disappearance analysis which was published in Phys. Rev. Lett. in 2006. Special emphasis is put on the evaluation of systematic uncertainties on the measured neutrino oscillation parameters.

The final chapter 8 gives the summary and conclusion of this thesis. The results are briefly summarised and their implications for MINOS and neutrino physics as a whole are discussed. 


\section{Chapter 2}

\section{Neutrino Physics}

\subsection{The Neutrino - A journey of 77 years}

The neutrino has come a long way since its inception in 1930. Proposed in Pauli's famous letter addressed to the "Liebe Radioaktive Damen und Herren" [1] as a desperate solution to the continuous energy spectrum in nuclear $\beta$-decays and the "wrong" angular momentum statistic for certain nuclei, neutrinos have come to be a fundamental ingredient to the standard model of particle physics and even neutrino masses and oscillations are now widely accepted by the scientific community.

In 1932, soon after Pauli's letter, Chadwick indeed discovered a neutral particle [2], but it turned out to be too heavy to be Pauli's proposed particle. Chadwick's particle is named neutron leaving Pauli's particle (which he had also called neutron) without a name. In 1933, Fermi proposed to instead call it neutrino, the "little neutral one". He took the neutrino hypothesis and built his theory of the weak interaction, which he published in 1934 [3]. In the same year, Bethe and Peierls showed that neutrino-matter cross-sections should be extremely small [4].

After these rapid developments in the early years, the world had to wait until 1956 for the first direct observation of neutrinos by Reines and Cowan [5]. They 
detected $\bar{\nu}$ via the inverse $\beta$-reaction in a tank of water and $\mathrm{CdCl}_{2}$, using an ingenious coincidence technique. After this, the journey of the neutrino picked up speed again. In 1958, Goldhaber determined the helicity of the neutrino [6] to be left-handed and in 1959, Davis showed that the anti-neutrinos measured by Reines and Cowan could be distinguished from neutrinos [7] because the former ones didn't react with ${ }^{37} \mathrm{Cl}$. Only three years later, an experiment by Lederman et. al. [8] at the AGS accelerator at Brookhaven detected a second species of neutrino: the $\nu_{\mu}$.

The following two decades saw a large number of scattering experiments, using both electrons and neutrinos to probe the quark structure of nucleons. This culminated in 1974 with the discovery of the second generation of quarks (via the $J / \Psi)[9,10]$, "drawing even" with the two flavours of neutrinos. Shortly before this, in 1973, the Gargamelle bubble chamber experiment at CERN discovered neutral current neutrino interactions [11], an important step towards electroweak unification.

In the meantime, Davis continued his solar neutrino experiment, which he started in the Homestake mine in 1968 [12]. From early on, there was a discrepancy between theoretical predictions and the measured solar neutrino flux. This discrepancy persisted and came to be known as the solar neutrino problem.

The 1980s saw three new experiments, trying to get to the bottom of the solar neutrino problem: GALLEX [13], SAGE [14] and Kamiokande [15]. The deficit stubbornly persisted. In 1985, Mikheyev and Smirnov [16] developed Wolfenstein's earlier work [17] on neutrino oscillations enhanced by the presence of matter: the MSW effect. Could the solar neutrino deficit be due to oscillations?

During the same decade, Kamiokande and another proton decay experiment called IMB [18] discovered another deficit: the atmospheric neutrino anomaly. This anomaly manifests itself in a zenith angle dependent departure from expectations for the flux of atmospheric neutrinos. In short, there are fewer neutrinos 
coming from below than from above.

In 1989, after only a few months of data taking with the new LEP accelerator, studies of the $Z$ boson lifetime revealed that there are three neutrino families $[19,20,21,22]$. It wasn't until 2001 however that the third neutrino, the $\nu_{\tau}$, was directly observed by the DONUT [23] experiment.

During the 1990s, both the solar and atmospheric neutrino deficits were confirmed by updated results from the various experiments.In 1998, Super-Kamiokande (Super-K), a larger version of the Kamiokande experiment, published their atmospheric neutrino results [24]. The evidence for neutrino oscillations as an explanation to the two anomalies was getting stronger.

One of the most exciting decades in neutrino physics however started with the turn of the millennium. After the direct observation of the $\nu_{\tau}$ in 2001 (mentioned above), the solar neutrino problem was finally solved by SNO [25] in 2002. This result was impressively confirmed in by the KamLAND [26] experiment using an accelerator neutrino beam. Neutrino oscillations were also confirmed to be the cause for the atmospheric neutrino anomaly: Updated Super-K measurements, the K2K [27] accelerator experiment and finally the first results from MINOS [28] leave no room for doubt anymore.

Neutrino oscillations in MINOS are the focus of this thesis. We will therefore discuss the theory of neutrino oscillations in the following section. The chapter will conclude with a detailed discussion of the experimental evidence, some of which has already been alluded to, and give an outline of the experimental program in the near future.

\subsection{Neutrino Oscillations}

Neutrino oscillations occur if two conditions are realised in nature: neutrinos are massive and there is leptonic mixing. In this section, we develop the formalism of 
neutrino oscillations from these two basic premises.

If neutrinos are massive, there exists a set of mass eigenstates denoted by $\nu_{i}$. The mass eigenstates diagonalise the free Hamiltonian and therefore describe the evolution of neutrinos in space and time. Interactions of neutrinos with matter on the other hand are described by the flavour eigenstates $\nu_{\alpha}$. These states are eigenstates of the weak interaction which appear at the $W$ interaction vertex together with the charged lepton partners $l_{\alpha}$. Mixing means that the $\nu_{i}$ and the $\nu_{\alpha}$ are not identical.

The flavour eigenstates can be written as a superposition of the mass eigenstates:

$$
\left|\nu_{\alpha}\right\rangle=\sum_{i=1}^{n} U_{\alpha i}^{*}\left|\nu_{i}\right\rangle,
$$

where $n$ is the number of neutrino states and $U$ is the unitary leptonic mixing matrix, also known as PMNS matrix after Pontecorvo, Maki, Nakagawa and Sakata $[29,30]$. In the standard model $n=3$, but the derivation in this section holds for an arbitrary number of neutrino states.

The time evolution of the state $\left|\nu_{\alpha}\right\rangle$ is found by first considering the time evolution of the component mass eigenstates. Solving the Schrödinger equation for $\nu_{i}$, we obtain:

$$
\left|\nu_{i}(t)\right\rangle=e^{-i\left(E_{i} t-p_{i} L\right)}\left|\nu_{i}(0)\right\rangle
$$

where $\mathrm{L}$ is the distance travelled during the time interval t. Assuming all components $\nu_{i}$ have a common energy $\mathrm{E}$ and taking $E>>m_{i}$, we expand $p_{i}=$ $\sqrt{E^{2}-m_{i}^{2}} \approx E+m_{i}^{2} / 2 E$. Since neutrinos are ultra-relativistic, we can take $t \approx L$ and thus the Eq. 2.2 simplifies to

$$
\left|\nu_{i}(t)\right\rangle=e^{-i\left(m_{i}^{2} / 2 E\right) L}\left|\nu_{i}(0)\right\rangle
$$

A more rigorous approach treating the neutrinos as wave packets and thus avoiding 
the "common energy" assumption has been shown to lead to the same result [31]. In order to obtain an expression for the flavour eigenstate $\left|\nu_{\alpha}\right\rangle$ after travelling a distance $L$, we insert Eq. 2.3 into Eq. 2.1. This gives

$$
\left|\nu_{\alpha}(L)\right\rangle=\sum_{i=1}^{n} U_{\alpha i}^{*} e^{-i\left(m_{i}^{2} / 2 E\right) L}\left|\nu_{i}\right\rangle
$$

We invert Eq. 2.1, using the unitarity of $U$, and insert the result into Eq. 2.4. This replaces the mass eigenstates $\left|\nu_{i}\right\rangle$ on the right-hand side with a superposition of flavour eigenstates:

$$
\left|\nu_{\alpha}(L)\right\rangle=\sum_{\beta=1}^{n}\left[\sum_{i=1}^{n} U_{\alpha i}^{*} e^{-i\left(m_{i}^{2} / 2 E\right) L} U_{\beta i}\right]\left|\nu_{\beta}\right\rangle .
$$

The process of neutrino flavour change is evident in this equation. After propagating a distance $L$, the $\nu_{\alpha}$ becomes a superposition of all flavours. We can now compute the probability of measuring the original $\nu_{\alpha}$ as being of flavour $\nu_{\beta}$, by finding the square of the matrix element $\left\langle\nu_{\beta} \mid \nu_{\alpha}(L)\right\rangle$ :

$$
\begin{aligned}
P\left(\nu_{\alpha} \rightarrow \nu_{\beta}\right) & =\left|\left\langle\nu_{\beta} \mid \nu_{\alpha}(L)\right\rangle\right|^{2} \\
& =\sum_{i, j} U_{\alpha i}^{*} U_{\beta i} U_{\alpha j} U_{\beta j}^{*} e^{-i \Delta m_{i j}^{2} L / 2 E},
\end{aligned}
$$

where $\Delta m_{i j}^{2}=m_{i}^{2}-m_{j}^{2}$ and $i$ and $j$ iterate over the mass eigenstates in the theory. Explicitly separating the right-hand side into its real and imaginary parts, the $\sin ^{2}$ dependence corresponding to neutrino oscillations becomes visible:

$$
\begin{aligned}
P\left(\nu_{\alpha} \rightarrow \nu_{\beta}\right)=\delta_{\alpha \beta} & -4 \sum_{i>j} \Re\left(U_{\alpha i}^{*} U_{\beta i} U_{\alpha j} U_{\beta j}^{*}\right) \sin ^{2}\left(\frac{\Delta m_{i j}^{2} L}{4 E}\right) \\
& +2 \sum_{i>j} \Im\left(U_{\alpha i}^{*} U_{\beta i} U_{\alpha j} U_{\beta j}^{*}\right) \sin ^{2}\left(\frac{\Delta m_{i j}^{2} L}{2 E}\right) .
\end{aligned}
$$

Here, $\delta_{\alpha \beta}$ is the Kronecker symbol. 
Aside: CP violation A complex PMNS matrix $U$ implies $C P$ violation in the lepton sector. Consider the operation of $C P T$ on the oscillation probability for anti-neutrinos, $P\left(\overline{\nu_{\alpha}} \rightarrow \overline{\nu_{\beta}}\right)$ :

$$
P\left(\bar{\nu}_{\alpha} \rightarrow \bar{\nu}_{\beta}\right) \stackrel{C P T}{=} P\left(\nu_{\beta} \rightarrow \nu_{\alpha}\right)=P\left(\nu_{\alpha} \rightarrow \nu_{\beta} ; U \rightarrow U^{*}\right)
$$

The second equality can be found, for example, by looking carefully at Eq. 2.7. This means that, assuming CPT invariance holds, $U \neq U^{*}$ implies different oscillation probabilities for neutrinos and anti-neutrinos and thus leptonic $C P$ violation.

From here on, we need to consider more specific theories. In the following section, the phenomenology of the standard model scenario with three distinct neutrino states is described.

\subsubsection{Three-Family Neutrino Oscillations}

In the case of three flavour (or mass) states, the PMNS mixing matrix is given explicitly by:

$$
U_{P M N S}=\left(\begin{array}{ccc}
U_{e 1} & U_{e 2} & U_{e 3} \\
U_{\mu 1} & U_{\mu 2} & U_{\mu 3} \\
U_{\tau 1} & U_{\tau 2} & U_{\tau 3}
\end{array}\right)
$$

In an analogous fashion to the CKM quark mixing matrix [32], the PMNS matrix can be parameterised using three angles $\theta_{i j}$ and a phase $\delta$ :

$$
U=\left(\begin{array}{ccc}
c_{12} c_{13} & s_{12} c_{13} & s_{13} e^{-i \delta} \\
-s_{12} c_{23}-c_{12} s_{23} s_{13} e^{i \delta} & c_{12} c_{23}-s_{12} s_{23} s_{13} e^{i \delta} & s_{23} c_{13} \\
s_{12} s_{23}-c_{12} c_{23} s_{13} e^{i \delta} & -c_{12} s_{23}-s_{12} c_{23} s_{13} e^{i \delta} & c_{23} c_{13}
\end{array}\right)
$$

where $c_{i j} \equiv \cos \left(\theta_{i j}\right)$ and $s_{i j} \equiv \sin \left(\theta_{i j}\right)$. As shown in the previous section, a nonzero phase $\delta$ implies $\mathrm{CP}$ violation in the lepton sector. If neutrinos are Majorana 
particles ${ }^{1}$, there are an additional two phase factors which multiply the diagonal of the matrix and are therefore unobservable in neutrino oscillations. Consequently, these phases are ignored here. They do however play a role in neutrino-less double beta decay [33].

It is instructive to decompose the PMNS matrix even further to show the product of three distinct rotation matrices:

$$
U=\left(\begin{array}{ccc}
1 & & \\
& c_{23} & s_{23} \\
& -s_{23} & c_{23}
\end{array}\right)\left(\begin{array}{cccc}
c_{13} & & s_{13} e^{-i \delta} \\
& 1 & \\
s_{13} e^{i \delta} & & & c_{13}
\end{array}\right)\left(\begin{array}{ccc}
c_{12} & s_{12} & \\
-s_{12} & c_{12} & \\
& & 1
\end{array}\right)
$$

where the $\delta$-phase has been grouped with the central $\theta_{13}$-matrix. This decomposition is useful to visualise the impact of the various neutrino experimental programmes: the first rotation is measured by atmospheric and long-baseline accelerator neutrino experiments, the third one by solar neutrino experiments. The centre matrix contains the as yet unmeasured third mixing angle $\theta_{13}$ and the $\delta$ phase. These latter two parameters are the goal of current and future long-baseline accelerator and reactor experiments.

In fact, these three sectors decouple and can thus be treated in isolation by the relevant experiments. This is a consequence of the smallness of the mixing angle $\theta_{13}$, which makes the central matrix in Eq. 2.11 nearly diagonal, and the very different sizes of the two mass splittings $\Delta m_{12}^{2}$ and $\Delta m_{13}^{2}$. This will be explained in more detail in the following section.

\subsubsection{Two-flavour approximation}

Figure 2.1 shows the two possible neutrino mass hierarchies for the three-neutrino case. They are referred to as the normal (left) and inverted (right) hierarchy

\footnotetext{
${ }^{1} \mathrm{~A}$ particle type for which particle and antiparticle are identical is referred to as a Majorana particle. This is only possible for neutral particles like neutrinos.
} 


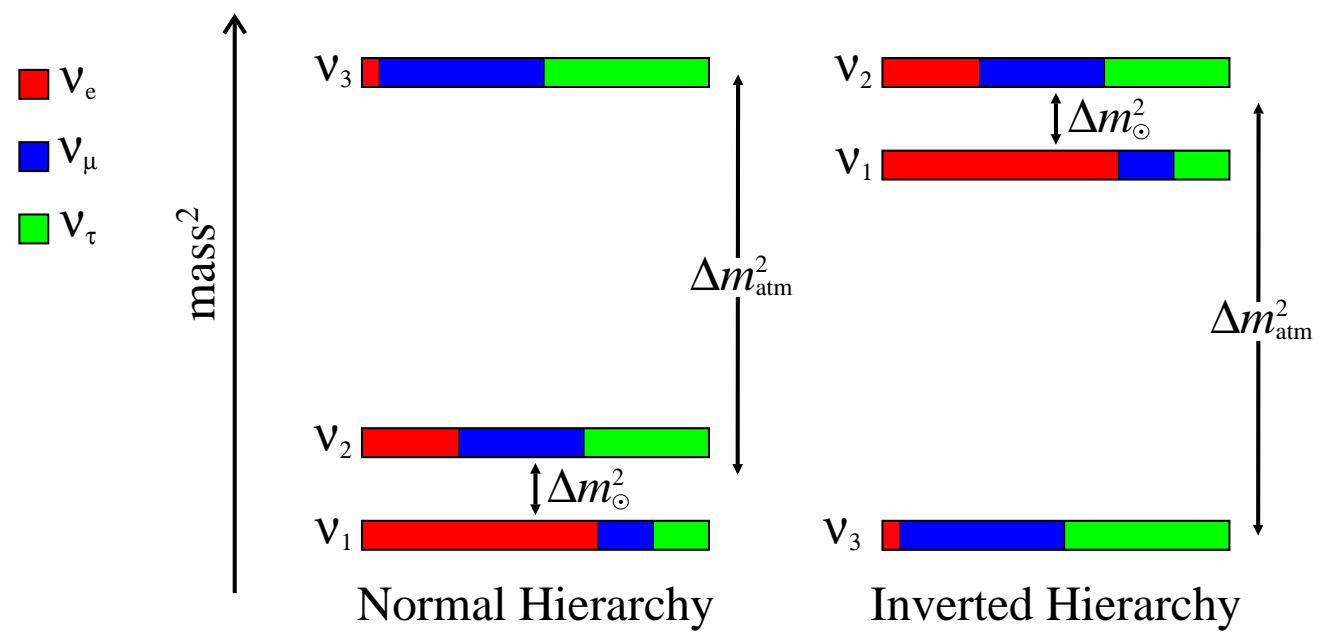

Figure 2.1: Neutrino mass hierarchies for the normal (left) and inverted (right) case. The colours show the approximate flavour content of each mass state, according to the latest experimental results (see section 2.3).

respectively. These are the only two possibilities since the sign of $\Delta m_{12}^{2}$ is known from experiment ${ }^{2}$. The various experiments in recent years (see section 2.3) have furthermore established that $\Delta m_{13}^{2} \gg \Delta m_{12}^{2}$.

Eq. 2.7 shows that the effect of neutrino oscillations becomes sizable if the term $\Delta m_{i j}^{2} L / E$ is $O(1)$. The quantities $L$ and $E$ depend on the experimental setup. In accelerator experiments for example, $L$ is a constant determined by the location of the detector(s). Given the size difference in the two mass splittings, it follows that only one $\Delta m^{2}$ matters for a given experiment. This means that it is justified in many cases to analyse the data in terms of a two-neutrino approximation. This has been called "quasi-two-neutrino oscillations" or "one mass scale dominance" $[34]$.

With this justification in mind, we simplify Eq. 2.7. In the case of two neutrino generations, there is only one squared mass difference $\Delta M^{2}$ and one mixing angle $\theta$. Since there are no more degrees of freedom, $\mathrm{CP}$ violation is not possible in this

\footnotetext{
${ }^{2}$ The reason for this is given in section 2.2.4.
} 
simple framework. The PMNS matrix is simply a 1-dimensional rotation:

$$
U=\left(\begin{array}{cc}
\cos \theta & \sin \theta \\
-\sin \theta & \cos \theta
\end{array}\right)
$$

Expressing $\Delta M^{2}$ in units of $e V^{2}, L$ in $\mathrm{km}$, and $E$ in $\mathrm{GeV}$, and inserting the so far omitted factors of $\hbar$ and $c$, the oscillation probability becomes:

$$
P\left(\nu_{\alpha} \rightarrow \nu_{\beta}\right)=\sin ^{2}(2 \theta) \sin ^{2}\left(1.27 \frac{\Delta M^{2} L}{E}\right)
$$

In the MINOS experiment, the dominant mass splitting is the atmospheric mass splitting $\Delta m_{32}^{2}$ and the main oscillation channel is $\nu_{\mu} \rightarrow \nu_{\tau}$. In this case, Eq. 2.13 becomes:

$$
P\left(\nu_{\mu} \rightarrow \nu_{\tau}\right)=\sin ^{2}\left(2 \theta_{23}\right) \sin ^{2}\left(1.27 \frac{\Delta m_{32}^{2} L}{E}\right)
$$

\subsubsection{Sub-dominant oscillations}

When discussing the parameterisation of the PMNS matrix in Eq. 2.11, we pointed out that $\theta_{13}$ was known to be small and the central matrix therefore close to diagonal. The smallness of this angle compared to the other mixing angles $\theta_{12}$ and $\theta_{23}$ makes its oscillations small in comparison with solar and atmospheric oscillations and hence sub-dominant. In order to measure a non-zero angle $\theta_{13}$ in an experiment, these sub-dominant oscillation modes have to be explored. In MINOS, this is done via $\nu_{e}$ appearance in the $\nu_{\mu}$ beam.

Writing Eq. 2.7 for $\nu_{\mu} \rightarrow \nu_{e}$ oscillations and keeping only leading order terms, we obtain the oscillation probability:

$$
\mathrm{P}\left(\nu_{\mu} \rightarrow \nu_{e}\right) \simeq \sin ^{2}\left(\theta_{23}\right) \sin ^{2}\left(2 \theta_{13}\right) \sin ^{2}\left(\frac{1.27 \Delta m_{32}^{2} L}{E}\right)
$$

The effect is suppressed by a factor of $\sin ^{2}\left(2 \theta_{13}\right)$ compared to Eq. 2.14. Note that 
the measurement of $\theta_{13}$ also depends on the value of the atmospheric mixing angle $\theta_{23}$ which is not preceded by a factor of two here.

The parameterisation given in Eq. 2.11 shows that, to observe $C P$ violation in the lepton sector, $\theta_{13}$ needs to be non-zero. Otherwise the off-diagonal terms in the central matrix are zero and it reduces to the identity.

The effect of a non-zero phase $\delta$ and thus $C P$ violation enters into $\mathrm{P}\left(\nu_{\mu} \rightarrow \nu_{e}\right)$ as a sub-leading term. A full three-flavour expression for the $\nu_{\mu} \rightarrow \nu_{e}$ oscillation probability, including the effect of the $\delta$-phase, is for example given in [35].

\subsubsection{Neutrino oscillations in matter}

So far in this chapter, the neutrinos are assumed to propagate freely, i.e. through vacuum, on their way from source to detector. In many cases, this assumption is not justified.

It was first pointed out by Wolfenstein [17], and later by Mikheyev and Smirnov [16], that the presence of matter can strongly affect neutrino oscillation probabilities. The propagation of neutrinos through matter is affected by coherent forward scattering off electrons, which can proceed via either a charged current (CC) or neutral current (NC) reaction (see Figure 2.2). Since incoming and outgoing particles must be the same, only $\nu_{e}$ can take part in the $\mathrm{CC}$ reaction, whereas all flavours $\nu_{\alpha}$ are affected by NC scattering. This gives rise to an additional potential in the Hamiltonian which depends on the density of the matter traversed by the neutrinos. This is known as the MSW effect.

For $\nu_{e}$ the additional potential due to the ambient matter is $V=\sqrt{2} G_{F} N_{e}$, where $G_{F}$ is the Fermi coupling constant and $N_{e}$ is the electron density. Terms common to all flavours have been neglected since they only result in an overall phase factor.

A detailed derivation of matter effects for different conditions is beyond the 

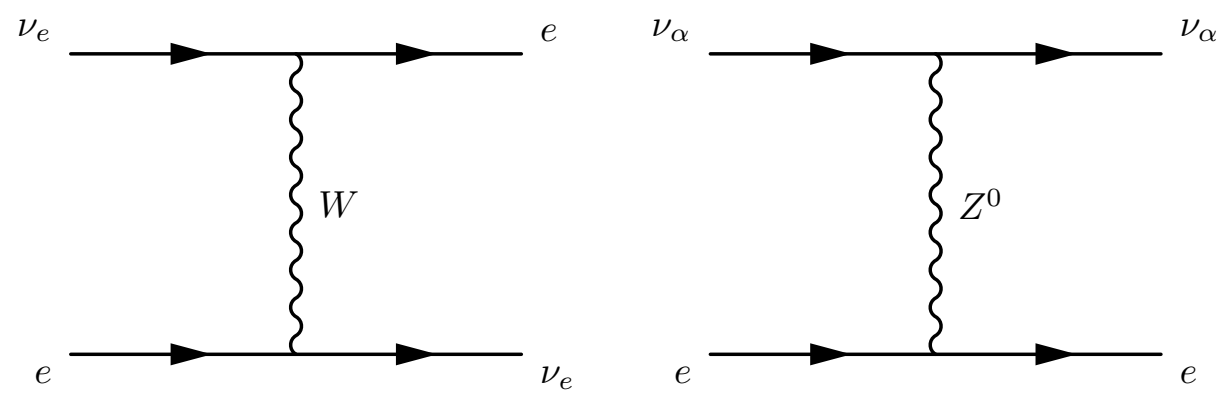

Figure 2.2: Coherent forward scattering diagrams for neutrinos going through matter. The charged current (CC) process (left) can only occur for $\nu_{e}$ whereas neutral current $(\mathrm{NC})$ scattering affects all flavours $\nu_{\alpha}$ equally. This introduces an additional potential to the propagation Hamiltonian.

scope of this thesis. Instead, we quote the results for constant matter density along the neutrino flight path (from [36]). This assumption is justified in the case of MINOS since the path of the neutrino beam only traverses the Earth's crust. In this case, the oscillation probability takes the same form as given in Eq. 2.14 with an effective mixing angle $\theta_{M}$

$$
\sin ^{2} 2 \theta_{M}=\frac{\sin ^{2} 2 \theta}{\left(\cos 2 \theta-\frac{2 E}{\Delta m_{32}^{2}} \sqrt{2} G_{F} N_{e}\right)^{2}+\sin ^{2} 2 \theta}
$$

and an effective mass splitting

$$
\left(\Delta m_{32}^{2}\right)_{M}=\Delta m_{32}^{2} \sqrt{\sin ^{2} 2 \theta+\left(\cos 2 \theta-\frac{2 E}{\Delta m_{32}^{2}} \sqrt{2} G_{F} N_{e}\right)^{2}} .
$$

The denominator of Eq. 2.16 contains a resonance condition, when $\cos 2 \theta=$ $\frac{2 E}{\Delta m_{32}^{2}} \sqrt{2} G_{F} N_{e}$. That means even a small vacuum mixing angle can cause large mixing due to the MSW effect. It should also be noted that the resonance condition contains the sign of $\Delta m_{32}^{2}$. This means, matter effects distinguish between the two possible hierarchies shown in Figure 2.1.

If the density of matter varies along the neutrinos' path, things become more complicated and can lead to a multitude of effects. An example for the MSW 
effect with varying densities along the flight path is neutrino oscillations in the sun. Neutrinos produced in nuclear reactions in the core of the sun travel through a decreasing matter density profile until reaching the surface. In the case of solar neutrinos, the MSW effect is the dominant contribution to the observed oscillation. This is the reason why the sign of $\Delta m_{12}^{2}$ is known from experiment.

\subsubsection{Sterile neutrinos}

The number of light active neutrinos, i.e. neutrinos coupling to the $Z$ boson, has been measured very precisely to be $2.9840 \pm 0.0082$ by the LEP experiments [37]. There is however a long standing experimental problem, sometimes called the LSND [38] anomaly. The LSND experiment at Los Alamos National Laboratory observed $\bar{\nu}_{e}$ appearance in a $\bar{\nu}_{\mu}$ beam over a short baseline, consistent with a mass splitting of $\Delta m^{2} \sim 1 \mathrm{eV}^{2}$. That means that more than two different mass splittings, and therefore more than 3 neutrino states, are needed to explain all the evidence from the various neutrino oscillation experiments (to be discussed in section 2.3). This problem has recently been alleviated somewhat by the non-observation reported by the MiniBooNE collaboration [39], but the LSND observation is not completely ruled out yet.

More than two mass splittings can be introduced without violating the constraint from the $Z$ pole by introducing one or more light "sterile" neutrinos. Sterile neutrinos do not take part in any standard model interactions but do take part in oscillations. Eq. 2.1 can in principle contain an arbitrary number of neutrino species, as long as only three combinations result in an active neutrino coupling to the $Z$. For a more detailed treatment on PMNS matrices with sterile neutrinos see $[40]$.

The MINOS experiment can search for sterile neutrinos by measuring neutral current interactions, the detection of which is one of the main topics of this thesis. 
Oscillations into sterile neutrinos would result in a measurable deficit of neutral current events. Conversely, if only active neutrinos were involved in oscillations, the neutral current spectrum would remain unchanged.

\section{$2.3 \quad$ Experimental evidence}

The body of experimental evidence for neutrino oscillations gathered over the decades can only be described as compelling. Experimental results reported during the last decade from the big water Cerenkov experiments Super-Kamiokande (Super-K) and SNO were able to explain two long standing puzzles in neutrino physics: the solar neutrino problem and the atmospheric neutrino anomaly. Neutrino oscillations are now universally accepted as the solution to these puzzles.

\subsubsection{Solar neutrinos}

The sun is powered by the nuclear fusion processes of hydrogen burning, producing an intense flux of electron neutrinos. The combined effect of these solar fusion processes can be written as follows:

$$
4 p+2 e^{-} \rightarrow{ }^{4} \mathrm{He}+2 \nu_{e}+26.73 \mathrm{MeV} .
$$

Some of the released energy is carried by the neutrinos, with an average of $\left\langle E_{\nu}\right\rangle \sim$ $0.6 \mathrm{MeV}$. A breakdown into the different reactions is shown in Table 2.1, taken from [41]. A graphical representation is given in Figure 2.3. The theoretical fluxes and errors come from the latest version of the Standard Solar Model (SSM) by Bahcall et. al. [42], whose prediction is in excellent agreement with helio-seismological measurements [43]. 


\begin{tabular}{lll}
\hline \hline Reaction & Abbr. & Flux $\left(\mathrm{cm}^{-2} \mathrm{~s}^{-1}\right)$ \\
\hline$p p \rightarrow d e^{+} \nu$ & $p p$ & $5.99(1.00 \pm 0.01) \times 10^{10}$ \\
$p e^{-} p \rightarrow d \nu$ & $p e p$ & $1.42(1.00 \pm 0.02) \times 10^{8}$ \\
${ }^{3} \mathrm{He} p \rightarrow{ }^{4} \mathrm{He} e^{+}$ & $h e p$ & $7.93(1.00 \pm 0.16) \times 10^{3}$ \\
${ }^{7} \mathrm{Be} e^{-} \rightarrow{ }^{7} \mathrm{Li} \nu+(\gamma)$ & ${ }^{7} \mathrm{Be}$ & $4.84(1.00 \pm 0.11) \times 10^{9}$ \\
${ }^{8} \mathrm{~B} \rightarrow{ }^{8} \mathrm{Be}^{*} e^{+} \nu$ & ${ }^{8} \mathrm{~B}$ & $5.69(1.00 \pm 0.16) \times 10^{6}$ \\
${ }^{13} \mathrm{~N} \rightarrow{ }^{13} \mathrm{C} e^{+} \nu$ & ${ }^{13} \mathrm{~N}$ & $3.07\left(1.00_{-0.28}^{+0.31}\right) \times 10^{8}$ \\
${ }^{15} \mathrm{O} \rightarrow{ }^{15} \mathrm{~N} e^{+} \nu$ & ${ }^{15} \mathrm{O}$ & $2.33\left(1.00_{-0.29}^{+0.33}\right) \times 10^{8}$ \\
${ }^{17} \mathrm{~F} \rightarrow{ }^{17} \mathrm{O} e^{+} \nu$ & ${ }^{17} \mathrm{~F}$ & $5.84(1.00 \pm 0.52) \times 10^{6}$ \\
\hline \hline
\end{tabular}

Table 2.1: Solar neutrino fluxes from the different solar fusion reactions.

\section{Radio-chemical experiments}

Radio-chemical measurements of the solar neutrino flux were pioneered by Davis et. al. [12] in the Homestake mine, South Dakota. The Homestake experiment, starting in the late 1960s, made use of the reaction

$$
{ }^{37} \mathrm{Cl}+\nu_{e} \rightarrow{ }^{37} \mathrm{Ar}+e^{-}
$$

with chlorine in the form of 390,000 litres of $\mathrm{C}_{2} \mathrm{Cl}_{4}$. The capture reaction on chlorine has an energy threshold of $0.841 \mathrm{MeV}$. The resulting argon was extracted in a helium purging and filtration process and subsequently counted in a proportional counter, using its decay with a 35-day half-life. From early on, it was realised that the measured flux of solar neutrinos was significantly below the SSM predictions. This deficit was later confirmed by the gallium experiments SAGE [14] and GALLEX [13] and by the water Čerenkov experiments Kamiokande [44] and Super-Kamiokande (Super-K) [45]. This solar neutrino problem, born in the Homestake experiment, persisted for three decades.

The gallium experiments are conceptually similar to the Homestake experiment. By replacing chlorine with Gallium, they achieve a lower detection energy threshold of $0.224 \mathrm{MeV}$ and thus become sensitive to neutrinos from the dominant 


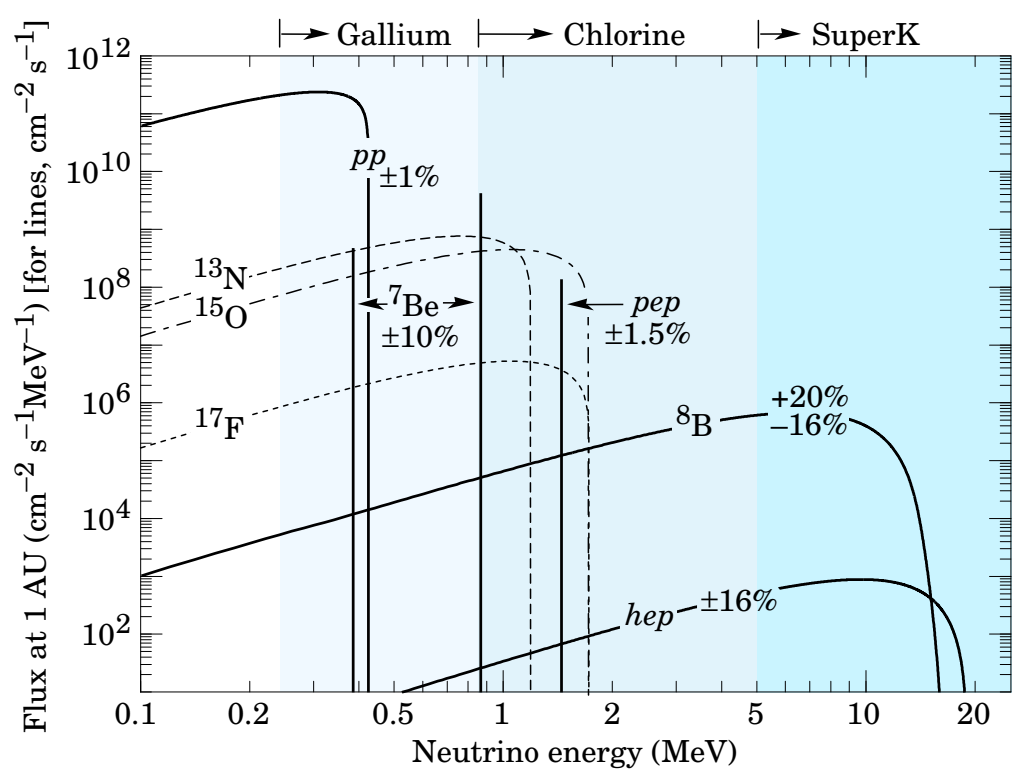

Figure 2.3: The solar neutrino energy spectrum predicted by the SSM. The energy ranges measurable by each of the main solar neutrino experiments are illustrated by the three coloured energy bands. Graph from Ref. [41].

$p p$-reaction. This means that much smaller detector volumes were sufficient to detect the solar neutrino flux. The detection reaction in the gallium experiments is:

$$
\nu_{e}+{ }^{71} \mathrm{Ga}=e^{-}+{ }^{71} \mathrm{Ge}^{*} .
$$

\section{Water Čerenkov experiments}

Water Čerenkov experiments are large tanks of water making use of the Čerenkov effect [46] as their detection reaction. Particles exceeding the speed of light in water create a "shock-wave" of photons which are measured by an array of photomultiplier tubes (PMTs) mounted on the walls of the tank. Neutrino experiments using water Čerenkov technology are Kamiokande, Super-K and, most recently, the Sudbury Neutrino Observatory (SNO) [47].

Kamiokande and its successor Super-K are located $1000 \mathrm{~m}$ underground in the Kamioka mine in Japan. They consist of a cylindrical tank filled with $680 \mathrm{t}$ and 
$22.5 \mathrm{kt}$ of water in the fiducial volume respectively. They measure neutrinos using the electron recoil in the elastic scattering reaction:

$$
\nu_{x}+e^{-} \rightarrow \nu_{x}+e^{-}
$$

where $x=e, \mu, \tau$. Although being sensitive to all three flavours via the neutral current (NC) interaction, the elastic scattering reaction is dominated by the $\nu_{e}$ flux, since both NC and CC interactions contribute in this case. The detection thresholds in Kamiokande and Super-K are $7 \mathrm{MeV}$ and $5 \mathrm{MeV}$ respectively. This means the two experiments are only sensitive to the higher energy ${ }^{8} \mathrm{~B}$ and hep neutrinos.

The most recent water Cerenkov experiment to be built is SNO. SNO is a spherical, $1 \mathrm{kt}$ ultra-pure heavy water detector located at a depth of $6010 \mathrm{~m}$ water equivalent in a mine in Sudbury, Canada. A transparent vessel containing the heavy water $\mathrm{D}_{2} \mathrm{O}$ is viewed from the outside by $\sim 9500$ PMTs mounted on a larger spherical vessel. The whole arrangement is immersed in ultra-pure light water providing shielding from natural radioactivity.

SNO detects the ${ }^{8} \mathrm{~B}$ neutrinos via three distinct reactions:

$$
\begin{array}{ll}
\mathrm{CC}: & \nu_{e}+d \rightarrow p+p+e^{-} \\
\mathrm{NC}: & \nu_{x}+d \rightarrow p+n+\nu_{x} \\
\mathrm{ES}: & \nu_{x}+e^{-} \rightarrow \nu_{x}+e^{-}
\end{array}
$$

The main advantage of SNO over other solar neutrino experiments is its ability to measure the total solar neutrino flux via the $\mathrm{NC}$ reaction. The final states of the $\mathrm{NC}$ and $\mathrm{CC}$ reactions in $\mathrm{D}_{2} \mathrm{O}$ are different, allowing the simultaneous measurement of the total and $\nu_{e}$-only fluxes. The NC reaction is detected via neutron capture.

SNO took data in three consecutive phases which only differed by the means 


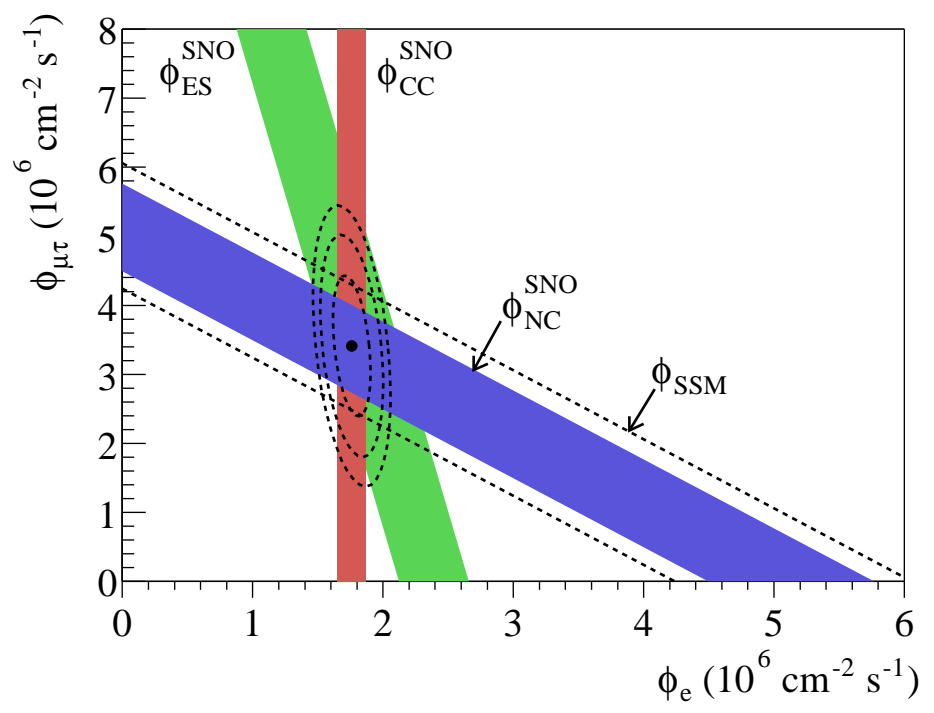

Figure 2.4: Results from SNO phase I for the CC, NC and ES detection reactions. The results are given in terms of the flux of ${ }^{8} B$ solar neutrinos separated into electron-like $\left(\phi_{e}\right)$ and non-electron-like $\left(\phi_{\mu \tau}\right)$ fluxes. The three measurements are consistent and in good agreement with the predictions from the SSM.

employed for the neutron detection in the NC reaction. In phase I, the neutron was captured on deuterium resulting in a distinctive $6.25 \mathrm{MeV} \gamma$-ray. In phase II, salt was added to increase the neutron capture efficiency, resulting in an $8.6 \mathrm{MeV}$ $\gamma$-cascade following the capture on $C l$. In phase III, the neutrons were detected by ${ }^{3} \mathrm{He}$ proportional counters.

Results from SNO's first running phase in terms of $\nu_{\mu \tau}$ vs. $\nu_{e}$ fluxes are shown in Figure 2.4. The three coloured bands show the results from the three detection reactions. They are both consistent with each other and with the predictions from the SSM, shown by the dotted lines. The measured fluxes, given in units of $10^{6} \mathrm{~cm}^{-2} \mathrm{~s}^{-1}$ are:

$$
\begin{aligned}
& \phi_{C C}=1.68_{-0.06}^{+0.06}(\text { stat. })_{-0.09}^{+0.08}(\text { syst. }) \\
& \phi_{E S}=2.35_{-0.22}^{+0.22}(\text { stat. })_{-0.15}^{+0.15}(\text { syst. }) \\
& \left.\left.\phi_{N C}=4.94_{-0.21}^{+0.21} \text { (stat. }\right)_{-0.34}^{+0.38} \text { (syst. }\right)
\end{aligned}
$$




\section{Scintillator detectors}

The Kamioka Liquid Anti-Neutrino Detector (KamLAND) [26] is a $1 \mathrm{kt}$ liquid scintillator detector located in the former Kamiokande cavern in the Kamioka mine. Although its experimental programme includes the measurement of ${ }^{7} \mathrm{Be}$ and ${ }^{8} \mathrm{~B}$ neutrinos from the sun, it is discussed in this section because of its ability to measure the anti-neutrino flux from 16 nuclear power reactors in Japan and South Korea. Assuming CPT invariance, this measurement covers the same oscillation parameter space as the previously discussed solar neutrino experiments.

KamLAND is located between 100 and $1000 \mathrm{~km}$ from the different nuclear reactors, but the majority of the $\nu_{e}$ flux reaching the KamLAND detector originates from reactors approximately $180 \mathrm{~km}$ away, resulting in a reasonable $L / E$ resolution. KamLAND measures the $\bar{\nu}_{e}$-flux via the inverse $\beta$-decay reaction:

$$
\bar{\nu}_{e}+p \rightarrow e^{+}+n
$$

The coincidence of the prompt annihilation signal from the positron and the delayed $2.2 \mathrm{MeV} \gamma$-ray from neutron capture is a powerful tool to discriminate against backgrounds from natural radioactivity.

KamLAND reports a flux of reactor $\bar{\nu}_{e}$ of $0.658 \pm 0.044$ (stat.) \pm 0.047 (syst.) of the expected value for no oscillations [48]. The distortion of the $\bar{\nu}_{e}$ energy spectrum is shown in Figure 2.5. An energy-dependent deficit of events, the signature of neutrino oscillations, is clearly visible.

\section{Neutrino oscillations interpretation of solar neutrino experiments}

Over three decades, solar neutrino experiments reported a deficit in the solar neutrino flux compared to theoretical predictions. Many theories explaining this deficit, e.g. neutrino decay [49] or decoherence [50], or were proposed during this period. It was not until the results from SNO were published, that neutrino 


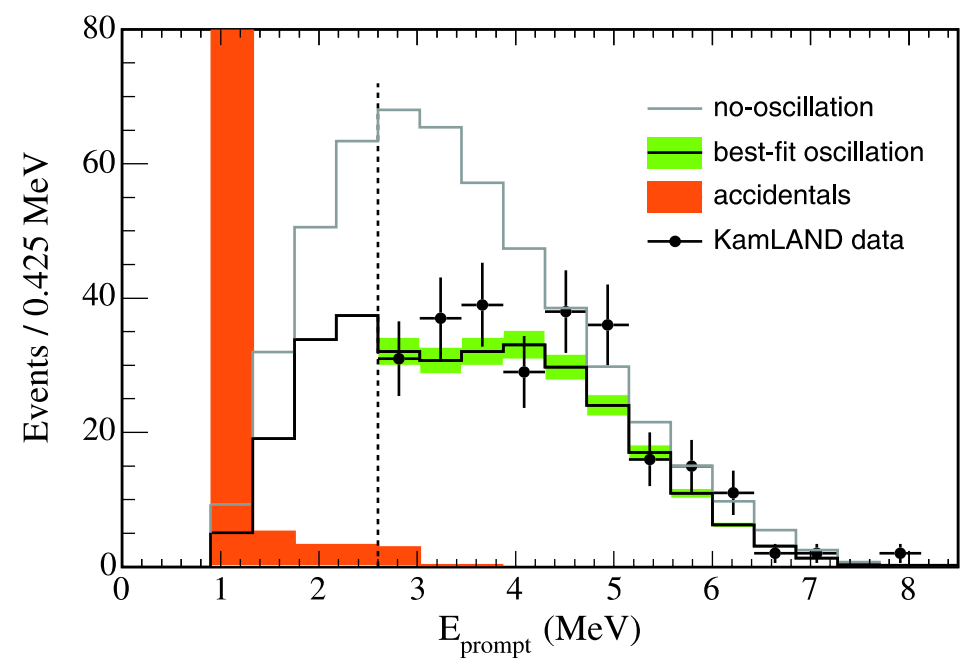

Figure 2.5: Prompt energy spectrum of reactor anti-neutrino events observed in KamLAND (figure from [48]). The measured and expected prompt energy spectra of anti-neutrino candidate events, along with the best-fit oscillation scenario are shown. The dashed line shows the position of the $2.6 \mathrm{MeV}$ analysis threshold.

oscillations became generally accepted as the solution to the solar neutrino problem and the correct description of neutrino propagation.

With its three complementary detection channels, SNO showed that the measured deficit is indeed due to neutrino flavour transitions and that the total flux of neutrinos from the sun is consistent with solar models.

KamLAND with its relatively short baseline and thus independence from matter effects significantly constrained the possible values of the squared mass difference $\Delta m_{12}^{2}$. In addition, the spectral distortion of the measured energy spectrum provided clear evidence for neutrino oscillations.

A combined fit to the data from all solar neutrino experiments plus KamLAND [48] yields the following best-fit oscillation parameters:

$$
\begin{aligned}
\Delta m_{12}^{2} & =7.9_{-0.5}^{+0.6} \times 10^{-5} \mathrm{eV}^{2} \\
\tan ^{2}\left(\theta_{12}\right) & =0.40_{-0.07}^{+0.10}
\end{aligned}
$$

at $68 \%$ C.L. A graphical representation of the parameter space is shown in Figure 

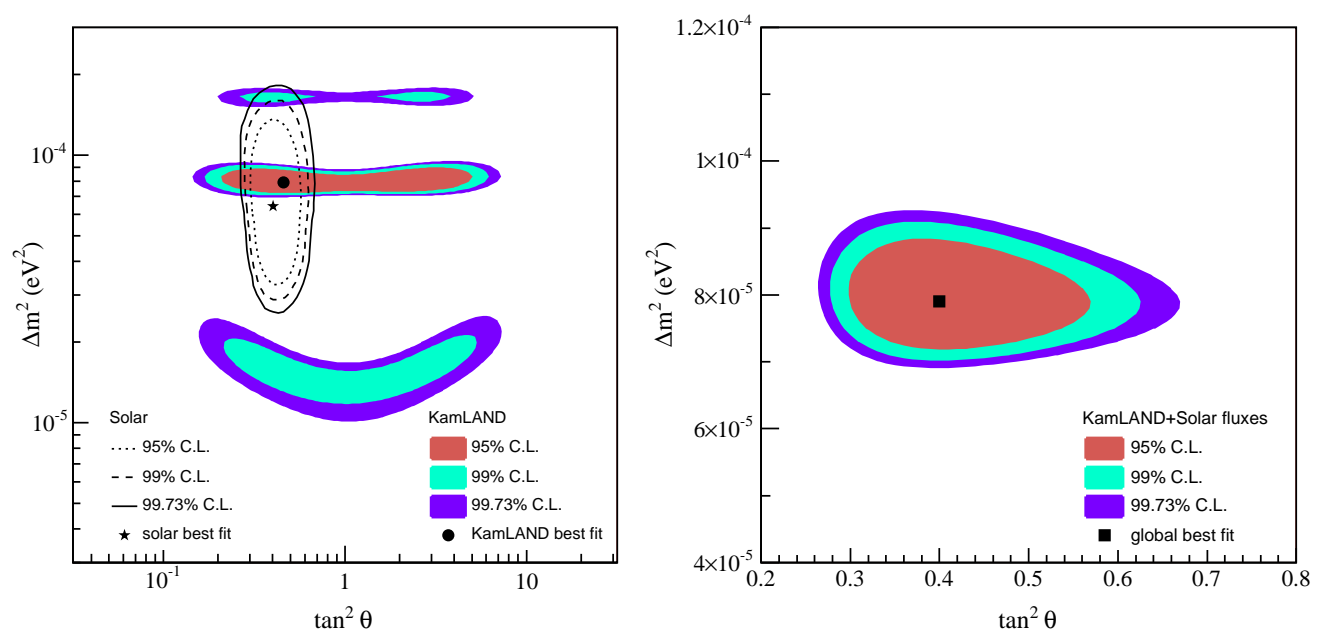

Figure 2.6: Solar neutrino oscillation allowed regions for $\theta_{12}$ and $\Delta m_{12}^{2}$. In (a), the shaded regions show the KamLAND results and the lines show the combined results of all solar neutrino experiments. Figure (b) shows the combined Solar+KamLAND allowed region. The global best-fit point is identified by a square.

2.6 (taken from [48]). Figure 2.6 (a) shows the result from all solar neutrino experiments and the KamLAND allowed regions separately, whereas in Figure 2.6 (b) the combined Solar+KamLAND result is shown. It is evident how the KamLAND result restricts the allowed region significantly.

\subsubsection{Atmospheric neutrinos}

The atmospheric neutrino anomaly was a term used to describe the observed deficit in the flux of $\nu_{\mu}$ created in the atmosphere compared to theoretical expectations. It was seen in proton decay experiments, for which the atmospheric neutrino flux was an important source of backgrounds.

High energy cosmic rays interacting with nucleons in the upper atmosphere (at an average altitude of $20 \mathrm{~km}$ ) create hadronic showers, mainly consisting of $\pi$ - and $K$-mesons. These mesons subsequently decay to produce neutrinos. The $\pi$-meson 
decay chain, for example, can be written as follows:

$$
\begin{aligned}
& \pi^{ \pm} \rightarrow \mu^{ \pm}+\nu_{\mu}\left(\bar{\nu}_{\mu}\right) \\
& \mu^{ \pm} \rightarrow e^{ \pm}+\nu_{e}\left(\bar{\nu}_{e}\right)+\nu_{\mu}\left(\bar{\nu}_{\mu}\right) .
\end{aligned}
$$

This means, the expected ratio of neutrino flavours $R=\frac{\nu_{\mu}+\bar{\nu}_{\mu}}{\nu_{e}+\bar{\nu}_{e}}$ from this decay chain is approximately 2. This calculation approximately holds for secondaries $\lesssim 2 \mathrm{GeV}$. At higher energies, the intermediate muons strike the Earth's surface before decaying, thus increasing $R$. Detailed calculations of the atmospheric neutrino flux $([51,52])$ involve the treatment of many effects, such as the composition and energy spectrum of the primary cosmic rays, the Earth's magnetic field and the density and composition of the atmosphere. Due to these complications, experimental data is usually presented as the double ratio $R^{\prime}$ defined as:

$$
R^{\prime}=\frac{R_{D A T A}}{R_{M C}}
$$

where $R_{D A T A}$ is the flavour ratio measured in experiment and $R_{M C}$ is the predicted number from Monte Carlo simulations.

The proton decay experiments IMB [53], a large water Čerenkov experiment, and Kamiokande (described in the previous section) first observed a significant deficit of muon-like events. IMB originally did not rule out the possibility of an unaccounted for systematic error, e.g. in the calculation of the $\nu$-flux. Kamiokande however soon presented much stronger evidence, quoting a value of $R^{\prime}=0.59 \pm$ 0.07 (stat.) and claiming to have rejected all known sources of systematic errors. This observed deficit was subsequently confirmed by other experiments. Figure 2.7 shows a summary of these results (taken from [54]). 


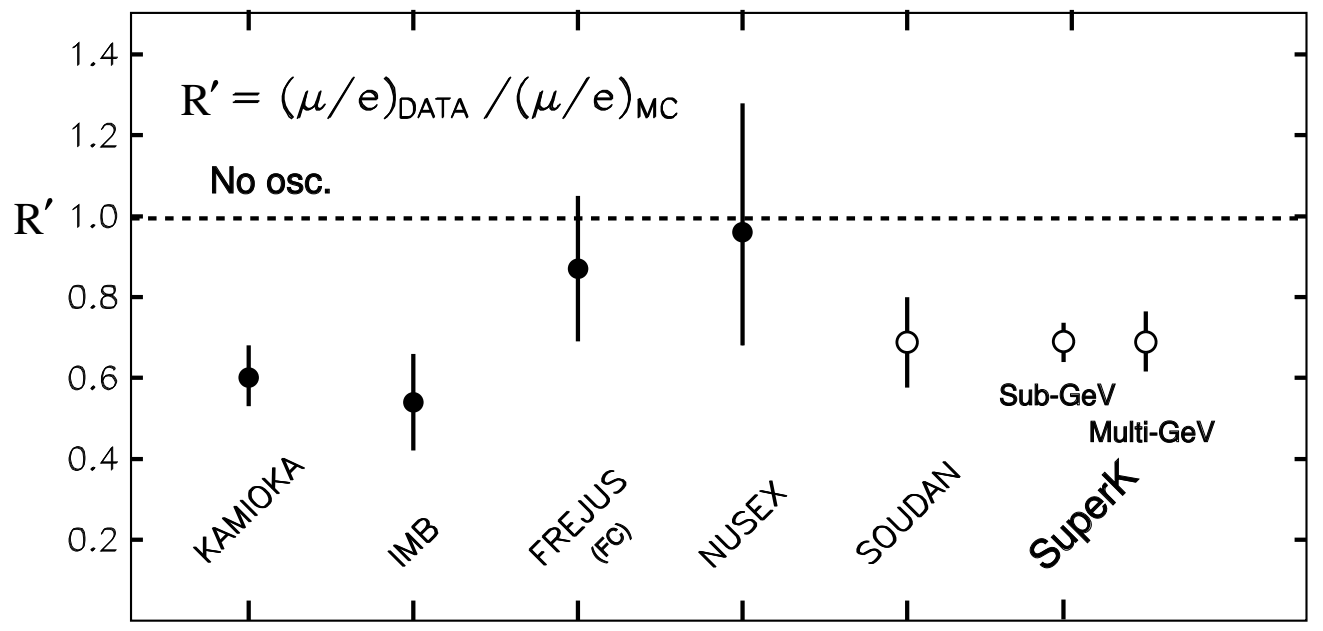

Figure 2.7: Atmospheric neutrino flux measurements from several experiments. $R^{\prime}$ is the ratio of ratios $R^{\prime}=R_{D A T A} / R_{M C}$. Plot from Ref. [54].

\section{Super-Kamiokande atmospheric neutrino results}

The most convincing evidence that the atmospheric neutrino anomaly is caused by neutrino oscillations was presented by the Super-Kamiokande experiment [24] (which was introduced in the previous section).

Atmospheric neutrinos have vastly different path lengths $L$, depending on whether they reach the detector from above or from below. Neutrinos reaching the detector from directly below have travelled the whole diameter of the earth whereas the neutrinos from above only traversed $\sim 20 \mathrm{~km}$. Super-K measures the zenith angle of neutrino events from the angle of the scattered electron or muon, and is thus sensitive to the distance travelled by the neutrino. Events are separated into e-like and $\mu$-like, depending on the quality of the Cerenkov ring.

Figure 2.8 shows the angular distributions of neutrino events for e-like and $\mu$-like events, separately for low energy and high energy events. In the $\mu$-like event samples, a clear zenith-angle dependent depletion is observed. Moreover, the differences between low energy and high energy events are strong evidence for the characteristic $L / E$ dependence of neutrino oscillations. The number of e-like events however is well described by the atmospheric neutrino Monte Carlo. 

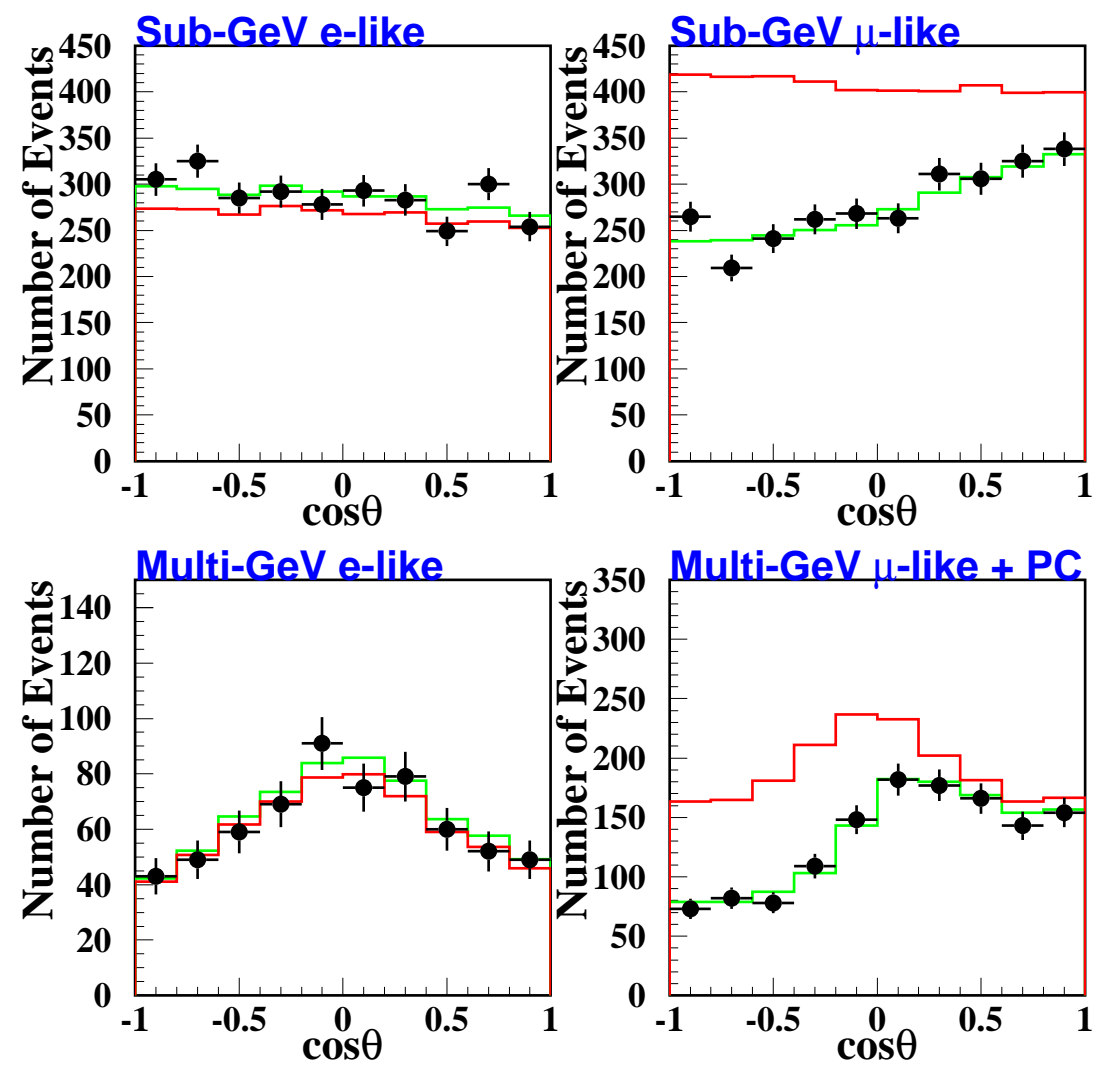

Figure 2.8: Zenith angle distributions from Super-Kamiokande (from [55]). The top figures show low energy, the bottom two high energy events, for e-like (left) and $\mu$-like (right) events respectively. The black dots show the data, the red line the Monte Carlo prediction without oscillations, and the green line the best-fit for oscillations. 
These observations are consistent with $\nu_{\mu} \rightarrow \nu_{\tau}$ oscillations. The latest zenithangle measurements analysed in the two-flavour approximation [56] result in the following parameters:

$$
\begin{gathered}
2 \times 10^{-3} \mathrm{eV}^{2} \leqslant \Delta m^{2} \leqslant 3 \times 10^{-3} \mathrm{eV}^{2} \\
\sin ^{2}(2 \theta)>0.93
\end{gathered}
$$

at $90 \%$ C.L.

In 2004, Super-Kamiokande published a dedicated L/E analysis [57], to provide even stronger evidence for the neutrino oscillations explanation of the atmospheric neutrino anomaly. Events with particularly good resolution in $L / E$ were selected in order to observe the $L / E$ dependence of the $\nu_{\mu}$-flux. The resulting ratio of observed $\mu$-like events over expectation, given in Figure 2.9, shows an indication of the characteristic oscillation "dip". The best-fit parameters for this analysis are consistent with those obtained from the zenith-angle measurements. The allowed regions in parameter space is shown in Figure 2.10 after discussing other atmospheric neutrino experiments.

\section{Other atmospheric neutrino experiments}

The atmospheric neutrino results from Super-Kamiokande have been independently verified by a number of other experiments. The Soudan2 [58] and MACRO [59] experiments are both large underground tracking calorimeters, located at the Soudan Underground Laboratory, USA and the Gran Sasso National Laboratory, Italy. Their results, together with the Super-K allowed regions, are shown in Figure 2.10.

The K2K experiment follows another approach to measuring atmospheric neutrino parameters. It is an accelerator based oscillation search, using the KEK neutrino beam and Super-K as its detector. K2K has a baseline of $250 \mathrm{~km}$ and 


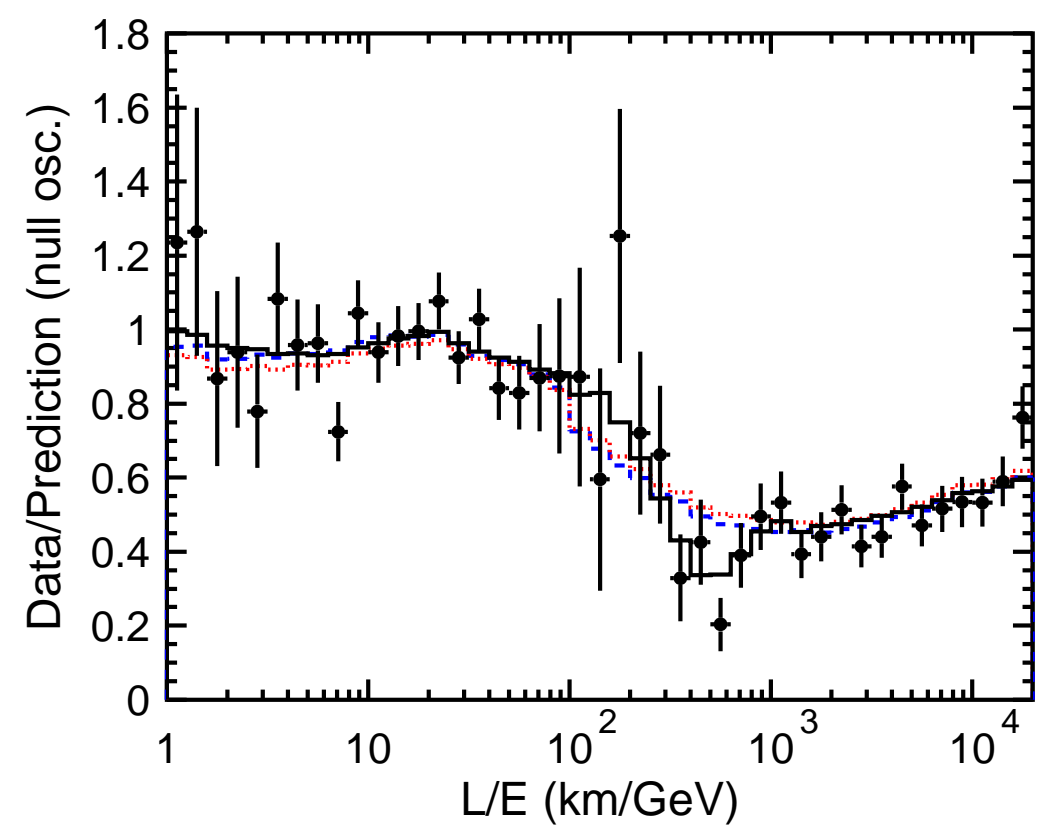

Figure 2.9: Ratio of observed over expected $\mu$-like events as a function of $L / E$, measured by Super-Kamiokande. The black dots show the data and the black line shows the best fit to oscillations. The coloured lines show the alternative theoretical scenarios neutrino decay (red) and decoherence (blue).

mean neutrino energy $\langle E\rangle$ of $1.3 \mathrm{GeV}$, thus probing the mixing parameters of the atmospheric sector.

With a final exposure of $0.92 \times 10^{20}$ protons-on-target, K2K obtained the following results [27]: $112 \nu_{\mu}$ events were observed compared to $158.1_{-8.6}^{+9.2}$ expected for no oscillations. The no-oscillations hypothesis is disfavoured at $4.3 \sigma$ and the observations are consistent with the oscillation parameters measured by Super-K. The allowed region in parameter space is shown, together with other atmospheric neutrino experiments, in Figure 2.10.

The MINOS experiment, which is the subject of this thesis, follows a very similar approach to the $\mathrm{K} 2 \mathrm{~K}$ experiment. It has a 3-times longer baseline of $735 \mathrm{~km}$ and an average beam energy of $\sim 3.5 \mathrm{GeV}$. The MINOS concept and experimental details will be explained in detail in Chapter 3. 


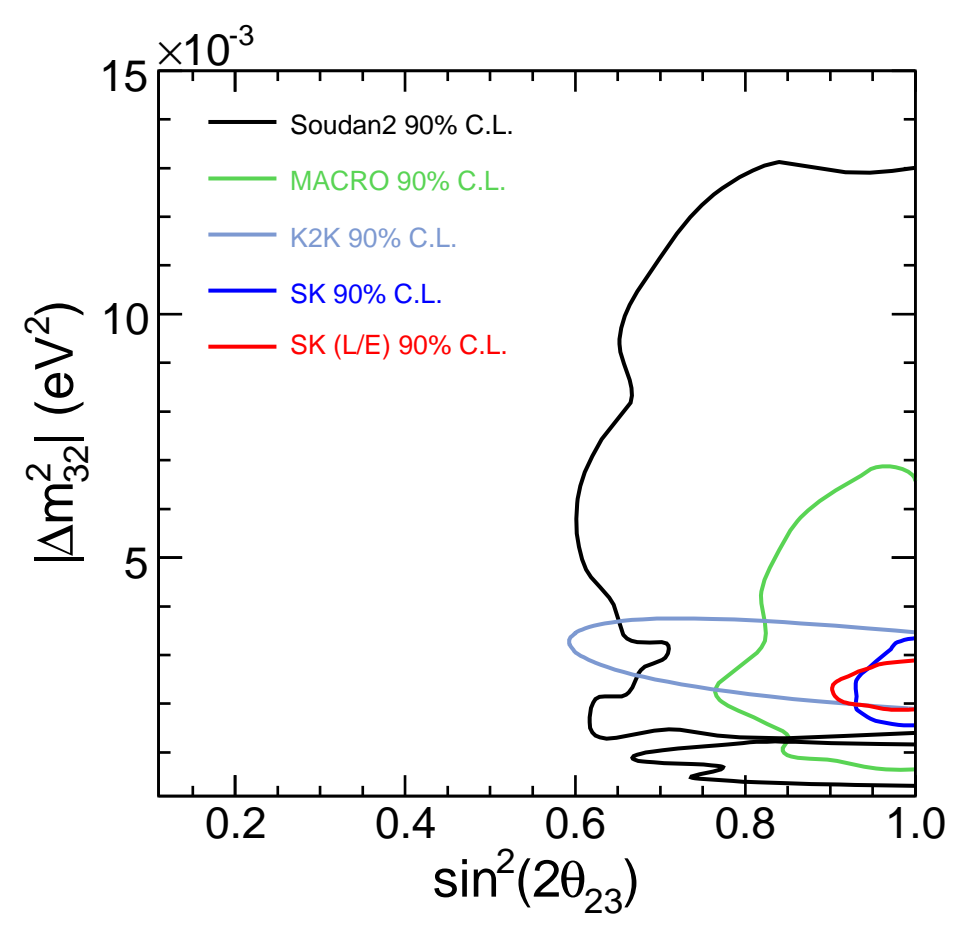

Figure 2.10: Allowed regions at $90 \%$ C.L. from atmospheric neutrino experiments (Soudan2, MACRO, Super-K and K2K). All experiments shown are consistent with the Super-K region. 


\subsubsection{Sterile neutrinos and the search for $\theta_{13}$ \\ Sterile neutrinos: LSND and MiniBooNE}

As explained in section 2.2.5 there is another anomaly in neutrino physics. The results from the LSND experiment point to neutrino oscillations with a large mass splitting of order $\sim 1 \mathrm{eV}^{2}$ which cannot be reconciled with the precision measurements from other neutrino experiments, both solar and atmospheric. When introducing additional sterile neutrinos, the observations can be made to agree.

The MiniBooNE experiment at Fermilab set out to independently verify or disprove the LSND observation. It uses neutrinos from the Booster beam line with a peak energy of $700 \mathrm{MeV}$ and a spherical liquid scintillator Čerenkov detector, located at a distance of $\sim 500 \mathrm{~m}$ from the target. With this combination of $L / E$, MiniBooNE is sensitive to the parameter space allowed by the LSND result.

MiniBooNE recently announced their first results [39], which rules out most but not all of the LSND parameter space. Their results are shown in Figure 2.11. From a two-neutrino analysis, neutrino appearance is ruled out as an explanation to the LSND anomaly at $98 \%$ C.L.

The sterile neutrino solution is not yet "dead" however. In fact, it has been recently pointed out that the results of LSND and MiniBooNE can be made to agree perfectly by introducing two sterile neutrinos [60]. MINOS will search for sterile neutrinos using the disappearance of neutral current events in the Far Detector.

\section{Search for $\theta_{13}$}

$\theta_{13}$ is the last remaining unmeasured angle in the PMNS matrix. Limits obtained from reactor experiments only show that it is small; it could potentially be zero. The current best limit on $\theta_{13}$ comes from the CHOOZ reactor experiment.

CHOOZ measured the disappearance of $\bar{\nu}_{e}$ produced in two nuclear power reactors in the north of France using a 5-ton gadolinium loaded liquid scintillator 


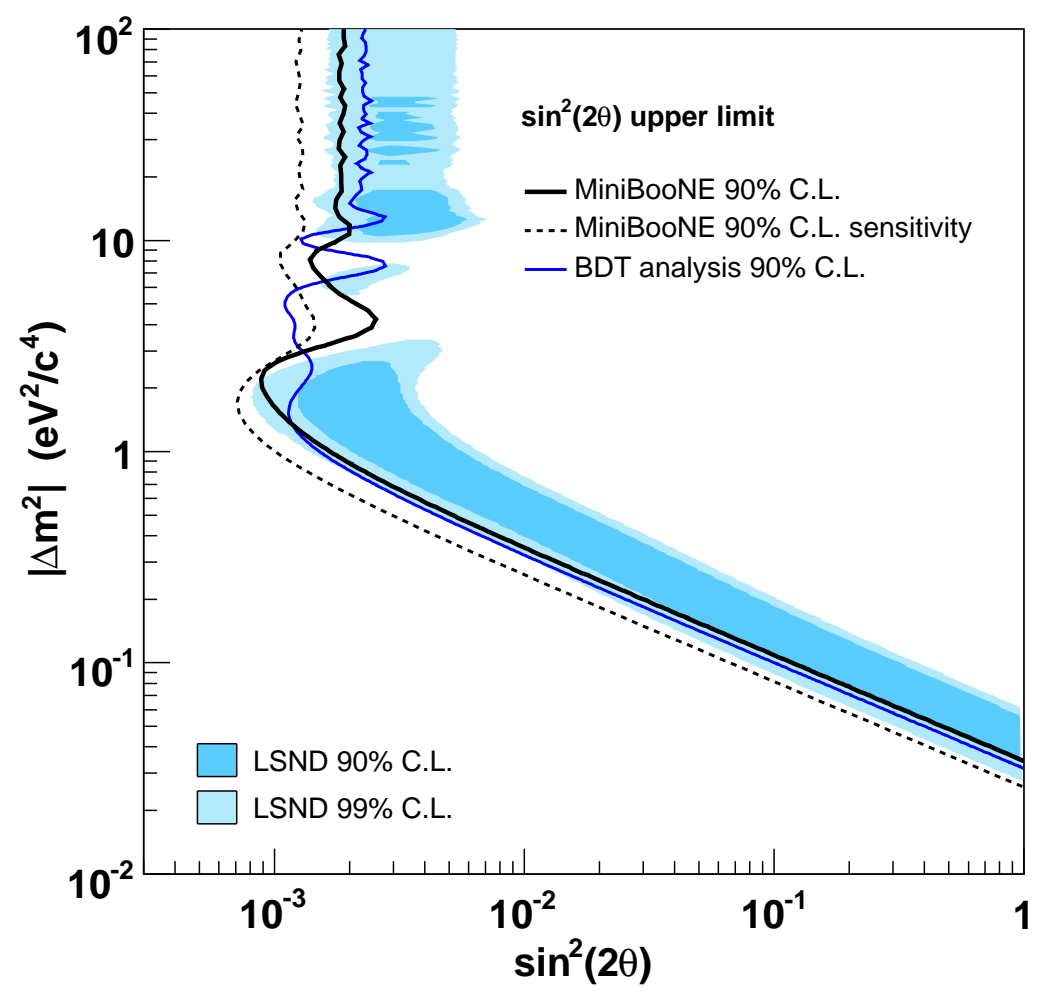

Figure 2.11: LSND allowed region and MiniBooNE $90 \%$ C.L. The MiniBooNE $90 \%$ C.L. exclusion limit is shown as a solid black line, compared with the LSND allowed region in dark blue. The dashed line shows the MiniBooNE sensitivity as determined from Monte Carlo. The blue line shows an alternative analysis method employed by MiniBooNE. MiniBooNE rules out most of the LSND parameter space. 
detector located $\sim 1 \mathrm{~km}$ from the reactor cores. The CHOOZ result is consistent with no $\bar{\nu}_{e}$ disappearance at $90 \%$ C.L [61]. This enables them to place an upper limit on the third mixing angle $\theta_{13}$. In general, this limit is a function of the atmospheric mass difference $\Delta m_{32}^{2}$. In the $\Delta m_{32}^{2}$ region suggested by the Super-K and $\mathrm{K} 2 \mathrm{~K}$ experiments, $\mathrm{CHOOZ}$ places a limit on $\theta_{13}$ of:

$$
\sin ^{2}\left(2 \theta_{13}\right)<0.15
$$

at $90 \%$ C.L.

\subsubsection{Summary of the experimental results}

So far in this section, we have given a broad overview of the many different experiments which shaped the field of neutrino physics in general, and neutrino oscillations in particular. We have traced the major steps and given the key results of these experiments.

In order to summarise the current knowledge of neutrino oscillations, we quote the global fit values for the different parameters ${ }^{3}$, as taken from [62]:

$$
\begin{aligned}
\Delta m_{21}^{2} & =(7.9 \pm 0.3) \times 10^{-5} \mathrm{eV}^{2} \\
\left|\Delta m_{31}^{2}\right| & =2.5_{-0.25}^{+0.20} \times 10^{-3} \mathrm{eV}^{2} \\
\sin ^{2}\left(\theta_{12}\right) & =0.30_{-0.03}^{+0.02} \\
\sin ^{2}\left(\theta_{23}\right) & =0.50_{-0.07}^{+0.08} \\
\sin ^{2}\left(\theta_{13}\right) & <0.02
\end{aligned}
$$

All errors are at $1 \sigma$ with the exception of the limit for $\theta_{13}$, which is at $90 \%$ C.L. These values were obtained from a global three-flavour analysis to all experimental

\footnotetext{
${ }^{3}$ In two-flavour analyses based on one-mass-scale-dominance, $\Delta m^{2}=\Delta m_{31}^{2}=\Delta m_{32}^{2}$ is used. We normally use $\Delta m_{32}^{2}$ in the text. Here however, a full three-flavour treatment was performed.
} 
data available in June 2006.

The aspects of neutrino oscillations which remain completely unknown are: the sign of $\Delta m_{32}^{2}$ and therefore the mass hierarchy and whether there is CP violation in the leptonic sector. These issues will be addressed by future neutrino oscillation experiments, which will be briefly discussed in the following section.

\section{$2.4 \quad$ Future experiments}

Future neutrino experiments are driven by the "missing piece" in the PMNS matrix, the element $U_{e 3}$, which involves the third mixing angle $\theta_{13}$ and the CP violating phase $\delta$. The most promising techniques to tackle these questions are long-baseline accelerator neutrino experiments and reactor neutrino experiments.

Long-baseline neutrino oscillation experiments in the near future will follow a new approach: off-axis beams. Both the planned $\mathrm{NO} \nu \mathrm{A}[63]$ experiment in the US and the T2K [64] experiment in Japan, which is under construction, make use of this technology. By putting the detectors off-axis from the beam direction (or pointing the beam to miss the detector), the pion decay kinematics result in a narrow-band neutrino energy spectrum.

The T2K (Tokai to Kamioka) experiment will aim neutrinos created in the JPARC accelerator complex in Tokai at the Super-Kamiokande detector, $295 \mathrm{~km}$ away. The neutrino beam will be 2-3 degrees off-axis from the direction of Super$\mathrm{K}$, resulting in a peak neutrino energy of $\sim 0.6 \mathrm{GeV}$. T2K will search for $\nu_{e}$ appearance to detect the sub-dominant $\nu_{\mu} \rightarrow \nu_{e}$ oscillations. It is expected that the sensitivity in a 5 year run will be an order of magnitude better than the CHOOZ limit. If $\nu_{e}$ appearance is observed initially, T2K could run with $\bar{\nu}_{\mu}$ to explore CP violation. Other measurements for T2K include a very precise $\nu_{\mu} \rightarrow \nu_{\tau}$ measurement and a search for sterile neutrinos from the disappearance of neutral current events. 
If approved, $\mathrm{NO} \nu \mathrm{A}$ will use an upgraded NuMI neutrino beam and a new $30 \mathrm{kt}$ liquid scintillator detector located at a new site approximately $810 \mathrm{~km}$ away. $\mathrm{NO} \nu \mathrm{A}$ is expected to have similar sensitivity to $\theta_{13}$ as T2K. Because of its longer baseline compared to $\mathrm{T} 2 \mathrm{~K}, \mathrm{NO} \nu \mathrm{A}$ can use matter effects to determine the sign of $\Delta m_{32}^{2}$ and thus the mass hierarchy.

Long baseline $\nu_{e}$ appearance experiments always measure a combination of $\theta_{13}$ and $\delta$. Another approach is to look for $\bar{\nu}_{e}$ disappearance over short baselines, using nuclear reactors as a neutrino source. This is the goal of the next generation reactor experiment Double Chooz [65]. Double Chooz is essentially a larger version of the CHOOZ experiment, which obtained the current best limit on $\theta_{13}$. Double Chooz will use two detectors at different distances, improving the measurement of the absolute flux. Since it is a disappearance experiment, it has no dependency on the $\mathrm{CP}$ violating phase or the atmospheric mixing angle $\theta_{23}$. It therefore allows an unambiguous determination of $\theta_{13}$. The projected sensitivity for a five-year run is $\sin ^{2}\left(2 \theta_{13}\right)=0.02$.

Taken together, these future experiments should be able to substantially improve our understanding of the remaining unknowns in neutrino physics. And there is of course always the possibility of making exciting new discoveries. 


\section{Chapter 3}

\section{The MINOS experiment}

\subsection{Overview of the experimental layout}

MINOS [66] is a long-baseline neutrino oscillation experiment. It makes use of the NuMI neutrino beam at Fermi National Accelerator Laboratory (Fermilab) near Chicago/USA.

The NuMI beam [67] is measured in two separate locations, as shown in the schematic 3.1. The MINOS Near Detector is located $\sim 1 \mathrm{~km}$ downstream of the neutrino production target. It records neutrino interactions in every beam spill, therefore collecting a very large event sample which allows measurement of the neutrino beam with very high precision. The beam is then sampled a second time by the MINOS Far Detector, $735 \mathrm{~km}$ away from the source in the Soudan Un-

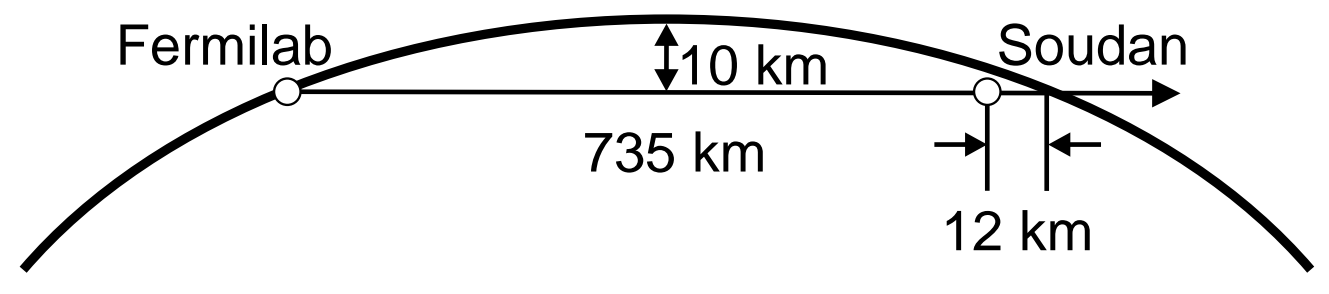

Figure 3.1: Schematic of the MINOS experiment. The two detectors are located at Fermilab and in the Soudan Underground Laboratory, $735 \mathrm{~km}$ from the neutrino source. 


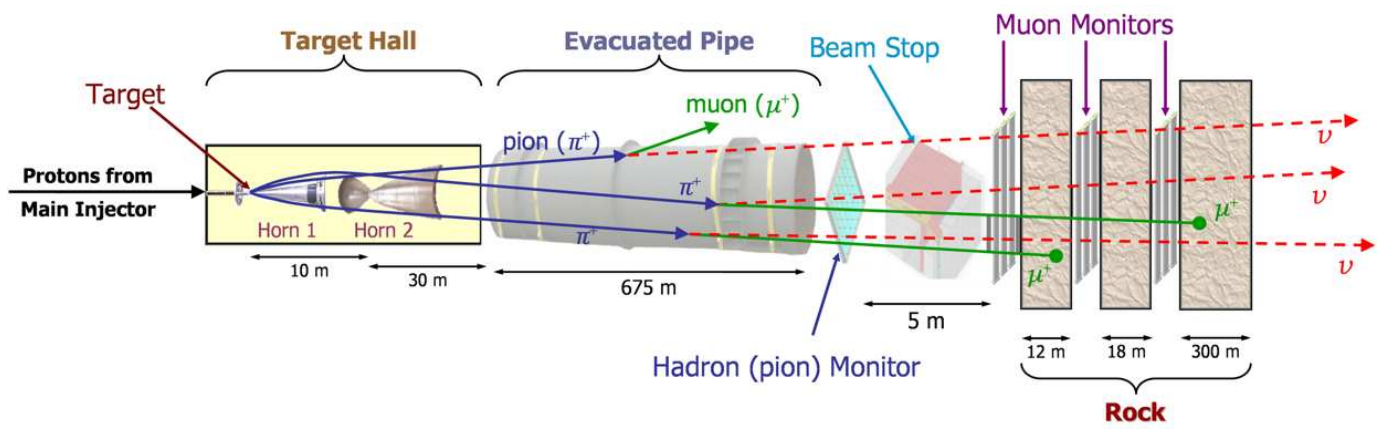

Figure 3.2: The major components of the NuMI beamline. Positively charged mesons produced in the target are focused by two magnetic horns and subsequently decay in a $675 \mathrm{~m}$-long evacuated volume producing predominantly $\nu_{\mu}$. The remaining hadrons are stopped in a beam absorber at the end of the decay pipe.

derground Laboratory in northern Minnesota. By comparing the energy spectra and flavour composition in the two different detectors, MINOS is able to determine neutrino oscillation parameters and distinguish between different theoretical scenarios with high significance.

\subsection{The NuMI beam}

The NuMI beamline at Fermilab is purpose-built to produce a high intensity neutrino beam for MINOS and possible future neutrino experiments such as the offaxis experiment $\mathrm{NO} \nu \mathrm{A}$ [68]. The $120 \mathrm{GeV}$ proton beam extracted from the Main Injector storage ring is allowed to strike a graphite target, producing a spray of secondary particles. Charged mesons from these secondaries are focused by a system of magnetic focusing devices and subsequently decay in an evacuated decay volume, producing neutrinos. The major components of the NuMI beamline are shown in figure 3.2. In the following section, each of these components will be described in some detail. A more complete description of the NuMI beam line can be found in $[67,69]$.

The first step in the creation of the NuMI beam is the acceleration of the 
primary proton beam. This is done in a series of accelerators involving a linear accelerator (Linac) and two circular machines called the Booster and Main Injector. The Linac accelerates the protons from the source to $400 \mathrm{MeV}$. The Booster further accelerates them to $8 \mathrm{GeV}$, the injection energy of the Main Injector. In the Main Injector, the protons are accelerated to their final energy of $120 \mathrm{GeV}$, at which point they are extracted and bent downward at an angle of $58 \mathrm{mrad}$ to direct the beam towards Soudan. The whole acceleration and extraction cycle takes $1.6 \mathrm{~s}$, leading to a minimum repetition rate of $1.9 \mathrm{~s}$. The resulting beam spill contains typically $2.5 \times 10^{13}$ protons and has a duration of $\sim 9.7 \mu \mathrm{s}$ in the case where MINOS receives all 6 batches from the Main Injector. Recently, a peak intensity of $4 \times 10^{20}$ protons per spill has been achieved. NuMI is designed to provide an average beam power of up to $400 \mathrm{~kW}$.

The extracted proton beam is focused and steered onto a segmented, watercooled graphite target, $0.95 \mathrm{~m}$ in length. In order to minimise re-absorption in the target, the target is only $6.4 \mathrm{~mm}$ wide while providing 1.9 hadronic interaction lengths to maximise meson production.

The positively charged mesons created in the target are subsequently focused by a pair of magnetic focusing devices called "horns". The NuMI horns are doubleparabolic magnetic lenses creating a toroidal field with a peak strength of $3 \mathrm{~T}$. They are operated in pulsed mode with a nominal current of $200 \mathrm{kA}$ and cooled by a water spray mechanism [67].

After being focused by the horns, the positively charged pions and kaons enter an evacuated cylindrical volume where they decay to produce a muon and a muonneutrino. This establishes the neutrino beam. The decay pipe is $675 \mathrm{~m}$ long, $2 \mathrm{~m}$ in diameter and evacuated to a residual pressure of approximately 1 torr. Hadrons reaching the end of the decay volume without decaying are stopped in a beam absorber following the decay pipe. The beam absorber consists of a water-cooled aluminium core surrounded by a layer of steel blocks and an outer layer of concrete. 

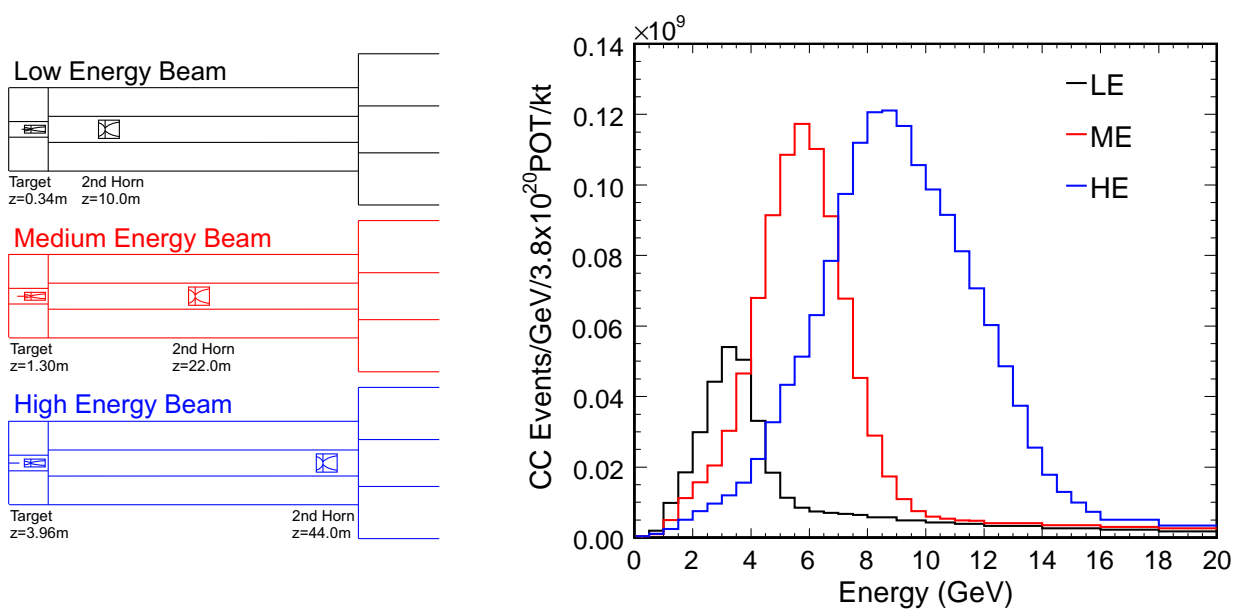

Figure 3.3: Different NuMI beam settings. The NuMI beam spectrum can be varied by moving the target and/or the second focusing horn.

The remaining muons from the meson decays are stopped in the $\sim 300 \mathrm{~m}$ of rock that separate the MINOS Near Detector hall from the beam absorber.

The resulting neutrino beam consists of $97.8 \% \nu_{\mu}$ with small contributions of $\bar{\nu}_{\mu}(1.8 \%)$ and $\nu_{e}(0.4 \%)$ from wrong-sign pions and kaons created in the target as well as $\mu$-decay. It is conceivable that the current in the magnetic horns will be reversed in the future to turn NuMI into an anti-neutrino beam in order to test CPT conservation.

One of the unique features of the NuMI beam line is the fact that the neutrino energy spectrum is tunable. Three of the possible configurations referred to as Low Energy (LE), Medium Energy (ME) and High Energy (HE) Beam, are shown in figure 3.3. The different beam energies are achieved by moving the target and/or second horn with respect to the first horn, similarly to moving lenses in an optical system. For this reason the target is installed in a special carrier which allows the target to be moved longitudinally by up to $2.5 \mathrm{~m}$. This way, pions and kaons of different momenta are selected and focused into the decay volume, resulting in different neutrino energy spectra.

At the time of writing of this thesis, MINOS has mainly taken data in the low 
energy (LE) beam configuration which maximises the sensitivity to neutrino oscillations at the parameter values suggested by the Super-Kamiokande experiment. In addition, smaller data samples have been taken in configurations where the target has been pulled back by $1 \mathrm{~m}$ and $2.5 \mathrm{~m}$ with respect to the LE configuration. These settings are referred to as medium (ME) and high energy (HE) beam. In addition, smaller data samples with lower and higher than nominal horn currents have been recorded.

\subsection{Detector technology}

MINOS is a three detector experiment. The two main detectors, the Near and Far Detectors, sample the NuMI beam at different distances to the neutrino production target. The Near Detector measures the neutrino energy spectrum close to the source while the Far Detector measures the neutrinos after they have been allowed to oscillate, at a distance of $735 \mathrm{~km}$ in the Soudan iron mine in Minnesota, USA. In addition to the two main MINOS detectors a smaller third detector (CalDet) [70] was constructed to measure the detailed responses of the detectors in a charged particle test beam at CERN.

All MINOS detectors are steel and scintillator sampling calorimeters. The Near and Far Detectors are in addition equipped with a magnetic field to allow charge sign identification of muons. They are designed to be functionally as similar as possible while at the same time they have to operate in very different environments.

In the following section we will describe the detector technology which all of the MINOS detectors have in common. The subsequent sections will deal with the specifics of each of the different detectors. 


\subsubsection{Detection principles}

The basic detector unit is a "plane" consisting of a $2.54 \mathrm{~cm}$ thick sheet of steel mounted with $1 \mathrm{~cm}$ of solid polystyrene scintillator followed by an air gap, making up a total plane spacing of $5.95 \mathrm{~cm}$.

The steel provides the bulk of the detector mass for the neutrinos to interact with while the scintillator strips are the active detector element. The passage of charged particles through the detector generates scintillation light which is captured by wavelength shifting fibres and read out by multi-anode photomultiplier tubes (PMTs).

The following sections will describe each of the subsystems of the detectors in turn.

\subsubsection{Steel \& Magnet Coils}

The passive absorber material of the MINOS detectors are $2.54 \mathrm{~cm}$ thick steel plates. In the case of the two main detectors, they are magnetised to allow momentum measurement and charge sign identification of muons and thus distinguish between charged current interactions of neutrinos and anti-neutrinos.

The steel plates are made from low carbon hot rolled steel with an average density of $7.85 \pm 0.03 \mathrm{~g} / \mathrm{cm}^{3}$. The intrinsic radioactivity of each of the $45 \mathrm{manu}-$ facturing runs for the Far Detector was measured to be less than $0.15 \gamma / \mathrm{kg} / \mathrm{sec}$ for $\gamma$ rays above $0.5 \mathrm{MeV}[71]$.

The detectors are magnetised using water-cooled magnetic coils. In the Far Detector, there are two separate 190-turn coils, made from stranded copper wire with Teflon insulation. The Near Detector is equipped with a single eight-turn coil, made from solid aluminium conductors. They provide toroidal magnetic fields with an average field strength of $\sim 1.3 \mathrm{~T}$. 


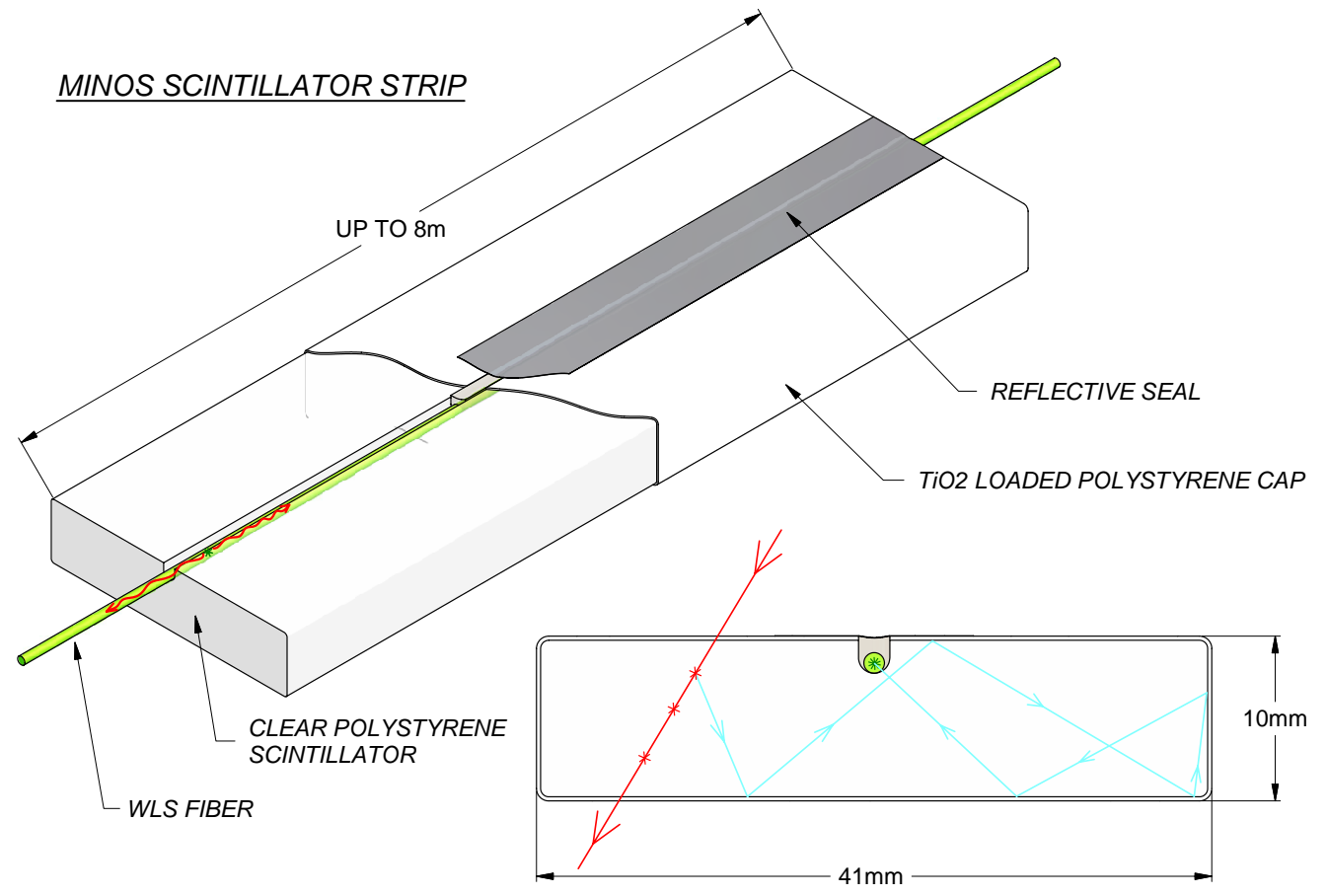

Figure 3.4: Schematic of a single scintillator strip. Light produced by ionising particles is reflected inside the strip by the outer reflective coating. If it is absorbed by the WLS fibre, it is re-emitted isotropically and transported along the fibre to the edges of the detector, where it is routed to the photo-detectors.

\subsubsection{The Scintillator system}

When a neutrino interacts in the absorber material, secondary particles are produced which escape the steel layer and traverse the scintillator layers where they produce scintillation light. The scintillation light is absorbed by wavelengthshifting (WLS) fibres which re-emit and transport the signal to the edge of the detector where it is routed to multi-anode photomultiplier tubes.

The scintillator system is made from $1 \mathrm{~cm}$-thick, solid polystyrene doped with the fluors PPO (2,5-diphenyloxazole) (1\%) and POPOP (1,4-bis(5-phenyloxazol2-yl) benzene) (0.03\%) [72]. It is divided into $4.1 \mathrm{~cm}$-wide and up to $8 \mathrm{~m}$-long co-extruded strips to give spatial resolution. The basic design of the strips is shown schematically in figure 3.4. A WLS fibre is glued into a 2 mm-deep groove which runs along the centre of the "top" side of the strip. The scintillator is sur- 


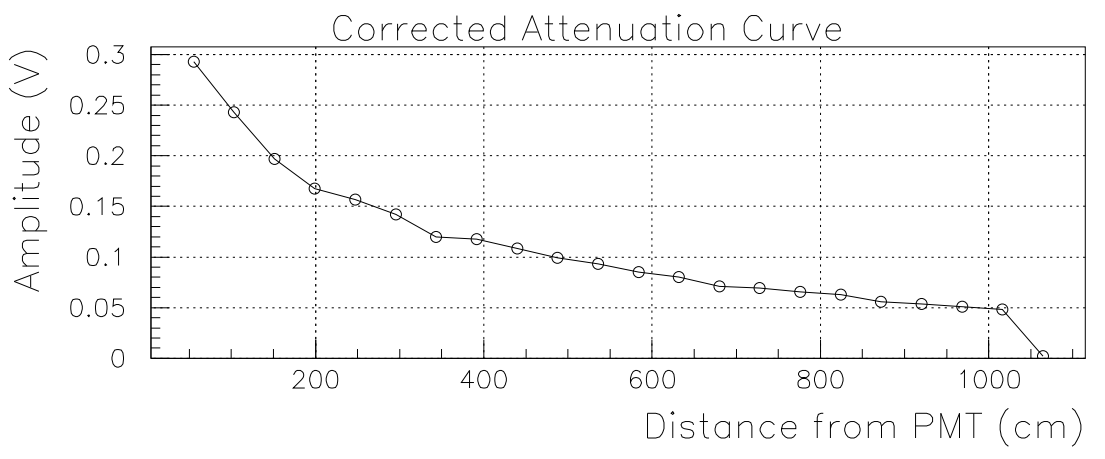

Figure 3.5: Attenuation curve for the wavelength shifting fibre used in MINOS, as measured in a test-stand setup (taken from [73]). The attenuation as a function of distance can be parametrised by a double-exponential decay curve.

rounded by a $0.25 \mathrm{~mm}$-thin layer of titanium-dioxide $\left(\mathrm{TiO}_{2}\right)$-loaded polystyrene which acts as a diffuse reflector which traps the scintillation light until it is eventually absorbed by the WLS fibre.

The WLS fibre is a $1.2 \mathrm{~mm}$ diameter, double-clad polystyrene fibre with $175 \mathrm{ppm}$ of Y11 (K27) fluor produced by Kuraray, Japan. It re-emits the absorbed light isotropically, shifting its wavelength from blue to green (average $\lambda=530 \mathrm{~nm}$ ). Photons emitted along the fibre's central axis are trapped by total internal reflection and therefore transmitted to the edges of the detector. The WLS fibre has very good attenuation properties (see Figure 3.5) which can be parametrised by a double-exponential form with typical constants of $1 \mathrm{~m}$ and $7 \mathrm{~m}$. At the edge of the detector multiple WLS fibres are collected into a "manifold" ending in a single optical connector. From there the light is transported by clear fibre bundles to the photo-detectors.

Consecutive planes of scintillator strips are rotated by $90^{\circ}$ to allow threedimensional tracking. The two different strip orientations are referred to as U-view and V-view respectively. Multiple strips are organised into modules of different size and shape for convenient assembly and in order to cover the steel planes efficiently. Each scintillator module has its own optical connector. The module layout 

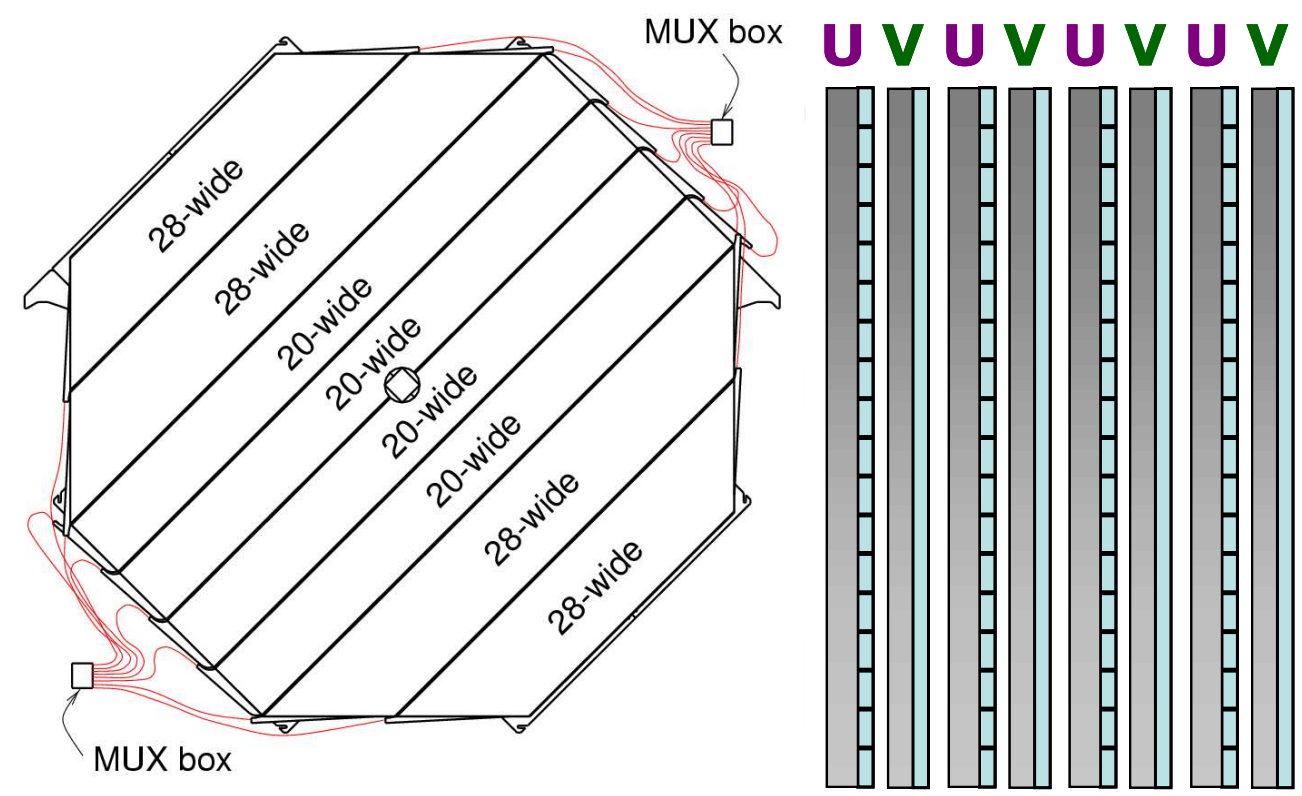

Figure 3.6: Scintillator modules in the Far Detector. The drawing on the right shows the two different strip orientations, referred to as U-view and V-view.

for a Far Detector plane is shown in figure 3.6. A more complete description of the different plane/module geometries used in the different detectors is given in section 3.4 .

\subsubsection{The photo-detectors}

MINOS uses Hamamatsu M16 and M64 multi-anode photomultiplier tubes (PMTs) to convert the light output from the clear fibres to electrical signals which can then be further processed by the data acquisition system. They are housed in steel enclosures which provide light shielding as well as shielding from stray magnetic fields and ambient electronic noise. The small size of the multi-anode PMTs makes them an ideal choice to read out the thousands of scintillator strips in the MINOS detectors and makes them robust to the residual magnetic fields inside their enclosures.

The photo-cathode has a quantum efficiency of $\sim 12 \%$ at $520 \mathrm{~nm}$ and a dark noise rate of $\sim 25 \mathrm{~Hz}$ (M16) and $\sim 4 \mathrm{~Hz}$ (M64) per pixel respectively. Each PMT was 
extensively tested to make sure it meets the MINOS requirements for efficiency, gain, crosstalk and linearity. Further details can be found in $[74,75]$.

Due to the vastly different rates of neutrino interactions, the Near and Far Detectors use completely different readout electronics. They are described in the individual detector sections 3.4.2 and 3.4.3.

\subsection{The different MINOS detectors}

\subsubsection{The Calibration Detector}

The now decommissioned Calibration Detector is a smaller, unmagnetised version of the main MINOS detectors. It consisted of 60 detector planes, each $1 \mathrm{~m}$ square. It was exposed to protons, pions, electrons and muons at the CERN PS test beams in order to measure the energy and topological responses expected in the Near and Far detectors. In addition, the calibration detector acquired data with both Near and Far Detector electronics thus enabling the study of response variations due to the different readout technologies [76].

\subsubsection{The Far Detector}

The MINOS Far Detector is located $735 \mathrm{~km}$ from the neutrino production target in the Soudan Underground Laboratory in Soudan, MN, at a depth of $705 \mathrm{~m}$ or 2070 meters-water-equivalent. It consists of 484 steel/scintillator planes and two steel-only planes in the shape of a regular octagon with a diameter of $8 \mathrm{~m}$, giving it a total mass of 5400 metric tons. The detector planes are grouped into two supermodules of 249 and 237 planes, separated by a gap of $\sim 1.2 \mathrm{~m}$. Each supermodule is individually magnetised by its own magnet coil.

Each detector plane is fully covered by 192 parallel scintillator strips running at an angle of $45^{\circ}$ from the vertical. They are arranged in eight separate modules as 


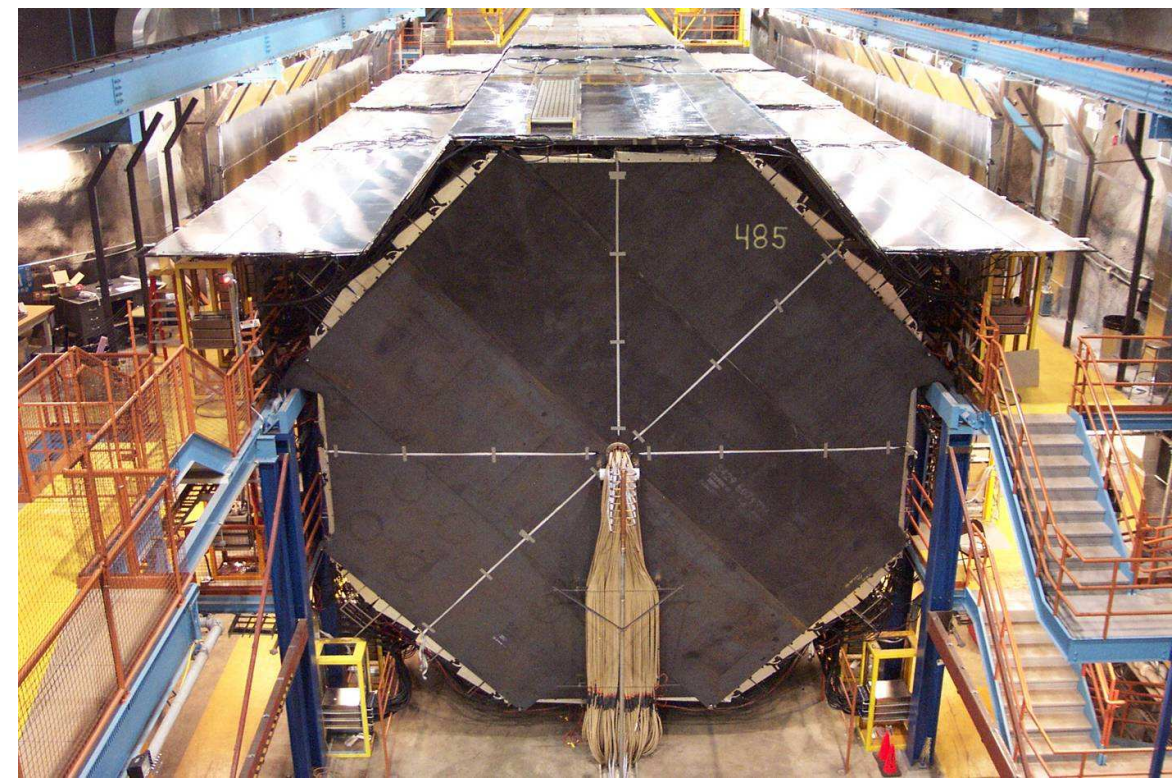

Figure 3.7: A frontal view of the fully installed Far Detector. The cosmic ray veto shield is visible on top of the detector.

shown in Figure 3.6. The strip orientation in consecutive planes is rotated by $90^{\circ}$ to allow three dimensional positioning of the energy depositions in the detector.

The scintillator strips in the Far Detector are read out at both ends using Hamamatsu M16 multi-anode PMTs. To cope with the large number of strips, the fibres from eight different scintillator strips are read out by the same PMT pixel thus reducing the number of electronics channels required by the same factor. This technique is often referred to as multiplexing but should more accurately be called optical summing. The strip-to-pixel patterns are different on the two ends of the scintillator strips. This makes it possible to uniquely identify which strip was actually hit by a particle going through the detector. The complex optical matching needed for this multiplexing is done in the steel boxes housing the PMTs, which are called "MUX boxes". In the Far Detector each MUX box contains three PMTs and reads out one side of two planes $(2 \times 192$ strip ends $)$.

The Far Detector front-end electronics are built around the so-called VA chip, 
a multi-channel $\mathrm{ASIC}^{1}$ produced by Ideas ASA, Norway [77]. The readout system is highly multiplexed and hierarchical, specifically designed to take advantage of the low background rates [78], dominated by spontaneous emission from the WLS fibres and PMT dark noise. The VA chip provides a charge sensitive preamplifier, a shaper and a sample/hold for each channel followed by an analog output multiplexer. One VA chip serves one PMT. Three VA chips are mounted on a common VA Front-end Board (VFB) which is mounted onto the MUX boxes. The digitisation of the signal, pedestal subtraction and sparsification occurs away from the front-end boards on the VA Readout Controllers (VARCs). The VARC provides one analog-to-digital converter (ADC) for six VA chips. The VARCs are housed in VME crates where they are read out by a readout processor which transfers the data to the data acquisition PCs.

The readout of the front-end electronics is triggered by the PMT dynode signal, when one of the signals from the three PMTs connected to a single front-end board surpasses a programmable threshold of nominally $1 / 3$ of a photoelectron (PE). During the course of the work described in this thesis, software which tunes these thresholds for individual front-end boards has been developed and installed in the Far Detector control room. Details are given in Appendix A. Following the dynode trigger, the signals from all 16 PMT pixels are passed to the VARC and digitised. The front-end electronics provide a charge resolution of $2 \mathrm{fC} /$ count and a $1.56 \mathrm{~ns}$ timestamp. After installation of the Far Detector the noise rate from spontaneous light emission in the WLS fibres was found to be higher than expected [79]. In order to cope with this increased rate, an additional coincidence of two dynode triggers within the same VARC is required to trigger the readout. This is referred to as the "2/36 trigger".

In addition to these low-level trigger conditions, the Far Detector readout uses higher-level trigger conditions implemented in software to decide whether the

\footnotetext{
${ }^{1}$ Application-Specific Integrated Circuit
} 
digitised data is saved to file. The main mode of operation during beam data taking is the "spill trigger". It causes the Far Detector to be read out in time with the beam spill. The two MINOS detectors are synchronised using GPS ${ }^{2}$ clocks. When a beam spill occurs, a signal from the beam extraction magnet is sent to the Near Detector where it triggers the readout and is timestamped by the timing system. This timestamp is sent via a TCP/IP connection to the Far Detector where it causes the continuous readout of a $100 \mu$ s window. Another software trigger is the "plane trigger" which activates the readout whenever four out of five contiguous detector planes had a dynode trigger. This trigger condition is used to record atmospheric neutrino interactions which occur outside the spill window. The analysis of atmospheric neutrinos is a secondary goal of the MINOS experiment.

The Far Detector is equipped with a veto shield for cosmic rays positioned above and on the cavern walls to either side of the detector. The veto shield is crucial for the rejection of backgrounds to the analysis of atmospheric neutrinos [80]. The detector plane orientation is optimised for interactions caused by neutrinos coming from Fermilab and it is therefore possible for cosmic rays to enter the detector from the top and come down between the active detector planes. This would imitate a neutrino interaction inside the fiducial region of the detector. The veto shield allows these events to be tagged with high efficiency so they can be removed from the data sample.

\subsubsection{The Near Detector}

The MINOS Near Detector, shown in figure 3.8, is designed to precisely measure the neutrino beam and its interactions. It is located about $1 \mathrm{~km}$ downstream of the NuMI target in a specially constructed cavern $98 \mathrm{~m}$ underground at Fermilab. The Near Detector consists of a total of 282 detector planes, where 153 planes are

\footnotetext{
${ }^{2}$ Global Positioning System
} 


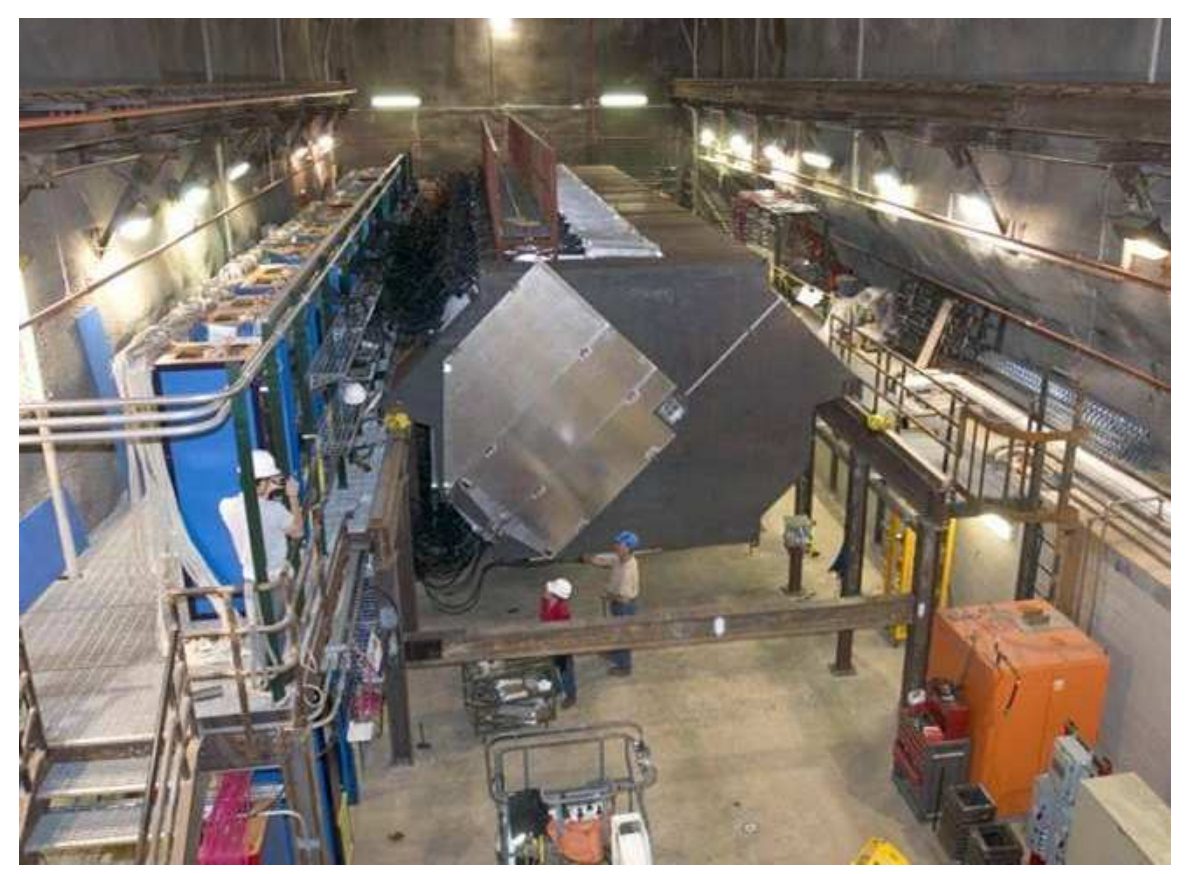

Figure 3.8: The Near Detector during plane installation. The exposed first detector plane is partially instrumented with scintillator modules. The beam centre is located to the left of the coil hole, in the centre of the visible scintillator modules. Electronics racks are visible on the left of the detector.

equipped with scintillator modules and the remainder are steel-only planes, giving it a total mass of 980 tons. It has the shape of a "squashed" octagon measuring $4.8 \mathrm{~m}$ across and $3.8 \mathrm{~m}$ top to bottom. It is magnetised by a single magnet coil.

Due to the very high neutrino flux at the Near Detector location, the detector fiducial volume does not need to be very large in order to record a sufficient number of neutrino interactions. The Near Detector design is therefore driven by a relatively small fiducial region and the need to provide enough steel to achieve the required magnetic field strength of $1.3 \mathrm{~T}$ on average. It is positioned $\sim 1.4 \mathrm{~m}$ off-centre from the neutrino beam so that the coil hole is outside the fiducial region.

The Near Detector is divided into two functionally different parts (see Figure 3.9). The target fiducial volume for the selection of neutrino events is contained in the upstream 120 planes, which are referred to as the calorimeter section. In this section, every plane is instrumented with scintillator in order to provide a precise 


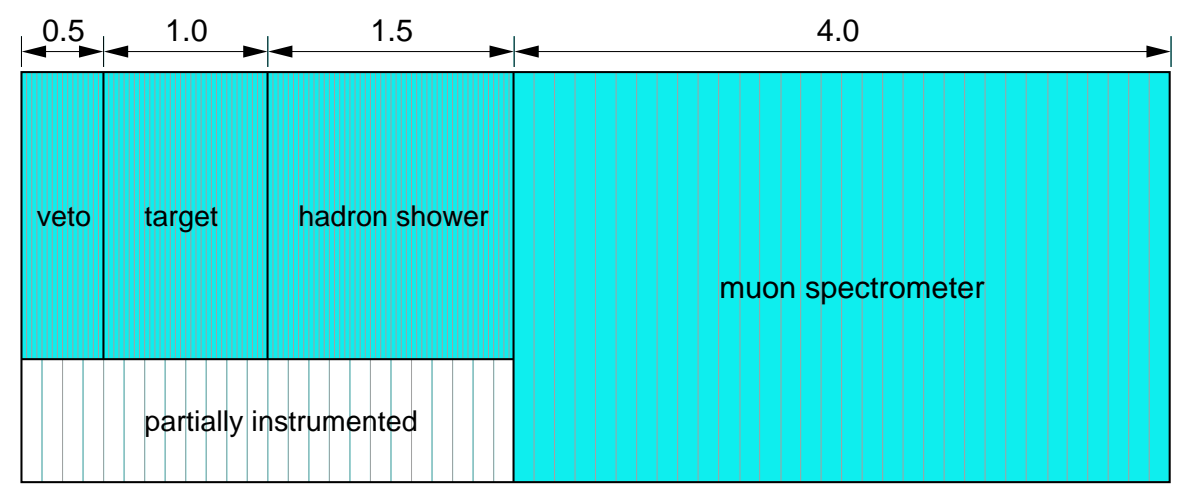

Figure 3.9: The different sections of the Near Detector, as described in the text. The dimensions at the top of the figure reflect the longitudinal extent of steel (in metres) in each section. The veto-, target-, and hadron shower regions form the calorimeter section of the Near Detector.

measurement of shower energy and topology as well as a good determination of the interaction vertex.

The downstream part of the detector is referred to as the spectrometer section. It is used to measure the momenta of high energy muons using the track curvature in the magnetic field or, in case of muons stopping in the detector, their range. In this part of the Near Detector, only one in five planes is instrumented with scintillator strips.

A further difference between the two detector sections is the scintillator coverage of the planes. Figure 3.10 shows the four different plane configurations used in the Near Detector. Every fifth plane in both the calorimeter and spectrometer sections is fully covered with scintillator modules as shown in the bottom two drawings of figure 3.10. The remaining planes in the calorimeter section are partially instrumented, i.e. only a smaller area around the neutrino beam centre is covered with scintillator strips. This is sufficient for precisely measuring the hadronic shower development whereas the fully instrumented planes are used to track outward bending muons.

Similarly to the Far Detector, consecutive planes are rotated by $90^{\circ}$, resulting 

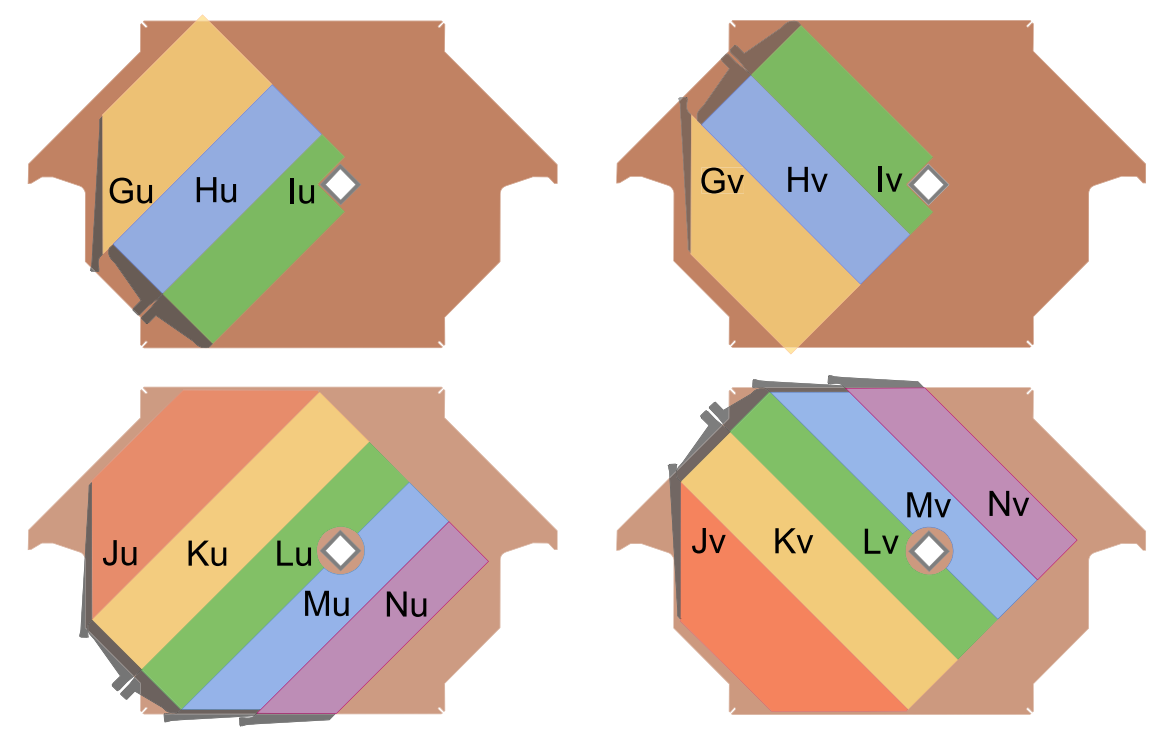

Figure 3.10: The four different plane configurations used in the Near Detector, which differ in the layout of the scintillator modules. The top two figures show the partially instrumented planes in both views, the bottom two figures the fully instrumented planes. The partially instrumented planes are only used in the calorimeter section. The beam is incident midway between the coil hole and the left edge of the plane. 
in two orientations referred to as $\mathrm{U}$ - and $\mathrm{V}$-view respectively.

In contrast to the Far Detector, the scintillator strips in the Near Detector are only read out at one end. Their shorter length of typically $2.8 \mathrm{~m}$ ensures that enough photons created by the passage of a particle reach the photo-cathode of the PMT. The opposite end of each strip is covered with a reflective coating to further increase the light level.

Each strip is read out by a single pixel of a Hamamatsu M64 multi-anode PMT. The partially instrumented planes in the calorimeter section are served by one PMT each, whereas in the fully instrumented planes, one additional PMT is shared by two planes. In order to limit the amount of instrumentation needed, an electrical summing technique is used in the muon spectrometer region of the detector. The signal from four adjacent pixels of the M64 PMTs is summed and read out by a single electronics channel. These adjacent pixels are not connected to adjacent strips, making it possible to resolve the four-fold ambiguity in strip space. The tracking algorithm can however only resolve this ambiguity for tracks starting in the calorimeter section of the detector.

Due to its high rate environment, with multiple neutrino interactions during each beam spill, the Near Detector employs high-speed, deadtime-less front-end electronics based on the QIE chip, originally developed at Fermilab and used in the CDF and KTEV experiments [81]. The QIE chip is a charge-to-current encoder capable of continuous processing for the $\sim 10 \mu$ s duration of a beam spill. It operates at the $53 \mathrm{MHz}$ RF frequency of the Main Injector. The readout charge in each RF bucket is timestamped and digitised separately, resulting in a single digit timing resolution of $\sim 19 \mathrm{~ns}$. The QIE chip sits on a small circuit board (called MENU) together with an ADC and a FIFO for data storage. 16 MENUs are grouped together on a MINDER board which is responsible for the timestamping and which provides power and the control interface to the QIE chips. More details on the Near Detector front-end electronics can be found in [82]. 

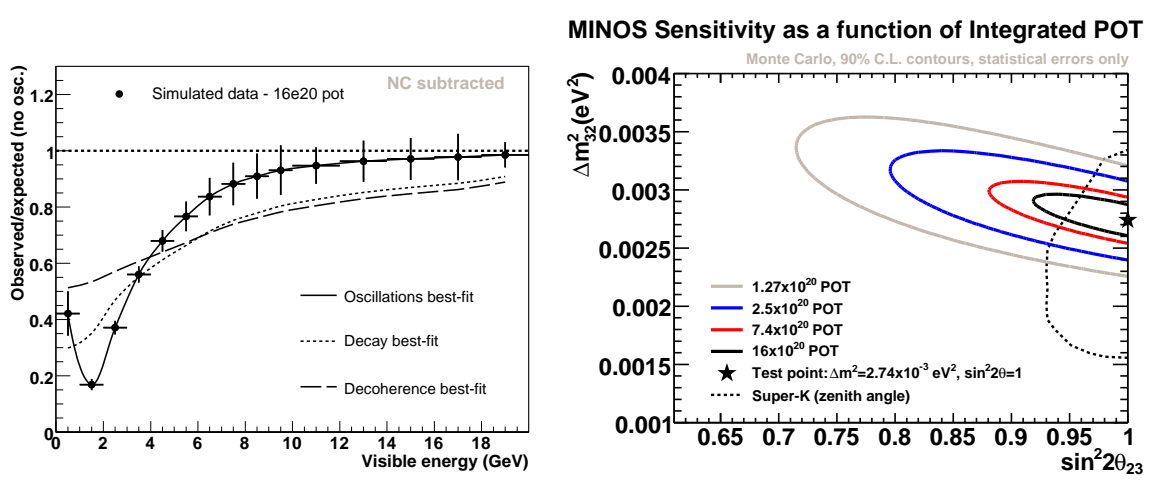

Figure 3.11: Monte Carlo simulation of the MINOS sensitivity. The left plot shows the ratio of simulated data (with oscillations) to an unoscillated Monte Carlo expectation for an exposure of $16 \times 10^{20} \mathrm{POT}$. The lines correspond to the best fit functions for oscillations, neutrino decay [49] and decoherence [50] models. The right plot shows the $90 \%$ C.L. contours for the oscillation parameters $\Delta m_{32}^{2}$ and $\sin ^{2}\left(2 \theta_{23}\right)$ for different exposures, measured in integrated protons-on-target (POT).

\subsection{MINOS physics goals}

MINOS is designed to study neutrino oscillations at the atmospheric mass splitting $\Delta m_{32}^{2}$. By comparing the neutrino energy spectra in the two detectors MINOS will conduct a series of analyses in order to establish that neutrino oscillations are indeed the correct solution to the experimental observations to date and to measure the oscillation parameters with world-leading precision.

The main MINOS measurement is the dominant $\nu_{\mu} \rightarrow \nu_{\tau}$ disappearance channel. The goal is to observe an energy dependent deviation in the number of $\nu_{\mu}$ charged current neutrino interactions in the Far Detector with respect to the prediction from the measured Near Detector flux. By analysing the spectral dependence in detail, MINOS will be able to measure the oscillation parameters $\Delta m_{32}^{2}$ to $\sim 10 \%$ and $\sin ^{2}\left(2 \theta_{23}\right)$ to $\sim 20 \%$ at $90 \%$ C.L. within 2.5 years from the start of beam operations and rule out competing models such as neutrino decay [49] or decoherence [50]. Figure 3.11 shows an oscillation fit to a Monte Carlo experiment as well as the MINOS sensitivity to the oscillation parameters for different exposures. 


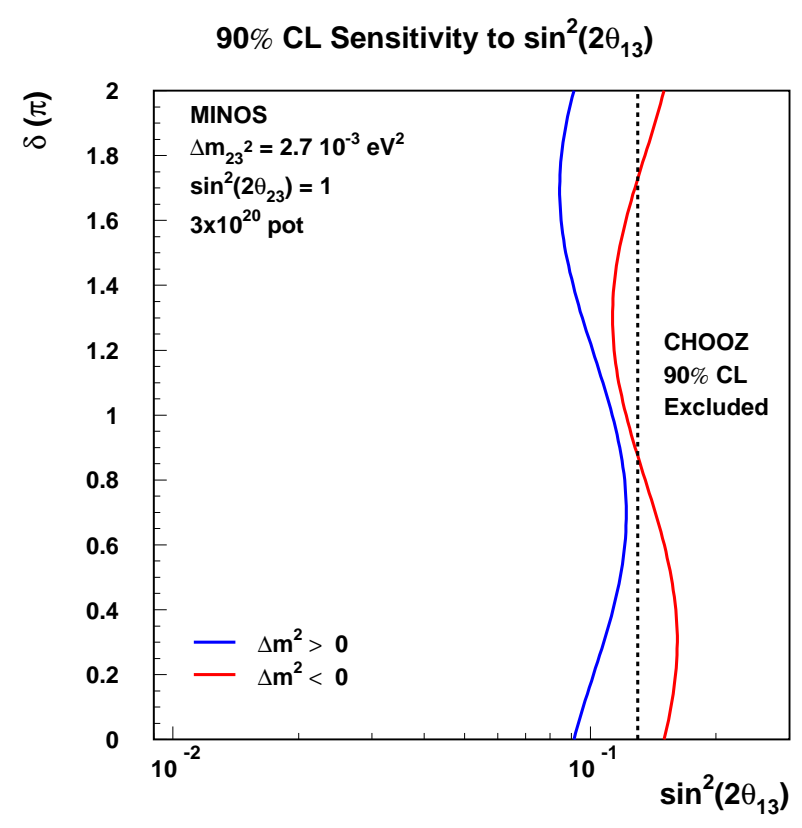

Figure 3.12: MINOS sensitivity to $\theta_{13}$ for $3 \times 10^{20}$ POT. MINOS will have recorded this amount of data by the summer shutdown 2007. Depending on the value of the CP phase $\delta$ and the sign of the mass hierarchy, MINOS has the potential to improve on the current best limit from the CHOOZ experiment.

Other MINOS measurements include the search for sub-dominant $\nu_{\mu} \rightarrow \nu_{e}$ oscillations, oscillations into sterile neutrinos and $\nu_{\mu} \rightarrow \bar{\nu}_{\mu}$ transitions.

$\nu_{\mu} \rightarrow \nu_{e}$ oscillations are driven by the as yet unmeasured angle $\theta_{13}$ in the PMNS mixing matrix for which only an upper limit is known from the CHOOZ reactor experiment [61]. MINOS can measure this parameter by detecting an excess of $\nu_{e}$ charged current neutrino interactions in the Far Detector compared to the number expected from the Near Detector measurement. $\nu_{e}$ events in the Near Detector are due to the intrinsic $\nu_{e}$ component of the NuMI beam from $\mu \rightarrow e+\nu_{e}+\nu_{\mu}$ as well as the less frequent so-called $K_{e 3}$ decay $K \rightarrow \pi^{0}+e+\nu_{e}$. Figure 3.12 shows the MINOS sensitivity to $\theta_{13}$ as a function of the CP phase $\delta$ for $\Delta m_{32}^{2}=2.7 \times 10^{-3}$ and $\sin ^{2}\left(2 \theta_{23}\right)=1$ as calculated from Monte Carlo studies for around 2 years of data taking. With the full data set, MINOS has the potential to either discover $\nu_{\mu} \rightarrow \nu_{e}$ oscillations or improve the CHOOZ limit by a factor of two to three. 

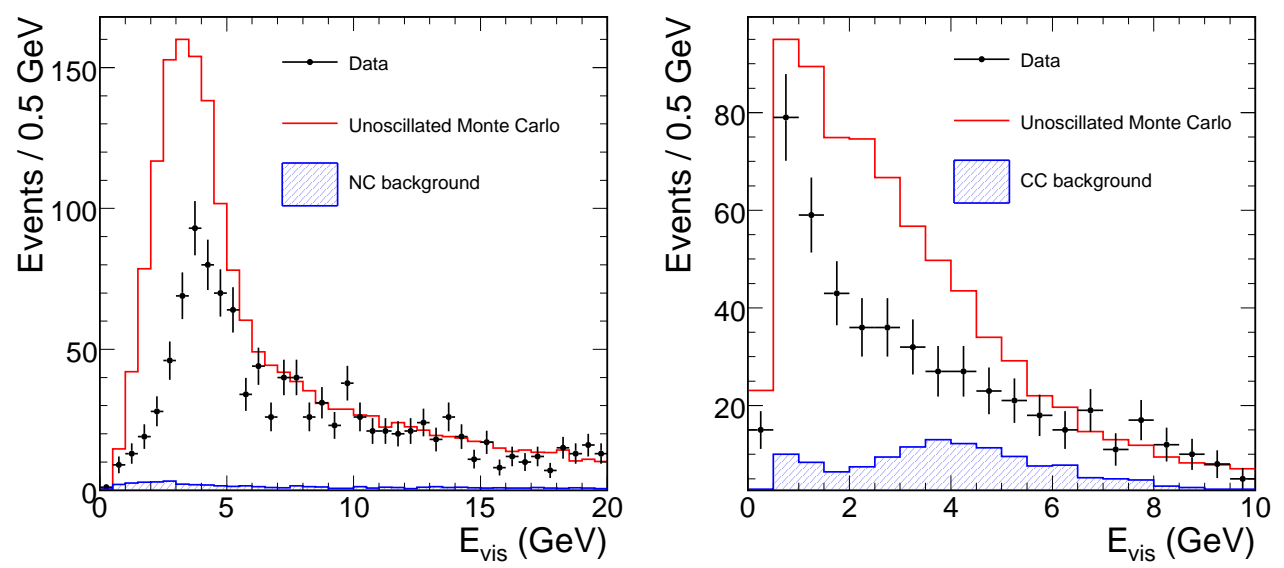

Figure 3.13: Sterile neutrino signal. Oscillations into sterile neutrinos result in a disappearance signal in both the charged current (left) and neutral current (right) energy spectra. This sketch has been obtained from Monte Carlo, assuming $50 \%$ of neutrinos oscillate into a sterile state.

Oscillations into sterile neutrinos could be a way to reconcile the result of the LSND experiment (see Chapter 2) with the accepted interpretation of neutrino oscillations. Sterile neutrinos do not feel any standard model forces apart from gravity. They do however have mass and therefore take part in the propagation of neutrinos through space-time. If neutrino oscillations only involve active neutrinos, neutral current interactions in the Far Detector are unaffected. If however part of the $\nu_{\mu}$ disappearance is due to oscillations into sterile neutrinos, MINOS would observe a deficit in the number of neutral current events. A sketch of a sterile neutrino signal (from Monte Carlo) is shown in Figure 3.13.

\subsection{MINOS data}

In this section, we will briefly describe the different data sets used for the analyses presented here. First, we describe the neutrino beam data recorded at Fermilab's NuMI beam, followed by a short overview of the Monte Carlo simulation programs used in MINOS. 


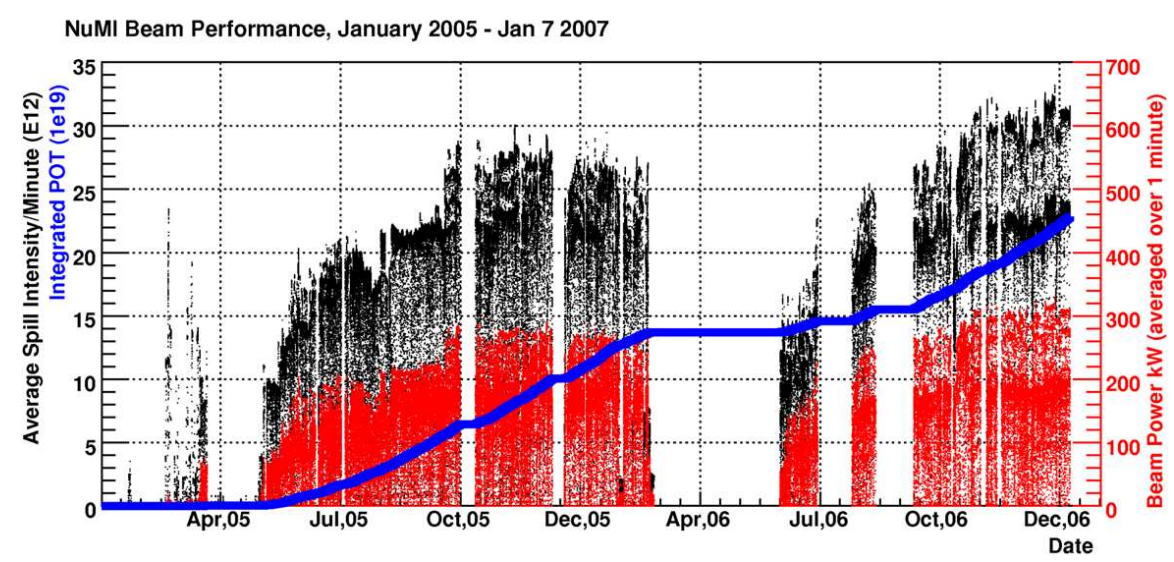

Figure 3.14: Development of beam data acquisition. This figure shows the instantaneous (black) and the integrated (blue) MINOS exposure as a function of time. The beam power in $\mathrm{kW}$ is shown in red. The instantaneous power increases over time as the accelerator performance improves. The first running period ended in February 2006.

\subsubsection{Neutrino beam data}

MINOS recorded the first neutrino interaction from the NuMI beam during a commissioning run in December 2004. Regular data taking started at the end of March 2005. Unless otherwise stated, the data used for the analyses presented here are taken from the first official MINOS run period spanning from March 2005 to February 2006. The total exposure of this run period is $1.27 \times 10^{20}$ protonson-target for the low energy beam configuration. The development of beam data acquisition with time is shown in Figure 3.14.

Smaller data samples have been taken in other beam configurations in order to study hadron production in the NuMI target. This is an important step for the accurate prediction of the neutrino flux in the MINOS detectors. It is described in detail in chapter 7 .

The neutrino beam spectrum was varied by moving the NuMI target with respect to the first horn and by changing the strength of the horn current. These variations were combined to produce six beam configurations, summarised in Table 3.1. 


\begin{tabular}{lcccc}
\hline \hline $\begin{array}{l}\text { Beam } \\
\text { configuration }\end{array}$ & $\begin{array}{c}\text { Target } \\
\text { position }(\mathrm{cm})\end{array}$ & $\begin{array}{c}\text { Horn } \\
\text { current }(\mathrm{kA})\end{array}$ & $\begin{array}{c}\text { Typical } \\
E_{\nu}(\mathrm{GeV})\end{array}$ & $\begin{array}{c}\text { Exposure } \\
\left(10^{18} \mathrm{POT}\right)\end{array}$ \\
\hline \hline LE10/0kA & 10 & 0 & $9.0^{a}$ & 2.69 \\
LE10/170kA & 10 & 170 & 3.0 & 1.34 \\
LE10/185kA & 10 & 185 & 3.0 & $127.0^{b}$ \\
LE10/200kA & 10 & 200 & 3.0 & 1.26 \\
LE100/200kA & 100 & 200 & 5.2 & 1.11 \\
LE250/200kA & 250 & 200 & 10.2 & 1.55 \\
\hline \hline
\end{tabular}

${ }^{a}$ The horn-off beam is unfocused and has a very broad energy distribution.

${ }^{b}$ This is the standard low-energy beam configuration.

Table 3.1: Different beam configurations recorded over the run period, obtained by moving the target with respect to the horns and by varying the horn current. These different data samples were used to study hadron production in the NuMI target and to accurately predict the neutrino flux seen by the MINOS detectors (see chapter 7 for details).

\subsubsection{Monte Carlo data}

In order to evaluate the sensitivity of an experiment for a particular measurement, investigate systematic uncertainties and compare the measured data to different theoretical models, MINOS makes use of simulated data. Like most particle physics experiments, MINOS uses a Monte Carlo technique for these simulations.

Generating Monte Carlo data for MINOS is a complex task. It involves creating a computer model of every part of the experiment, from the NuMI target and the decay volume to the two detectors. The hadron production on the NuMI target, their decays to produce neutrinos as well as the interaction of the neutrinos in the detectors, the energy deposition of the final state particles and their conversion into light and subsequent electronic signals have to be simulated. The output of the simulation is most conveniently written in files with an identical format to the real data which can subsequently be passed to the event reconstruction algorithms. In the following section, the different components of this Monte Carlo simulation will be discussed briefly. 
The simulation of the NuMI neutrino beam line is done using the GEANT-3 [83] based program GNUMI. It includes the simulation of all relevant components in the beam line as well as the pion and kaon production in the target resulting in the neutrino beam measured by the MINOS detectors. The simulation of the hadron production is done in FLUKA, a general purpose tool for simulating particle transport and particle interactions in matter, developed by CERN and INFN Milano [84, 85].

The neutrino-nucleus interactions in the MINOS detectors are simulated using NEUGEN [86], a FORTRAN based neutrino event generator originally written for the Soudan 2 experiment. It uses a pre-tabulated library of neutrino cross-sections to improve execution speeds. NEUGEN will eventually be replaced by a new event generator called GENIE [87], which is a fully object-oriented $\mathrm{C}++$ development.

The simulation of the MINOS detectors is achieved in a GEANT-3 based framework called GMINOS. In addition to implementing the detector geometries, GMINOS interfaces with the NEUGEN event generator and passes events to GEANT-3 for tracking and recording detector truth hits. In order to simulate the hadronic interactions of final state particles delivered by NEUGEN, GMINOS uses the GCALOR package [88]. GCALOR is a Monte Carlo package developed at Oak Ridge National Laboratory for the design and analysis of calorimeter systems.

The final step in the simulation chain is the transformation of energy depositions into light signals and their conversion into electric signal in the PMTs and front-end electronics. This step is achieved by the two software packages Photon Transport and Detsim. Both packages are written in $\mathrm{C}++$ and use the ROOT [89] analysis framework. Photon Transport simulates the conversion of energy depositions into light, their transport to the PMT photocathodes and the creation of the conversion to photo-electrons. Detsim simulates the amplification of the photoelectron signal in the PMT dynode stages, the signal path through the front-end electronics and finally the data acquisition. 


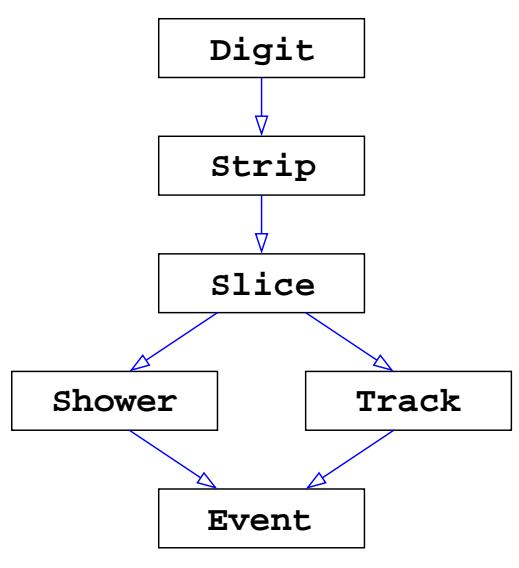

Figure 3.15: MINOS event reconstruction. This figure shows a step-by-step schematic of the MINOS event reconstruction chain, starting with individual digits and ending with events.

The end product of the complete Monte Carlo simulation chain is an object named RawDigitDataBlock which is processed by the event reconstruction algorithms identically to real data.

\subsection{MINOS event reconstruction}

This section gives a brief summary of the basic building blocks of the event reconstruction in MINOS. It does not describe any of the algorithms in use, but defines some terms which are commonly used throughout this thesis. A flowchart of the output of the main steps in the reconstruction chain is shown in Figure 3.15. As the reconstruction proceeds, the low level hits from the detector are transformed into complete, separate neutrino interactions via the following entities.

Digit: The smallest unit of data recorded in the detector (both in real data and Monte Carlo) is referred to as a digit. A digit records the charge in ADC counts, time in TDC counts and location in the detector of a single signal digitisation. 
Strip: Multiple digits in the same physical scintillator strip occurring within a time window are combined into a strip. The digits used to form a strip are meant to originate from the same energy deposition. In the Far Detector, the pulse from each end of the scintillator strip forms a separate digit. In the Near Detector, there are multiple digits for each signal pulse, corresponding to the $\sim 19 \mathrm{~ns} \mathrm{RF}$ buckets. A strip is the smallest unit used in the event topology variables used in this document.

Slice: A group of strips which are close in both space and time are combined to form a slice. This step is crucial in the Near Detector, where multiple neutrino interactions occur during each beam spill. The strips in a slice are meant to originate from a single neutrino interaction. Because of the large event rate in the Near Detector, this is not always the case. In the Far Detector, most beam spills only contain one slice.

Shower: Strips in a slice which are clustered in space are combined into a shower. Showers can be caused by the neutrino interaction with a nucleus, electrons from $\nu_{e}$ charged current interactions or delta rays from a muon track. The shower reconstruction algorithm also determines a vertex for each shower, using topology and timing information.

Track: The reconstruction of tracks from the strip information in a slice proceeds in two steps: track finding and track fitting. First, small track-like clusters are found and, if possible, joined up to form the full track. Then, the thus found track is fitted using a Kalman filtering procedure. The track fit provides the charge sign of the track and the momentum from curvature in the magnetic field of the detector. 
Event: In this final reconstruction step, showers and tracks are combined to form events. This step takes into account the spatial separation of tracks and showers within the slice. The reconstructed event represents the best estimate of the energy depositions caused by a single neutrino interaction. For example, a typical $\nu_{\mu}$ charged current event consists of a reconstructed track from the muon and a reconstructed shower from the struck nucleus.

The reconstruction also calculates an event vertex, based on timing and event topology. The vertex represents the best estimate of the neutrino interaction point. Tracks and showers have vertices of their own. The event vertex can but does not have to coincide with the track and shower vertices. 


\section{Chapter 4}

\section{Preliminary sensitivity study: The 2005 Mock Data Challenge}

The 2005 Mock Data Challenge (MDC) was an early data analysis exercise in MINOS exclusively using Monte Carlo simulated data. It took place before the NuMI beam first turned on and it gave the collaboration and its analysis working groups the opportunity to exercise and improve the reconstruction algorithms, data handling procedures and the analysis frameworks. It incorporated the best knowledge at the time into the simulations and the reconstruction algorithms. Finally, the analysis groups were presented a challenge to actually extract the hidden physics parameters used to generate the mock data.

\subsection{Overview of the NC analysis procedure}

The physics goal of the neutral current analysis is to measure the fraction of neutrinos which oscillate into sterile neutrinos. In the case of pure $\nu_{\mu} \rightarrow \nu_{\tau}$ oscillations, a deficit would be observed in the charged current energy spectrum, whereas the neutral current spectrum would be unaffected. If however a fraction of the $\nu_{\mu}$ oscillate into a sterile state, an additional deficit in the neutral current 
spectrum would be observed.

After separating the neutrino events into charged current and neutral current interactions (see section 4.2), the analysis proceeds in three steps:

\section{Verify Data/MC agreement in the Near Detector}

The large number of neutrino interactions in the Near Detector allows a detailed data/Monte Carlo comparison. The Monte Carlo simulation is improved by fitting changes to the neutrino cross-section parameters to the measured Near Detector data.

2. Apply changes to Far Detector Monte Carlo

If substantial differences between data and Monte Carlo are observed in the Near Detector, the results of the Near Detector cross-section fit are applied to the Far Detector Monte Carlo prediction.

\section{Far Detector oscillation fit}

After the Far Detector Monte Carlo simulation has been thus improved, the measured Far Detector data is fitted for oscillations. The selected charged current and neutral current spectra are fitted simultaneously for the oscillation parameters $\Delta m_{32}^{2}, \sin ^{2}\left(2 \theta_{23}\right)$ and the sterile fraction $f$.

\subsection{Event selection}

The main difference between charged current and neutral current neutrino interactions is the presence (or lack) of a muon in the final state. Charged current events contain a muon track whereas neutral current events consist solely of hadronic activity. Real life is of course somewhat more difficult than this. Tracks in the event can be caused by other particles such as protons or pions or the muon track can be completely obscured by the hadronic shower. Effects like this cause charged current events to be misidentified as neutral currents or vice versa. 
In order to select neutral current neutrino interactions, a set of identical cuts was used in both the Near and Far Detector. In addition to looking for a track in the event the cuts are designed to judge whether a track is a "good" muon track. The cuts also use the event length since neutral current events are generally shorter because there is no muon in the final state. Finally, fiducial cuts are applied to make sure the interaction really occurred inside the detector volume and that the hadronic shower is properly contained in the detector.

\subsubsection{Fiducial cuts}

For this first analysis a simple cylindrical fiducial volume was used. In the Near Detector, the reconstructed vertex of the event has to satisfy the following conditions:

$$
\begin{array}{r}
1 \mathrm{~m}<z<6.2 \mathrm{~m} \\
(x-1.44)^{2}+y^{2}<(1 \mathrm{~m})^{2}
\end{array}
$$

The longitudinal cut ensures that the interaction took place in the calorimeter section of the Near Detector and that the hadronic shower is mostly contained in the calorimeter section as well. Ideally, only the muon track penetrates into the more sparsely instrumented spectrometer region. The transverse cut makes sure that the interaction vertex and most of the shower is contained inside the fully instrumented part of the calorimeter.

In the Far Detector the fiducial volume consists of two parts reflecting the two supermodules which make up the detector. The fiducial cuts are:

$$
\begin{gathered}
1 \mathrm{~m}<z<14 \mathrm{~m} \quad \text { or } 17 \mathrm{~m}<z<28 \mathrm{~m} \\
x^{2}+y^{2}<(3.5 \mathrm{~m})^{2}
\end{gathered}
$$



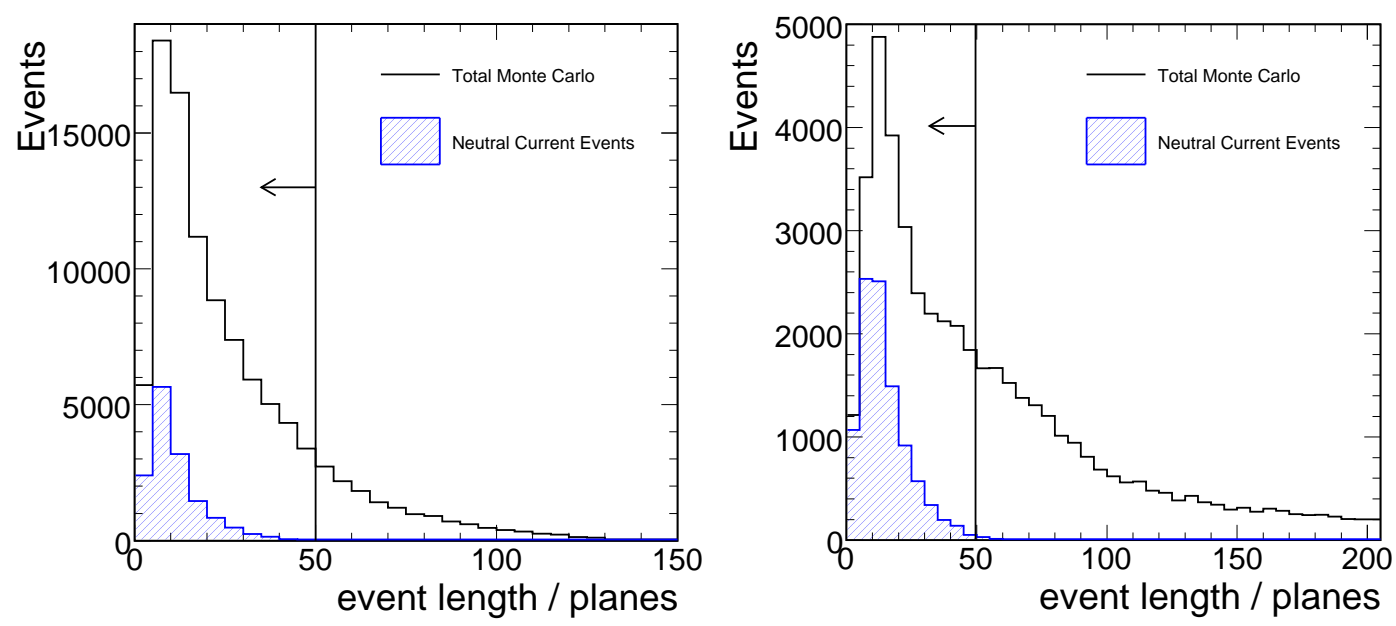

Figure 4.1: Event length $L$ distributions for Near (left) and Far (right) Detector Monte Carlo. The blue hatched distributions show neutral current events. A selection cut is made at 50 planes. The arrow indicates the events which are kept.

Again, they ensure that the interaction occurred far enough away from all edges of the detector.

It is important to point out that for this first analysis, the fiducial cuts were not fully optimised. Hindsight has shown that they were rather aggressive in demanding containment with a considerable loss of efficiency in the Far Detector. In the Near Detector on the other hand, they came too close to the edge of the fully instrumented region. A more optimised set of fiducial cuts for the Near Detector is discussed in Section 5.1.

\subsubsection{Selection Cuts}

The most powerful separator between neutral and charged current events apart from the presence of tracks is the event length $L$ as shown in Figure 4.1 for Monte Carlo. Neutral current events are preferentially short. A selection cut is therefore made at 50 planes. For events without a reconstructed track, this is the only selection cut applied.

For events with tracks, there are two more selection cuts. The first one makes 

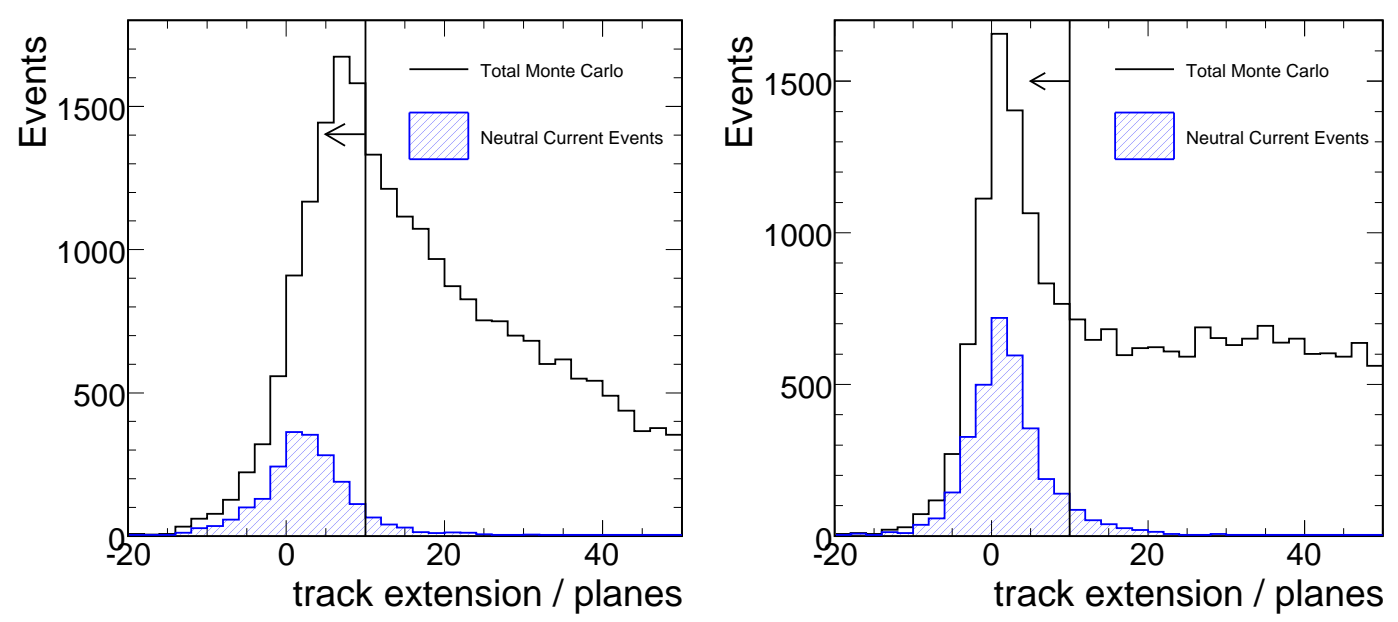

Figure 4.2: Track extension $\epsilon$ distributions for Near (left) and Far (right) Detector Monte Carlo for events with a reconstructed track. The blue hatched distributions show neutral current events. A selection cut is made at +10 planes.

use of the fact that tracks which are found inside the shower and don't "stick out" are less likely to be due to a real muon. A cut is therefore placed on the variable track extension $\epsilon$ which is defined as follows:

$$
\epsilon=p_{t r k}-p_{s h w}
$$

where $p_{\text {trk }}$ and $p_{\text {shw }}$ represent the number of planes crossed by the track and shower respectively. The distributions for this variable are shown in Figure 4.2. A cut is made at a value of +10 in both detectors.

Finally, since neutral current events do not have a real muon track, the fit to the reconstructed track is generally expected to be poorer for neutral current events. To take advantage of this, a cut on the relative fit error of the track $\hat{\sigma}=\frac{\sigma(q / p)}{q / p}$ is applied as shown in Figure 4.3. $q$ and $p$ represent the reconstructed charge and momentum of the track. It should be noted that the track fit will not be bad in all neutral current events since a proton or pion can cause a real track in the detector. This cut will however take advantage of cases where a track is 

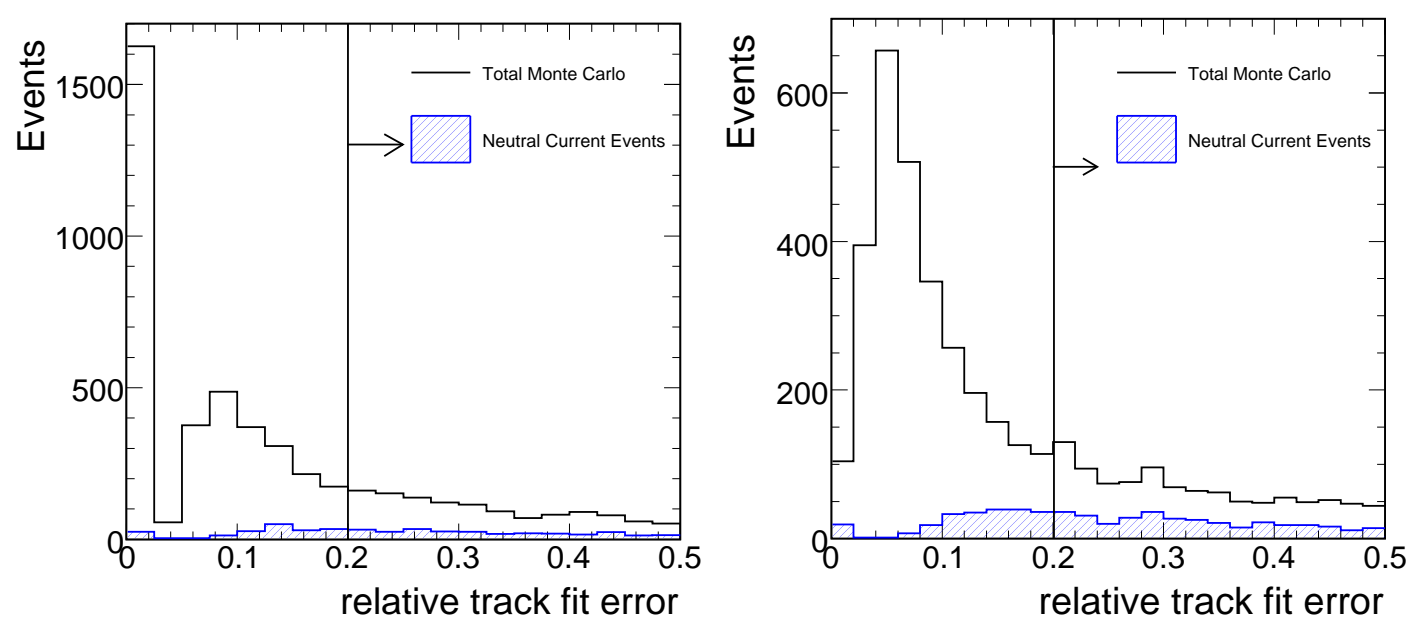

Figure 4.3: Relative track fit error $\hat{\sigma}$ distributions for Near (left) and Far (right) Detector Monte Carlo for events with a reconstructed track. The blue hatched distributions show neutral current events. A selection cut is made at 0.2.

constructed from random hits in the shower.

In summary, an event has to pass the following three cuts in order to be classified as a neutral current event:

- $L<50$ planes

- $\epsilon<10$ planes

- $\hat{\sigma}>0.2$

Events inside the fiducial volume which fail any of the cuts above are classified as charged current interactions.

The efficiencies and purities for this simple event selection as a function of energy are shown in Figure 4.4. Efficiencies and purities are defined for the three selection cuts with respect to the events in the fiducial volume. This means, if all neutral current events reconstructed in the fiducial volume were selected, the selection efficiency would be $100 \%$. Purity is the percentage of correctly selected events in the selected sample. Across the whole energy range the selection efficiency for this selection is $82 \%$ with a purity of $54 \%$ for the Near Detector. For the Far detector, the numbers are: $85 \%$ efficiency and $57 \%$ purity. The statistical 

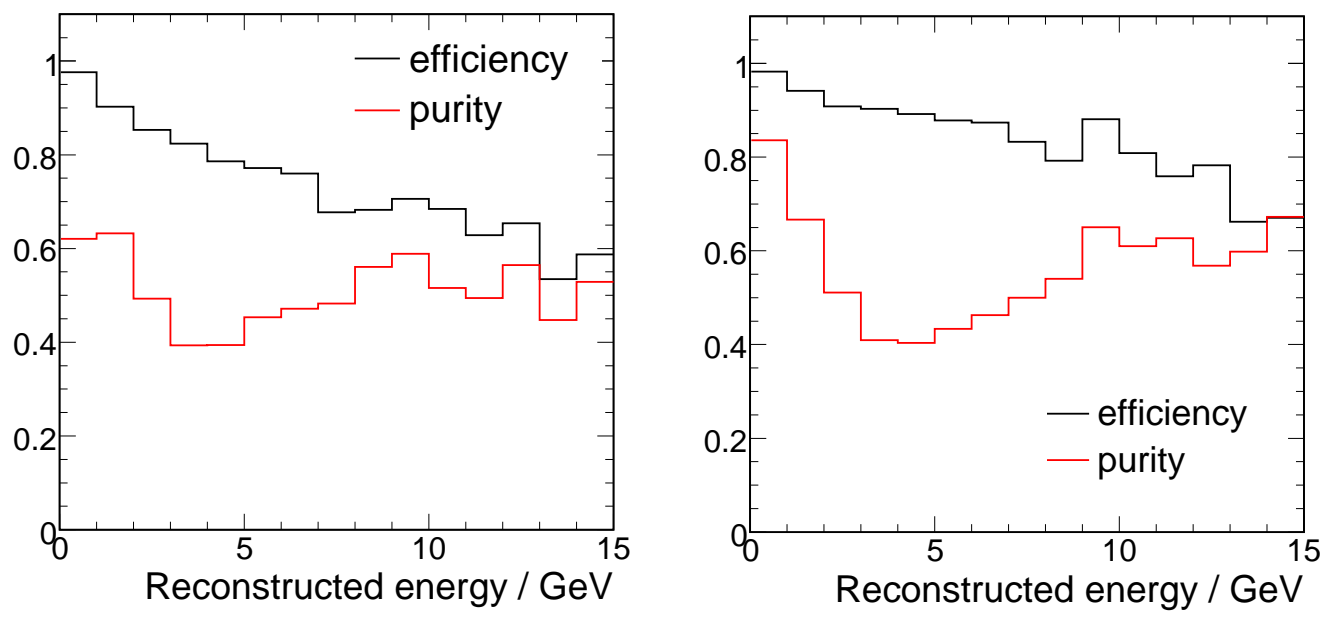

Figure 4.4: Selection efficiency and purity distributions for Near (left) and Far (right) Detector. The general shape of the distributions is very similar in the two detectors. The overall performance of the selection is slightly better in the Far Detector. This is understood in terms of the different reconstruction environments.

errors on these numbers due to Monte Carlo statistics are $\sim 0.9 \%$ (ND) and $\sim 1.5 \%$ $(\mathrm{FD})$.

The difference is understood to come from the very different environments in which the reconstruction algorithms have to perform. An event in the Far Detector is usually simple to pick out, whereas in the Near Detector, several events occur during the same beam spill and need to be separated. This leads to a reduction in reconstruction "quality" and ultimately in a loss in selection efficiency and purity.

\subsection{Near detector fit}

The selected neutrino events in the Near Detector are used to improve our knowledge of neutrino interaction cross-sections and beam flux by means of fitting the observed neutrino energy spectrum. The results of this fit are then used to modify the Monte Carlo simulation to best reflect the measured Near Detector data. Finally, the thus improved Monte Carlo is used in a Far Detector oscillation fit.

In the Mock Data Challenge 2005, the Monte Carlo used to generate the "data" 
samples was modified in three different model parameters, called $M_{A, q e}, M_{A, \text { res }}$ and $K N O_{1 j k}$. The first two are mass parameters in the axial form factor $F_{A}$ for quasi-elastic scattering and resonance production respectively. $K N O_{1 j k}$ are a set of scale parameters linking the neutrino cross-section in the resonance region to exclusive cross-sections at higher energies. The index 1 stands for $C C$ interactions and $j k$ stand for different target nucleons and final state multiplicities. The KNO model is a phenomenological model for hadronization, based on the observation that the scaled multiplicity distribution is independent of $W$.

For the MDC the KNO parameters were only scaled as a whole making them effectively one parameter. More information about these parameters and the $N E U$ GEN event generator as a whole can be found in [86]. The determination of these model parameters, together with the oscillation parameters, forms the challenge posed to the analysis groups.

In order to find the best fit for these parameters, a technique called Monte Carlo re-weighting was applied. When a Monte Carlo model parameter is changed, each event is assigned a new weight depending on the changed parameter and the event's neutrino flavour, target nucleus and event kinematics variables. This way, the effect of a change in Monte Carlo parameters on the neutrino energy spectrum can be evaluated without repeating the whole Monte Carlo simulation and event reconstruction. One downside of this approach is that this procedure only reweights whole events. No new track or shower reconstruction, which could be influenced by the model changes, is performed. These effects on the reconstruction are expected to be small however. Furthermore, it is simply impossible to perform full Monte Carlo simulations for all potential parameter changes with the available computing resources.

The best values for the three modified model parameters are determined using a binned, extended maximum-likelihood fit of the following form: 
Likelihood $\mathcal{L}=\frac{e^{-N} N^{D}}{D !} \prod_{i}\left(\frac{n_{i}}{N}\right)^{d_{i}} \quad$ with $\quad\left\{\begin{array}{l}d_{i}:=\text { data events in bin } i \\ n_{i}:=\text { MC events in bin } i \\ D:=\sum_{i} d_{i}, N:=\sum_{i} n_{i}\end{array}\right.$

The first term is a normalisation term which gives the probability of observing $D$ events in total given a Poisson distribution with mean $N$. The product multiplies the probabilities for observing $d_{i}$ events in bin $i$ given a Monte Carlo expectation of $n_{i}$. The parameters which maximise this likelihood are found using the function minimisation package MINUIT [90]. In order to better constrain the model parameters, both the selected CC and NC spectra are fitted simultaneously.

The result of the fit to the energy spectrum is shown in Figure 4.5. The fit results for the three model parameters are:

- $M_{A, \text { res }}=1.02 \pm 0.02$

- $M_{A, q e}=0.95 \pm 0.03$

- $K N O_{1 j k}=1.01 \pm 0.02$

The results are given in terms of scale factors, i.e. a value of 1 is equivalent to using the default value for this Monte Carlo parameter. The fit results are very close to the nominal values; in fact two of the three parameters are equal to unity within errors. It was therefore decided to use the nominal Monte Carlo for the oscillation fit described in the next section. This was done for simplicity since the fit results from the Near Detector fit are too close to unity to have a significant impact on the final result.

\subsection{Neutrino oscillation fit}

The final step in the analysis chain is to perform an oscillation fit to the Far Detector data and extract the oscillation parameters. 

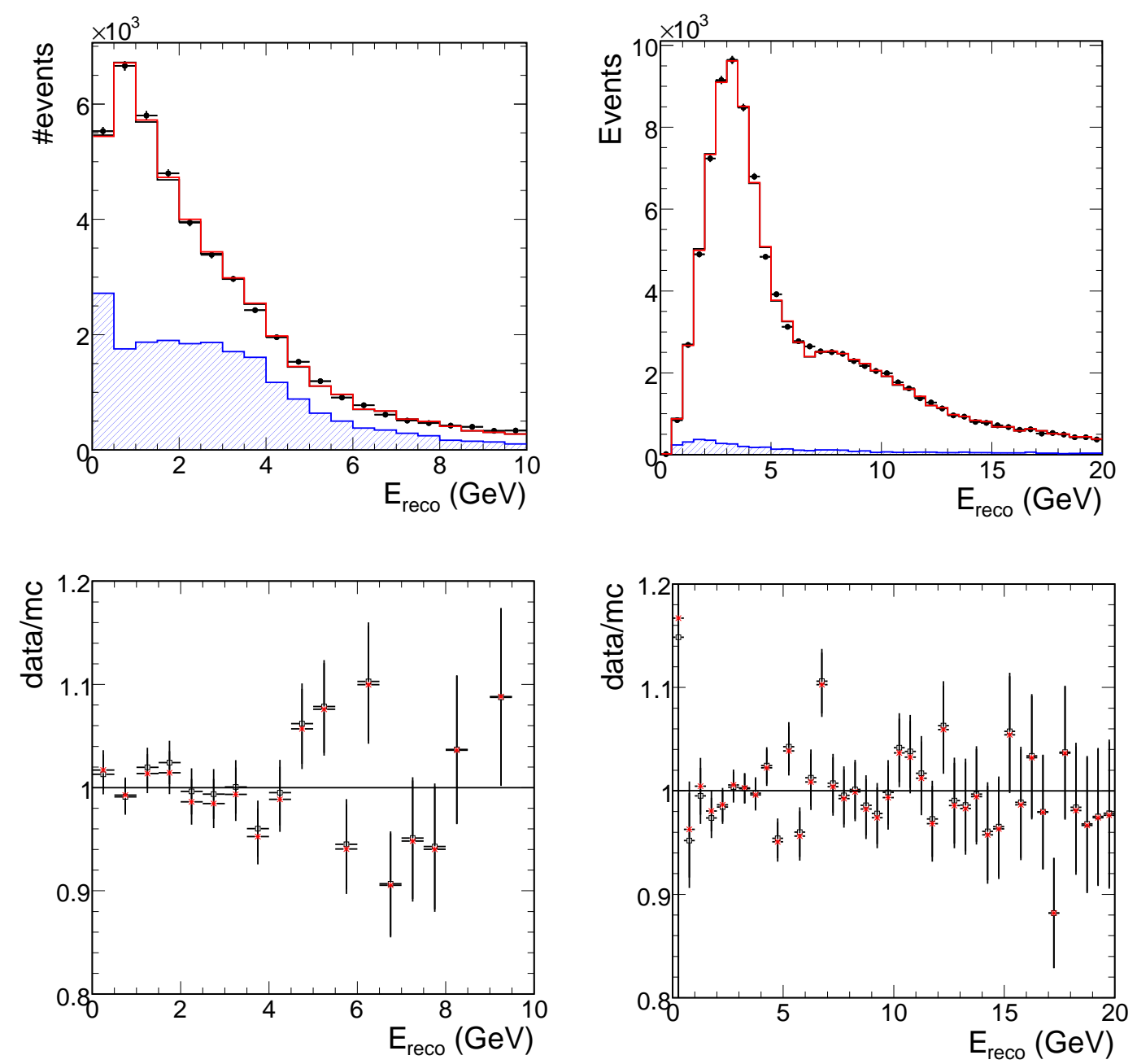

Figure 4.5: The top two figures show neutral current (left) and charged current (right) energy spectra in the Near Detector. The blue, hatched component shows the wrongly selected background. The mock data points are shown in black, the best fit Monte Carlo is shown in red. It is extremely similar to the unfitted Monte Carlo shown as a black line which is mostly obscured by the red line. The bottom two figures show the data/MC ratio before (black) and after the fit (red). 
The Far Detector mock data set corresponds to an exposure of $7.4 \times 10^{20}$ POT which is roughly equivalent to four years of data taking. In the neutral current analysis working group, a fit to a model including oscillations to sterile neutrinos was performed. In this model, the disappearance of $\nu_{\mu}$ is due to oscillations into $\nu_{\tau}$ and $\nu_{s}$. The measured charged current spectrum shows the same deficit as in a normal three-flavour oscillation scenario. The neutral current spectrum however is unaffected by the flavour of the active neutrinos. It is therefore directly affected by the oscillations to sterile neutrinos and will only show a deficit in the case of a non-zero sterile component.

The survival probability for the neutrinos can still be calculated in a twoflavour approximation. In such a model, the $\nu_{\mu}$ are oscillating to a state $\nu_{x}$ which is a superposition of $\nu_{\tau}$ and $\nu_{s}$ :

$$
\left|\nu_{x}\right\rangle=\sqrt{f_{s}}\left|\nu_{s}\right\rangle+\sqrt{\left(1-f_{s}\right)}\left|\nu_{\tau}\right\rangle
$$

The survival probabilities then look like this:

$$
\begin{array}{cr}
P\left(\nu_{\mu} \rightarrow \nu_{\mu}\right)=1-\sin ^{2}\left(2 \theta_{23}\right) \sin ^{2}\left(\frac{\Delta m_{32}^{2} L}{E}\right) & \nu_{\mu} \\
P\left(\nu_{a} \rightarrow \nu_{a}\right)=1-f_{s} \sin ^{2}\left(2 \theta_{23}\right) \sin ^{2}\left(\frac{\Delta m_{32}^{2} L}{E}\right) & \text { active neutrinos }
\end{array}
$$

The first expression shows the survival probability for $\nu_{\mu}$, which drives the observed charged current neutrino spectrum. The second equation shows the survival probability for all active neutrinos. This is relevant for the observed neutral current neutrino spectrum. The additional parameter $f_{s}$ is called the "sterile fraction". It can be interpreted as the fraction of disappeared neutrinos which oscillated into sterile neutrinos.

The Far Detector oscillation fit proceeds in a very similar fashion to the Near Detector fit, detailed in Section 4.3. The Far detector Monte Carlo simulation 

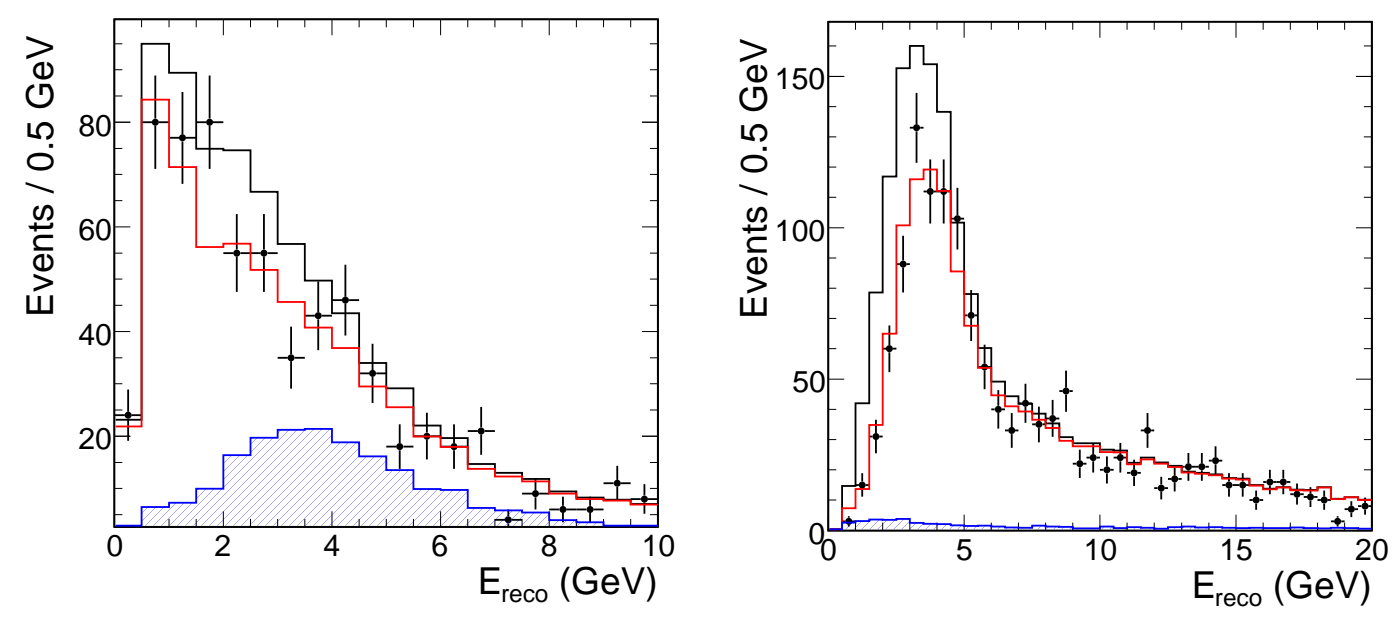

Figure 4.6: Far detector energy spectra for selected NC (left) and CC (right) events. The black lines show the unoscillated Monte Carlo prediction, the red lines the best fit spectra. The blue, hatched distribution shows the background due to misidentification. The black markers show the mock data set with statistical errors.

is oscillated according to the probabilities (4.2) and (4.3) and then subsequently fitted to the Far Detector data. The charged current and neutral current neutrino spectra are fitted simultaneously. The likelihood function described in Section 4.3 is again used to perform a MINUIT fit.

The fit used the selected charged current spectrum from 0 to $20 \mathrm{GeV}$ and the selected neutral current spectrum from 0 to $10 \mathrm{GeV}$. The fit parameters were the mass-squared difference $\Delta m_{32}^{2}$, the PMNS parameter $\sin ^{2}\left(2 \theta_{23}\right)$, and the sterile fraction $f_{s}$. The parameters $\sin ^{2}\left(2 \theta_{23}\right)$ and $f_{s}$ were constrained to the physically meaningful interval $[0,1]$; the parameter $\Delta m_{32}^{2}$ was allowed to vary freely.

\subsection{Results}

Data and Monte Carlo spectra as well as the spectra for the best fit to neutrino oscillations are shown in Figure 4.6. The resulting parameters with their $1 \sigma$ errors as returned by MINUIT are: 

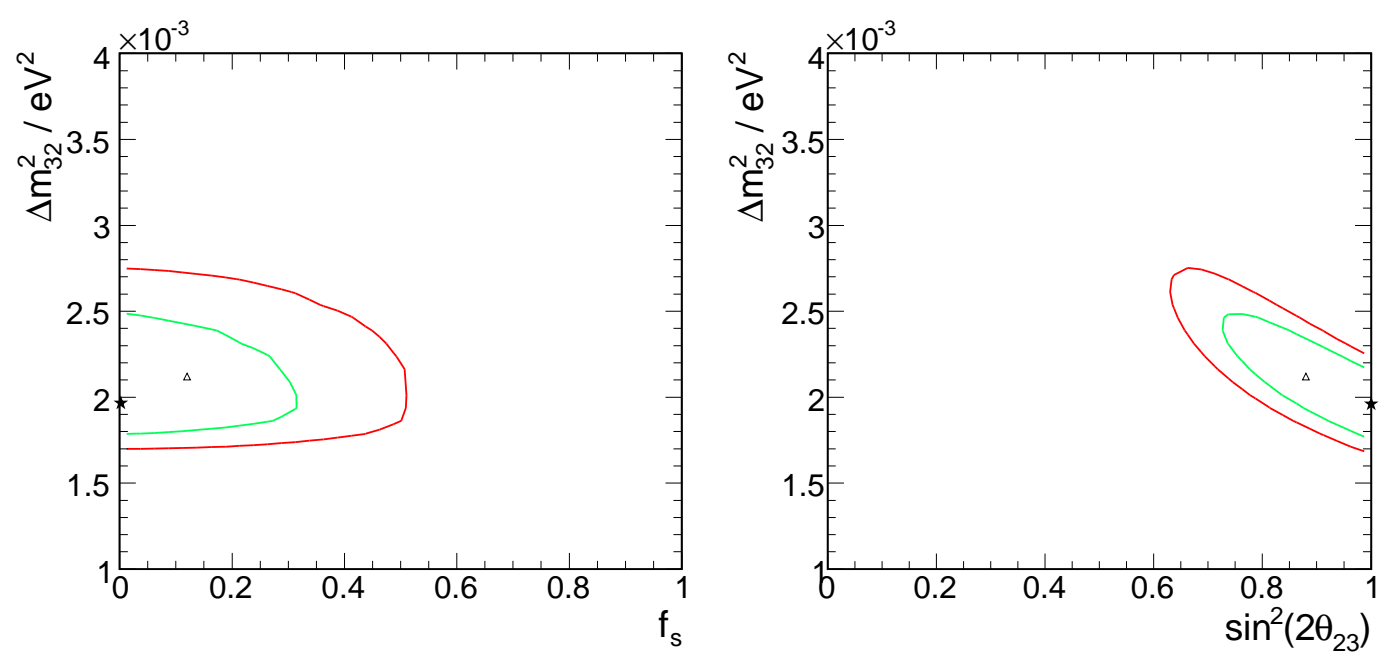

Figure 4.7: Best fit point and confidence contours for the Mock Data Challenge fit in the $\left(f_{s}, \Delta m_{32}^{2}\right)$ plane (left) and the $\left(\sin ^{2}\left(2 \theta_{23}\right), \Delta m_{32}^{2}\right)$ plane (right). The green lines shows the $90 \%$, the red lines the $99 \%$ confidence level contours, calculated from the rise in the likelihood function. The third fit parameter has been allowed to vary at every point in the plotted $2 \mathrm{D}$ projections. The star markers show the location of the best fit point, the open triangles the input values.

- $\Delta m_{32}^{2}=1.96_{-0.09}^{+0.20} \times 10^{-3} \mathrm{eV}^{2}$

- $\sin ^{2}\left(2 \theta_{23}\right)=1.00_{-0.12}^{+0.00}$

- $f_{s}=0.00_{-0.00}^{+0.09}$

The best fit is consistent with a maximal mixing scenario and a sterile fraction of zero. Since the best fit parameters for $\sin ^{2}\left(2 \theta_{23}\right)$ and $f_{s}$ are at the physical limit, only one-sided errors are quoted. The errors on these two parameters are sizable. A sterile fraction $f_{s}$ of 0.5 is still inside the $99 \%$ confidence region. The $90 \%$ and $99 \%$ confidence level contours for the fit are shown in Figure 4.7.

\subsubsection{Input values for the mock data set generation}

Since this was a simulated data exercise, it is possible to answer the question about the real values for the measured parameters. After all analysis working groups had announced their results at a MINOS collaboration meeting, the parameter values 
used to generate the mock data sets were revealed. The NEUGEN cross-section parameters are:

- $M_{A, \text { res }}=1.060 / 1.032=1.027$

- $M_{A, q e}=1.012 / 1.032=0.981$

- $K N O_{1 j k}=1.025$

The oscillation parameters are:

- $\Delta m_{32}^{2}=2.12 \times 10^{-3} \mathrm{eV}^{2}$

- $\sin ^{2}\left(2 \theta_{23}\right)=0.88$

- $f_{s}=0.12$

The measured best fit values for the NEUGEN cross-section parameters are in very good agreement with the generated input parameters. They are, in fact, consistent within one standard deviation. The extracted oscillation parameters too are consistent with the input values of the Monte Carlo experiment. The true values lie well within the $90 \%$ confidence contours of the fit.

\subsection{Conclusion}

In this chapter, we have described a preliminary study, which performed a complete analysis chain on a set of simulated data. A simple selection algorithm for neutral current events was developed and applied to both Near and Far Detector data samples. The selected neutral current events in the Near Detector were used to find improvements to the MINOS Monte Carlo simulation. Since no significant departures from the nominal values for the Monte Carlo parameters were found, the nominal Monte Carlo was used to fit the Far Detector sample for oscillations.

A 3-parameter fit for oscillations into $\nu_{\tau}$ and $\nu_{\text {sterile }}$ was developed and applied to the Far Detector data. The extracted results were consistent with the input values to the mock data generation. 
The Mock Data Challenge was an important procedure using both MINOS detectors and exercising the whole neutral current analysis chain. A complete analysis, starting with event reconstruction and selection and ending with a fit for sterile neutrinos was successfully performed.

In the following chapter, we will revisit the neutral current event selection with the goal of improving on the simple procedure described in this chapter. Two separate selection algorithms, one based on a succession of cuts and one using artificial neural networks are described. 


\section{Chapter 5}

\section{Selecting Neutral Current}

\section{neutrino interactions in the Near}

\section{Detector}

This chapter describes the process of selecting a clean sample of neutral current neutrino interactions in the MINOS Near Detector. It starts with the rejection of different backgrounds and subsequently presents two separate classification methods distinguishing neutral current from charged current neutrino interactions. The first method is based on a simple set of selection cuts whereas the second makes use of artificial neural networks (ANNs).

\subsection{Backgrounds and "Data cleaning"}

In this section, we will describe important backgrounds to the neutral current event selection and how to eliminate or at least significantly reduce them. We broadly divide the backgrounds into three categories: reconstruction failures, instrumental effects and misclassification. The first category includes cases where the reconstruction algorithms fail to identify the topology of the neutrino interac- 


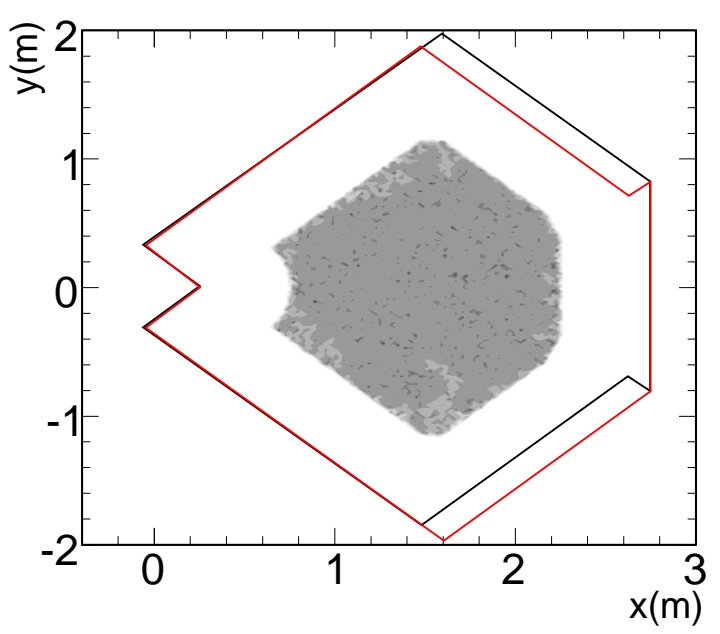

Figure 5.1: $\mathrm{x}-\mathrm{y}$ projection of fiducial event vertex positions. The lines show the outline of the partially instrumented planes. Black corresponds to the U-view and red to the $\mathrm{V}$-view. The $50 \mathrm{~cm}$ distance to the edge is clearly visible.

tion in the detector, split or merge events within a beam spill, move the location of the interaction in the detector or simply miss part of the energy deposition. The second category is due to an effect in the amplification stage of the multi-anode PMTs used in MINOS resulting in late pulses. The last category is made up from well reconstructed charged current events which are misclassified as neutral current interactions.

All but the last categories are specific to the Near Detector with its high rate of neutrino interactions and continuous readout for the duration of the beam spill. If these events are not dealt with, they would contribute to a systematic difference between the two MINOS detectors. It is therefore important to eliminate these events from the data sample.

\section{Fiducial Cut}

All plots shown in this section have fiducial cuts applied. For events to be selected, the vertex needs to be a minimum of $50 \mathrm{~cm}$ away from the closest edge of a partial plane outline. This ensures good shower containment and reduces the number 
of events in the sample which interacted outside the detector. For events with good tracks (i.e. where the track protrudes from the shower rather than being completely contained in it), the track vertex is used, otherwise the event vertex is used. Figure 5.1 shows an $\mathrm{x}-\mathrm{y}$ projection of fiducial events with the partial plane outlines superimposed. Longitudinally, a cut of $1.7 \mathrm{~m}<z<4.7 \mathrm{~m}$ is used. This is sufficiently far away from all edges to guarantee good shower containment.

\subsubsection{Reconstruction failures}

Reconstruction failures form an important background to NC analyses in MINOS. As a measure for these failures the quantity event completeness, or completeness in short, is used throughout the text. Completeness is defined as the summed reconstructed pulse height in the event attributed to the neutrino interaction using the Monte Carlo truth information divided by the total reconstructed pulse height due to this neutrino interaction. By definition, it can only be calculated for Monte Carlo events.

Around $8 \%$ of all reconstructed events in the fiducial volume have a completeness of less than 0.5 as shown in Figure 5.2. Of these low completeness events, $\sim 75 \%$ are classified as neutral current interactions by typical NC selection cuts resulting in a sizable background to the $\mathrm{NC}$ energy spectrum at low energies. Since only a small fraction of the true pulse height of these events is recorded, the reconstructed energy of low completeness events will be systematically too low. In addition, some of these events are completely pathological, and so are unlikely to be well modelled in the Monte Carlo simulation. Any classification method using the topology of hits in such events will be adversely affected by these badly reconstructed events.

Several different mechanisms which cause low completeness events have been identified. This effect is present in both real data and Monte Carlo simulation. It 


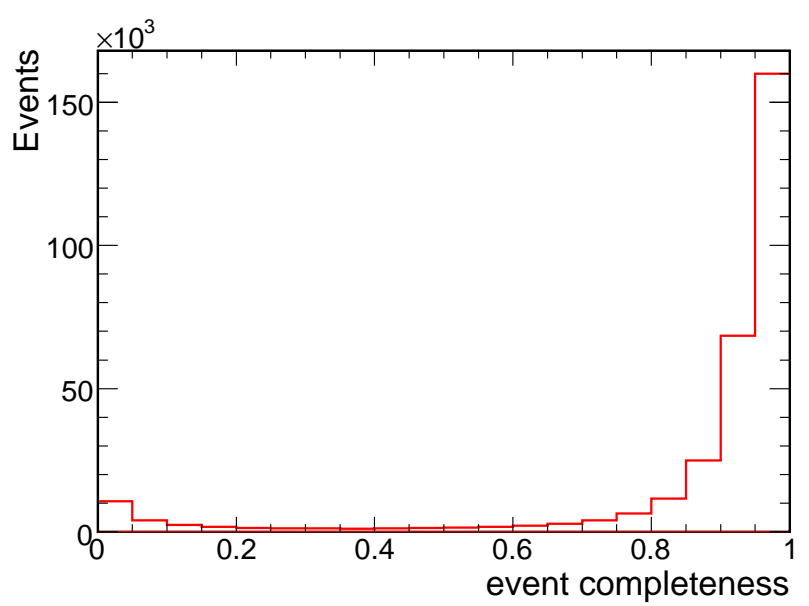

Figure 5.2: Event completeness variable in Monte Carlo. There is a sizable number of events with a completeness of less than $50 \%$.

is known however that the Monte Carlo does not completely describe the data in all the investigated cases. In the sections to follow, each of these mechanisms is described in turn and a set of cuts designed to remove these badly reconstructed events from the data sample is proposed. This process is referred to as data cleaning.

All plots showing both data and Monte Carlo distributions are normalised to the number of protons-on-target in the data sample. To make the effect of low completeness events more visible, only events with a reconstructed energy $E_{\text {reco }}$ below $5 \mathrm{GeV}$, and with either no track or a track which has less planes than the shower $\left(p_{t r k}<p_{s h w}\right)$, are shown in data/MC comparisons of individual variables. Events above $5 \mathrm{GeV}$ and events with an obvious track do not contribute to the low completeness background.

The Monte Carlo distributions are re-weighted to a tuned model for the production of hadrons in the NuMI target. Essentially, the parent pions, which decay to produce neutrinos, are weighted up/down in bins of longitudinal and transverse momentum, in order to make the Near Detector charged current energy spectrum agree with the measured data. This is done in a simultaneous fit to multiple dif- 

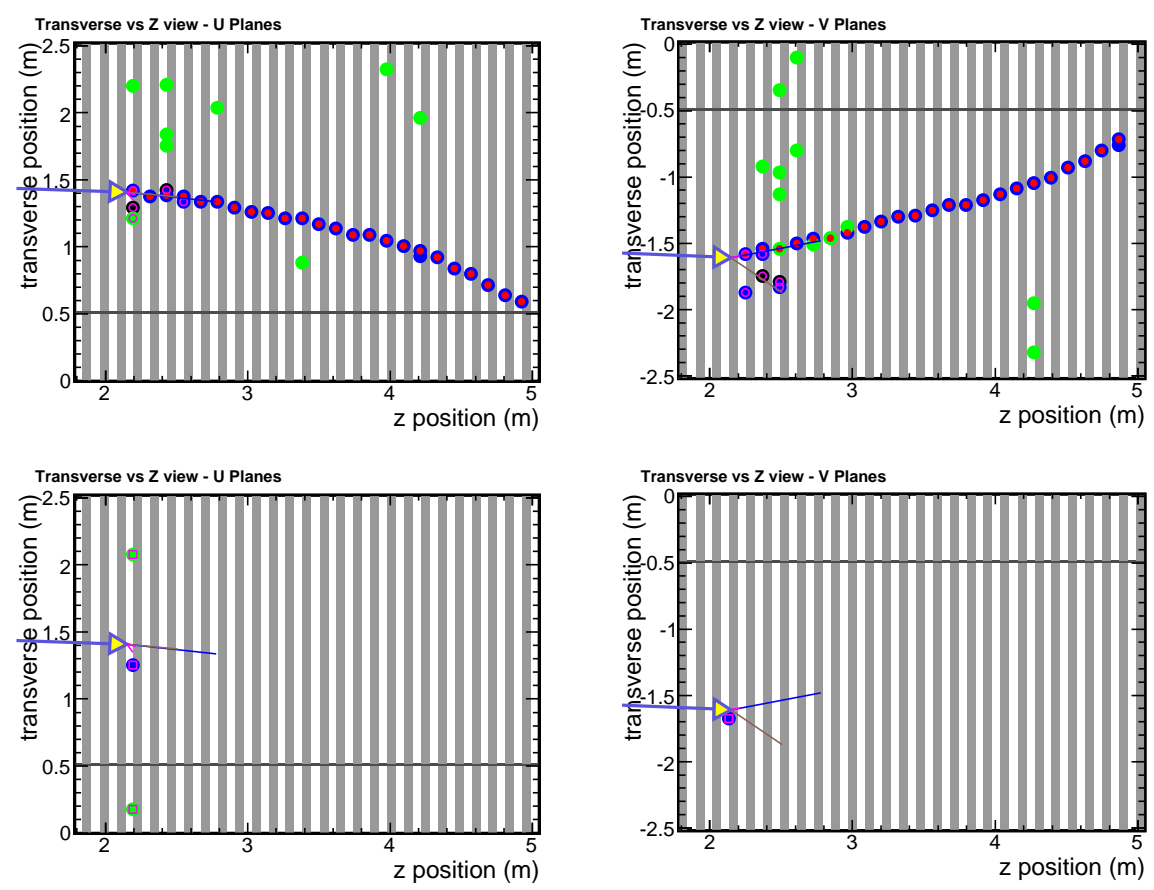

Figure 5.3: Example of a split event. The top two figures show the $\mathrm{U}$ and $\mathrm{V}$ views for the main event reconstructed for a given neutrino interaction in the Monte Carlo. The bottom two figures show a small second event near the vertex reconstructed from the same neutrino interaction. The time difference between the events is $36 \mathrm{~ns}$.

ferent beam configurations. The process of neutrino flux tuning is explained in more detail in the context of the $\nu_{\mu}$ disappearance analysis in section 7.2.

\section{The different failure modes}

The reconstruction failures can be divided further into three distinct categories: split events, vertexing failures, and everything else. Split events occur when a single neutrino interaction results in two or more reconstructed events. Vertexing failures cause neutrino interactions at the edge or outside the detector volume to be reconstructed with a vertex inside the fiducial volume and therefore to be included in the sample. The remaining "everything else" category includes cases where the reconstruction for a variety of reasons only includes a subset of the strips in the shower. 
Split events There are multiple different causes for an event to be split by the reconstruction. Late activity caused by neutrons or PMT afterpulsing can introduce large time gaps which cause events to be split during the slicing stage (see section 3.7 for an overview of the reconstruction chain). If there are gaps in the hit strip pattern, the shower reconstruction sometimes reconstructs multiple showers. In the case of a shower along the track, e.g. caused by $\delta$-rays, the reconstruction may fail to associate the shower with the track, causing a separate event to be formed. An example of a split event is given in Figure 5.3.

Split events will lead to double-counting of neutrino interactions and bad energy resolution. It is possible to eliminate split events from the data sample by using timing and spatial separation. If two reconstructed events are caused by the same neutrino interaction, they are expected to appear close in time and/or in space. The former is expected in case of slicing or shower reconstruction failures whereas the latter would be the case for late activity. We therefore use the time separation $\Delta t$ between the two events closest in time, referred to as min $\Delta t$ and the distances in the $z$ direction between the vertices of the two events closest in time, $\Delta z$, to remove split events.

In order to calculate these quantities, an event time variable $t$ is defined using the median of strip times in a 5-plane-window, starting at the vertex. As described in chapter 3, the single hit time resolution in the Near Detector is $~ 19$ ns. Averaging over multiple hit strips close to the event vertex results in a somewhat better resolution for the time of a reconstructed event. Figure 5.4 shows distributions of min $\Delta t$ and $\Delta z$ for data and Monte Carlo. Two-dimensional plots for these quantities are shown in Figure 5.5.

It can be clearly seen that there is a high contribution of low completeness events at low values of both variables. The variables shown use the closest event in time, regardless of whether it was reconstructed in the fiducial volume. This serves to identify split events in the fiducial volume whose "partner" was reconstructed 

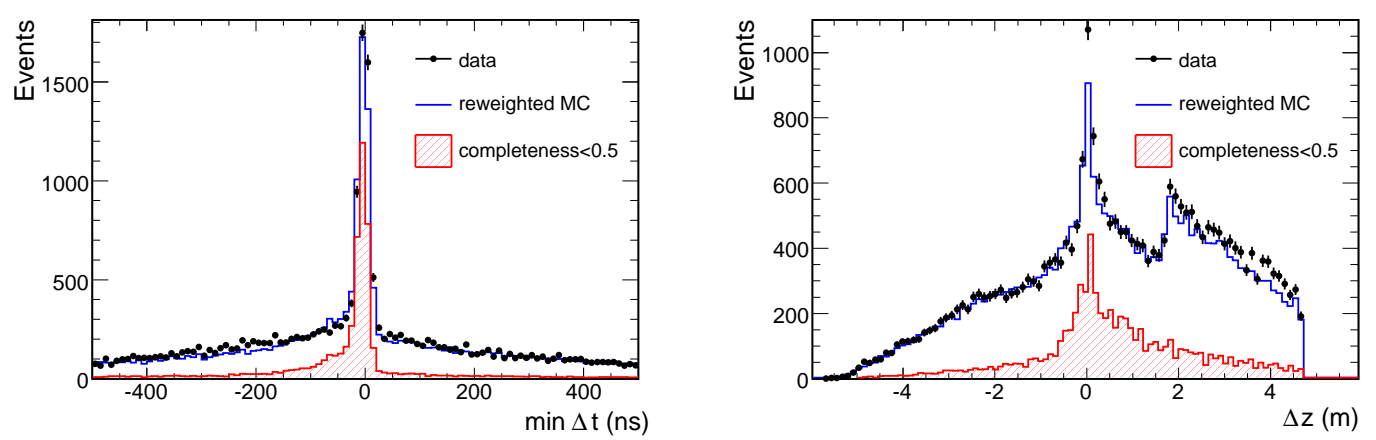

Figure 5.4: $\min \Delta t$ and $\Delta z$ variables for events with $E_{\text {reco }}<5 \mathrm{GeV}$ and $p_{\text {shw }}>p_{\text {trk }}$. The low completeness events are shown in the hatched histograms. There is a sizable contribution of low completeness events at small time and space separations. Low completeness events are more likely to occur later than their closest "partner" (negative min $\Delta t$ values).
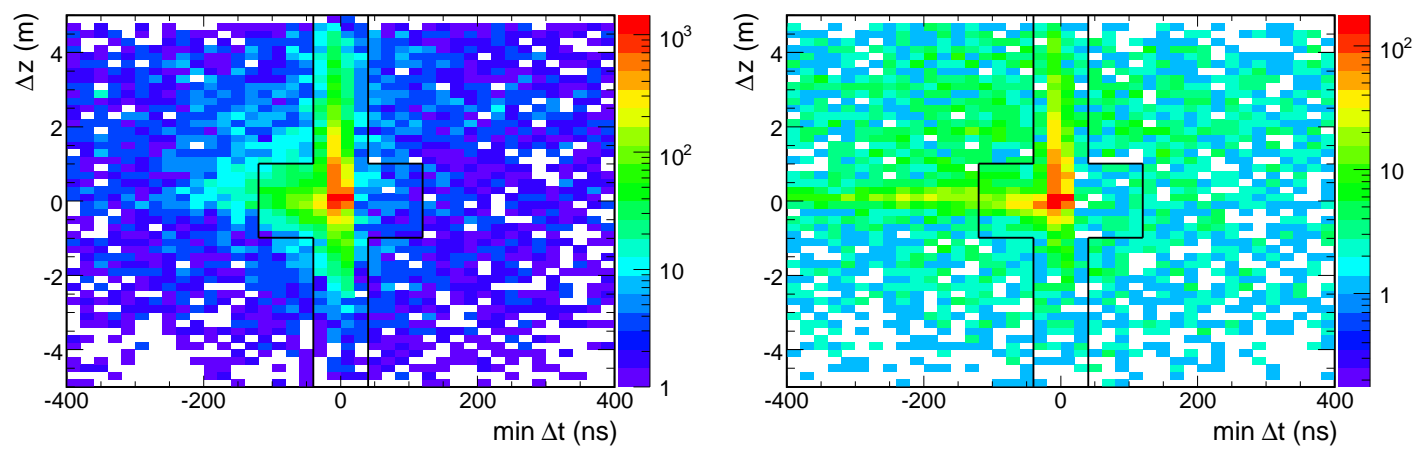

Figure 5.5: $\Delta z$ vs. min $\Delta t$ plots for low completeness events in Monte Carlo (left) and all data (right). Only events with $E_{\text {reco }}<5 \mathrm{GeV}$ are shown. Events inside the lines are removed from the sample. 

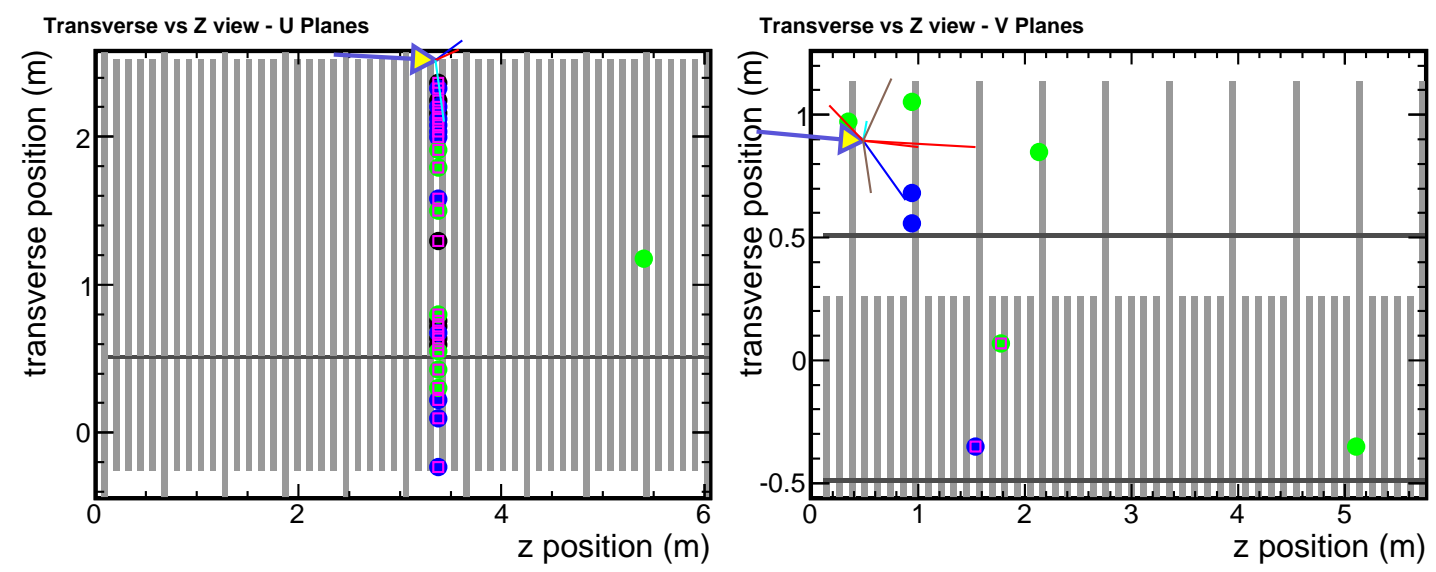

Figure 5.6: Two different example "leakage" events. Only one view is shown for each event. The left event consists of a very steep shower. The right event has considerable activity in the sparsely instrumented region of the near detector. Its reconstructed vertex is inside the fiducial volume.

outside the fiducial volume. Low completeness events are more likely to occur later than their closest "partner" (negative min $\Delta t$ values). The shape of the $\Delta z$ distribution is caused by a combination of the longitudinal fiducial cut and the fact that muons from neutrino interactions in the surrounding rock, entering the detector from the front, have a vertex coordinate of $\mathrm{z}=0 \mathrm{~m}$. The distance between a selected event and a rock muon entering from the front is therefore always between $1.7-4.7 \mathrm{~m}$.

The proposed cut values for all variables are shown on page 88, which shows $\mathrm{N}-1$ plots ${ }^{1}$.

Vertexing failures (so called "leakage") Events which occurred outside the fiducial volume but which are reconstructed inside the fiducial volume are referred to as "leakage" events. This happens because very steep showers cause problems for the vertex or shower algorithms or because non-fiducial events enter the detector via the sparsely instrumented regions at the side of the Near Detector. Figure 5.6 shows two examples of this. Not all of these events end up in the low

\footnotetext{
${ }^{1} \mathrm{~N}-1$ plots for a set of cuts show one variable with the cuts on all other quantities already applied.
} 
completeness sample. Because the denominator in the completeness definition is reconstructed strips, "leakage" events can have high values in this variable. Nevertheless, these events are missing a substantial amount of their energy and are often completely pathological. It is important to point out that this is not just a matter of the vertex moving by a small distance. Cases have been observed where the reconstructed vertex is wrong by several metres. A charged current event occurring outside the detector for example could be completely missing its track and just leave a small shower inside the detector volume.

Two distinct types of cuts are applied in order to remove these failure modes. They deal with "leakage" events, regardless of whether they are low completeness or not.

Firstly, a steepness variable $s$ is used to characterise very steep showers. Steepness is defined as:

$$
s=\frac{N_{\text {strips }} / p_{\text {evt }}}{p_{\text {evt }}} \quad \text { where } \quad p_{\text {evt }} \equiv \text { planes in event }
$$

This is similar to width divided by length of the shower but also takes into account whether a large number of strips are hit in a given plane. A high steepness value means the shower spans very few planes but has many strips hit. It is therefore more likely that the event is due to activity entering the detector from the side. The distributions for this variable are shown in Figure 5.7.

Secondly, it is possible to use the activity in the sparsely instrumented regions as a "leakage" veto. In order to do this, a veto region at the edges of the detector in both views is defined. Figure 5.8 shows the transverse extent of these veto regions. The summed pulse height and the number of hit strips in each of the two veto regions are cut on in order to further reduce the "leakage" contribution. The veto variables are called edgeStrips and edgePH for the bigger veto region shown in Figure 5.8 (a) and oppEdgeStrips and oppEdgePH for the opposite side of the 


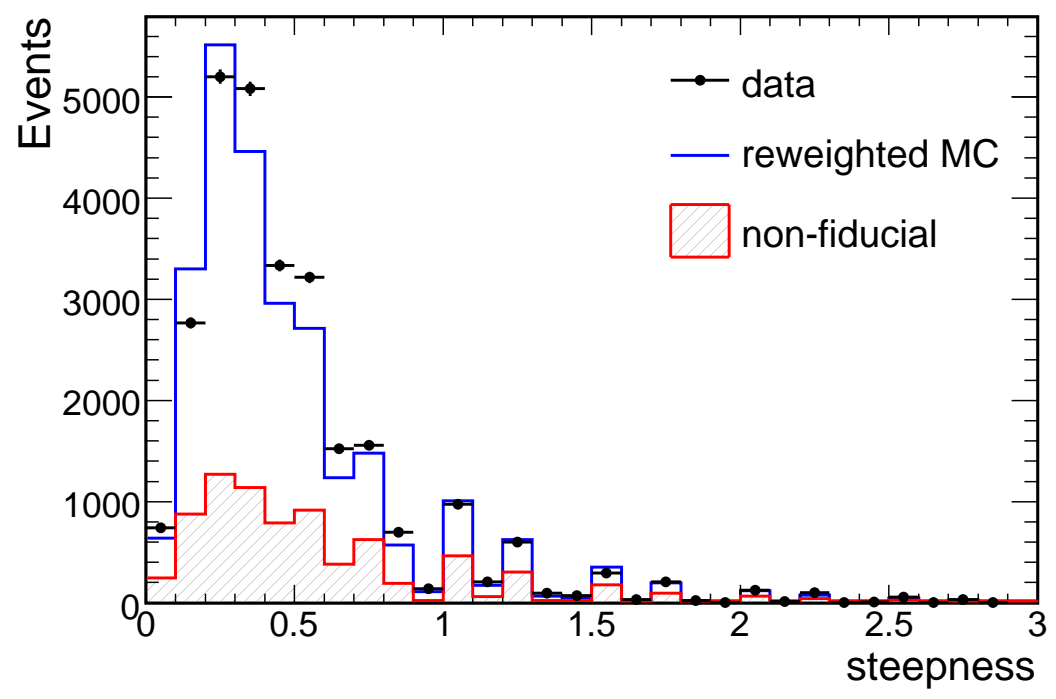

Figure 5.7: Steepness variable for events with $E_{\text {reco }}<5 \mathrm{GeV}$ and $p_{\text {shw }}>$ ptrk. Events with a true vertex outside the fiducial volume are shown in the hatched histograms. There is a larger contribution of "leakage" events at high steepness values.

detector. In order for an event to be discarded, a minimum number of strips and a minimum pulse height in the veto region is required. Only strips in the calorimeter region of the detector and within a $\pm 40 \mathrm{~ns}$ window around the event are used. Strips are not required to be reconstructed as part of the event; every strip in the correct space-time-window is counted. This makes the veto variables robust against slicing or shower reconstruction failures. Distributions for the veto variables are shown in Figure 5.9. The time window used is a trade-off between being short enough to count only strips which could have been caused by the neutrino interaction in question and being robust against bad timing. It is not fully optimised but works sufficiently well. These variables have recently been studied in more detail by R. Pittam [91].

Other reconstruction failures This final category is a catch-all category intended to deal with the remaining issues causing low completeness events. Among others, these are caused by the shower reconstruction not assigning all strips to 

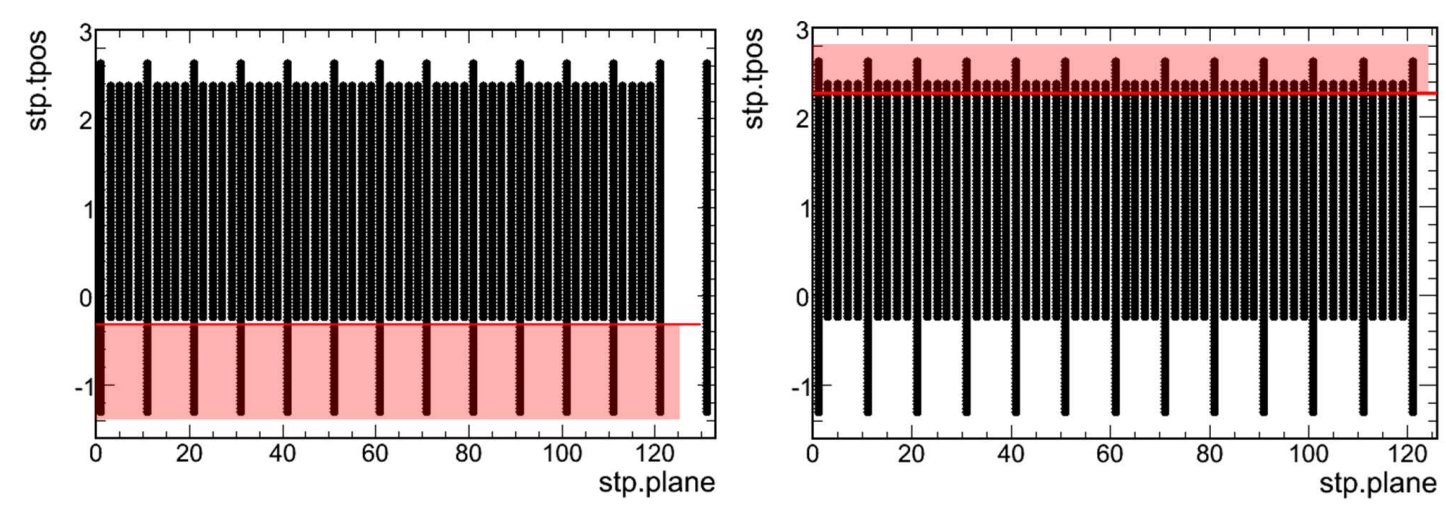

Figure 5.8: Veto regions. The veto region shown on the left defines the variables edgeStrips and edgePH, the right defines the variables oppEdgeStrips and oppEdgePH. The two veto regions are cut on separately, because an event will enter the detector from either one side or the other.
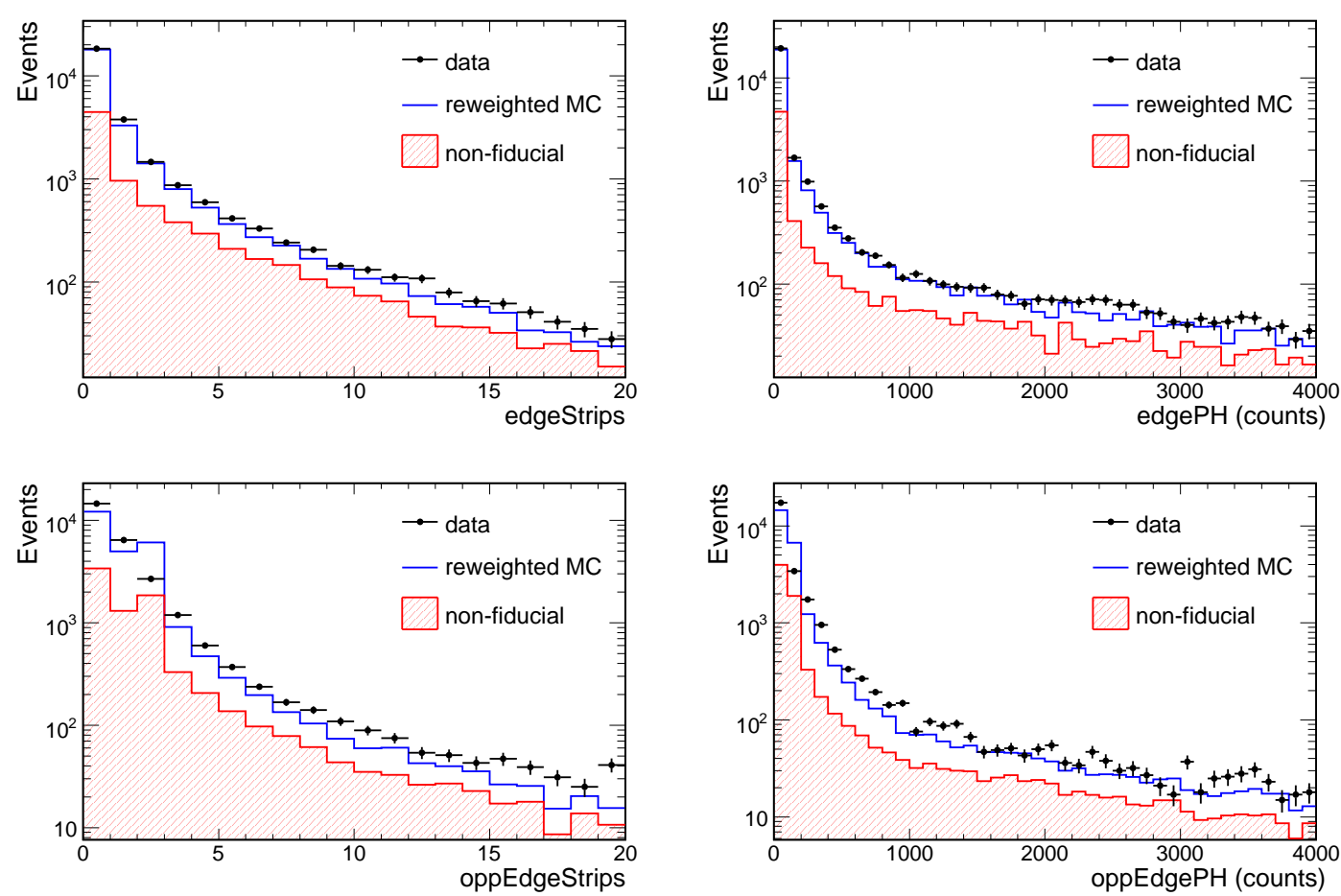

Figure 5.9: Veto variables. The top two figures show the number of hit strips and summed $\mathrm{PH}$ in the larger veto region (left of Figure 5.8). The bottom two figures show these variables for the other veto region. The hatched histograms show events with a true vertex outside the fiducial volume. High values in these veto variables mean that there was considerable activity in the edge regions of the detector. Events like this are more likely to have entered the detector from the side. 


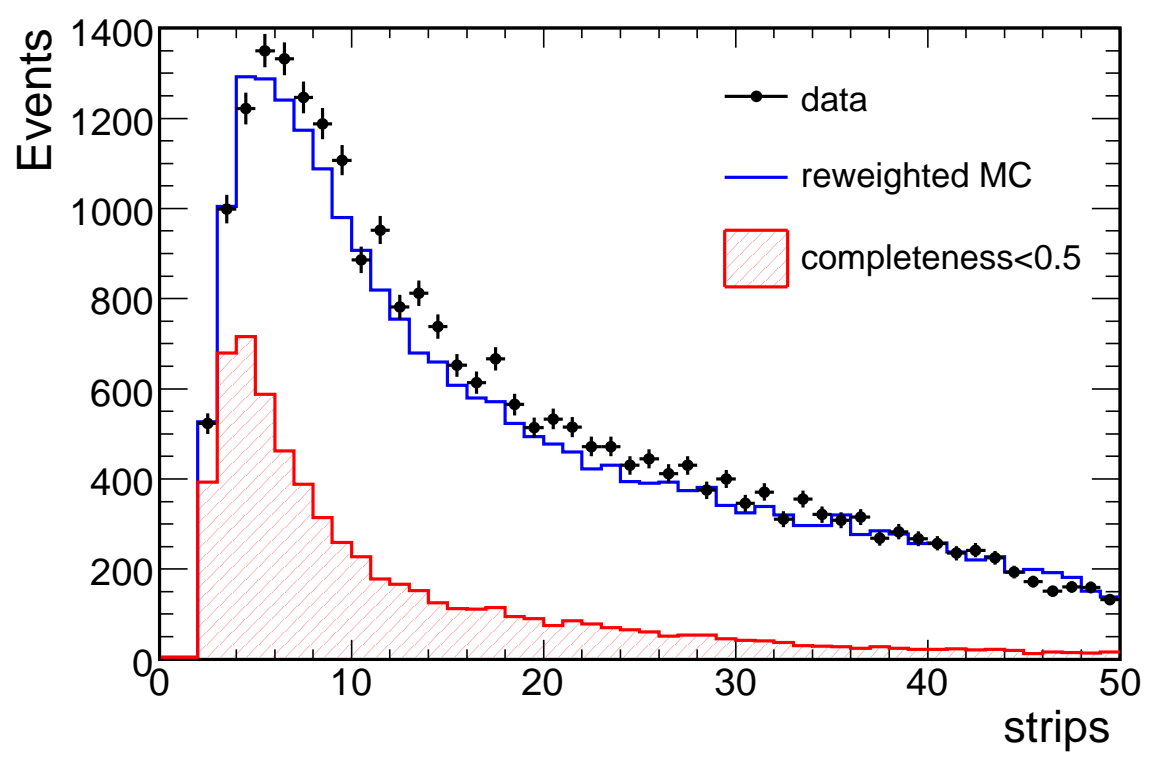

Figure 5.10: Number of strips in the event. The hatched histogram shows events with completeness less than 0.5. There is a high contribution of badly reconstructed events at low strip counts.

the shower. This happens if there are large gaps in a shower or if the shower is generally sparse. Variables characterising the spatial extent and density of the shower have been investigated but were found not to be useful in dealing with these failures. Instead, the observation that the majority of these cases have a very low reconstructed strip count, as shown in Figure 5.10, is used.

\section{Proposed Cuts and N-1 plots}

In this section, we summarise the proposed set of cuts and show $\mathrm{N}-1$ plots for the quantities involved.

In order for an event to remain in the data sample, the following conditions have to be satisfied:

For all events:

- time separation between events: $|\min \Delta t|>40 \mathrm{~ns}$ 

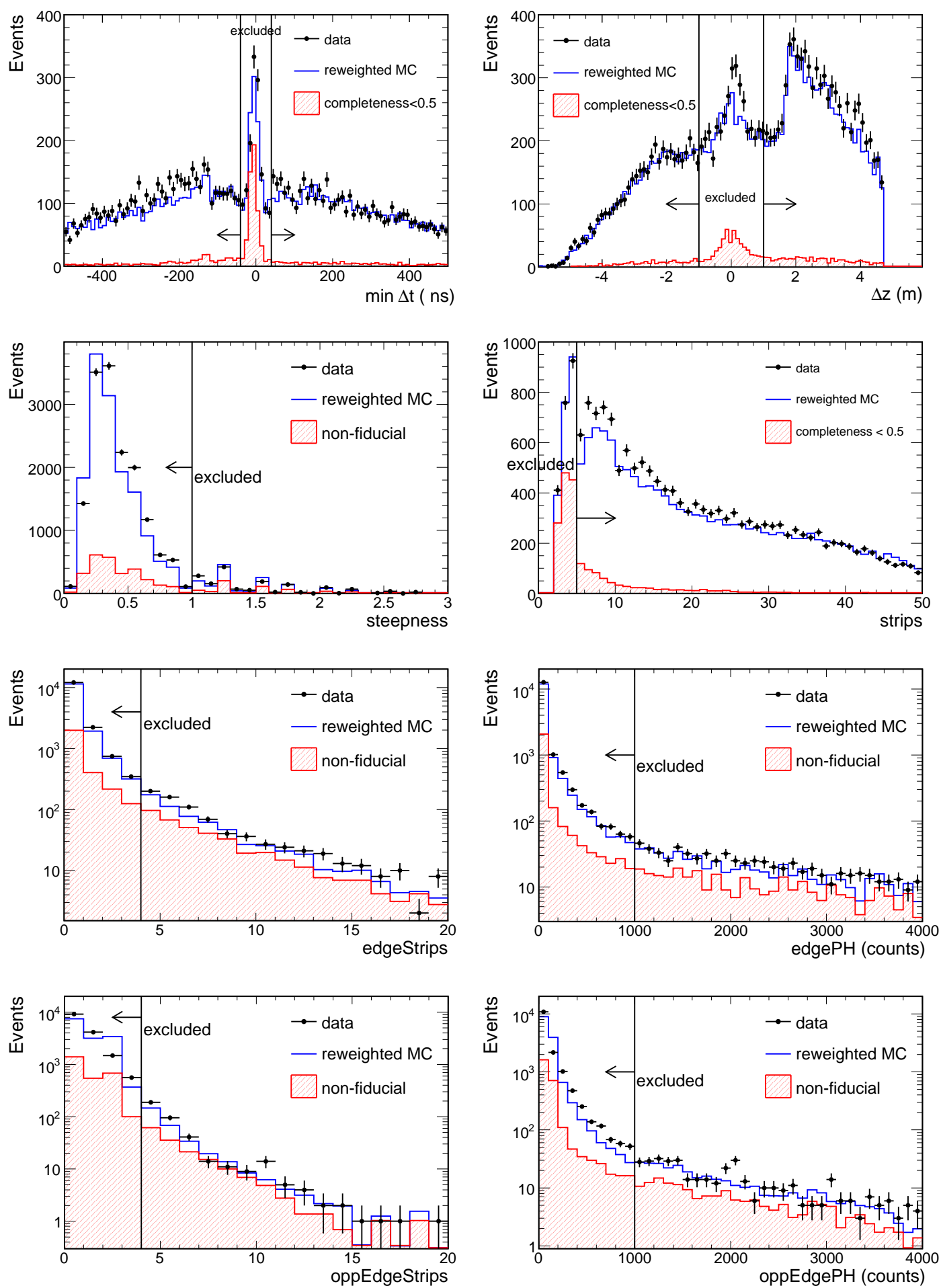

Figure 5.11: N-1 plots for the cut variables. Depending on whether the variable shown is meant to cut split or otherwise badly reconstructed events, or "leakage" events, the hatched histogram either shows low completeness events or non-fiducial events. The line and arrow indicate which side of the cut remains in the sample. 
- spatial separation: IF (40 ns $<|\min \Delta t|<120 \mathrm{~ns})$ : $|\Delta z|>1 \mathrm{~m}$

- steepness: $s<1.0$

- number of strips: $N_{\text {strips }}>4$

For events with a $E_{\text {reco }}<5 \mathrm{GeV}$ and with $p_{\text {shw }}>p_{\text {trk }}$ :

- $($ edgeStrips < 4) OR (edgePH $<1000$ counts)

- (oppEdgeStrips < 4) OR (oppEdgePH < 1000 counts)

The first four cuts in the list are applied to all events. The veto cuts (last two bullet points) are only applied to events with a reconstructed energy below $5 \mathrm{GeV}$ and where there is no sizable track $\left(p_{s h w}>p_{t r k}\right)$. This makes the cuts more specific to "leakage" events while retaining a good overall efficiency, since there is no low completeness contribution above $5 \mathrm{GeV}$. The spatial separation cut is only applied in a time window since otherwise there are too many random associations of events which do not originate in the same neutrino interaction.

The N-1 plots for the different distributions are shown in Figure 5.11. Each N-1 plot has all cuts but the one shown applied to it. This takes into account correlations between the variables and shows the power each cut adds to the "cleaning" procedure.

\section{Results}

Figure 5.12 shows the reconstructed energy spectrum for a neutral current event selection. The left spectrum is shown without the cleaning cuts; the right spectrum is shown after cuts. It is clearly visible that the low completeness component (shown in magenta) is significantly reduced.

Table 5.1 shows neutral current selection efficiencies and purities with and 

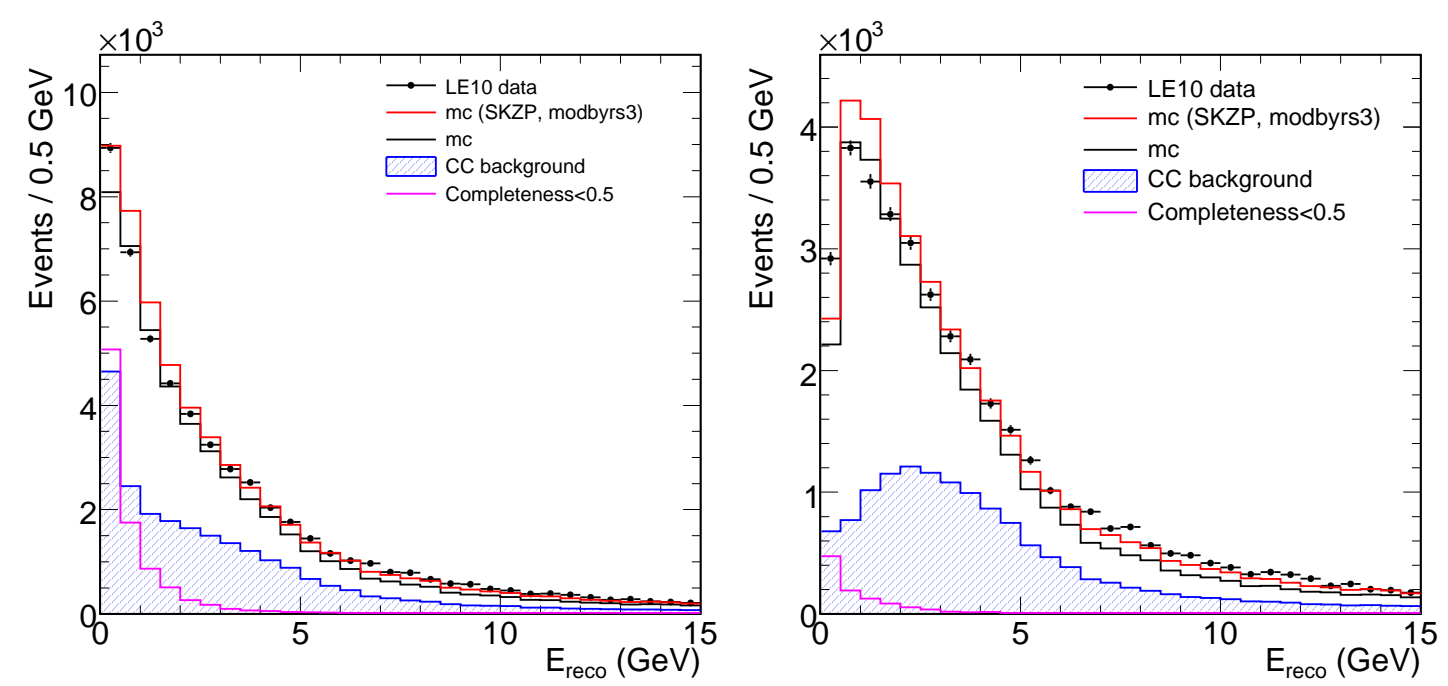

Figure 5.12: Neutral current energy spectra before (left) and after (right) cleaning cuts applied. The red and black lines show the Monte Carlo with and without re-weighting. The blue histogram shows the charged current background. The distribution in magenta shows the low completeness background. It is significantly reduced by the proposed cuts.

without the proposed data cleaning cuts ${ }^{2}$. The numbers are for a Monte Carlo sample of $1.5 \times 10^{19}$ protons on target. A specific neutral current event selection which will be described in detail in section 5.3 is used for this. Graphs of efficiencies and purities as a function of energy are shown in Figure 5.13.

Agreement between the data and Monte Carlo distributions used in this procedure is generally good. The number-of-strips distribution seems to be shifted between data and Monte Carlo. The cut on this variable therefore contributes to the systematic uncertainty of this method.

\subsubsection{An instrumental effect: PMT afterpulsing}

During the course of this work, an instrumental effect in the recorded Near Detector data was discovered. It manifests itself as an excess of reconstructed events

\footnotetext{
${ }^{2}$ Efficiency and purity are defined w.r.t. the sample in the fiducial volume, i.e. $100 \%$ efficiency means every fiducial NC event is selected. This does not include reconstruction efficiencies and losses due to fiducial cuts.
} 


\begin{tabular}{|l|r|r|r|r|r|r|}
\hline no selection & $0-2 \mathrm{GeV}$ & $\%$ & $2-5 \mathrm{GeV}$ & $\%$ & $>5 \mathrm{GeV}$ & $\%$ \\
\hline \hline Fiducial Events & 73740 & 100 & 123783 & 100 & 146345 & 100 \\
\hline Events after cleaning & 38652 & 52 & 98505 & 80 & 106591 & 73 \\
\hline \hline NC selection without cleaning \\
\hline Efficiency & $97 \%$ & \multicolumn{3}{|c|}{$88 \%$} & \\
\hline Purity & $57 \%$ & & $51 \%$ & $54 \%$ \\
\hline \hline NC selection after cleaning & $65 \%$ & & $74 \%$ & \\
\hline Efficiency & $73 \%$ & $53 \%$ & & $51 \%$ \\
\hline Purity &
\end{tabular}

Table 5.1: Efficiencies and purities before and after applying the data cleaning cuts. Efficiency and purity are defined with respect to the sample in the fiducial volume. A specific NC selection is used but results are expected to be very similar between different selection methods. The purity of the selected sample is significantly increased after the cleaning cuts, especially at low energies.
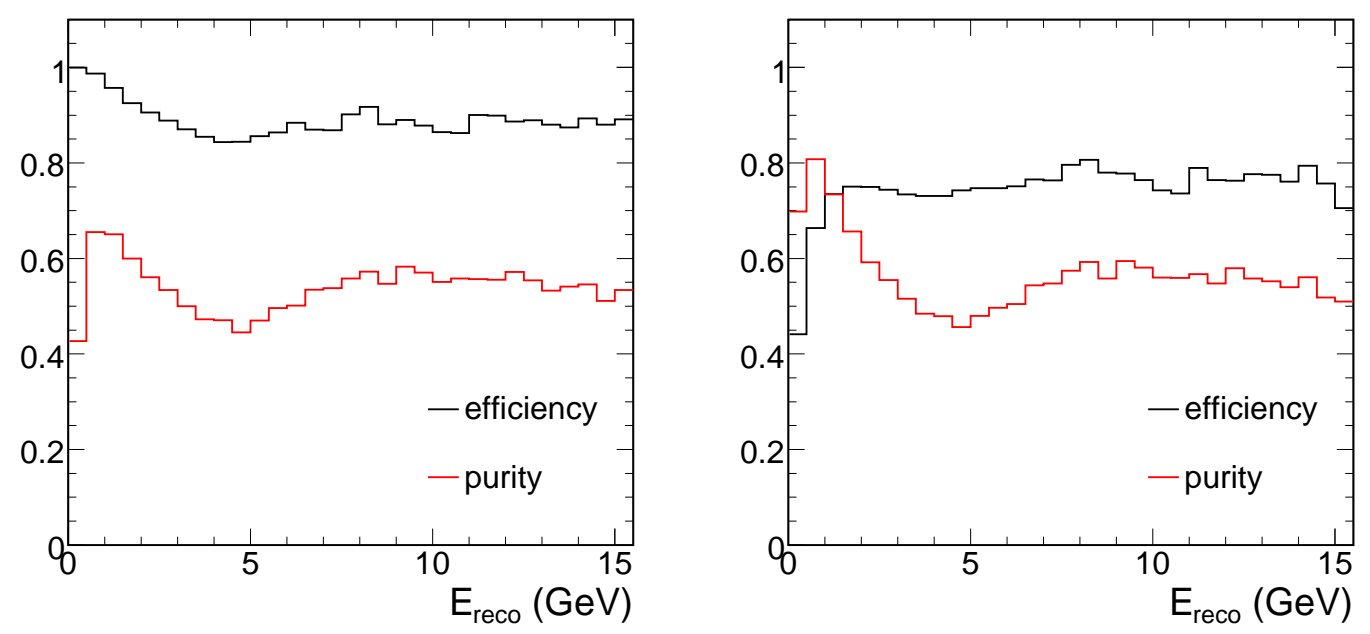

Figure 5.13: Efficiencies and purities before (left) and after (right) applying cleaning cuts. The purity of the selected sample is significantly increased after the cleaning cuts. 
with very low energies. A comparison of event energies in data and Monte Carlo is shown on the left of Figure 5.14. Data and Monte Carlo agree well, except in the first bin where a large excess in the data is observed.

It was found that these low energy events are primarily formed from strips mapping onto edge pixels of the Hamamatsu M64 photomultiplier tubes used in the Near Detector. The most likely cause is therefore an instrumental effect, described for example in [92]. It is referred to as "afterpulsing".

There is a non-zero probability that a signal in a PMT produces a smaller, secondary hit in the same PMT on a timescale of several hundred nanoseconds. Preferentially, this secondary hit occurs on the same pixel as the previous activity. There is however some evidence [93] that afterpulsing activity in the edge pixels can be caused by earlier hits in neighbouring pixels.

The underlying cause for afterpulsing is the ionisation of residual gas inside the PMT. These ions can subsequently interact with the photocathode and start a new cascade leading to a secondary pulse. The geometry of the dynode system provides a free path for the ions to reach the photocathode only along the edges. This explains the observed preference of afterpulsing for the edge pixels of a PMT.

Further evidence for the effect of afterpulsing can be seen in Figure 5.14, which shows the charge-weighted times of reconstructed strips with respect to the start of the neutrino spill. After the end of the neutrino spill at around $11 \mu \mathrm{s}$, there is a clear excess of reconstructed strips over the baseline visible in the first microsecond. It was possible to attribute this effect to the PMTs by studying the distribution of late hits in PMT pixel space. Strips with $t-t_{0}>11.5 \mu \mathrm{s}$ are almost solely attributable to late activity. Grouping the strips according to their position on the face of the PMT shows a prominent pattern (see Figure 5.15): The edges of the PMT face have more late activity than the central pixels.

In order to cut out events which are formed from afterpulsing activity, we make use of the fact that afterpulsing preferentially occurs on the same pixel as the 

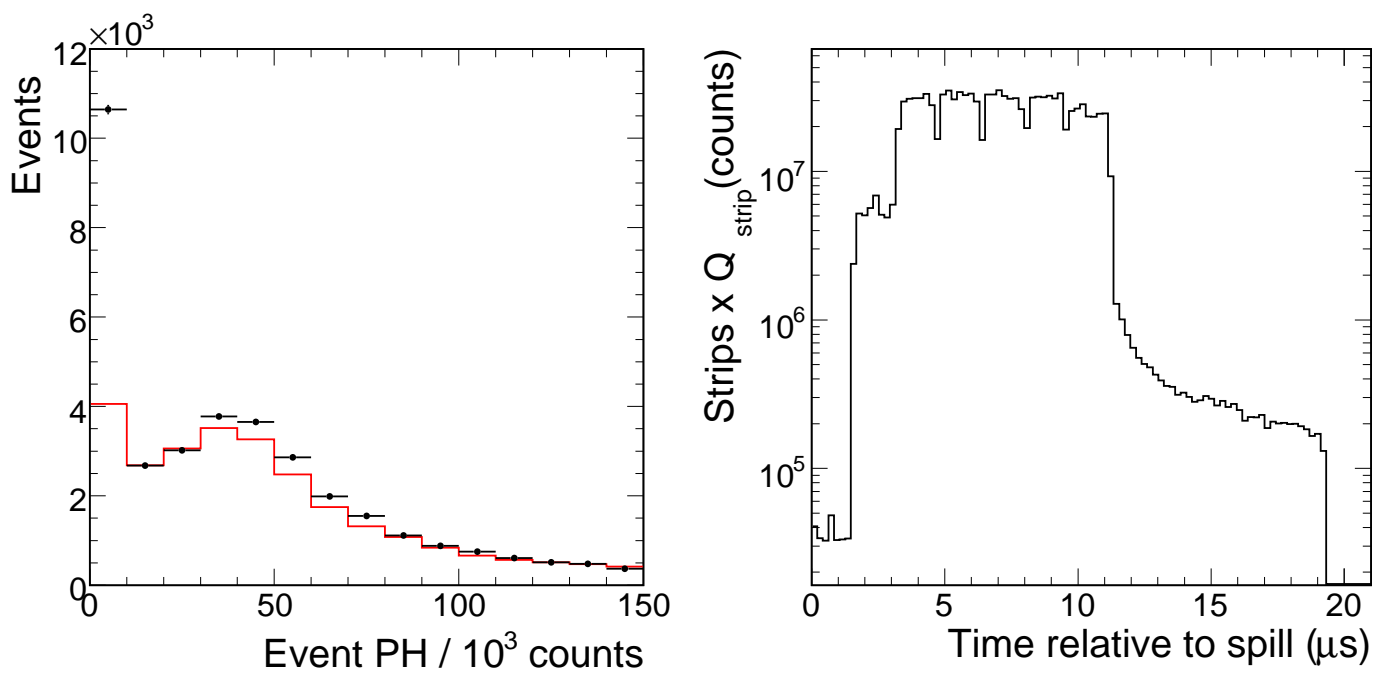

Figure 5.14: Event pulse height (left) and charge-weighted strip times in the spill window (right). The left figure shows event pulse height in data (points) and Monte Carlo (red line). There is an excess of low energy events in the data. The right figure shows strip times in the spill window. The excess events are reconstructed from strips with times later than the end of the neutrino spill $(\sim 11 \mu s)$. This is attributed to late activity or "afterpulsing" in the PMTs.
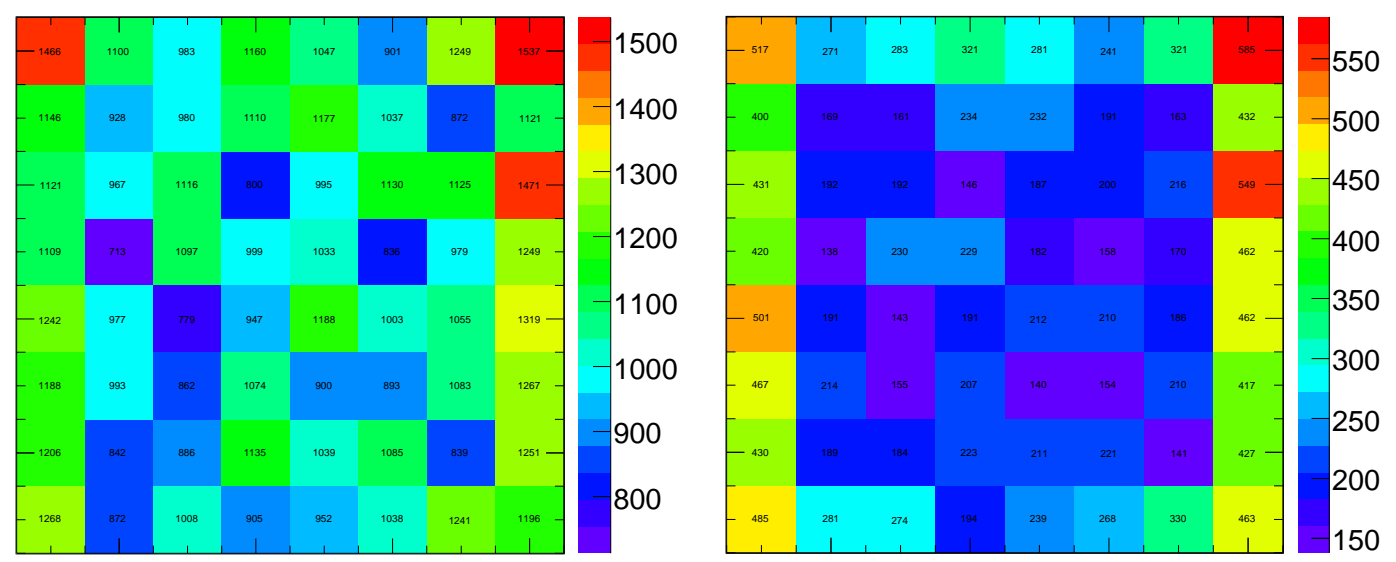

Figure 5.15: Position of late activity in pixel space. Late activity strips are selected by demanding $t-t_{0}>11.5 \mu \mathrm{s}$. The sum of multiple different PMTs is shown. The left plot shows all strips satisfying the time condition. The right plot shows only hits which occurred at least $300 \mathrm{~ns}$ after the first hit on the PMT. There is a clear excess of activity along the edges of the PMT. 
original pulse. After an energy deposition in a strip is reconstructed, an artificial dead-time of $2 \mu s$ is introduced in a given strip before new events can be formed. The interval of $2 \mu \mathrm{s}$ is motivated by Figure 5.16. This figure compares the time separation of hits in the same PMT pixel for data and Monte Carlo simulation. The excess events in data all cluster at low values of this time separation. After a strip-time separation of around $2 \mu s$, data and Monte Carlo simulation agree.

The characteristics of afterpulsing have been studied in detail in the recorded MINOS data. Attempts have been made to parameterise the afterpulsing probability in terms of a double-exponential function fitted to the tail of the time distribution shown in Figure 5.14. Every hit in a given PMT contributes to the probability of observing an afterpulsing hit on the same PMT later in time. Every event would then be assigned an afterpulsing score based on its temporal proximity to previous hits on the same PMT and the energy they deposited. This method would have the advantage of taking into account migration of afterpulsing into neighbouring pixels. After a detailed investigation this method however did not show any significant advantages over the simple timing cut introduced above and it was therefore not pursued any further.

\subsubsection{CC interactions}

The most important and most numerous background to any neutral current analysis are wrongly identified charged current interactions. This class of events will be the focus of the two subsequent sections on event selection.

\subsection{Cut-based selection}

After "data cleaning" and rejection of instrumental backgrounds, we will now present two separate event classification techniques. The goal of these procedures is to separate the neutral current neutrino interactions of interest to this analysis 


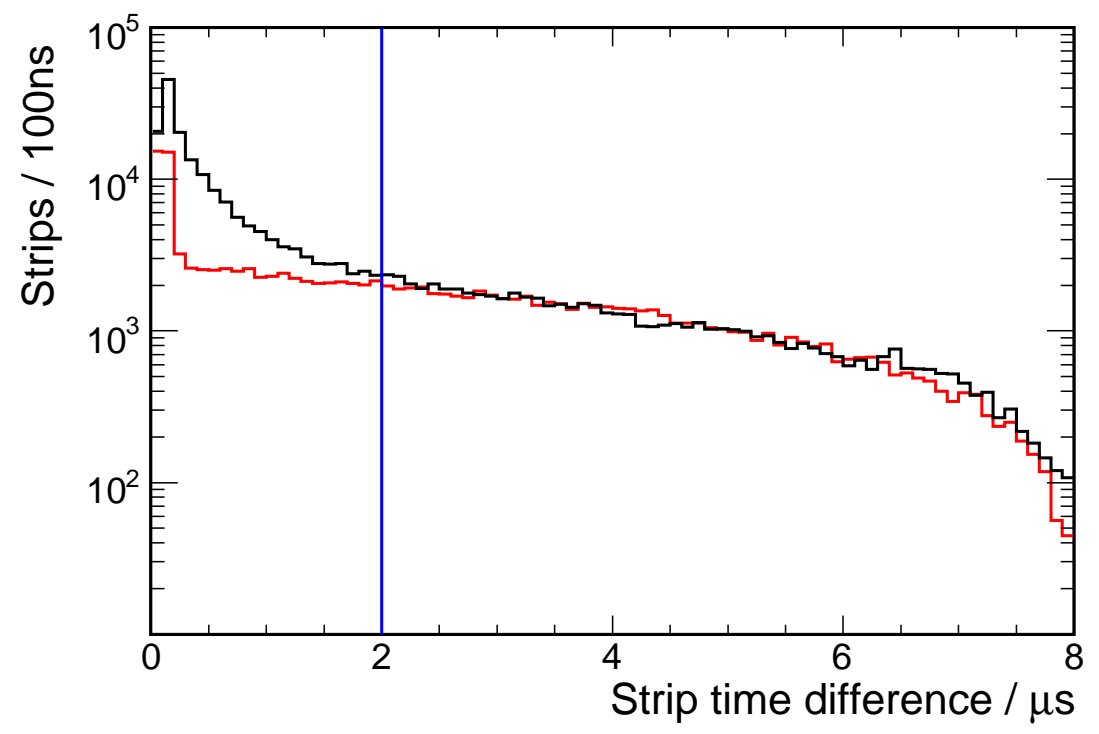

Figure 5.16: 2 $\mu s$-cut for afterpulsing. This plot shows the time separation between energy depositions on the same PMT pixel for pixels which were hit multiple times. The read line shows Monte Carlo, the black line data. The observed excess in data is concentrated at small time separations. From about $2 \mu s$ onward, data and Monte Carlo agree. Therefore, hits occurring closer than $2 \mu s$ to a previous energy deposition in the same pixel are excluded from the event reconstruction. 
from their main background, i.e. charged current neutrino interactions. First a simple, cut-based selection method will be presented. It employs the same variables as presented for the Mock Data Challenge analysis in chapter 4 but the placement of the cuts has been optimised further.

Subsequently, a more sophisticated event classification method using artificial neural network techniques will be laid out. Finally, the two methods will be compared and contrasted.

For the remainder of this chapter, all quantities will be shown after fiducial cuts, described in section 5.1, have been applied. This is true for both selection methods being presented.

\subsubsection{Event selection cuts}

As described in chapter 4, this simple cut-based selection tries to make use of the fact that neutral current events do not have a muon in the final state. The most distinguishing quantities are therefore the event length and, if present, some measure of track quality. It is not good enough to ignore events with a reconstructed track outright since $58 \%$ of all neutral current events in the fiducial volume have a track reconstructed. These tracks are either caused by energetic protons or pions in the hadronic shower or randomly associated shower hits which are grouped to form a track. Especially the latter class of events is susceptible to track quality cuts. If no track is present in an event, only event length is used for classification.

For subsequent use, we define the following quantities: the event length $\mathrm{L}$ measured in planes, the number of planes which the track extends out of the shower, referred to as track extension $\epsilon$, and the relative track fit error $\hat{\sigma}$ as returned from the fit.

In order to be classified as neutral current, an event has to satisfy the following conditions: 

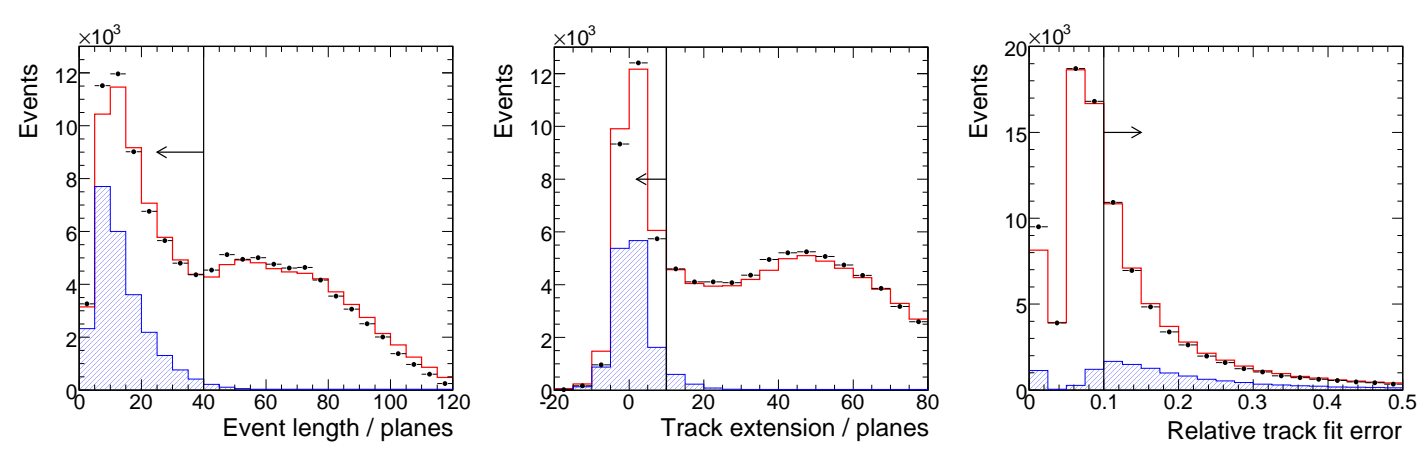

Figure 5.17: Selection variables. The three figures show the neutral current selection variables event length, track extension and relative track fit error. The red line and the black markers show Monte Carlo and data respectively. The hatched, blue distributions show the true neutral current events. The arrows indicate which side of the cut remains in the selected sample.

- $L<40$ planes

if a track is present:

- $\epsilon<10$ planes

- $\hat{\sigma}=\frac{\sigma(q / p)}{|q / p|}>0.1$

The distributions of these variables in data and Monte Carlo simulation are shown in Figure 5.17. Charged current events are on average longer, have a bigger value for the track extension and have smaller track fit errors.

\subsubsection{Resulting energy spectrum}

Despite its simplicity, the neutral current event selection based on three variables achieves very similar results to other, more sophisticated techniques. The overall selection efficiency for the cuts detailed above is $86 \%$ with a neutral current purity of $56 \%$.

Figure 5.18 shows the energy spectrum of selected neutral current events. Efficiency and purity curves as a function of energy for this selection will be shown in comparison with a more sophisticated event classification technique at the end of the following section. 


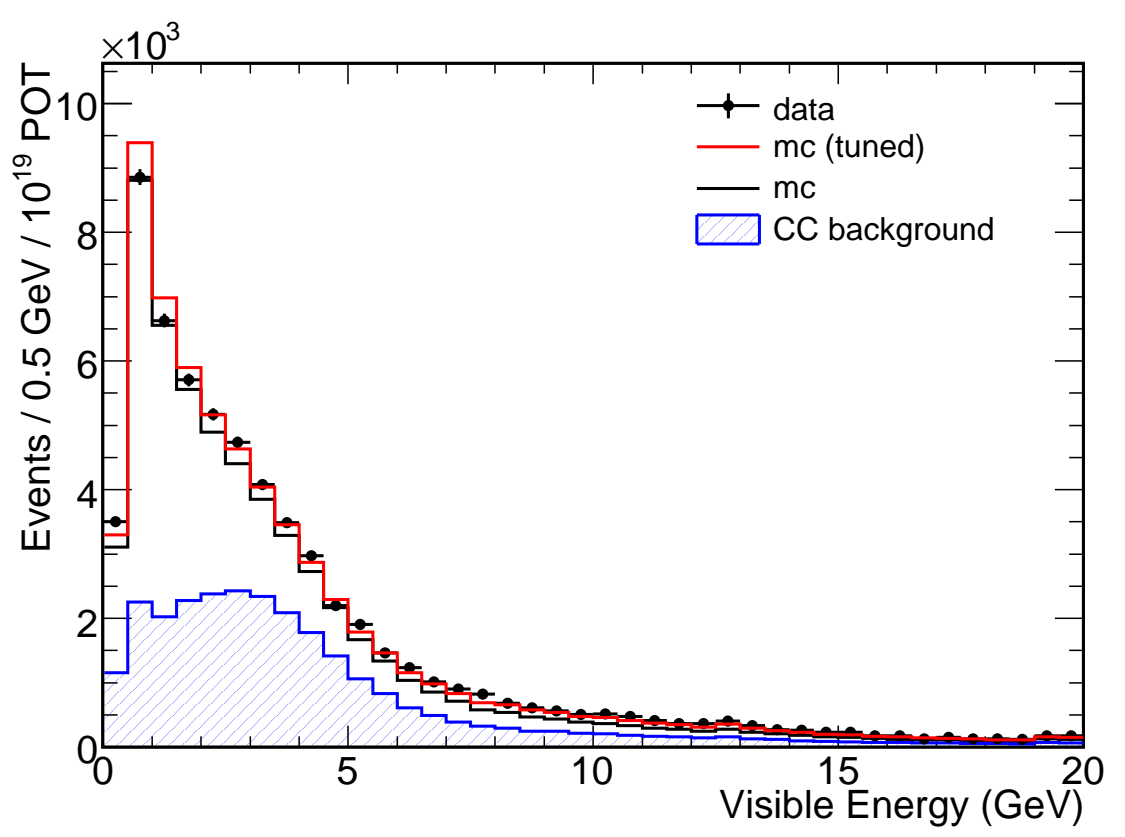

Figure 5.18: Selected neutral current spectrum. This plot shows the energy spectrum of selected neutral current events for data (black markers) and Monte Carlo. The red line corresponds to the tuned Monte Carlo. The blue, hatched distribution shows misidentified charged current events.

\subsection{Artificial Neural Networks}

In this section, we present a neutral current event selection using artificial neural networks. First, the principles of the technique are introduced. Then, we describe how it is implemented in the context of this analysis and present the results.

\subsubsection{Principle of the method}

Artificial neural networks (ANNs) are a widely used technique in the field of machine learning, especially for pattern recognition. They are inspired by the human brain's architecture of interconnected neuron cells and mimic its learning processes. Artificial neural networks classify patterns into a predefined number of classes according to a set of input variables. The main feature of neural networks is the ability to improve their performance by training on a set of model patterns.

The fundamental unit of the neural network is a "neuron". The artificial 
neuron mimics the processes which occur in the synapses of brain cells. Its task is to convert the pattern of incoming activity from other neurons into a single outgoing activity which is subsequently broadcast to other units. In artificial neural networks, this is achieved by means of two quantities: a set of weights and a choice of input-output function. A graphical representation of a neuron (taken from [94]) is shown in Figure 5.19. Mathematically, each neuron j computes a weighted sum of its inputs of the form

$$
a_{j}=\sum_{i} w_{j i} z_{i}+b_{j}
$$

$z_{i}$ are the inputs and $a_{j}$ is called the activation of the neuron $j . w_{i j}$ are the weights corresponding to the inputs $i$ and $b_{j}$ is an overall bias. The activation is transformed by a suitable input-output function to obtain the output $z_{j}$ :

$$
z_{j}=\phi\left(a_{j}\right)
$$

The complete neural network consists of multiple layers of neurons. The "input layer" takes the data on which the classification task is to be performed. In the case of a face recognition problem, for example, this could be the pixel-by-pixel information of a camera image or derived quantities, such as the ratio of height and width of the image. There is one neuron for each input variable.

The "output layer" consists of neurons for the different categories, corresponding to the different possible outcomes of the classification. In the example of the face recognition problem, there would be one neuron for each person to be recognised. The strength of activity in a given output neuron is a measure of confidence in the current input belonging to the corresponding category. Depending on the application, the neuron with the strongest signal is either directly taken to be the correct answer or further restrictions, like a minimum signal strength or a 


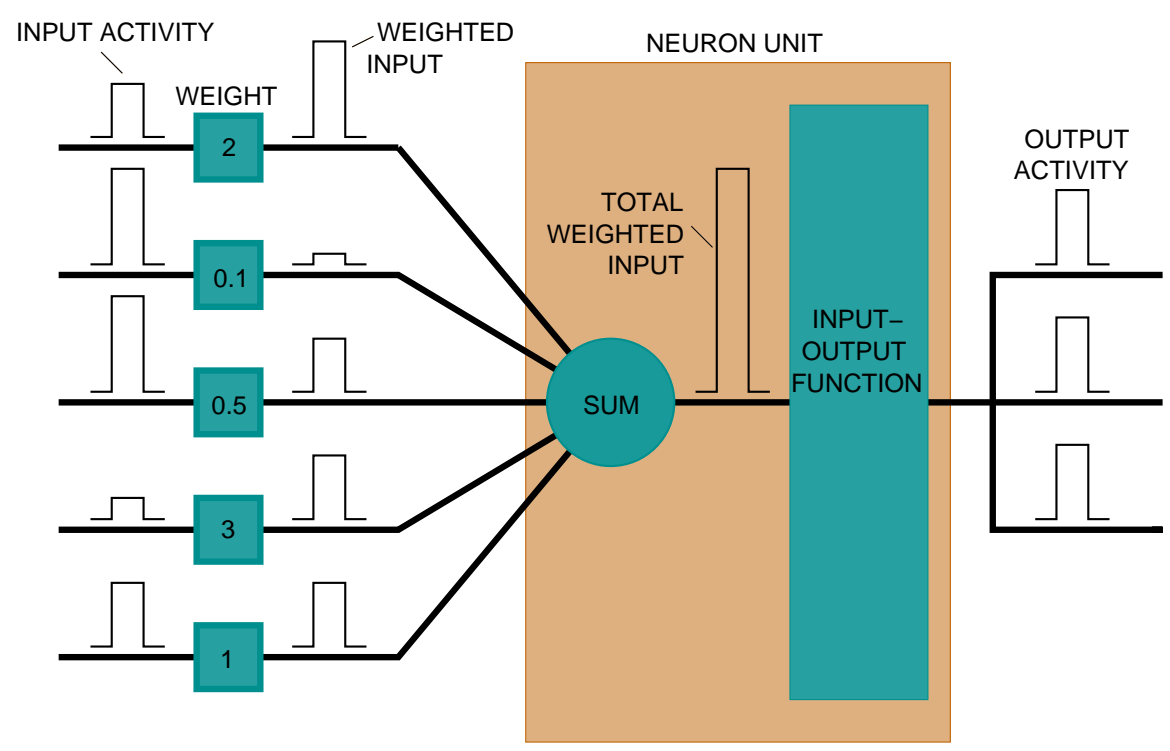

Figure 5.19: Schematic representation of a neuron. Each input is weighted individually before being summed up. The neuron computes the output from the summed weighted input using an input-output function. The output is distributed to the neurons in the next layer. Figure taken from [94].

minimum separation between categories can be imposed.

Between the input and output layers there are a number of hidden layers of interconnected neurons. Most commonly, a single hidden layer of neurons is used. The number of hidden layers and the number of neurons in each one determine the complexity of the function the neural network can represent. More neurons can represent more complex functions of the input variables.

After the layout of the neural network has been chosen, it has to be trained with events whose class is known. In our face recognition example, this would be done with a selection of photographs of the people to be recognised. Training is an iterative process. In each step, the neural network assigns the training events to the different classes. By comparing the assigned class to the true class for all events, an overall classification error for the neural network is calculated. Then the weights of the individual neurons are adjusted and the whole process is repeated. The weights are varied until the classification error reaches a minimum. The more complex the neural network layout, the more training events and training 
cycles are needed. It is however not beneficial to arbitrarily increase the number of layers since the available training data only sparsely populates the resulting multidimensional parameter space. In the machine learning literature, this problem is commonly referred to as the curse of dimensionality [95].

For large, complex neural networks, training is a computing intensive process and the exact algorithm used to find the optimal weight configuration becomes important. The most commonly used algorithm is called "back propagation" [96]. Essentially, one computes the first derivatives of the errors with respect to the weights in the output layer and adjusts the weights in the previous layer. The computation is repeated sequentially for each layer, thus "propagating" the error back through the network.

A potential pitfall for neural network applications is the problem of overtraining. Overtraining occurs when the network "learns" all the available training events too well, getting sensitive to small fluctuations rather then using the general distinguishing features of the input distributions. The neural network fails to generalise and therefore performs worse on an independent test sample. This problem is addressed by comparing the error on an independent test sample to the error on the training sample. Once the error on the test sample doesn't decrease anymore, training is stopped.

A more extensive treatment of the theory of neural networks can be found in [97].

\subsubsection{Implementation of neural networks for a Neutral Cur- rent event selection}

As described in chapter 4, one of the most distinguishing features between neutrino events in MINOS is whether or not a track is found during event reconstruction. Many other potentially useful variables for the discrimination are well defined only 
in cases where a track is present. We therefore decided to construct two separate neural networks, one for the classification of events with tracks and one for events without.

The networks used for this analysis are simple "feed-forward" networks using a sigmoid function as input-output function for hidden layer neurons and a linear function for output neurons. Feed-forward refers to the fact that the output of all neurons goes to the following network layer such that there are no feedback loops. A sigmoid function is defined as follows:

$$
\phi(x)=\frac{1}{1+\mathrm{e}^{-x}}
$$

This type of neural network is called a multilayer perceptron. It is the most commonly used neural networks because of two theorems: Firstly, a linear combination of sigmoid neurons can approximate any continuous function [98]. Secondly, when trained with an output of 1 for signal and 0 for background, the approximated function of inputs $X$ is the probability of the input event being signal, knowing $X$.

Each neuron has set of weights $w_{i}$ corresponding to the inputs $x_{i}$ and a bias $b$. If $\mathrm{X}$ is the vector of inputs $x_{i}$ to a given neuron $j$, the output is:

$$
\phi_{j}(X)=\frac{1}{1+\mathrm{e}^{-\left(\sum_{i} w_{j i} x_{i}+b_{j}\right)}}
$$

The derivative of the sigmoid function has the following advantageous property:

$$
\phi^{\prime}(x)=\phi(x)(1-\phi(x))
$$

Using this relation, it can be shown that the derivatives of the network error with respect to the network weights take the following analytic form (see for example 

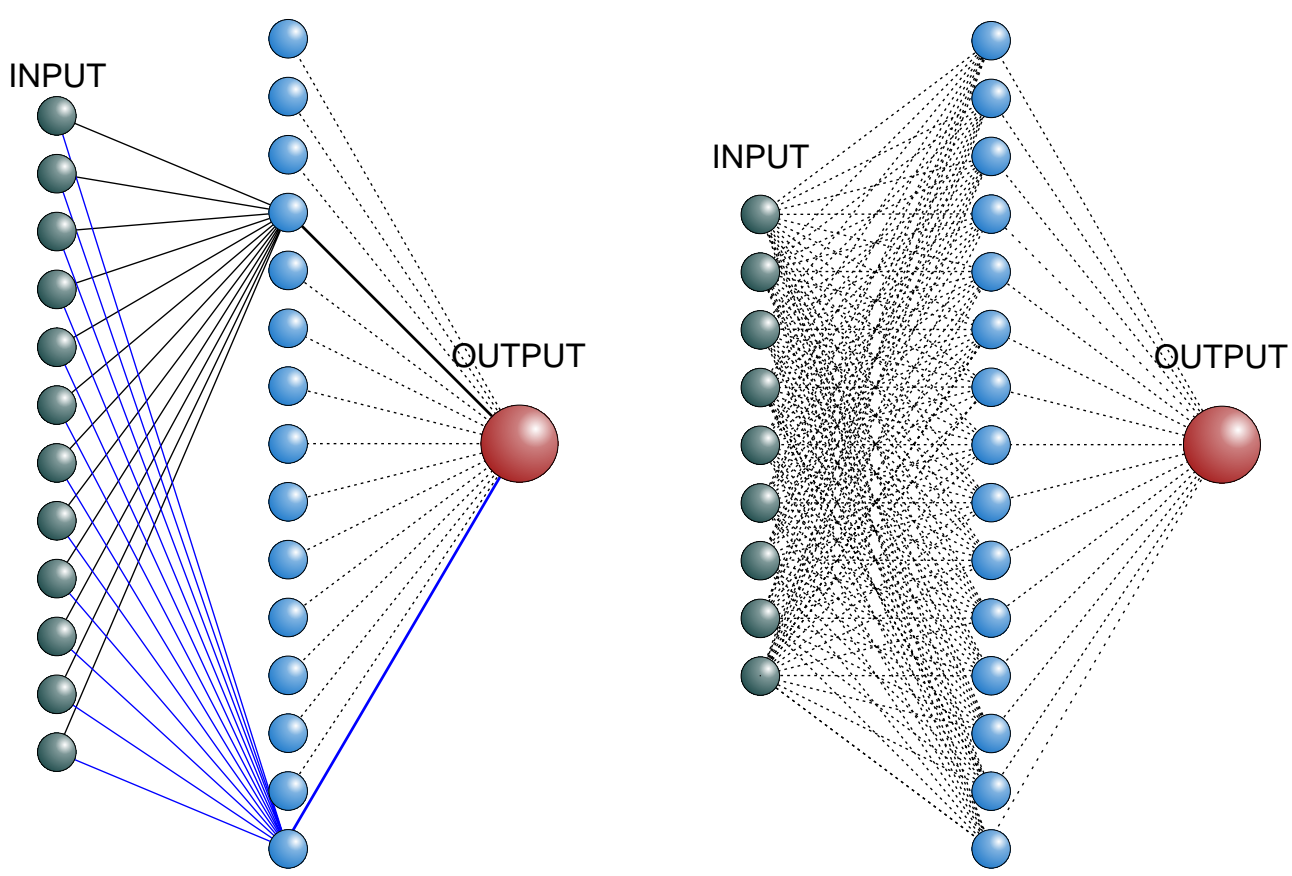

Figure 5.20: Schematic representation of the neural networks used. The left drawing shows the neural network operating on events with tracks, the right drawing the one used for events which only consist of a shower. The track-ANN only shows two combinations of input variables for better clarity. Both networks use 15 sigmoid nodes in one hidden layer.

$[97])$ :

$$
\frac{\partial E^{n}}{\partial w_{j i}}=x_{i} z_{j}\left(1-z_{j}\right) \sum_{k=1}^{c} w_{k j} \delta_{k}
$$

where $E^{n}$ is the total network error for pattern $n$ and the sum runs over all output units. The $x_{i}$ correspond to the inputs for pattern $n$ and $z_{j}$ is the weighted sum of inputs at the node $j$.

Both networks used in this analysis use one hidden layer of neurons, and exactly one neuron in the output layer. High activity in the output neuron is interpreted as charged-current-like and low activity as neutral-current-like. The layout of the two neural networks used is shown in detail in Figure 5.20.

The input variables for the ANNs were chosen by examining Monte Carlo distributions of signal and background after fiducial and pre-selection cuts. Events longer than 40 detector planes were preselected as charged current events. This 
ensures, that the neural network is optimised to provide classification for the more difficult short events.

The most promising quantities in terms of differentiation between signal and background were chosen. They will be discussed in detail in the following section.

\subsubsection{Input variables}

In order to distinguish between neutral current and charged current neutrino interactions, both the topology of hits in the event and the pulse height of individual hits was used. The following nine quantities are used as input to both ANNs:

- eventEnergy $\equiv$ total calorimetric energy in the event

- eventPlanes $\equiv$ total number of planes in the event

- nstrips $\equiv$ total number of strips in the event

- evtPHperPlane $\equiv$ event pulse height per plane

- evtPHperStrip $\equiv$ event pulse height per strip

- shwPHperStrip $\equiv$ shower pulse height per strip

- triPlanePH1 $\equiv$ pulse height in first 3 planes

- triPlanePH2 $\equiv$ pulse height in planes 4-6 (if present)

- triPlaneOverPH $\equiv$ pulse height in planes 7 and above (if present)

The first three variables are global event variables related to the spatial extent and energy of the event. The next set of three variables are ratios of reconstruction quantities. They are related to the energy density and energy profile of the event. The final three variables represent the energy in fixed windows in plane space. These are a measure of the longitudinal shower (and event) development.

In events where a track was reconstructed, the following additional variables are used:

- ntracks $\equiv$ number of reconstructed tracks

- trackExtension $\equiv$ track planes - shower planes 
- trkPHperPlane $\equiv$ track pulse height per plane

These additional variables are used to make a decision on how "muon-like" the reconstructed track in an event is. If there is more than one track found in the event, it is more likely that one is a muon track. The trackExtension variable has been explained in detail for the simple, cut-based selection (see section 5.2). The trkPHperPlane variable is related to $\frac{d E}{d x}$ and therefore useful to distinguish between muon tracks and tracks reconstructed from shower hits. In case multiple tracks are reconstructed in an event, the two variables trackExtension and trkPHperPlane are defined for the longest one.

Figure 5.21 and Figure 5.22 show Monte Carlo and data distributions for all input variables. In general, the agreement between data and Monte Carlo simulation is good. Some discrepancies are observed in variables relating to the exact shape and evolution of the hadronic shower. This is a well known deficiency of the MINOS Monte Carlo simulation which will be addressed in the next release of the software.

\subsubsection{Performance of the ANN}

The two artificial neural networks were trained using a Monte Carlo sample corresponding to an exposure of $4.4 \times 10^{18}$ protons-on-target, using a Broyden, Fletcher, Goldfarb, Shanno (BFGS) [99] implementation of the back propagation algorithm. The sample was split into a training and a validation set. The evolution of the neural network classification error during training is shown in Figure 5.23 for the track network (left) and the non-track network (right). Training was stopped after 100 epochs, where the classification error on the independent validation set has levelled off sufficiently. An epoch refers to the usage of all input events exactly once.

Figure 5.24 shows the distribution of ANN output values for events with a 

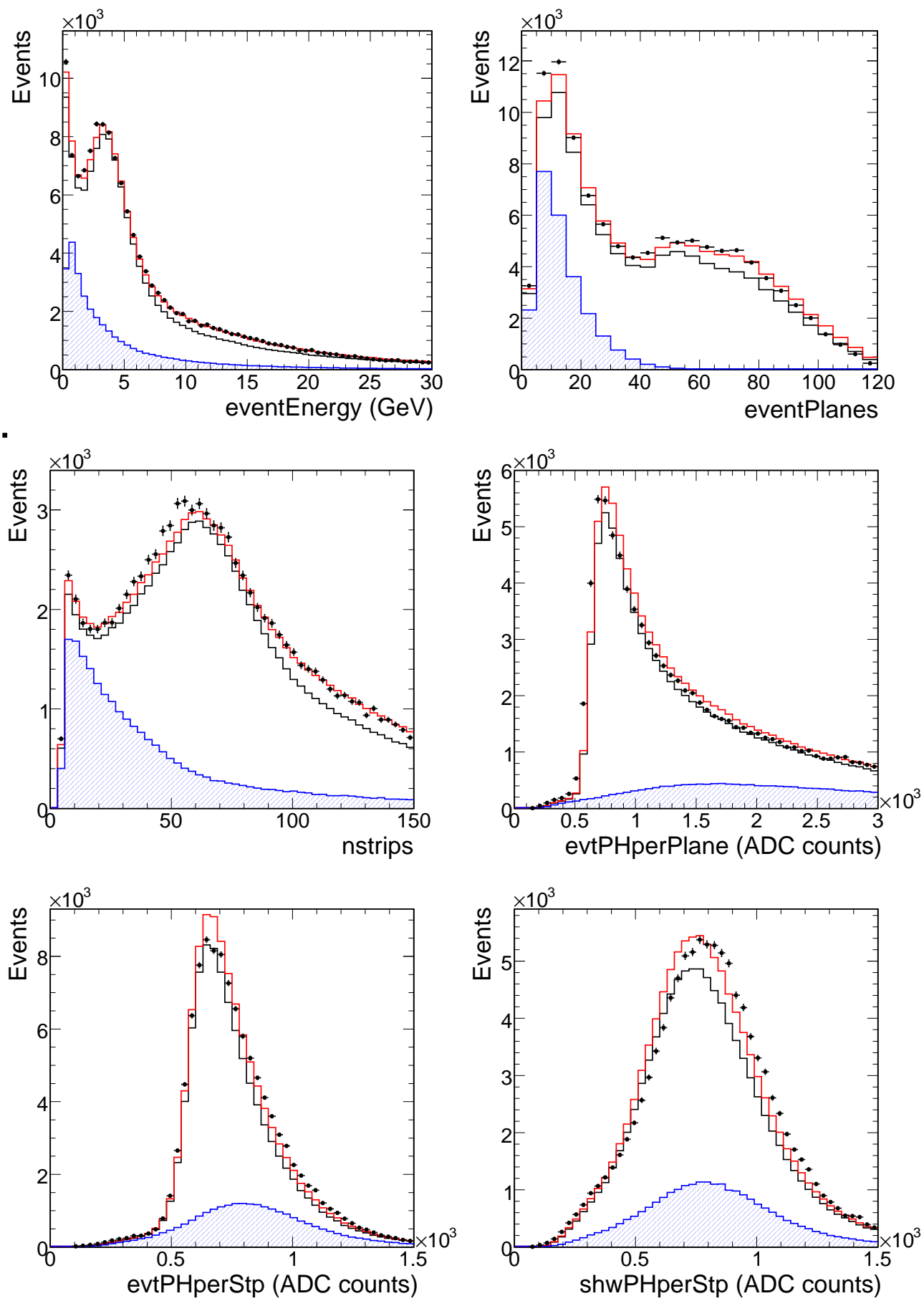

Figure 5.21: ANN input variables. The red line shows the Monte Carlo simulation after tuning the hadron production model, the markers show the data. The neutral current signal is shown as blue, hatched distributions. The untuned Monte Carlo is shown as a black line for comparison. 

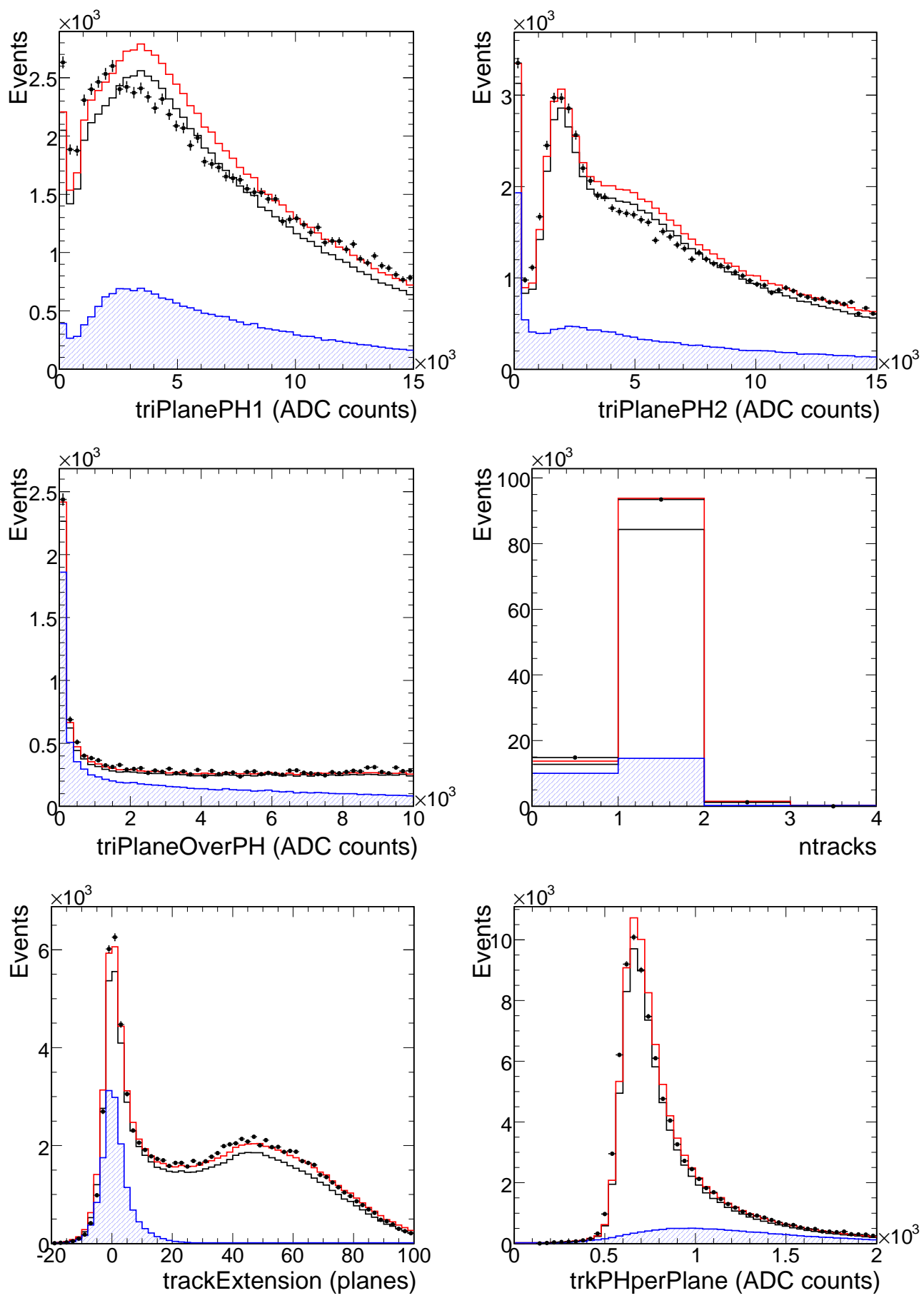

Figure 5.22: ANN input variables (cont'd). The red line shows the Monte Carlo simulation after tuning the hadron production model, the markers show the data. The neutral current signal is shown as blue, hatched distributions. The untuned Monte Carlo is shown as a black line for comparison. 

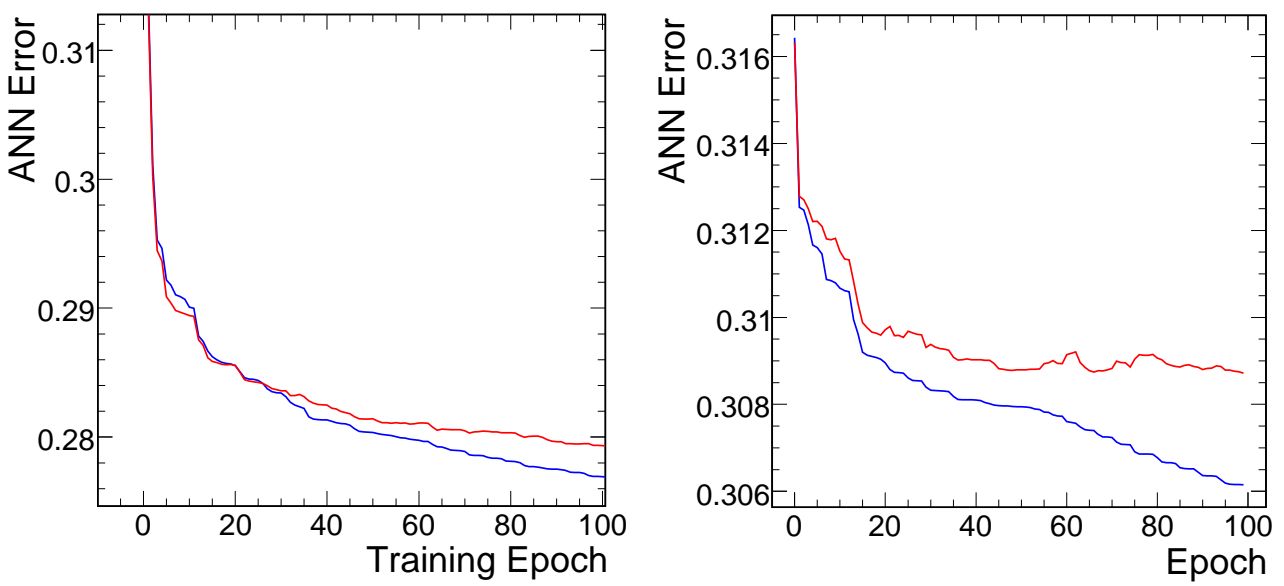

Figure 5.23: Neural network training for the track ANN(left) and the no-track ANN (right). The classification error on the training sample, shown in blue, decreases monotonically with continued training, whereas the error on the independent test sample (shown in red) levels off.

reconstructed track (left) and events without a track (right). The Monte Carlo set used for these performance plots is independent from the training and validation sets.

The output distributions for events with tracks shows a clear separation between neutral and charged current events. The true neutral current interactions contribute to a peak at around 0.5. A second peak at 1.0 is entirely made up of charged current events. The fact that there is a constant fraction of charged current events in the neutral current signal peak shows that some classes of events are indistinguishable for the neural network. There is however a class of events which the neural network clearly identifies as charged currents and which can therefore be removed from the sample. The exact position of the cut separating signal from background is a trade-off between selection efficiency and purity of the selected sample. For the purpose of this analysis, a cut at 0.7 is used.

The output distribution for events with no reconstructed track shows far less separation between signal and background. Below an output value of about 0.2 , the neutral current component is enriched in the sample. For the rest of the distri- 

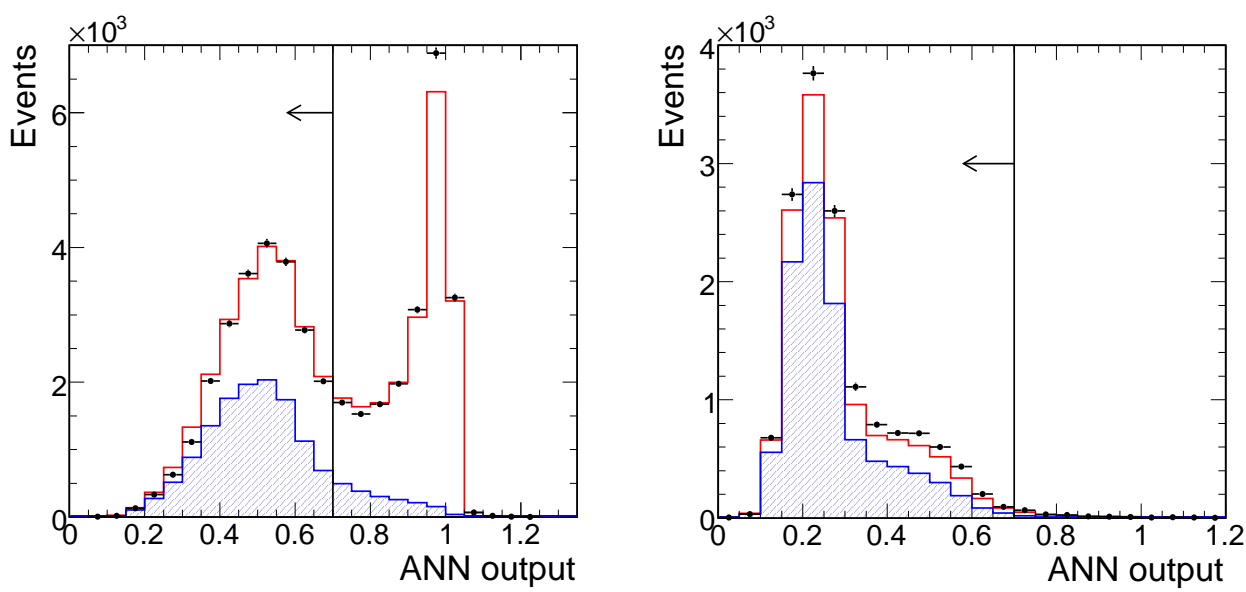

Figure 5.24: ANN output distributions. The red line shows the ANN output values for Monte Carlo for events with tracks (left) and without tracks (right); the black markers show the Near Detector data. The neutral current signal is shown in blue.

bution there is no further separation, meaning the input variables lack separation power. For the purpose of this analysis it was decided to opt for a higher efficiency and therefore keep all events below 0.7. This cuts just a few obvious charged current events while maintaining a high selection efficiency. It is however possible to achieve a higher purity selection by making a harsher cut, for example at 0.2.

The selected neutral current energy spectrum using the ANN method is shown in Figure 5.25. It looks similar to Figure 5.18 but with a smaller background contribution in the range of $2-5 \mathrm{GeV}$.

The overall efficiency for the ANN selection method, averaged over all energies, is $90 \%$, with a sample purity of $59 \%$. The statistical uncertainty on these numbers is $\sim 0.5 \%$.

Efficiency and purity distributions as a function of energy for this selection are shown in comparison with the simple cut-based method in the following section. 


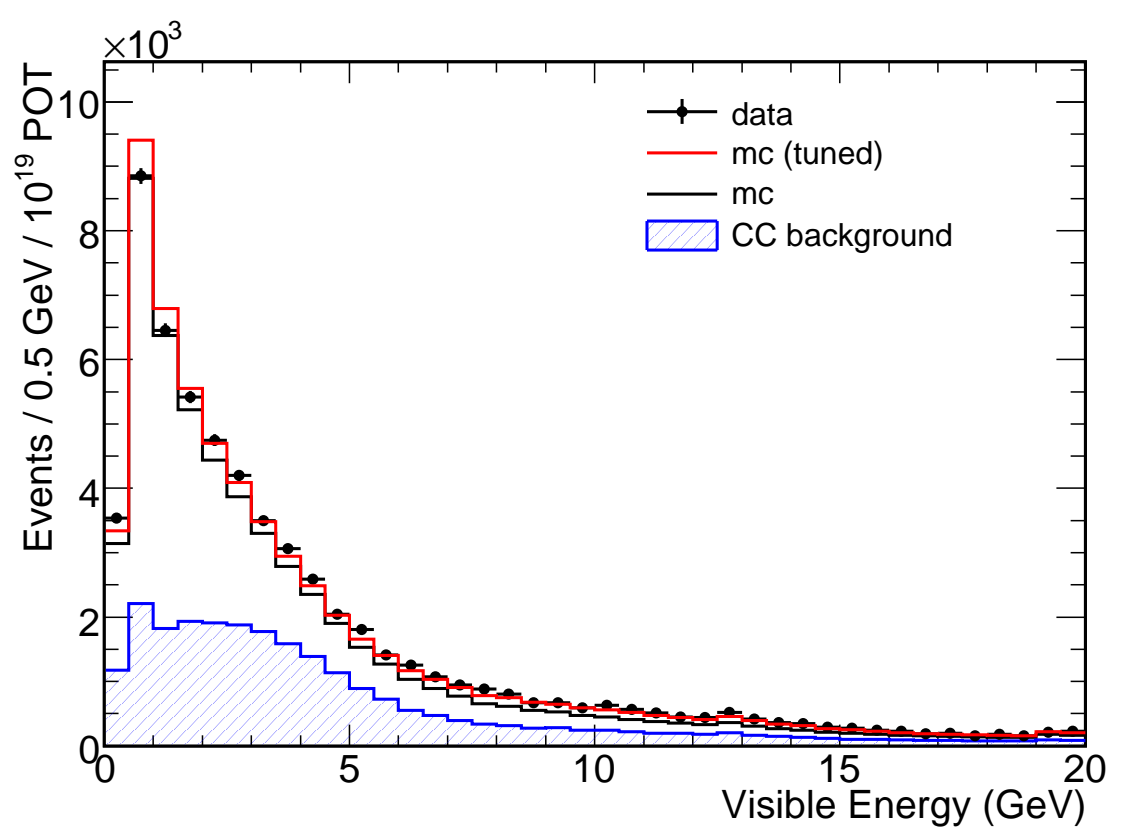

Figure 5.25: ANN selected neutral current spectrum. This plot shows the energy spectrum of selected neutral current events for data (black markers) and Monte Carlo, using the ANN method. This is the corresponding plot to Figure 5.18

\subsection{Results}

Figure 5.26 shows a comparison of selection efficiency and purity for the two different selections detailed in sections 5.2 and 5.3. The results of the simple, cut-based selection are shown in red, the results of the artificial neural network selection in blue.

The advantages of the more complicated method are visible in both efficiency and purity. The efficiency distribution as a function of visible energy is closer to flat for the ANN selection, whereas it has a considerable slope in the cut selection.

The purity for the ANN selection is higher, most noticeably in the region where the CC spectrum peaks. The improvements are on the order of $10 \%$ rather than factors of two or three, but this is expected. It has been experienced by many MINOS collaborators investigating neutral current selection methods that there is a limit to what can be achieved in the case of high momentum-transfer charged 

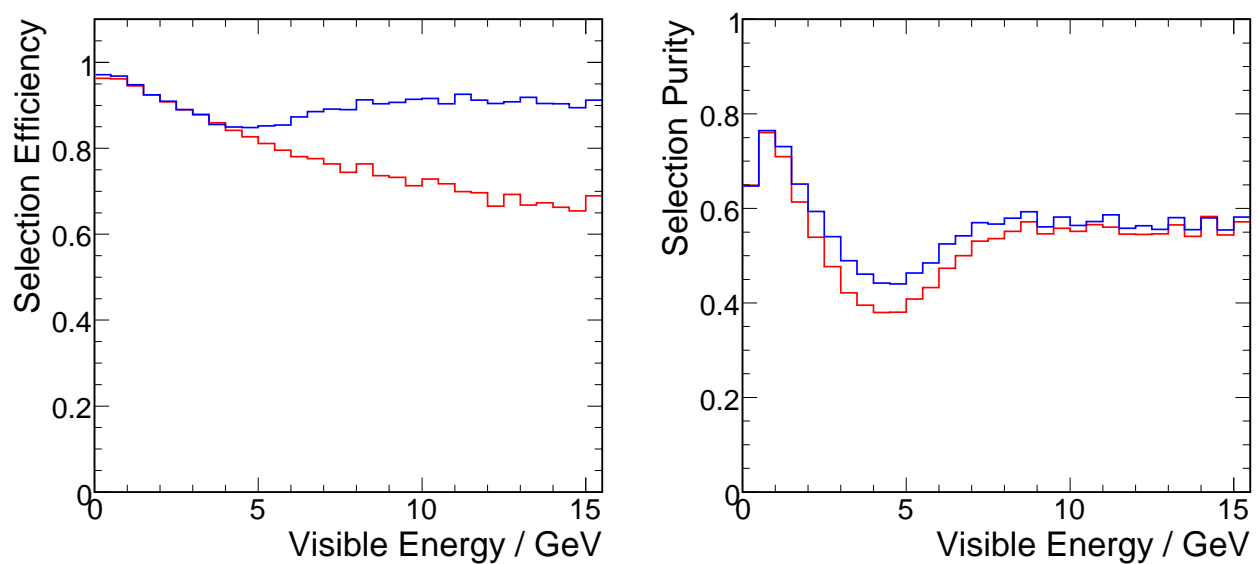

Figure 5.26: Comparison of selection efficiency and purity. The left figure shows the efficiency for selecting neutral current events using the cut method (red) and the ANN method (blue). The right figure shows the same comparison for the purity of the selected sample. In both cases, the ANN performs better than the simple, cut-based selection.

current events. To say this in another way: Some CC events look just like neutral currents.

\subsection{Conclusion}

A procedure to select neutral current neutrino interaction in the MINOS Near Detector has been developed. The selection proceeds in two distinct steps, a pre-selection stage which improves data quality by removing badly reconstructed events from the data sample, and a neutral current selection stage which removed charged current events. For the second stage, both a simple cut-based selection and a method using artificial neutral networks (ANNs) have been described. The ANN technique is shown to perform better than the simple method.

In the next chapter, the ANN selection is used to extract cross-section parameters from the Near Detector data. 


\section{Chapter 6}

\section{NC Cross-section Measurement in the Near Detector}

This chapter describes the final part of the analysis of neutral current neutrino interactions presented in this thesis. The goal of the measurement is to extract information about the cross-section for neutral current neutrino interactions from the reconstructed energy spectrum of selected neutral current events in the Near Detector.

Improved knowledge of the neutral current interaction cross-section is interesting for two reasons: Firstly, neutrino cross-sections at the energies relevant in MINOS are poorly constrained. The only available measurements in the subto-few $\mathrm{GeV}$ range come from the bubble chamber experiments at Argonne and Brookhaven National Laboratories [100, 101], using charged current interactions. They have uncertainties of the order of 10-20\%. Furthermore, there is even less data on neutral current interaction cross-sections. The only measurement in the relevant energy range comes from the Gargamelle experiment at CERN [102]. An improved measurement of these cross-sections is therefore an interesting result in its own right. Secondly, the improved cross-section data can be used to improve the Monte Carlo prediction of the neutral current energy spectrum at the Far De- 
tector. This will help to reduce systematic errors for a future search for oscillations into sterile neutrinos.

\subsection{Overview of the analysis procedure}

The starting point of this analysis is a neutral current reconstructed energy spectrum in data and Monte Carlo. Neutral current events are selected using the neural network technique described in detail in chapter 5. The Monte Carlo is tuned for hadron production in the NuMI target. Essentially, the parent hadrons, which decay to produce neutrinos, are weighted up or down as a function of transverse and longitudinal momentum, in order to improve the data/Monte Carlo agreement. This Monte Carlo tuning, described in more detail in chapter 7, uses fits to the reconstructed energy spectrum of charged current events. Therefore, this technique effectively adjusts the product of beam flux and charged current crosssections. The only remaining free parameter is the neutral current cross-section as a function of true neutrino energy.

Extracting cross-section information for neutral current interactions is not straightforward, since the energy of the incident neutrino is not measured in the detector. Only the energy transferred to the nucleus which was hit is measured in a hadronic shower. This energy can be anywhere between zero and the energy of the incident neutrino. The number of events in a given bin $i$ of reconstructed energy $E_{\text {reco }}^{i}$ is therefore influenced by the cross-section at all energies $E_{\text {true }} \gtrsim E_{\text {reco }}^{i}$. In order to distinguish between these two energies, we always refer to the neutrino energy as "true neutrino energy" or $E_{\text {true }}$ and call the quantity measured in the detector "reconstructed energy" or $E_{\text {reco }}$.

To break the degeneracy between cross-sections at different true neutrino energies, two steps are taken: First, the cross-sections are only fitted in terms of effective scale factors in four fairly wide bins in true neutrino energy. Secondly, 


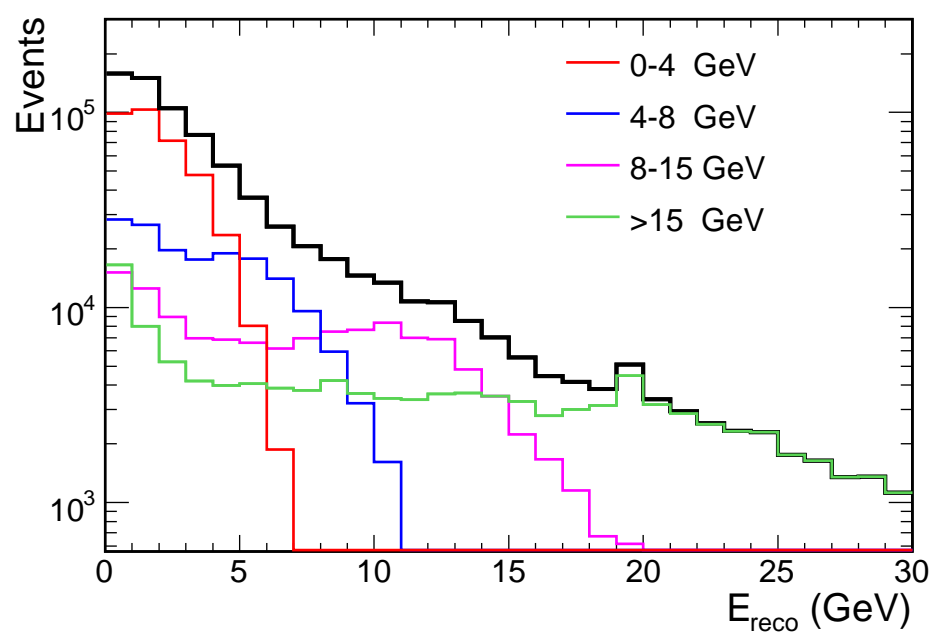

Figure 6.1: Neutral current spectrum contributions from different true neutrino energy ranges. Neutrinos of a given true energy $E_{\text {true }}$ contribute to all energies $E_{\text {reco }}<E_{\text {true }}$ in reconstructed energy. The black line shows the sum of the four coloured contributions.

data and Monte Carlo for three different beam configurations are used. These beam configurations differ mainly in the position of the peak in the neutrino energy spectrum.

The neutral current reconstructed energy distribution is fitted by scaling the number of events in four ranges of true neutrino energy. These scale factors can be interpreted as effective neutral current cross-sections for these energy ranges. The ranges are $0-4 \mathrm{GeV}, 4-8 \mathrm{GeV}, 8-15 \mathrm{GeV}$ and $>15 \mathrm{GeV}$. The first three ranges approximately cover the peak in neutrino flux in the three different beam configurations and the last bin covers the high-energy tail. Figure 6.1 shows how the low energy beam Monte Carlo spectrum is composed from contributions from these three ranges in true neutrino energy. 


\subsection{Data samples}

This analysis uses the three beam configurations L010/185kA, L100/200kA and L250/200kA, as given in Table 3.1 on page 56. For the sake of readability, they will be referred to as low energy, medium energy and high energy in the remainder of this chapter.

As shown in Table 3.1, the recorded exposure in the different beam configuration varies significantly. It is $1.27 \times 10^{20}$ protons-on-target $(\mathrm{POT})$ in the low energy, $1.11 \times 10^{20}$ POT in the medium energy and $1.55 \times 10^{18}$ POT in the high energy beam. This is the complete first data set, up to the 2006 shutdown. Furthermore, the available Monte Carlo statistics in these configurations is different as well. It was decided to scale the different beam configurations to the same integral number of events in order to give them comparable weights in the cross-section fit and therefore obtain similar accuracies in the various fit ranges.

Figure 6.2 shows the neutral current reconstructed energy spectra in data and Monte Carlo for the three different beam configurations. These are the input distributions for the cross-section fit.

\subsection{The fitting technique}

The extraction of cross-section scale factors proceeds in a similar fashion to the fit described in the Mock Data Challenge chapter 4. The selected neutral current reconstructed energy spectra in the three different beam configurations are fit simultaneously, using a binned, extended maximum likelihood method. The likelihood function $\mathcal{L}$ is given in equation 6.1.

$$
\mathcal{L}=\frac{e^{-N} N^{D}}{D !} \prod_{i}\left(\frac{n_{i}}{N}\right)^{d_{i}} \quad \text { with }\left\{\begin{array}{l}
d_{i}:=\text { data events in bin } i \\
n_{i}:=\text { MC events in bin } i \\
D:=\sum_{i} d_{i}, N:=\sum_{i} n_{i}
\end{array}\right.
$$




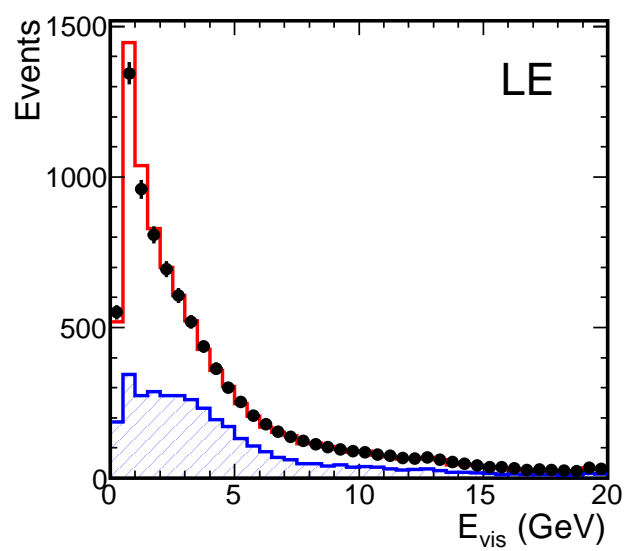

\section{+ Data \\ Monte Carlo \\ CC background}
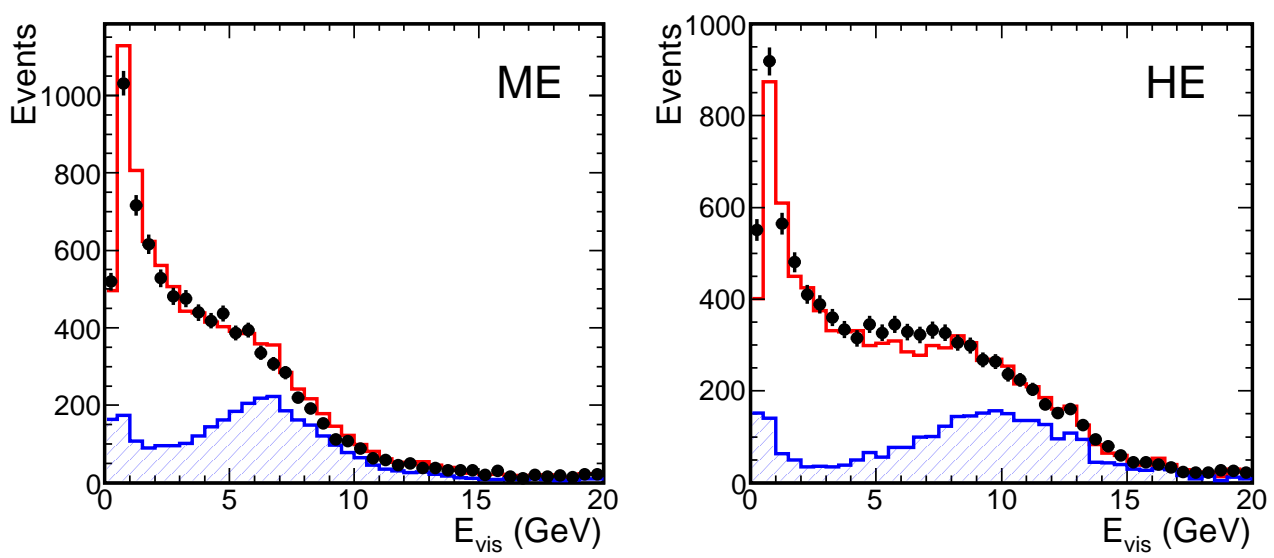

Figure 6.2: Neutral current reconstructed energy spectra for the three different beams configurations. Data is shown as black markers with error bars, the tuned Monte Carlo is shown in red. The blue, hatched histogram shows the wrongly selected charged current background. 
The bin index $i$ runs over the reconstructed energy spectra of all three beam configurations. The fit returns four scale factors representing corrections to the neutral current cross-sections included in the NEUGEN Monte Carlo simulation. The best-fit values for these cross-section parameters are obtained by minimising the negative logarithm of the likelihood $\mathcal{L}$ using MINUIT.

\subsubsection{Verifying the fit}

Multiple tests have been performed in order to verify that the fitting software performs as expected. First, a mock data set was created by scaling the MINOS Monte Carlo with four known scale factors in the previously mentioned ranges in true neutrino energy. This mock data set contained exactly the same statistical fluctuations as the Monte Carlo used in the fit. Consequently the fit should return the input parameters within the machine precision used. This exercise was performed 500 times for different scale parameter values. The fit returned the correct scale parameters to within floating point precision for every single experiment.

In order to simulate a more realistic scenario, another mock data set was created by first scaling the neutral current component by four scale factors as above and then fluctuating the number of events in each bin according to a Poisson probability. This results in a statistically independent mock data set. The fit results should now vary around the input value with a spread consistent with statistical fluctuations. This consistency was checked using "pull distributions". The pull value $\mathrm{p}$ is defined as:

$$
p=\frac{f_{0}-f}{\sigma} \quad \text { with } \quad\left\{\begin{array}{l}
f_{0}:=\text { input value } \\
f:=\text { fit result } \\
\sigma:=\text { fit error }
\end{array}\right.
$$

If the fit is unbiased and the fit errors are estimated correctly, the pull values for a large number of experiments are expected to follow a standard normal distribution 
$N(0,1)$. Figure 6.3 shows pull distributions for the four scale parameters for $\sim 850$ mock experiments. The pull values follow the expected distribution in all four cases. There is no significant bias and the width of the distributions is consistent with a value of 1 , indicating correct error estimates.

These results give confidence that the fitting procedure is working properly and consequently it is applied to the measured Near Detector data in the following section.

\subsection{NC cross-section results}

The combined fit to the data in all three beam configurations yields the following best-fit values for the neutral current cross-section scale parameters

$$
\begin{array}{ll}
\text { Par } 1(0-4 \mathrm{GeV}): & 0.881 \pm 0.035 \\
\text { Par } 2(4-8 \mathrm{GeV}): & 0.967 \pm 0.026 \\
\text { Par } 3(8-15 \mathrm{GeV}): & 1.101 \pm 0.033 \\
\text { Par } 4(>15 \mathrm{GeV}): & 1.029 \pm 0.041
\end{array}
$$

where the errors given are statistical only. The statistical errors are a combination of the statistical errors on the data and the Monte Carlo.

Due to a lack of Monte Carlo data, the error in the low energy and the high energy beam configurations is dominated by the Monte Carlo statistics. Since the likelihood function does not account for statistical errors on the Monte Carlo, we scale the data down to the Monte Carlo statistics. This results in error bars on the data which actually reflect the Monte Carlo statistics. The actual statistical error on the data can be neglected in comparison. This trick is not used for the medium energy configuration which is dominated by the statistical error on the data.

As expected, the four fit parameters are not completely uncorrelated. Typically, the scale factors in adjacent ranges of true neutrino energy are anti-correlated 

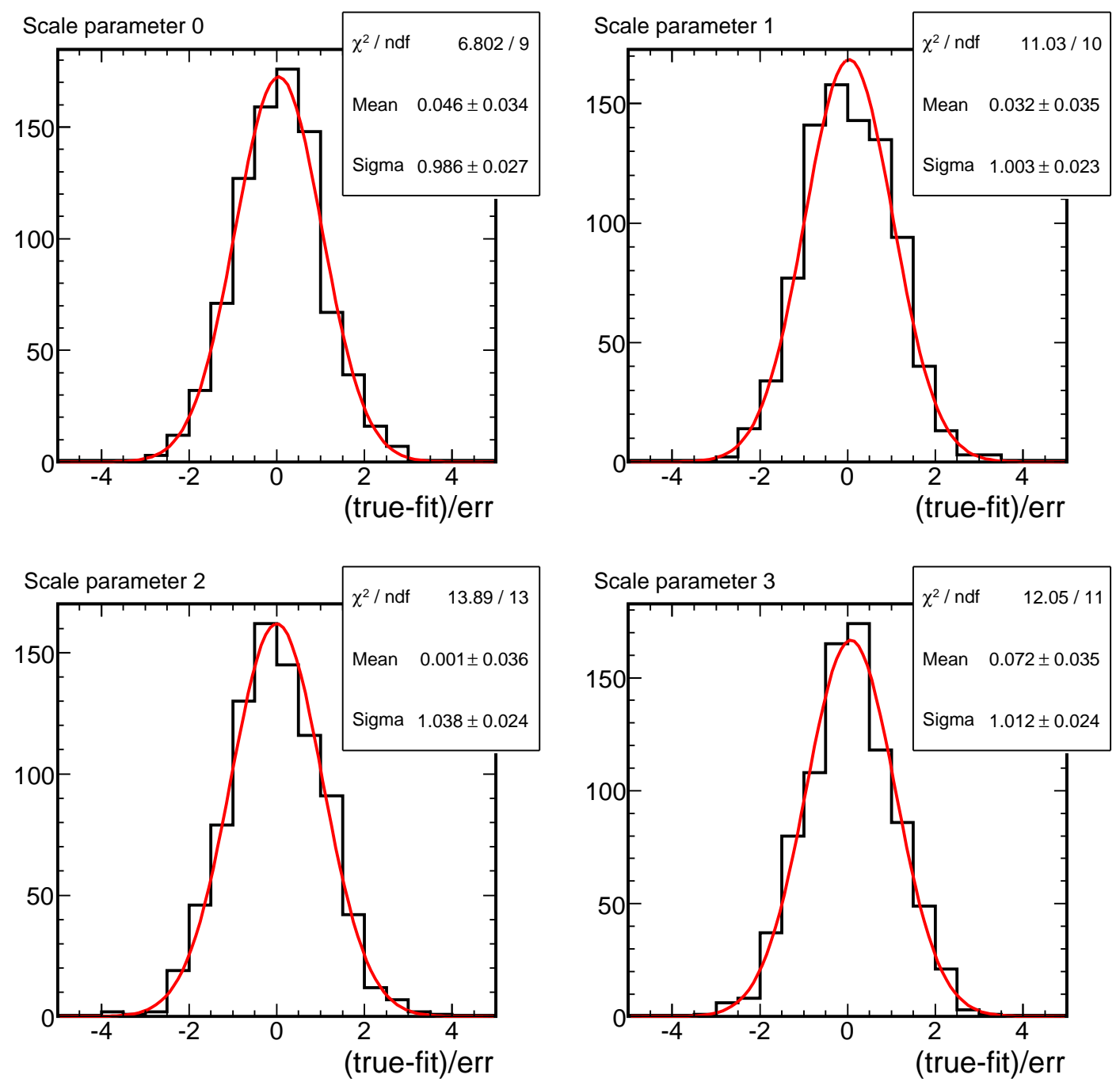

Figure 6.3: Pull distributions using $\sim 850$ mock experiments. There is no significant bias in any of the four fit parameters and the width of the distributions is consistent with 1 . 

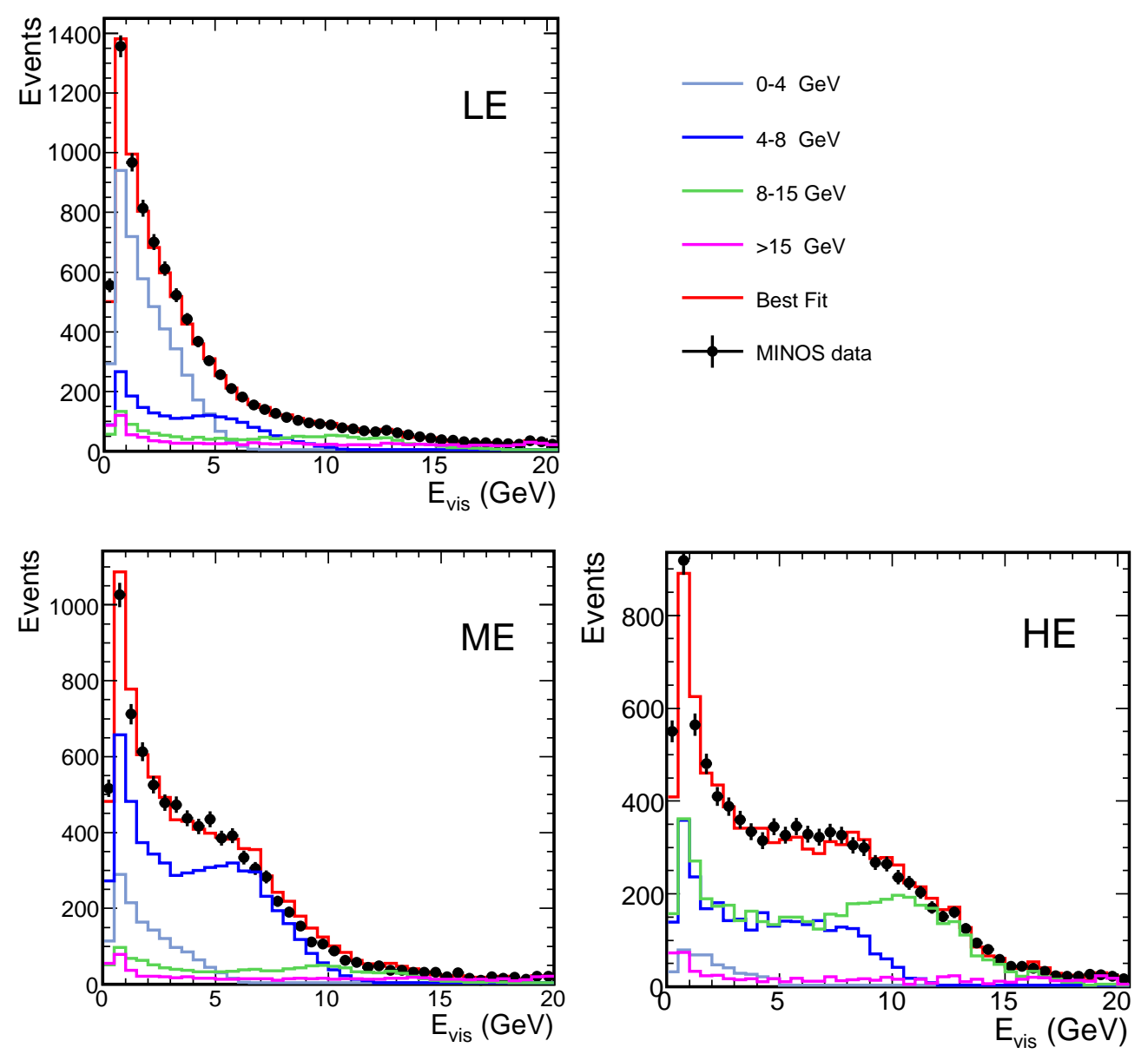

Figure 6.4: Best-fit reconstructed energy distributions for the three beam configurations. Data is shown as black markers and the best-fit Monte Carlo is shown in red. Contributions from the four true neutrino energy ranges are shown in the other colours.

with correlation coefficients ranging from $10 \%$ to $50 \%$.

Figure 6.4 shows the data and best-fit Monte Carlo reconstructed energy spectra for the three beam configurations. The contributions from the four true neutrino energy ranges to the total spectrum are also shown.

The error on the measured cross-section parameters is however expected to be dominated by systematic uncertainties, since the number of neutrino interactions recorded in the Near Detector is sufficiently large. 


\subsection{Systematic error evaluation}

The systematic uncertainties for the measurement of neutral current cross-sections are evaluated by fitting the measured data with systematically modified Monte Carlo distributions and recording the shift in the resulting best-fit parameters. The various systematic effects which were identified are evaluated one at a time. The total systematic error is then computed by taking the quadratic sum of the individual shifts. This procedure is valid if the different systematic effects are assumed to be independent of each other, which should be true in most cases. In the following section 6.5.1, a detailed discussion of which systematic errors are considered is given.

In addition, it is important to point out that each detected shift, however small, is significant since the same data and Monte Carlo events are used in each fit. Fluctuations due to limited Monte Carlo statistics do not affect the result because each systematically modified Monte Carlo set contains the same fluctuations. This way, statistical and systematic uncertainties can be treated independently.

The systematic uncertainties for the measurement of neutral current crosssections in the MINOS Near Detector can be grouped in three categories. They are: beam modelling, interaction modelling and detector/reconstruction effects.

The nature and magnitude of the systematic uncertainties considered in this chapter are motivated by the charged current neutrino disappearance analysis described in chapter 7 . The work on the charged current analysis was completed earlier than the analysis in this chapter. However, for the sake of readability, we chose to conclude the presentation of neutral current work before describing the charged current analysis separately. 


\subsubsection{Effects considered}

Beam modelling As mentioned earlier, the beam modelling uncertainty stems from the uncertainties on the hadron production in the NuMI target and beamline geometry. It is significantly reduced by a hadron production tuning procedure, described in chapter 7. As well as improving the data/Monte Carlo agreement by tuning hadron production parameters, this procedure returns an error envelope for the tuned Monte Carlo simulation. The remaining systematic uncertainty on beam modelling after tuning is evaluated by adding or subtracting the $1 \sigma$ error envelope to the Monte Carlo distribution used in the fit.

Interaction modelling The goal of this analysis is an improved knowledge of neutral current cross-sections in ranges of neutrino energy. A large part of the interaction modelling, namely the cross-sections, therefore constitutes the result of this analysis and does not contribute to the systematic error evaluation.

There is however an additional effect known as intra-nuclear rescattering [103]. In neutrino-nucleon interactions below a few $\mathrm{GeV}$, pions in the final state can reinteract with other nucleons in the same nucleus before emerging. This leads to a distortion of the reconstructed energy spectrum of the hadronic shower. The magnitude of the effect was estimated using two extreme configurations (no pion absorption and full pion absorption) in the Monte Carlo simulation [104]. In this analysis, an offset of $\pm 150 \mathrm{MeV}$ to the reconstructed shower energy was used to simulate this effect.

Detector/Reconstruction effects This category comprises all remaining systematic uncertainties originating in reconstruction and event selection effects, normalisation uncertainties and detector calibration.

Different reconstruction efficiencies for data and Monte Carlo, as well as shape differences in the distributions used to select signal events can result in a system- 

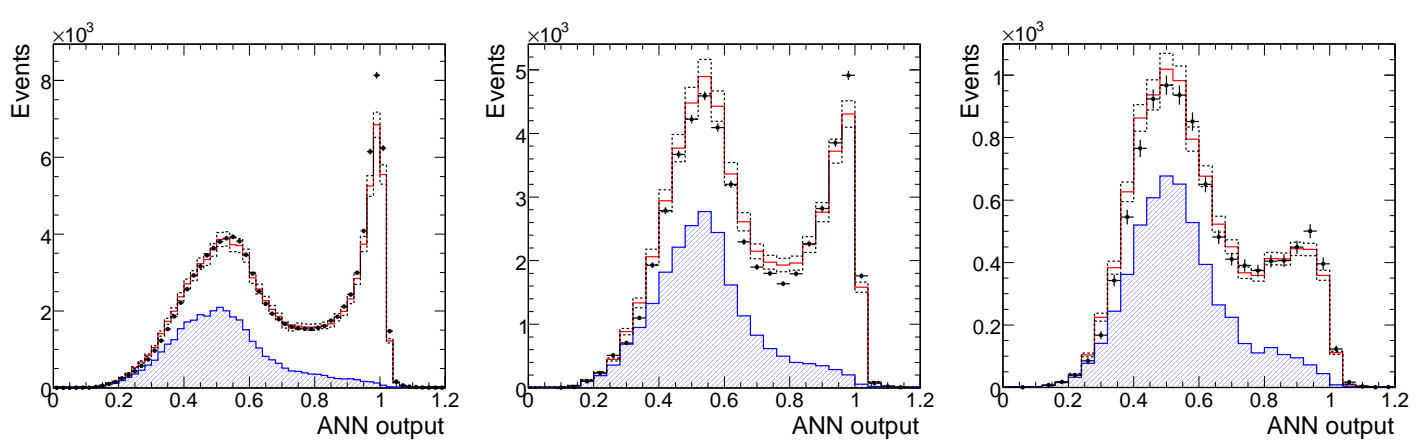

Figure 6.5: ANN output distributions for the three beam configurations (left:LE, middle: ME, right: HE). Data is shown as black markers and the best-fit Monte Carlo is shown in red. The neutral current signal is shown in shaded blue and the dashed lines show the $\pm 1 \sigma$ error bands from the beam flux tuning.

atic uncertainty on the measured cross-sections. This systematic effect is evaluated by changing the position of the selection cut in the ANN output in the Monte Carlo relative to the data. The size for this shift was determined using the level of disagreement in the ANN output distributions shown in Figure 6.5. This disagreement is of course partly due to the cross-section differences we wish to measure. We therefore use the disagreement between data and Monte Carlo after the fit to the data to assess this systematic. A shift of 0.01 in the selection cut position was found to cover the disagreement in all three beam configurations. Having found a reasonable size for this systematic, this shift was applied symmetrically in both directions.

In chapter 5 , section 5.1, we describe a pre-selection method designed to eliminate badly reconstructed events from the data sample. In one of the selection variables, the number of strips in the event, there is a noticeable disagreement between data and Monte Carlo. We therefore decided to evaluate this as an additional systematic uncertainty by varying the cut position in Monte Carlo by \pm 1 strip. Since this is an overestimate of the data/Monte Carlo disagreement, we take $50 \%$ of this as the systematic uncertainty due to the pre-selection.

The systematic uncertainty on the shower energy scale due to detector calibra- 


\begin{tabular}{l|c|c|c|c|c}
\hline Systematic effect & \pm & $\Delta($ Par 1$)$ & $\Delta($ Par 2$)$ & $\Delta($ Par3 $)$ & $\Delta($ Par 4$)$ \\
\hline \hline Beam tuning & + & -0.057 & -0.059 & -0.079 & -0.096 \\
$\pm 1 \sigma$ & - & +0.062 & +0.067 & +0.087 & +0.108 \\
\hline Shower energy & + & +0.079 & +0.068 & +0.009 & -0.239 \\
$\pm 5.6 \%$ & - & -0.086 & -0.068 & -0.017 & +0.264 \\
\hline Intranucl. rescatter. & + & +0.055 & -0.028 & -0.022 & -0.002 \\
$\pm 150 \mathrm{MeV}$ & - & -0.108 & +0.011 & +0.039 & +0.032 \\
\hline Normalisation & + & -0.050 & -0.043 & -0.065 & -0.082 \\
$\pm 4 \%$ & - & +0.054 & +0.047 & +0.071 & +0.089 \\
\hline Strip cut & + & +0.050 & +0.005 & +0.006 & +0.002 \\
$50 \%$ of \pm 1 strip & - & -0.065 & -0.009 & -0.007 & -0.009 \\
\hline ANN cut & + & +0.006 & -0.020 & -0.026 & -0.027 \\
\pm 0.01 & - & -0.006 & +0.021 & +0.029 & +0.025 \\
\hline
\end{tabular}

Table 6.1: Systematic error evaluation. Each entry in this table shows the change in a best-fit cross-section parameter caused by a given systematic error. The systematic uncertainty at low energies gets contributions from intra-nuclear rescattering, the pre-selection minimum strip cut and the shower energy uncertainty. At high energies the error is dominated by the shower energy scale uncertainty.

tion is estimated as $5.6 \%$ (see next chapter).

Finally, an overall normalisation uncertainty of $\pm 4 \%$ is assumed due to POT counting, fiducial detector mass uncertainty and other reconstruction efficiencies.

\subsubsection{Results}

Figures 6.6, 6.7 and 6.8 show the effect of the various systematic shifts on the Near Detector neutral current energy spectrum for the three different beam conditions respectively. The obvious trends are clearly visible: The shower energy scale error for example shifts the distribution to the left/right, whereas the minimum strip cut variation only affects the lowest energy bins.

The effect of each individual systematic shift on the best-fit values is given in Table 6.1. The results are given as deviations from the best-fit value using the tuned MINOS Monte Carlo simulation. The \pm signs distinguish between the $\pm 1 \sigma$ variations on a given systematic parameter. As expected, intra-nuclear rescattering and the minimum strip cut in the pre-selection only have an impact 

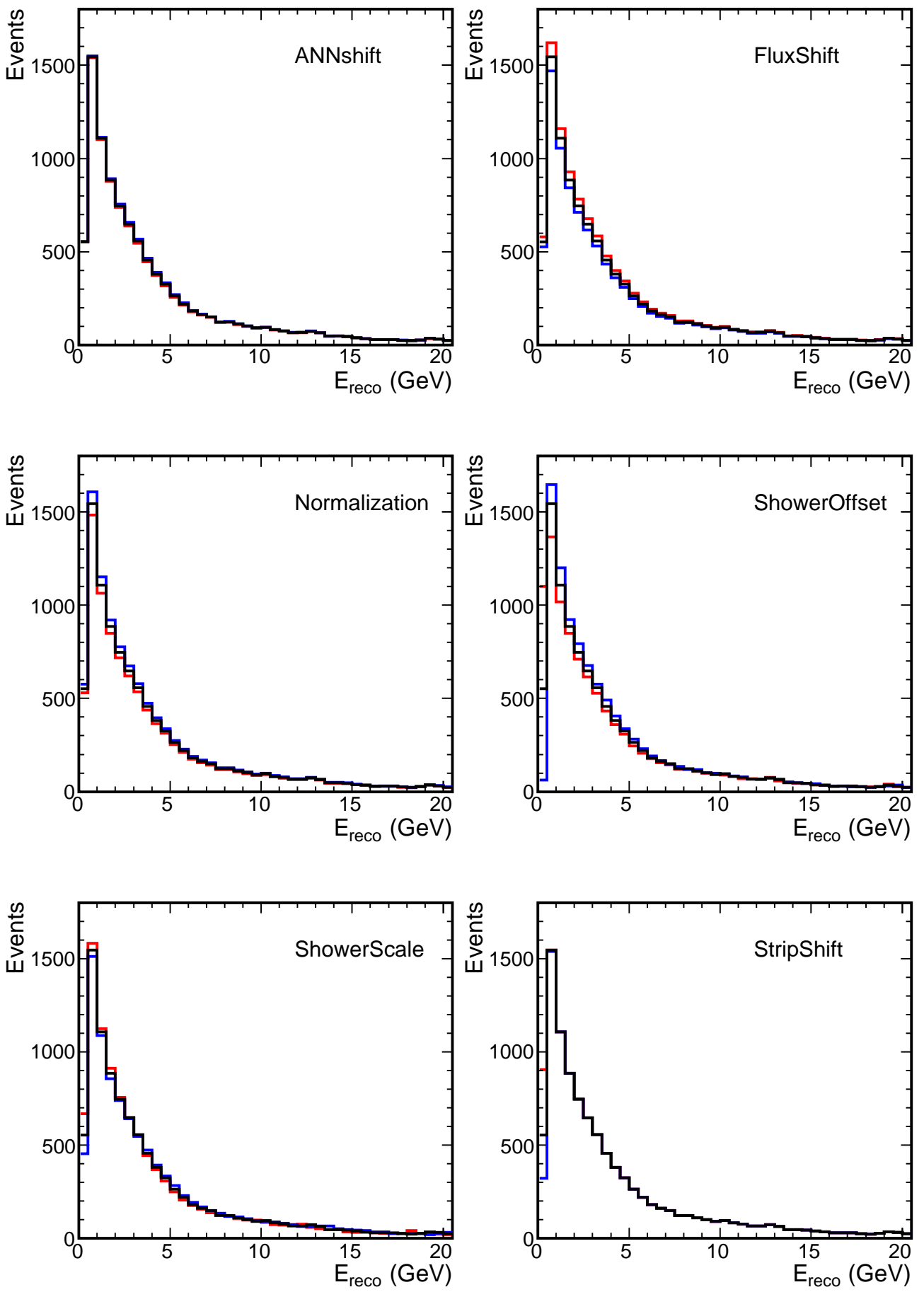

Figure 6.6: Systematically shifted neutral current spectra for the low energy beam. The red and blue histograms show the $\pm \sigma$ shifted neutral current spectrum for a given systematic effect. The black line shows the original Monte Carlo distribution. 

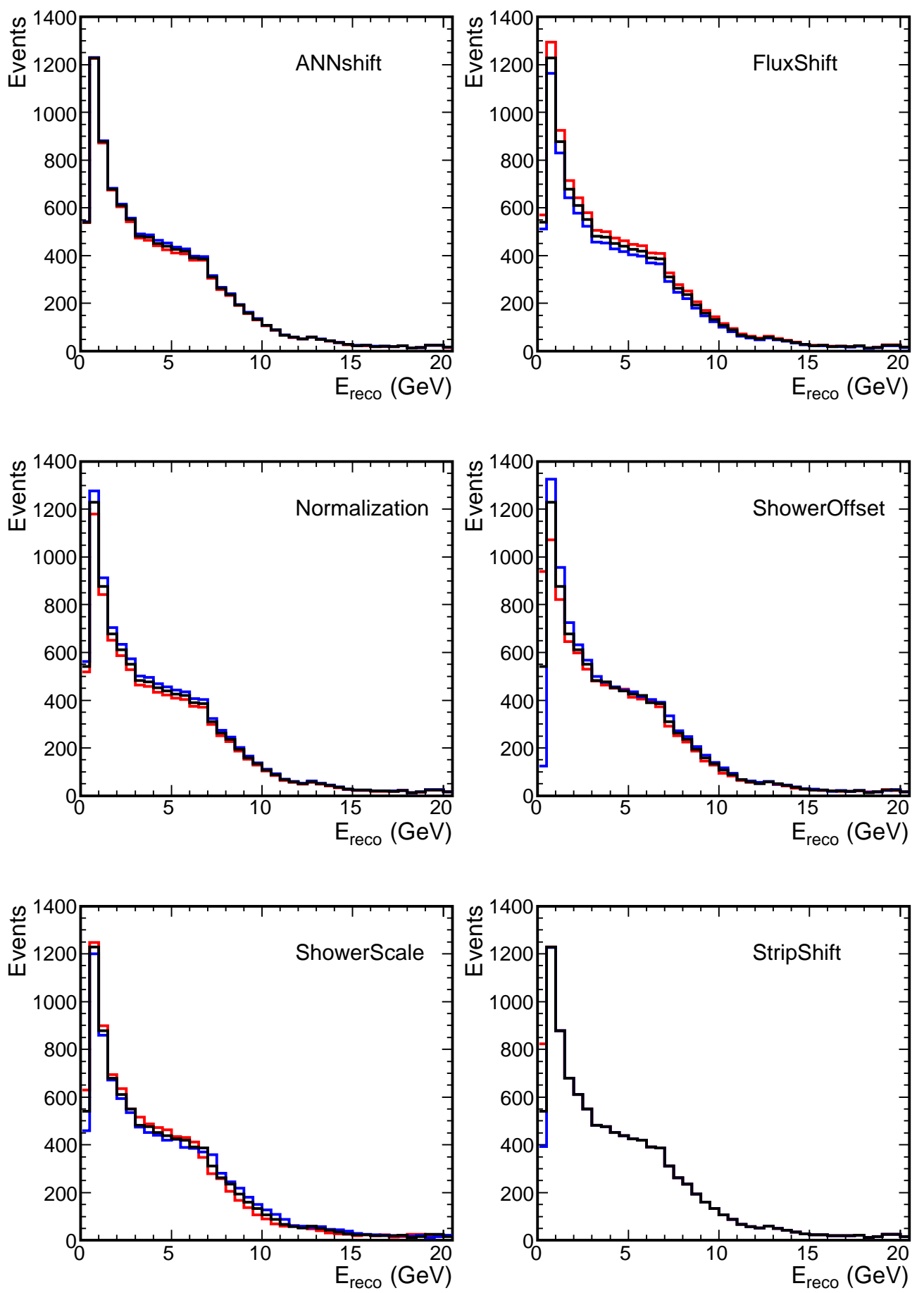

Figure 6.7: Systematically shifted neutral current spectra for the medium energy beam. The red and blue histograms show the $\pm \sigma$ shifted neutral current spectrum for a given systematic effect. The black line shows the original Monte Carlo distribution. 

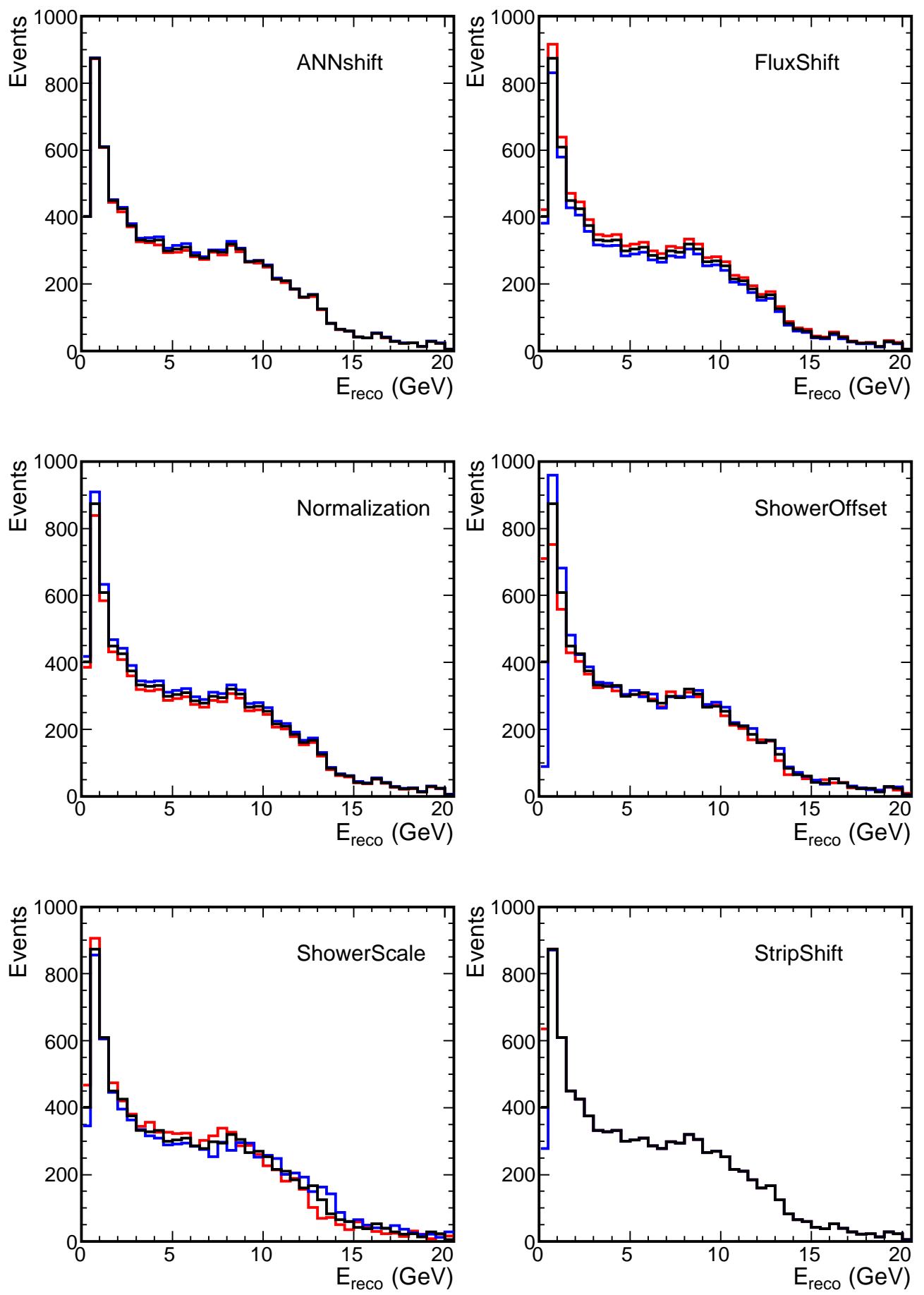

Figure 6.8: Systematically shifted neutral current spectra for the high energy beam. The red and blue histograms show the $\pm \sigma$ shifted neutral current spectrum for a given systematic effect. The black line shows the original Monte Carlo distribution. 


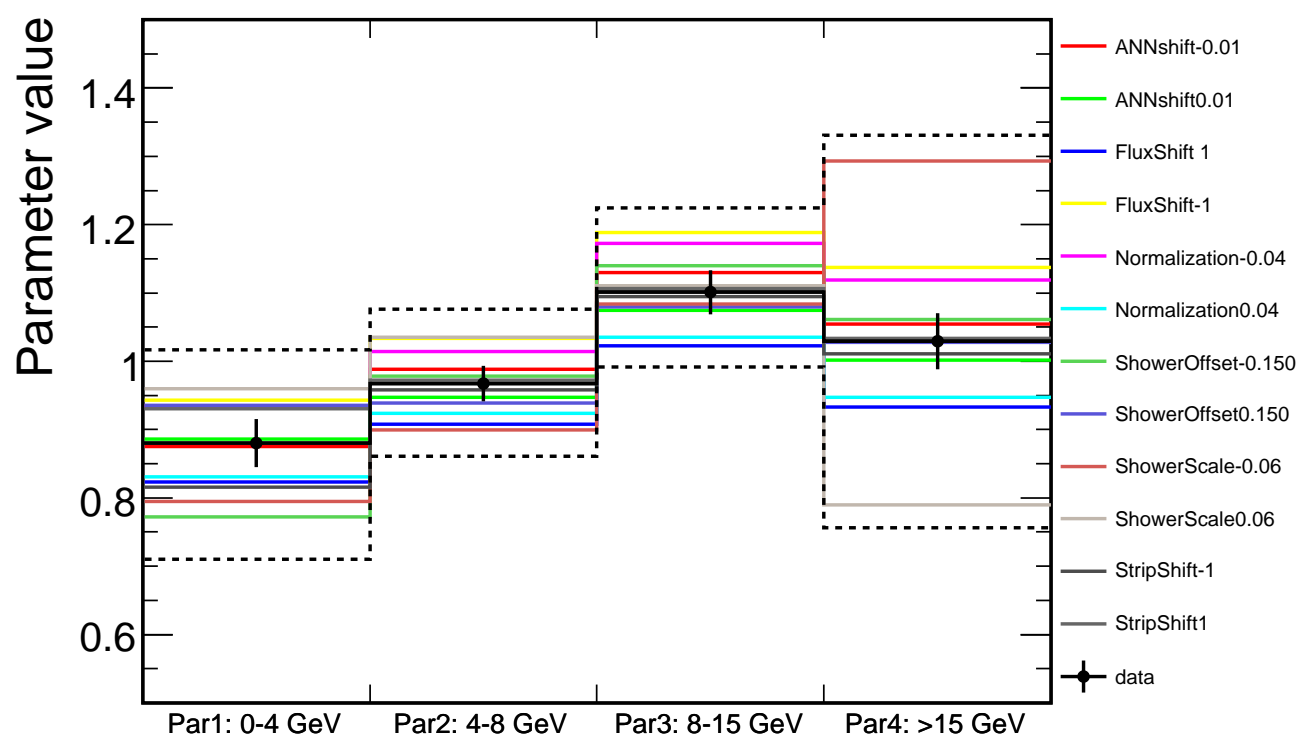

Fit parameters

Figure 6.9: Systematic errors on the cross-section scale parameters. The coloured lines show the effect of varying various systematic effects in the Monte Carlo when fitting the data. The dashed line shows the quadratic sum of all the systematic effects considered. The markers show the best-fit to the data with statistical errors.

at low energies, i.e. cross-section scale parameter 1, and become negligible at high energies. At high energies (parameter 4) the dominant error by far is the uncertainty on the shower energy scale.

The above systematic shifts are combined to obtain a single number for the systematic uncertainty on the cross-section scale measurement. The different systematic effects considered are treated as independent and thus summed in quadrature. Figure 6.9 shows the best-fit values for each systematically modified Monte Carlo set. The combined systematic error band is shown as a dashed line.

The final results for the measurement of neutral current cross-section scale factors in the MINOS Near Detector are:

$$
\begin{array}{ll}
\text { Par } 1(0-4 \mathrm{GeV}): & 0.880 \pm(0.035)_{\text {stat }}(+0.136-0.170)_{\text {syst }} \\
\text { Par } 2(4-8 \mathrm{GeV}): & 0.967 \pm(0.026)_{\text {stat }}(+0.107-0.105)_{\text {syst }}
\end{array}
$$




$$
\begin{array}{ll}
\text { Par } 3(8-15 \mathrm{GeV}): & 1.101 \pm(0.033)_{\text {stat }}(+0.120-0.106)_{\text {syst }} \\
\text { Par } 4(>15 \mathrm{GeV}): & 1.029 \pm(0.041)_{\text {stat }}(+0.301-0.272)_{\text {syst }}
\end{array}
$$

The first, symmetric error quoted on each of the parameters is the statistical uncertainty which includes the statistical error on both data and Monte Carlo simulation. The second set of uncertainties are the systematic errors evaluated in the previous section.

It is evident that this measurement is dominated by systematic uncertainties. Within these uncertainties, all neutral current cross-section scale parameters are consistent with 1 . This means, no departure from the neutral current cross-section values implemented in the NEUGEN Monte Carlo simulation is observed in the Near Detector data.

\subsection{Conclusion}

A fitting technique to extract neutral current cross-section parameters from the MINOS Near Detector data has been developed. The technique is shown to perform without bias and applied to the full Near Detector data sample. The extracted cross-section scale factors are consistent with the default values in the MINOS Monte Carlo simulation.

In the future, this method could be used to improve the Monte Carlo prediction of the Far Detector neutral current spectrum. It is important to note that the systematic uncertainties on the Far Detector prediction will be much smaller than the errors on the cross-section parameters. Many effects affecting the cross-section fit presented in this chapter will not affect the Far Detector prediction since they cancel between the two MINOS detectors. Two obvious examples for this are the absolute normalisation and the absolute shower energy scale. Instead, relative differences between the detectors (for example the uncertainty on the detector masses) become important. 
With this, we leave the description of the neutral current analysis. As alluded to, there will be work in the future on applying the neutral current cross-section fit results to a Far Detector prediction and ultimately a fit for sterile neutrinos. This is however beyond the scope of this thesis.

The next and final chapter is devoted to the charged current disappearance measurement which was mentioned several times before. Special emphasis will be given to the description and evaluation of systematic uncertainties for this measurement. 


\section{Chapter 7}

\section{Muon neutrino disappearance}

\section{analysis}

This chapter details the methods and results of the first MINOS $\nu_{\mu}$ disappearance measurement, which was published in Physical Review Letters in November 2006 [28]. I will briefly summarise the key features of the analysis techniques used (sections 7.1-7.5), before describing the study of systematic errors for this result in more detail. A collaboration decision was taken to conduct the evaluation of systematic errors for this analysis independent from the authors of the analysis software. This evaluation effort, described in section 7.6, was based in Oxford and is therefore an important part of this thesis.

The principle of the $\nu_{\mu}$ disappearance measurement is as follows: $\nu_{\mu}$ charged current neutrino interactions are measured in the two MINOS detectors. The Near Detector measurement is used to improve the flux and cross-section predictions in the Monte Carlo simulation. This improved Monte Carlo is used to predict the neutrino energy spectrum at the Far Detector for a "no-oscillations" scenario. Finally, the Far Detector measurement is fitted to extract the neutrino oscillation parameters.

As described in chapter 2, the survival probability is well approximated by a 
two flavour $\nu_{\mu} \rightarrow \nu_{\tau}$ oscillation scenario. In this case, it is given by:

$$
P\left(\nu_{\mu} \rightarrow \nu_{\mu}\right)=1-\sin ^{2}\left(2 \theta_{23}\right) \sin ^{2}\left(1.27 \frac{\Delta m_{32}^{2} L(\mathrm{~km})}{E(\mathrm{GeV})}\right)
$$

where the relevant mass splitting is referred to as $\Delta m_{32}^{2}$. Because of the smallness of the solar $\Delta m_{12}^{2}$, MINOS cannot, in fact, distinguish between $\Delta m_{32}^{2}$ and $\Delta m_{31}^{2}$.

\subsection{Charged current event selection}

An important part of this measurement is the selection of a very pure sample of $\nu_{\mu}$ charged current events. Neutral current interactions are not flavour specific and are therefore a background to this analysis. $\nu_{\tau}$ charged current events are a negligible contribution to the sample since, at the NuMI beam energies, phase space and cross-section heavily suppress the production of $\tau$ leptons.

Whenever Monte Carlo distributions are shown in this chapter, a tuned hadron production model which makes use of Near Detector measurements at different beam settings is used. This hadron production tuning is explained in more detail in section 7.2 .

The CC event selection proceeds in two stages. First, the event has to pass the following pre-selection cuts: The event must contain one or more well-reconstructed tracks, which pass basic track quality cuts. The track vertex has to be inside the fiducial volume of the detectors. In the case of the Near Detector, a cylinder of $1 \mathrm{~m}$ radius and $4 \mathrm{~m}$ length, located in the fully instrumented detector region and centred with respect to the beam is used. In the Far Detector, a cylinder of radius $3.7 \mathrm{~m}$ is used. In addition, the vertex must have a minimum distance of $50 \mathrm{~cm}$ from the front and end of the supermodules. Finally, the track vertex must be $2 \mathrm{~m}$ upstream of the end of supermodule 2, to allow for a sufficient track length for momentum determination. The reconstructed charge must be negative and 

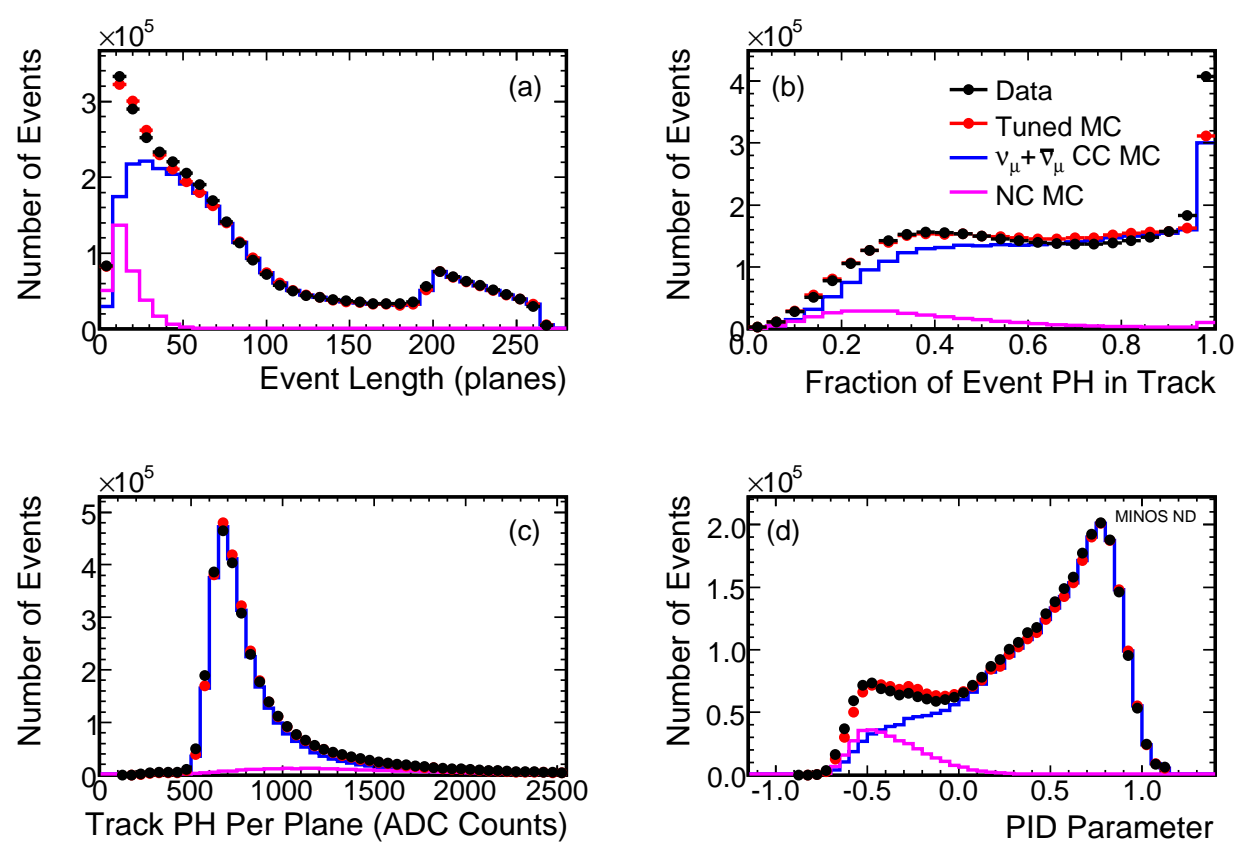

Figure 7.1: PDF variables and PID parameter. Data and tuned MC predictions are shown for event length (a), track pulse height fraction (b), and average pulse height per track plane (c), as well as the PID variable derived from these quantities (d), in the Near Detector. The Monte Carlo NC and CC breakdowns are shown in magenta and blue. The distributions look similar for the Far Detector.

the energy less than $30 \mathrm{GeV}$, in order to preferentially select neutrinos originating from $\pi^{+}$decay, rather than $\pi^{-}$or $K^{ \pm}$, since the $\pi^{+}$flux is the best understood parent flux in the NuMI beam.

The second step is a likelihood method for charged current / neutral current separation. It makes use of three probability density functions (PDFs), constructed from event reconstruction variables, which show differences between CC and NC interactions. They are:

- event length

The length of the event in detector planes. This variable is sensitive to $p_{\mu}$.

- track PH fraction

Fraction of total pulse height reconstructed as part of the track. For CC events, this is related to the momentum transfer $y$.

- track PH per Plane 
The average pulse height per plane on the track. This is related to the energy $\operatorname{loss} \frac{d E}{d x}$.

Probability density functions (PDFs) for Near Detector Monte Carlo and data are shown in Figure 7.1 (a)-(c). There is a clear distinction between the two different event categories in all three variables. The probability for being "CClike" or "NC-like" is defined as the product of the individual probabilities $f_{i}(x)$ :

$$
P_{C C, N C}=\prod_{i=1}^{3} f_{i}(x)_{C C, N C}
$$

These probabilities are combined into a particle identification (PID) variable for the final classification:

$$
P I D=-\left(\sqrt{-\log P_{C C}}-\sqrt{-\log P_{N C}}\right)
$$

Figure 7.1 (d) shows the PID distribution in Near Detector data and Monte Carlo. Events which are more likely to be $\mathrm{CC}$ are assigned positive values and vice versa for events more likely to be NC. To achieve maximum efficiency for low energy charged current events, a cut at a PID value of -0.1 (ND) and -0.2 (FD) was chosen for this analysis. Events with a smaller PID value than the cut given above are considered NC-like and therefore not considered in the oscillation analysis. The two different cut values were chosen in order to obtain similar CC selection efficiencies and sample purities in both detectors. Figure 7.2 shows the PID selection efficiencies and purities as a function of reconstructed energy for both detectors.

In the Far Detector, two additional cuts are used to further reduce background from cosmic rays. Firstly, a cut is imposed on the direction of the reconstructed track. This is to ensure that the track is consistent with the beam direction rather than a cosmic ray which entered the detector through a gap. We require the cosine with respect to the beam direction to be greater than 0.6. This corresponds to an 

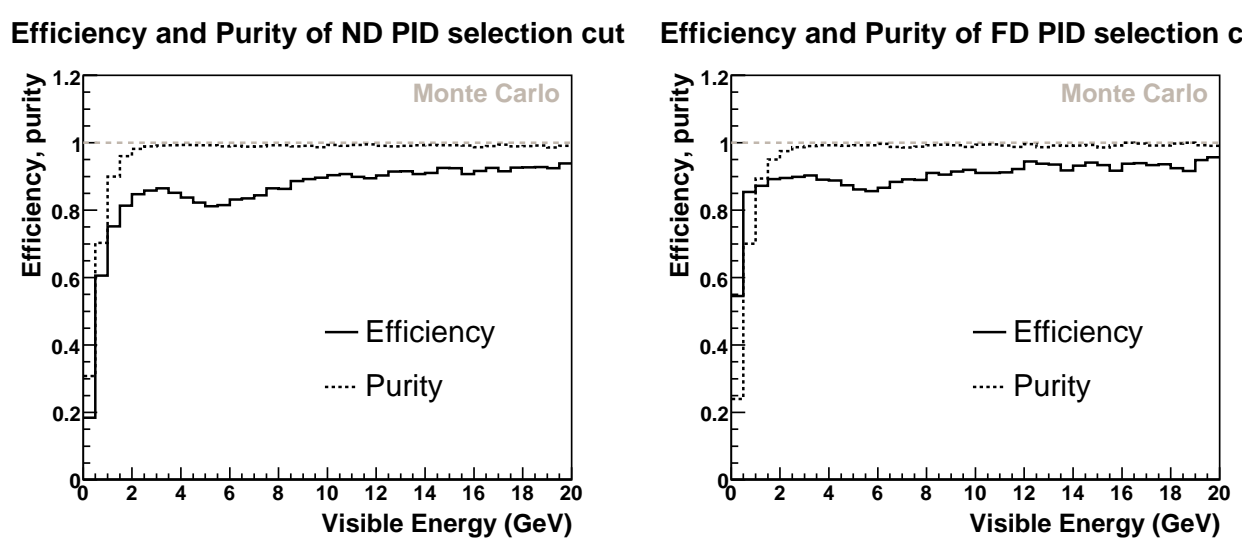

Figure 7.2: PID selection efficiencies and purities. in the Near Detector (left) and the Far Detector (right). The efficiency is shown as a solid, the purity as a dashed line.

angle of $53^{\circ}$. Secondly, the event time must be within a $50 \mu s$ window around the predicted time of the beam spill.

The overall efficiencies for selecting charged current neutrino interactions, using the set of cuts described, is $67.3 \%$ for the Near Detector and $74.3 \%$ for the Far Detector. The final Far Detector sample includes 384 neutrino candidates for a total exposure of $1.27 \times 10^{20}$ protons on target.

\subsection{Neutrino flux tuning}

One important uncertainty for the prediction of the Far Detector neutrino energy spectrum comes from the incomplete knowledge of the hadron production in the NuMI target, resulting in an uncertainty on the neutrino flux. In this section, I briefly present a method using Near Detector measurements in order to tune the Monte Carlo prediction. A more detailed description can be found in [105].

Experimental data on hadron production from a thick carbon target using a $120 \mathrm{GeV}$ proton beam is very sparse. It is therefore not surprising that hadron production is a major source of disagreement between data and Monte Carlo in MINOS. The main source of uncertainty on the neutrino flux at both detectors is 
the hadron yield $\mathrm{d}^{2} N / \mathrm{d} x_{F} \mathrm{~d} p_{T}$ as a function of transverse momentum $p_{T}$ and $x_{F}$. $x_{F}$ is the Feynman scaling variable, defined as follows:

$$
x_{F}=\frac{p_{L}}{p_{L, \max }}=\frac{2 p_{L}}{\sqrt{s}}
$$

$p_{L}$ refers to the longitudinal momentum of the hadron and $\sqrt{s}$ to the centre-ofmass energy of the interaction. $p_{T}$ and $x_{F}$ are important because of the limited $p_{T}$ acceptance of the magnetic focusing horns and the relation between $x_{F}$ and the neutrino energy.

The neutrino flux tuning uses charged current neutrino energy spectra obtained with the selection algorithm presented in the previous section 7.1. By moving the NuMI target with respect to the horns and by varying the horn current, neutrino energy spectra in six different configurations were recorded. The target positions are referred to as low energy (LE10), medium energy (LE100) and high energy (LE250). Changing the target position and/or horn current results in a different energy spectrum by selecting different regions in the $\left(x_{F}, p_{T}\right)$ phase space of the parent pions.

The tuning method represents the underlying production yield, $\mathrm{d}^{2} N / \mathrm{d} x_{F} \mathrm{~d} p_{T}$, by an empirical function in $x_{F}$ and $p_{T}$. Additional parameters are added to account for beam focusing and detector modelling uncertainties. This function is then used to reweight the Monte Carlo in a $\chi^{2}$ fit to the Near Detector data, obtained with the different beam configurations.

The energy spectra and data/MC ratios for six different beam configurations before and after the tuning are shown in Figure 7.3. They are labelled by target position and horn current. It can be seen that the tuning improves the agreement between data and Monte Carlo significantly in all cases. 


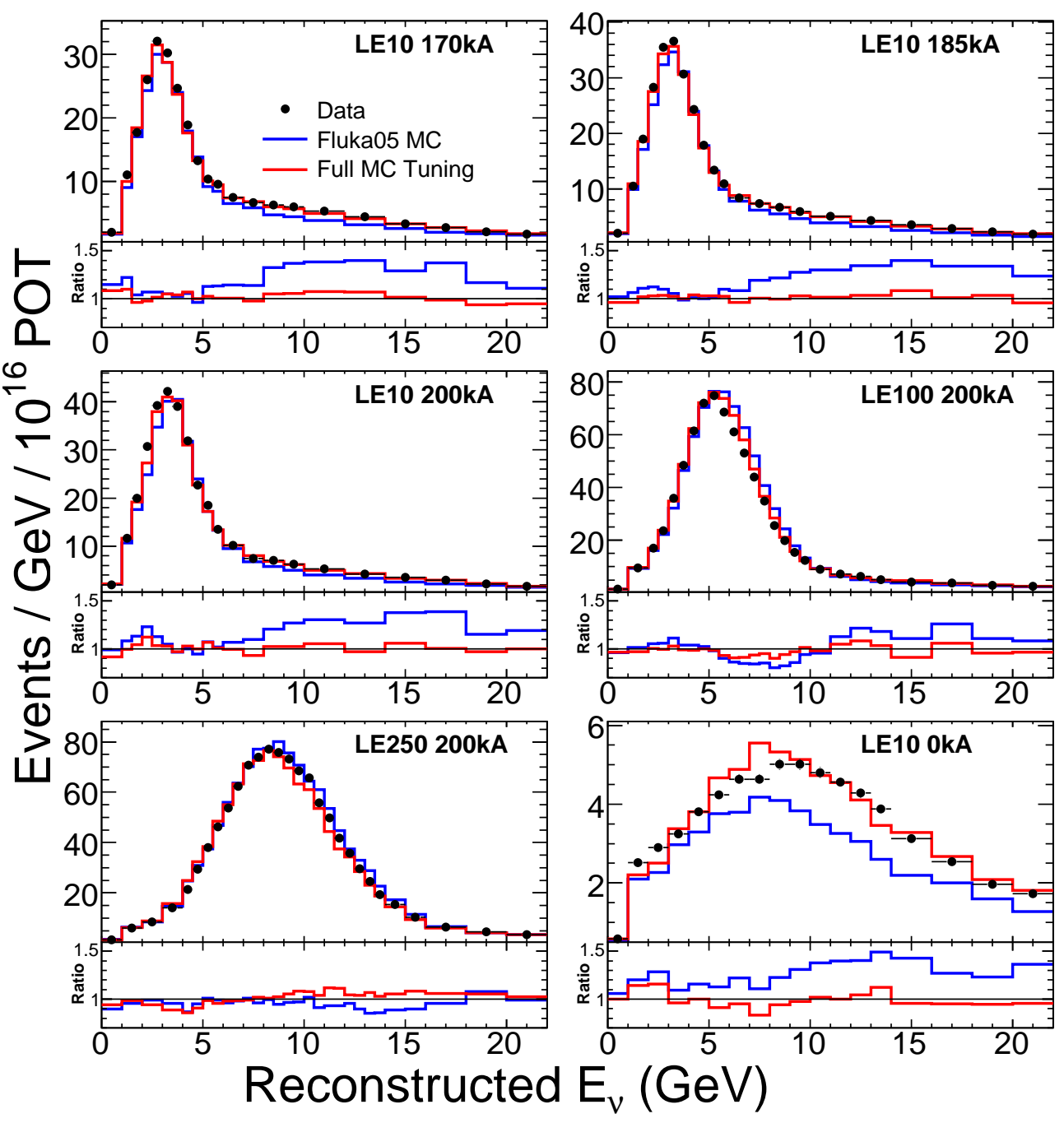

Figure 7.3: CC energy spectra for different beam configurations. Data and Monte Carlo energy spectra and their ratio are shown for six different beam configurations. The blue line corresponds to the untuned, the red line to the tuned hadron production model. The tuning improves the data/MC agreement in all cases. 


\subsection{Beam extrapolation to the Far Detector}

The reason for the two-detector design of MINOS is the fact that many systematic uncertainties cancel when doing a differential measurement. For this to work, it is necessary to correctly extrapolate the Near Detector measurement to the Far Detector. This is non-trivial since the two detectors cover different solid angles from the neutrino production target. The energy of the neutrino produced via the decay $\pi^{+} \rightarrow \mu^{+}+\nu_{\mu}$ in the NuMI beamline is related to its decay angle $\theta$ via two-body decay kinematics:

$$
E_{\nu}=\frac{0.43 E_{\pi}}{1+\gamma^{2} \theta^{2}}
$$

where $\gamma$ is the Lorentz boost factor and $\theta$ is the angle between the neutrino and the pion in the lab frame. For decays at the centre of the decay pipe, only neutrinos which emerge from the decay exactly forward can reach the Far Detector, whereas a range of angles are possible for the Near Detector. This means, the energy spectra in the two detectors are different. Furthermore, a data/Monte Carlo discrepancy observed at a certain energy in the Near Detector will contribute to different energies in the Far Detector. That means, for example, a $5 \%$ difference observed in an energy bin from 5-6 GeV cannot simply be corrected for by multiplying the same bin in the Far Detector spectrum.

Four different methods to achieve this extrapolation have been proposed in MINOS. The method adopted as the main method for our first publication in PRL is called "Beam Matrix Method". It was first proposed in [106]. The Beam Matrix method directly extrapolates the measured differences between Near Detector data and Monte Carlo to the Far Detector, using the appropriate beam kinematics. This is in contrast to other methods which fit model parameters, such as a shower energy scale or a cross-section correction, to the Near Detector data and apply the obtained fit values to the Far Detector MC. I will explain the Beam Matrix extrapolation technique in the remainder of this section. 
The Beam Matrix method extrapolates the Near Detector measurement in essentially three separate steps. Starting from a selected CC-like reconstructed energy spectrum, they are:

1. conversion from reconstructed to true neutrino energy, subtracting background, and correcting for efficiency

2. beam matrix conversion from Near Detector true energy to Far Detector true energy using kinematics and beamline geometry

3. conversion from true neutrino energy to reconstructed energy, correction for efficiency, and adding of background

In step 1, the neutral current background is subtracted from the CC-like sample using the selection purity determined from Monte Carlo. The resulting spectrum represents the reconstructed energy spectrum of true CC events. Subsequently, a "reco-to-true" matrix, obtained from Monte Carlo, is used to convert the reconstructed energy spectrum to a true neutrino energy spectrum. This true neutrino energy spectrum is corrected for reconstruction and selection efficiencies, in order to obtain the true neutrino energy spectrum of all CC interactions in the Near Detector. These steps are necessary since decay kinematics and beam line geometry affect the true energy of the neutrino.

Step 2 uses an $N \times N$ matrix, called the Beam Matrix, to extrapolate the Near Detector CC neutrino energy spectrum to its Far Detector equivalent. $N$ corresponds to the number of energy bins used. This is the core of the method. The Beam Matrix incorporates the best of our knowledge on pion and kaon decay kinematics, the geometry of the beam line, and its focusing elements. It is normalised so as to act as a probability matrix. This way, the matrix element $B_{i j}$ represents the probability that, given a neutrino interaction in energy bin $i$ at the Near Detector, $B_{i j}$ neutrino interactions are expected in energy bin $j$ at the Far 

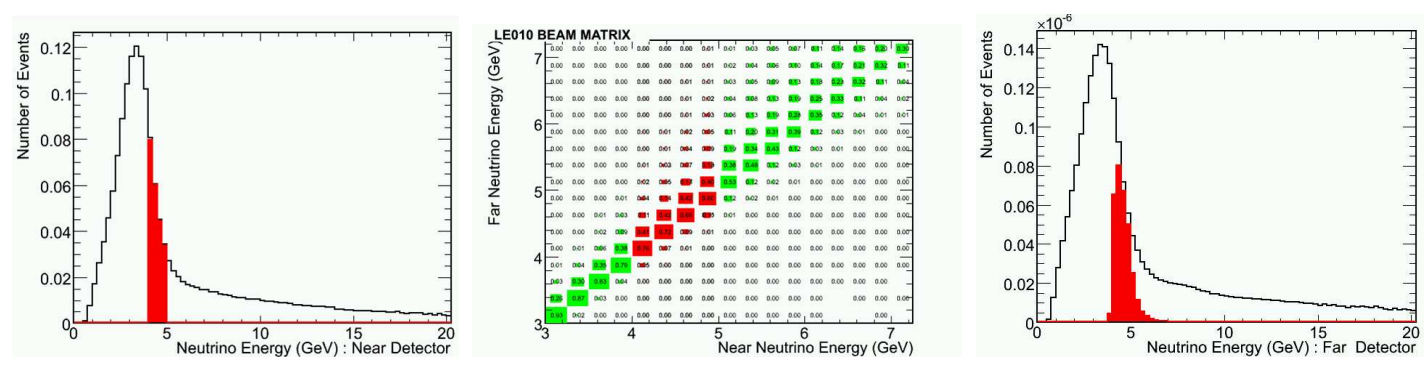

Figure 7.4: The operation of the Beam Matrix. This sketch shows how a given energy bin in the Near Detector spectrum is extrapolated to the Far Detector using a 2-dimensional matrix (left to right). The matrix is close to diagonal but some migration to neighbouring energy bins occurs due to the different angular acceptances of the two detectors.

Detector. The Beam Matrix is constructed from the GNuMI beam Monte Carlo simulation and the neutrino differential cross-sections. The operation of the Beam Matrix is shown pictorially in Figure 7.4.

Step 3 is the inverse of step 1, converting the extrapolated true neutrino energy spectrum at the Far Detector back to reconstructed energy. Once again, efficiency and purity corrections are applied in order to account for reconstruction and selection inefficiencies as well as backgrounds. The resulting Far Detector reconstructed energy spectrum can be compared to the data and fitted for oscillations or other more exotic models.

The main advantage of the Beam Matrix method is that it fully exploits the cancellation of systematic uncertainties between the two detectors while properly taking into account the decay kinematics. This is particularly true for beam modelling related uncertainties. However, due to the near diagonality of the matrix, it is also approximately true for cross-section uncertainties. Figure 7.5 illustrates the success of the method in accurately predicting the Far Detector spectrum. 

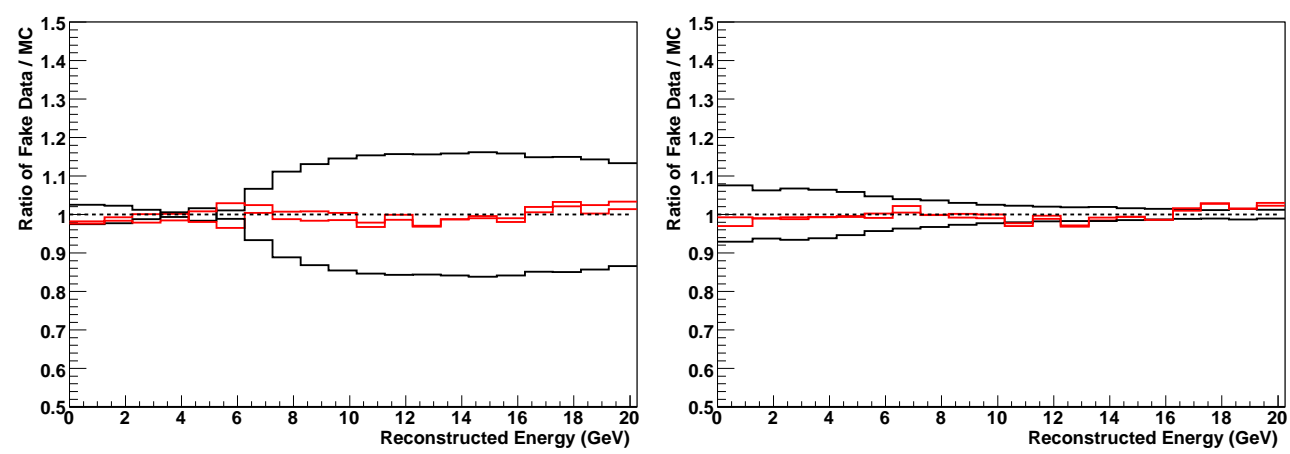

Figure 7.5: Beam Matrix predictions for systematically shifted Monte Carlo. The left figure shows a modified beam model, the right figure modified cross-sections. The black lines show the ratio of systematically shifted Monte Carlo to standard Monte Carlo in the Near Detector for shifts in both directions. The red lines show the ratio of predicted to true spectrum in the Far Detector. Despite large variations, the method predicts the Far Detector spectrum accurately.

\subsection{Far Detector Oscillation Fit}

After extrapolation, the predicted Far Detector spectrum is fitted to the measured data in order to extract oscillation parameters. Oscillations are incorporated into the Monte Carlo in step 3 of the extrapolation method described in the previous section. Oscillations according to the two-flavour approximation are applied to the Far Detector true energy spectrum before the conversion back to reconstructed energy. The oscillation parameters $\Delta m_{32}^{2}$ and $\sin ^{2}\left(2 \theta_{23}\right)$ are varied in a 2-dimensional grid search. The best-fit point is determined using a $\chi^{2}$ statistic appropriate for small numbers of events [41]:

$$
\chi^{2}\left(\Delta m_{32}^{2}, \sin ^{2}\left(2 \theta_{23}\right), \alpha_{j}\right)=\sum_{i+1}^{n b i n s} 2\left(e_{i}-o_{i}\right)+2 \ln \left(o_{i} / e_{i}\right)+\sum_{j=1}^{n s y s t} \frac{\Delta \alpha_{j}^{2}}{\sigma_{\alpha_{j}}^{2}}
$$

where $e_{i}$ and $o_{i}$ are the number of expected and observed events in energy bin $i$ respectively. The fit is performed using 10 bins in the range $0-10 \mathrm{GeV}, 4$ bins from 10 to $18 \mathrm{GeV}$, and one bin for the range $18-30 \mathrm{GeV}$. The $\alpha_{j}$ represent systematic parameters which are included in the fit. $\Delta \alpha_{j}$ is the difference from the nominal value for systematic parameter $\alpha_{j}$ and $\sigma_{\alpha_{j}}$ is the estimated 1-sigma uncertainty 
on parameter $\alpha_{j}$. The functional form of the systematic error terms allows the fit to vary the values for the systematic parameters at the expense of increasing the $\chi^{2}$ value. These terms, referred to as penalty terms, represent a common way of including systematic uncertainties in the fit, used for example in [107]. A detailed description of the systematic uncertainties considered in this analysis is given in section 7.6 later in this chapter.

\subsection{Oscillation result}

Figure 7.6 (a) shows the Far Detector data and the null hypothesis Monte Carlo prediction from the Beam Matrix method, as well as the best-fit prediction, shown as a dashed line. Figure 7.6 (b) shows the data/MC ratio with its characteristic oscillation "dip". The best-fit values for the oscillations parameters are $\Delta m_{32}^{2}=$ $2.74 \times 10^{-3} \mathrm{eV}^{2}$ and $\sin ^{2}\left(2 \theta_{23}\right)=1.0$. The errors on the parameters are determined using a Gaussian approximation. The allowed parameter ranges are $2.48 \times 10^{-3}<$ $\Delta m_{32}^{2}<3.18 \times 10^{-3}$ and $\sin ^{2}\left(2 \theta_{23}\right)>0.87$ at $68 \%$ confidence level. This includes systematic uncertainties as described in 7.6.2. The value of $\chi^{2}$ at the best-fit point is 20.5 for 13 degrees of freedom. This corresponds to a $\chi^{2}$ probability $P\left(\chi^{2}\right.$, n.d.f. $)=8.9 \%$.

Figure 7.7 shows the allowed regions in parameter space at 68, 90, and $99 \%$ confidence level. The contours were computed using the $\Delta \chi^{2}$ Gaussian approximation $\left(\Delta \chi^{2}=2.3,4.6,9.2\right)$.

\subsection{Systematic error evaluation}

The systematic errors for this analysis were evaluated separately for the four different methods proposed to the collaboration. The decision to adopt the Beam Matrix method as the primary extrapolation method for the first MINOS mea- 

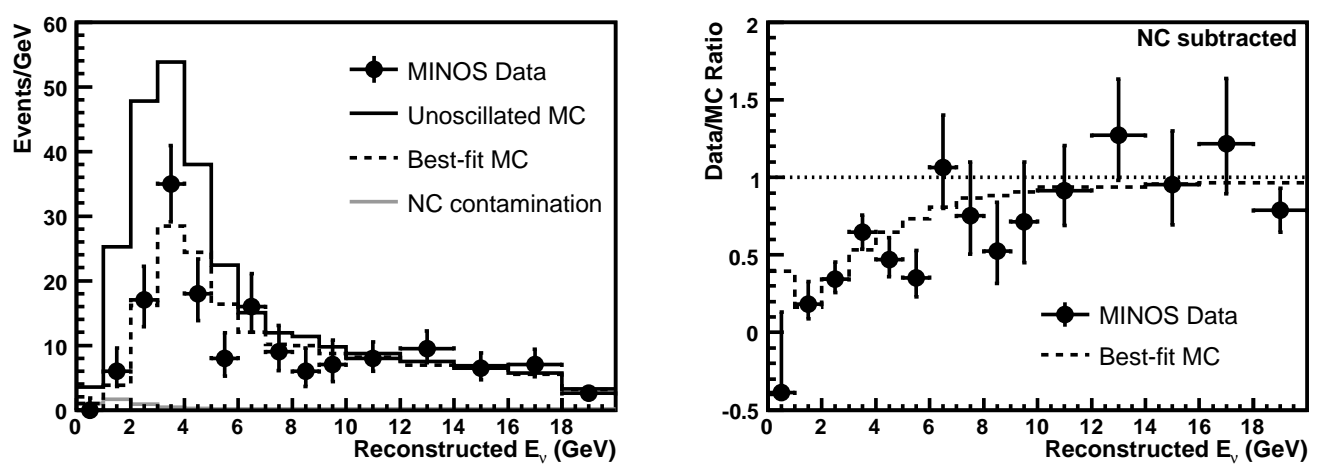

Figure 7.6: Far Detector data and Monte Carlo energy spectra including the bestfit spectrum shown as a dashed line (left). The right figure shows the data/MC ratio using the unoscillated Monte Carlo prediction, which exhibits the characteristic oscillation "dip" The dashed line is obtained using the best-fit parameters.

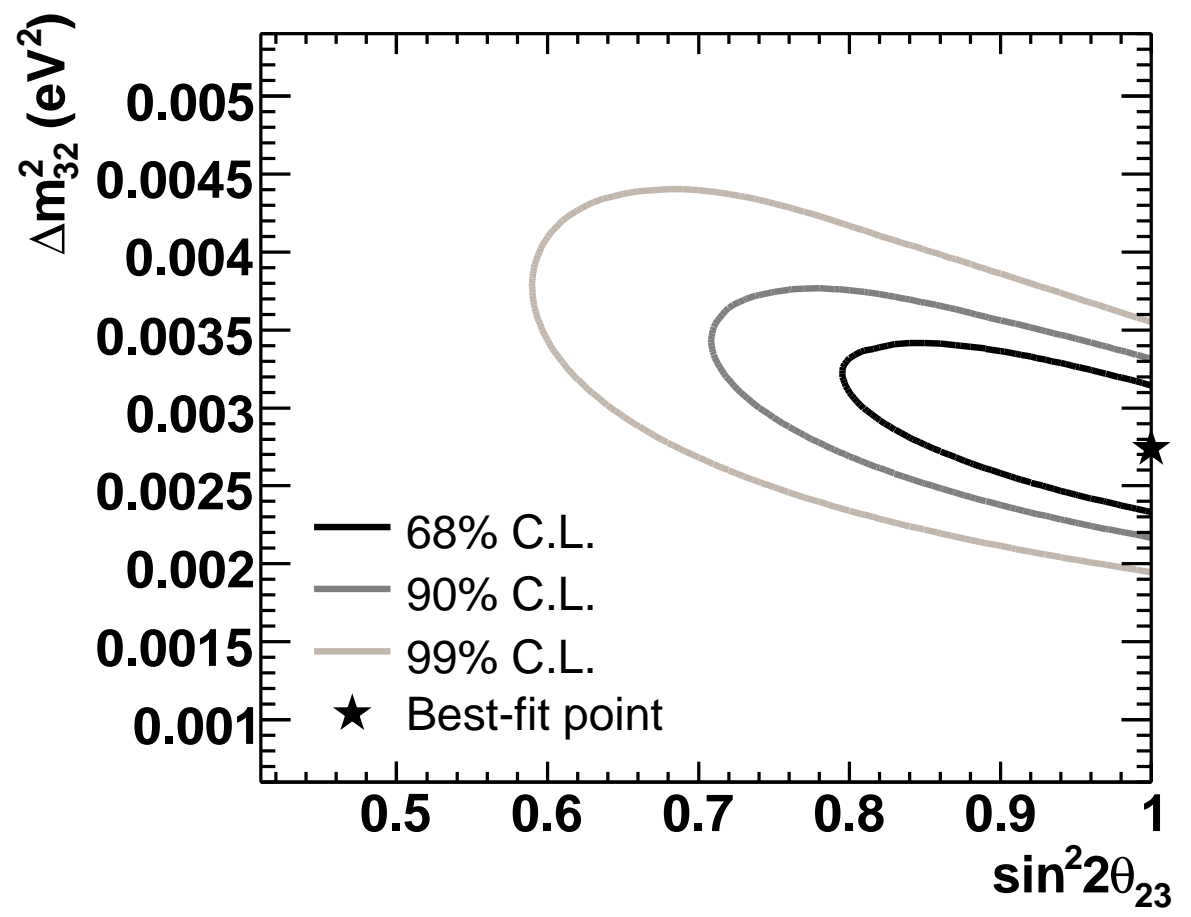

Figure 7.7: Allowed regions in the $\left(\Delta m_{32}^{2}, \sin ^{2}\left(2 \theta_{23}\right)\right)$ plane at $60,90,99 \%$ C.L. The best-fit point is marked by a star at $\Delta m_{32}^{2}=2.74 \times 10^{-3} \mathrm{eV}^{2}$ and $\sin ^{2} 2 \theta_{23}=$ 1.0. Systematic uncertainties, described in section 7.6.2, are included in the $\chi^{2}$ expression. 
surement was taken in light of the results of this evaluation exercise.

For the sake of brevity, we will focus on the systematic error evaluation for the Beam Matrix method. The systematic uncertainties considered in this analysis fall into three main categories: beam simulation, neutrino cross-sections and interaction modelling, and detector uncertainties. The second category includes uncertainties related to the detailed simulation of neutrino interactions in matter. The third category includes calibration uncertainties as well as reconstruction and selection related systematics.

The evaluation of the effects of these uncertainties on the oscillation parameter measurement was conducted in two separate steps. In the first step, the impact of different systematic uncertainties was compared to establish which were the dominant contributions to the total error. This information was also used in the decision for the primary extrapolation method for MINOS. In the second step, the dominant uncertainties were included in the fit by means of penalty terms to the $\chi^{2}$ definition (equation (7.6) in section 7.4).

\subsubsection{Fake data study for individual systematic errors}

To evaluate the importance of different systematic uncertainties, a fake data study was conducted. Fake data samples were created by shifting a Monte Carlo sample by $\pm 1 \sigma$ in a given systematic parameter. The so created fake data samples take the place of real data in the analysis. Both Near Detector and Far Detector fake data samples were created. The Far Detector fake data samples were oscillated using the best-fit parameters from a statistics only fit (i.e. without the systematic error terms in the $\chi^{2}$ definition): $\Delta m_{32}^{2}=2.72 \times 10^{-3} \mathrm{eV}^{2}$ and $\sin ^{2}\left(2 \theta_{23}\right)=1.0$. The complete analysis chain was applied to the fake data samples and the shifts in the best-fit parameters were recorded for each sample. The systematic parameters considered in the study and their magnitudes are listed in Table 7.1. 


\begin{tabular}{l|c|l}
\hline Systematic parameter & Shift & Notes \\
\hline \hline Beam tuning residual & $\pm 50 \%$ & $\begin{array}{l}50 \% \text { of the correction assumed } \\
\text { as uncertainty }\end{array}$ \\
\hline CCMA & $\pm 10 \%$ & axial mass in form factor \\
kno_1i2 & $\pm 10 \%$ & KNO scaling parameters \\
kno_1i3 & $\pm 20 \%$ & \\
Shower energy & $\pm 10 \%$ & intra-nuclear rescattering \\
Shower energy offset & $\pm 150 \mathrm{MeV}$ & \\
\hline Absolute shower calibration & $\pm 5.6 \%$ & \\
Relative shower calibration & $\pm 2 \%$ & calibration between detectors \\
Track energy & $\pm 2 \%$ & muon momentum scale \\
Normalisation & $\pm 4 \%$ & N/F reconstruction efficiencies \\
PID cut & \pm 0.02 & \\
NC background & $\pm 50 \%$ & \\
\hline
\end{tabular}

Table 7.1: Systematic parameters considered in the fake data study, loosely grouped into the categories beam simulation, neutrino interaction simulation, and detector related. The middle column shows the size of the shifts applied to the nominal Monte Carlo.

The Beam Tuning systematic refers to the $x_{F}, p_{T}$ reweighting procedure described in section 7.2. It results in changes to the reconstructed energy spectrum of $\sim 10 \%$ in the peak region and $\sim 30 \%$ in the tail. A somewhat conservative error of $50 \%$ of the correction has been used in this study.

The parameters $C C M A$ and kno_xyz are neutrino cross-section parameters in the NEUGEN Monte Carlo simulation. CCMA refers to the axial vector mass parameter in the form factor for quasi-elastic and resonance processes and kno_xyz are scaling parameters for different exclusive processes. The errors of $10 \%$ on axial mass and $20 \%$ on the cross-section for low-multiplicity DIS events reflect the current uncertainties on neutrino scattering cross-sections.

The parameters Shower energy and Shower energy offset reflect the uncertainty on the simulation of an effect which is known as intra-nuclear rescattering. This is the same effect as described in the context of the neutral current cross-section fit in chapter 6 . In this analysis, the observed effect was qualitatively modelled in 
terms of an offset and a scale factor to the reconstructed shower energy.

Absolute and relative shower energy as well as track energy reflect the estimated size of detector calibration uncertainties.

The uncertainty on the relative normalisation between the detectors is dominated by uncertainties on the near and Far Detector reconstruction efficiencies. The magnitude has been estimated using a visual scan of data and Monte Carlo in both detectors.

The PID cut parameter models the charged current selection uncertainty by moving the location of the PID cut by \pm 0.02 in the fake data samples.

The uncertainty on the $N C$ background is estimated from the comparison of shape and normalisation between the Near Detector PID distributions in data and Monte Carlo. The uncertainty was estimated to be $\pm 50 \%$.

Figures 7.8 and 7.9 show the effect of the various systematic shift on the reconstructed energy spectrum in the Near Detector for each of the systematic parameters considered.

Since these plots show the effects on the Near Detector spectrum, no plot is shown for the relative shower calibration error. This error was estimated by using a shifted Near Detector spectrum and a nominal Far Detector one, and vice versa. The systematic uncertainty due to the absolute shower calibration was estimated by using shifted spectra in both detectors.

The spectra in Figures 7.8 and 7.9 show how the different systematic parameters affect the reconstructed energy spectrum. On its own, this does not allow us to determine which systematic shifts cause the largest uncertainties on the oscillation parameters $\Delta m_{32}^{2}$ and $\sin ^{2}\left(2 \theta_{23}\right)$. To evaluate the effect of each systematic effect, the whole analysis chain including extrapolation and fitting was applied to the fake data sets.

Table 7.2 shows a compilation of the measured shifts in the best-fit values in $\Delta m_{32}^{2}$ and $\sin ^{2}\left(2 \theta_{23}\right)$ for the systematic parameters considered. The shifts in 

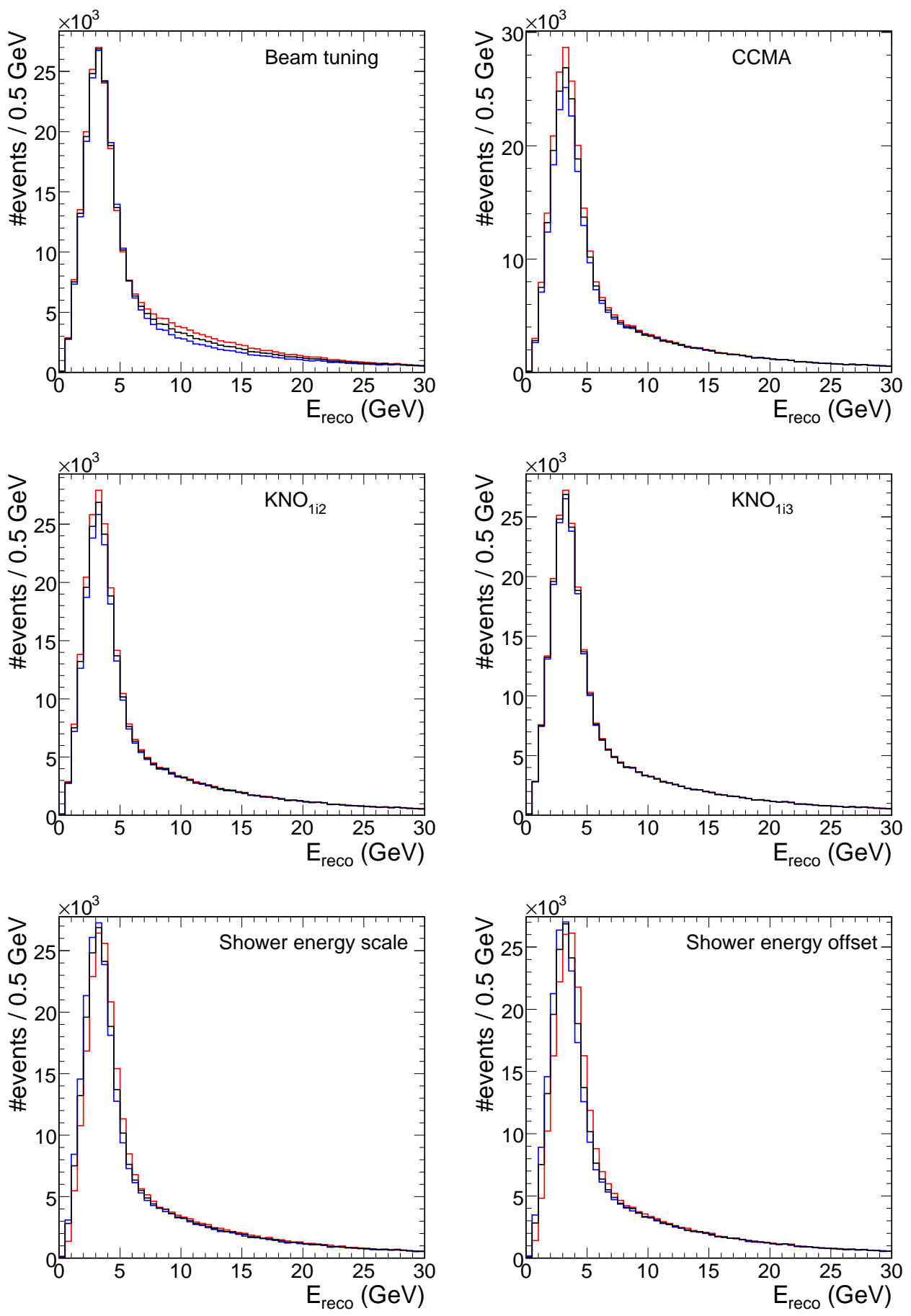

Figure 7.8: Systematically shifted fake data sets. Each figure shows the effect of one systematically shifted parameter on the Near Detector energy spectrum. The red and blue lines correspond to the $+1 \sigma$ and $-1 \sigma$ shifts respectively. 

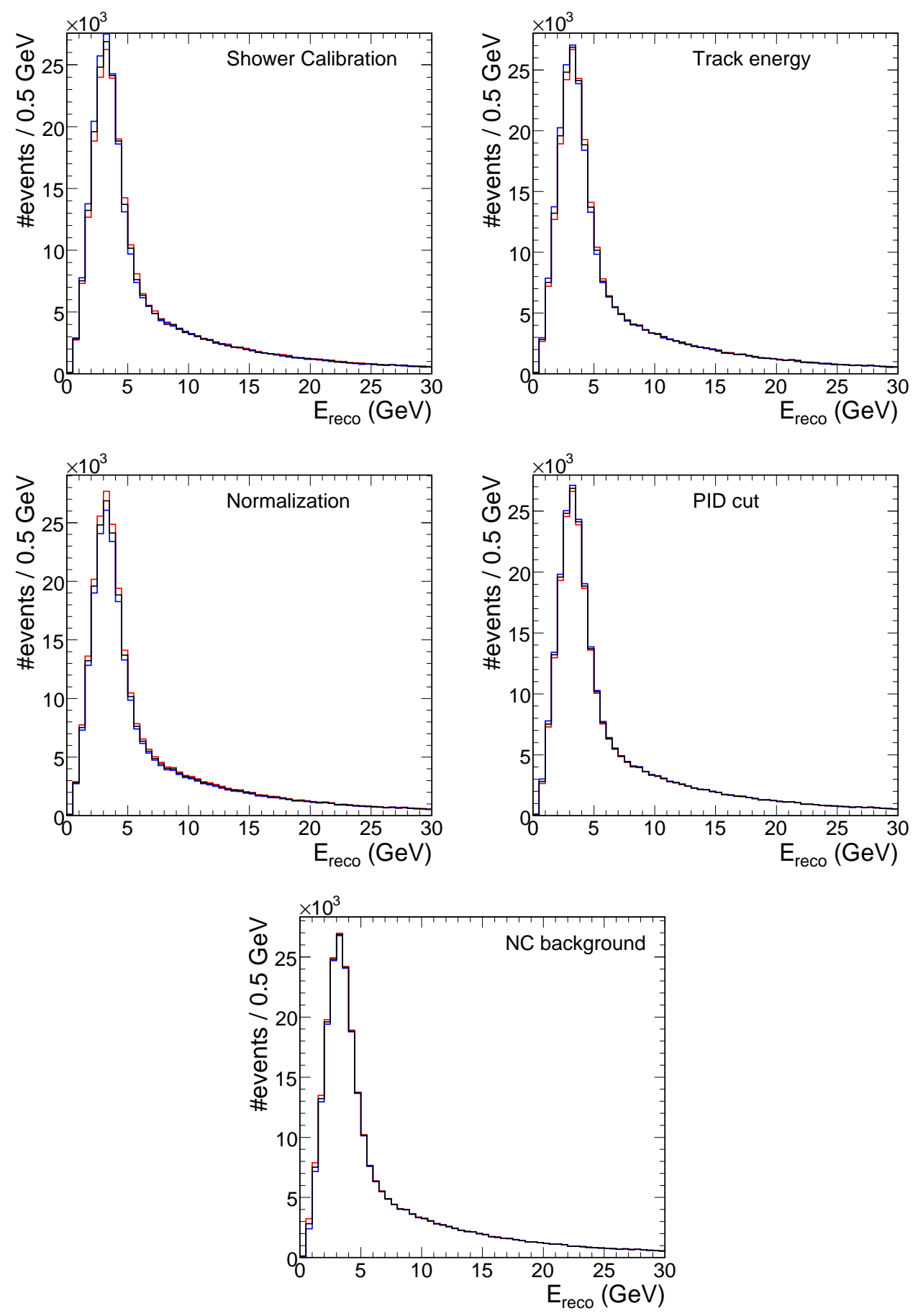

Figure 7.9: Systematically shifted fake data sets (cont'd). Each figure shows the effect of one systematically shifted parameter on the Near Detector energy spectrum. The red and blue lines correspond to the $+1 \sigma$ and $-1 \sigma$ shifts respectively. 


\begin{tabular}{l|c|c}
\hline Systematic parameter & $\left|\boldsymbol{\Delta}\left(\boldsymbol{\Delta} \boldsymbol{m}_{\mathbf{3 2}}^{\mathbf{2}}\right)\right| / \mathbf{1 0}^{-\mathbf{3}} \mathbf{e V}^{\mathbf{2}}$ & $\left|\boldsymbol{\Delta}\left(\mathbf{s i n}^{\mathbf{2}}\left(\mathbf{2 \theta}_{\mathbf{2 3}}\right)\right)\right|$ \\
\hline \hline Beam tuning residual & 0.0150 & 0 \\
\hline CCMA & 0.0125 & 0.0025 \\
kno_1i2 & 0.0100 & 0.0050 \\
kno_1i3 & 0.0025 & 0 \\
Shower energy & 0.0075 & 0.0500 \\
Shower energy offset & 0.1150 & 0.0325 \\
\hline Absolute shower calibration & 0.0325 & 0.0050 \\
Relative shower calibration & 0.0100 & 0.0050 \\
Track energy & 0.0325 & 0.0025 \\
Normalisation & 0.0500 & 0.0050 \\
PID cut & 0.0150 & 0.0075 \\
NC background & 0.0900 & 0.0500 \\
\hline
\end{tabular}

Table 7.2: The shifts in best-fit parameters for fake data sets. The results for the $+1 \sigma$ and $-1 \sigma$ shifts are averaged to obtain one number for each systematic parameter. Zero entries mean no shift has been observed. The largest contributions to the systematic uncertainty are: NC background, normalisation and the shower energy scale related parameters.

$\Delta m_{32}^{2}$ are given in multiples of $10^{-3} \mathrm{eV}^{2}$. The shifts for the $+1 \sigma$ and $-1 \sigma$ systematic variations have been averaged to obtain a single number for each systematic parameter. The granularity of the fit and, consequently, the minimum detectable shifts were $5 \times 10^{-6} \mathrm{eV}^{2}$ in $\Delta m_{32}^{2}$ and $5 \times 10^{-3}$ in $\sin ^{2}\left(2 \theta_{23}\right)$.

For many of the systematic parameters which were investigated, the Beam Matrix extrapolation is able to cancel most of the effect of the systematic shift. Figures 7.10 and 7.11 show the Far Detector prediction of the energy spectrum at the input oscillation parameter values together with the fake data energy spectrum. In the ideal case where the extrapolation method completely takes out the effect of the systematic shift, the prediction should line up with the fake data. As expected, the Beam Matrix copes very well with cross-section and beam related uncertainties.

According to the results in table 7.2, the largest contributions to the systematic uncertainty come from the relative normalisation, the neutral current back- 

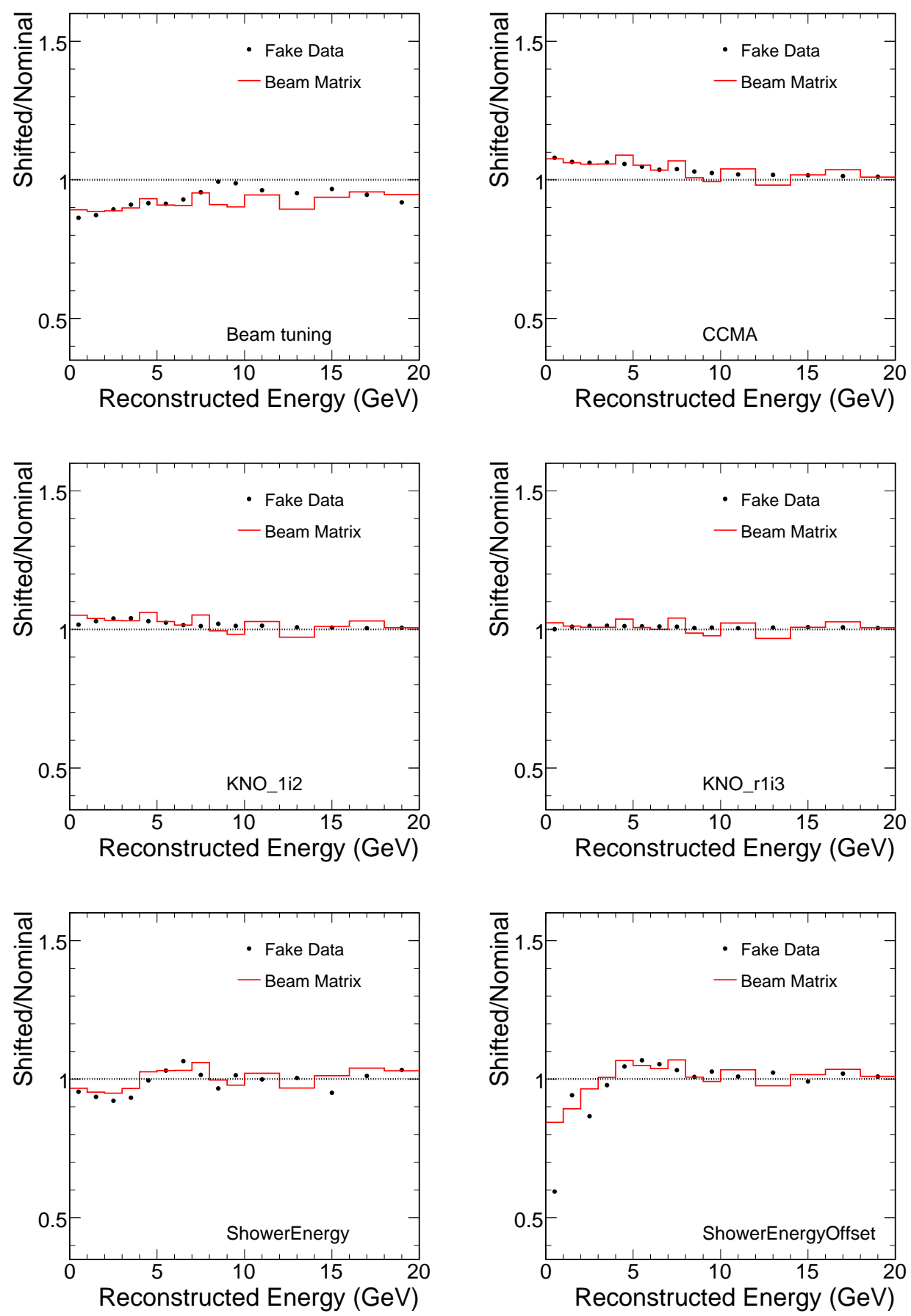

Figure 7.10: Ratio of far detector prediction and fake data. If the Beam Matrix extrapolation was able to cancel a systematic effect perfectly, the red line should align with the fake data markers. 

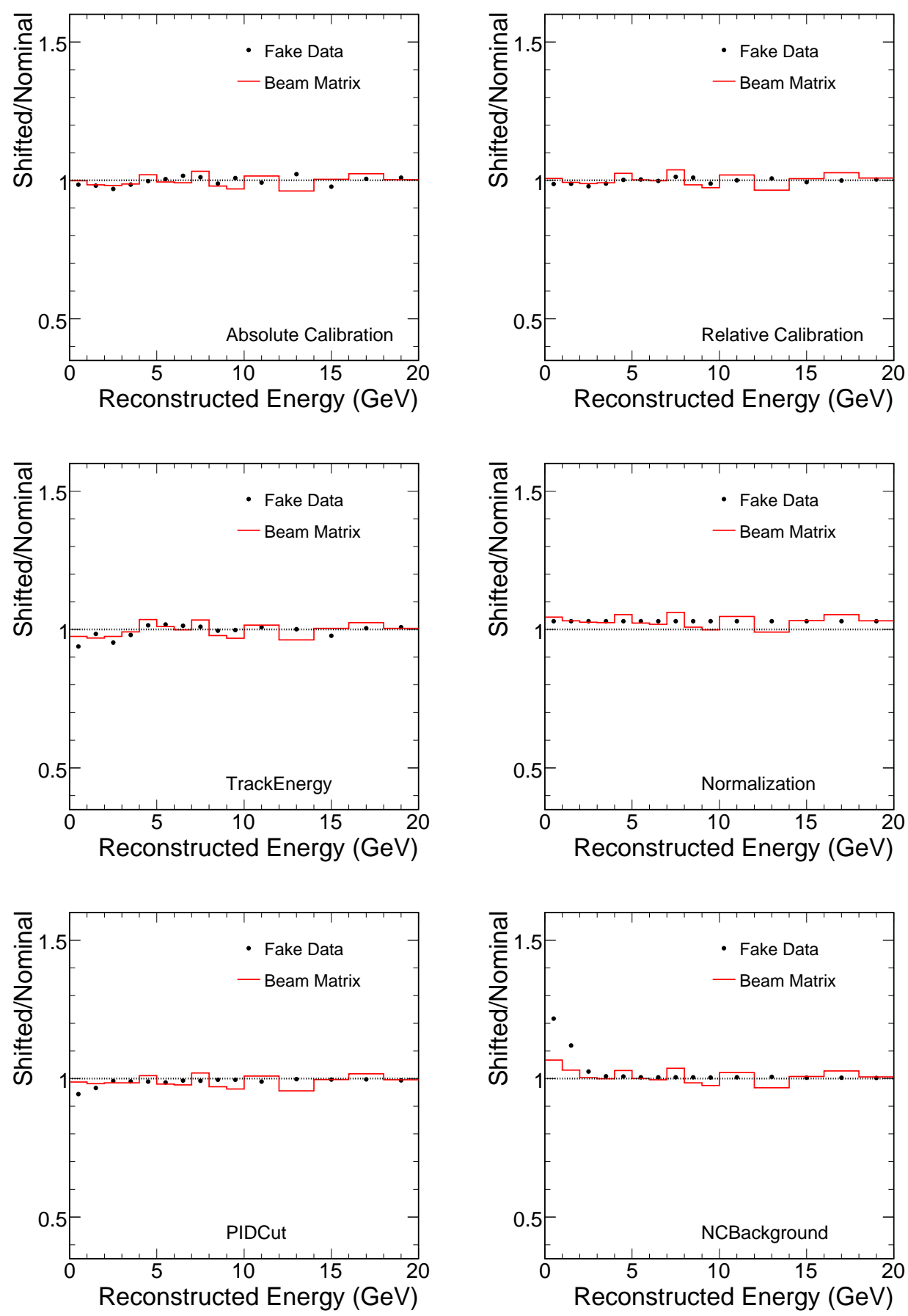

Figure 7.11: Ratio of Far Detector prediction and fake data (cont'd). If the Beam Matrix extrapolation was able to cancel a systematic effect perfectly, the red line should align with the fake data markers. 


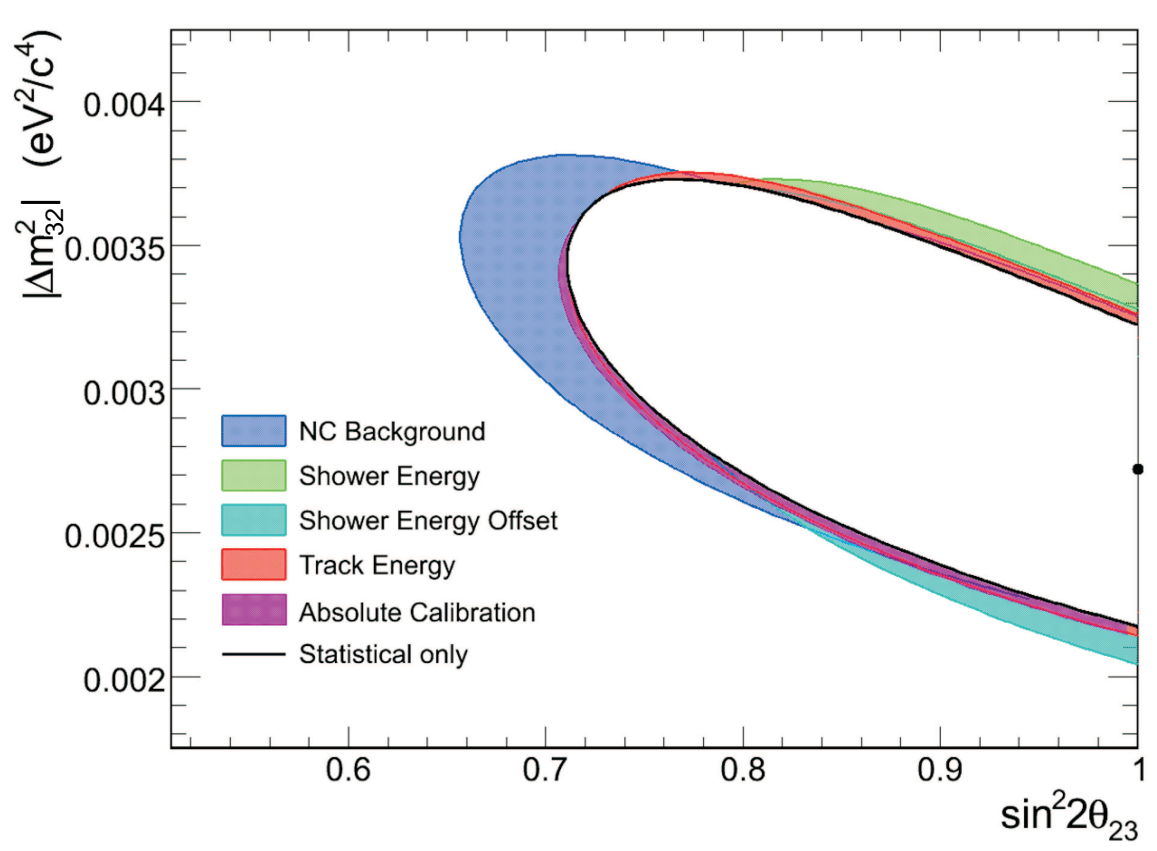

Figure 7.12: Approximate effect of systematic errors on allowed region. The allowed region at $90 \%$ C.L. from a statistics-only fit was moved by the shift in best-fit value caused by different systematic variations. This is an indication of the relative importance of the systematic parameters and shows, which part of the parameters space they are likely to affect.

ground uncertainty and the shower energy scale, where the shower energy scale uncertainties have two distinct sources: calibration uncertainties and the effect of intra-nuclear rescattering.

Figure 7.12 is intended to give a "feeling" about the magnitude of the different systematic shifts on the $90 \%$ C.L. allowed region. To visualise the effect of the individual shifts in the $\left(\Delta m_{32}^{2}, \sin ^{2}\left(2 \theta_{23}\right)\right)$ plane, the allowed region at $90 \%$ C.L. from a statistics only fit was taken and moved by the vector formed by the $\left(\Delta\left(\Delta m_{32}^{2}\right), \Delta\left(\sin ^{2}\left(2 \theta_{23}\right)\right)\right)$-pair. For better visual clarity, only a subset of the systematic parameters is shown. The large contribution of the NC background uncertainty is clearly visible. It also shows how variations to the shower energy scale affect the extremes of the contour in $\Delta m_{32}^{2}$ while having no effect on $\sin ^{2}\left(2 \theta_{23}\right)$.

The results in table 7.2 have to be taken with a degree of caution. This analysis considered each of the systematic variations on its own. It does not take 
into account possible correlations between the different systematic parameters. The averaging of the positive and negative shifts only considered the magnitude of the resulting effect, not its sign. Finally, only the shift of the best-fit value was considered. The effect on the allowed region in parameter space can be different from a simple shift. For these reasons, the results of the fake data study were not used to obtain the final systematic errors on the oscillation parameters.

Instead, the results of table 7.2 and their equivalent for the other proposed extrapolation methods were used to evaluate the performance of the different methods and compare them to each other. The Beam Matrix method was chosen to be the primary extrapolation method on the basis of this study. Furthermore, the results were used to determine the systematic effects which have the biggest impact on the analysis. The three most important systematic effects were subsequently included in the fit by means of nuisance parameters in the $\chi^{2}$ expression (7.6).

\subsubsection{Including systematic errors in the fit}

As shown in the previous section, the main contributions to the systematic uncertainties of this measurement are: the neutral current background, the relative normalisation and the various contributions to the shower energy scale. In order to simplify the fit and to make sure not to overestimate the effect, the different contributions to the shower energy scale (absolute and relative calibration, intranuclear rescattering) were combined into one systematic parameter. The final fit therefore included three nuisance parameters $\alpha_{i}$. The $1 \sigma$ errors on the nuisance parameters were: $4 \%$ on the relative normalisation, $50 \%$ on the neutral current background and $11 \%$ on the shower energy scale. The impact of these nuisance parameters on the allowed region in parameter space is shown in Figure 7.13.

The combined statistical and systematic errors on the oscillation parameters 


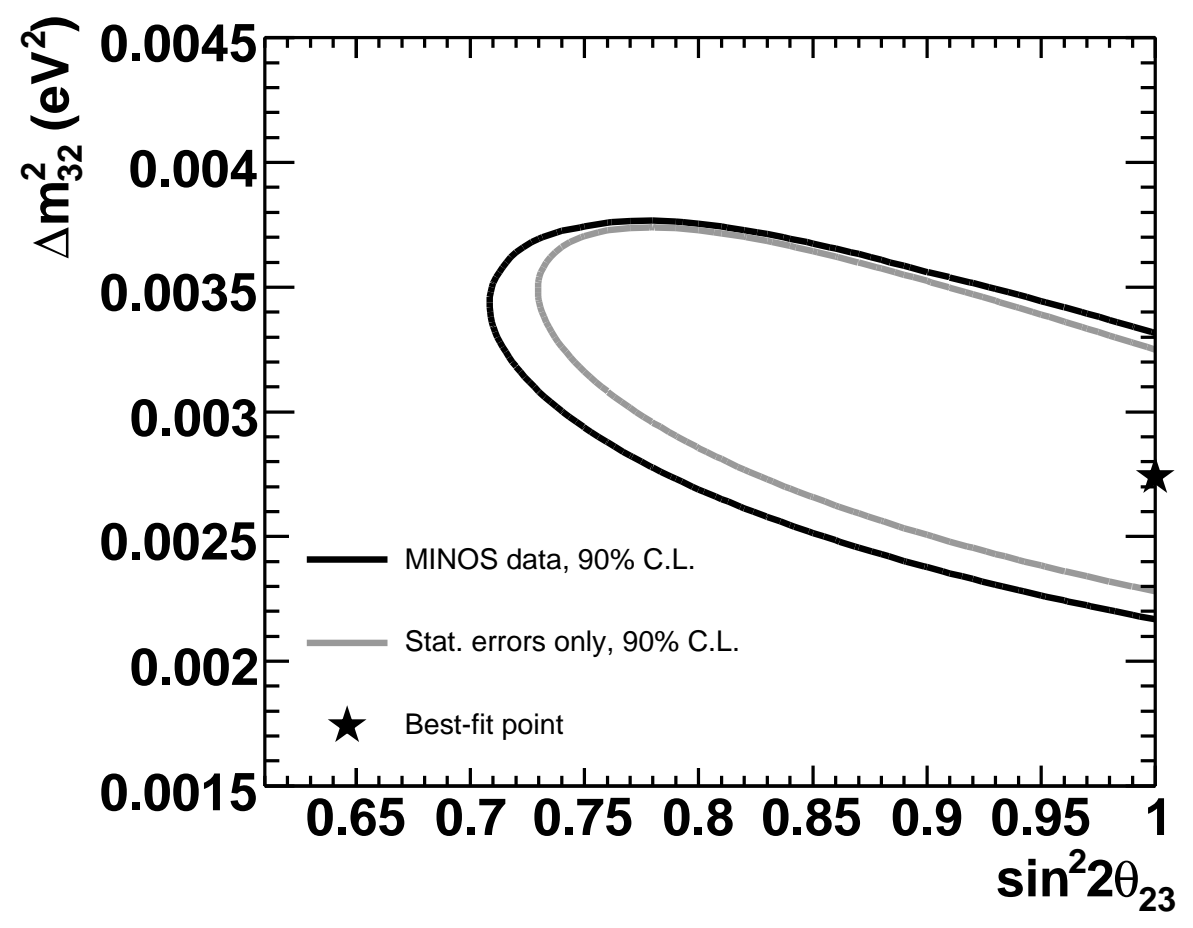

Figure 7.13: Allowed regions at $90 \%$ C.L. with and without including systematic errors in the $\chi^{2}$ expression. The effect of the systematic errors is qualitatively similar to the simplified treatment in Figure 7.12. The star shows the best-fit point at $\Delta m_{32}^{2}=2.74 \times 10^{-3} \mathrm{eV}^{2}$ and $\sin ^{2}\left(2 \theta_{23}\right)=1.0$. 
are then found from the 1-dimensional projections of the $\chi^{2}$ surface. The $68 \%$ C.L. errors correspond to a rise in the $\chi^{2}$ value of 1 unit.

\subsection{Conclusion}

In this chapter, a complete charged current $\nu_{\mu}$ disappearance analysis was presented. The methods used for Monte Carlo tuning, charged current event selection, Near to Far Detector extrapolation, and fit for oscillations were described in detail, with special emphasis on the evaluation of systematic uncertainties. The resulting oscillation parameters for the data collected during the first run period were presented. 


\section{Chapter 8}

\section{Conclusion and Outlook}

MINOS is one of the most exciting neutrino experiments currently operating. Since starting beam operations in early 2005, MINOS has recorded around $3 \times$ $10^{20}$ protons-on-target. Both MINOS detectors as well as the NuMI beamline are performing exceptionally well. The first results of the flagship analysis of MINOS, the charged current muon neutrino disappearance measurement, have been published in Phys. Rev. Lett. in the autumn of 2006. An update on this analysis with more statistics as well as analyses on other, more exotic topics are eagerly awaited by the neutrino physics community.

This thesis addressed a large part of one of these future analyses: the analysis of neutral current interactions. Neutral current interactions are interesting for multiple reasons. Their cross-sections are poorly known in the energy ranges relevant to MINOS. More importantly, they help distinguish between the "standard neutrino physics model" and more exotic phenomena. Is the oscillation we measure in MINOS purely $\nu_{\mu} \rightarrow \nu_{\tau}$ or are there oscillations into sterile neutrinos? In case of the latter, what is the fraction of transitions into sterile neutrinos? The analysis of neutral current interactions will help to answer these questions.

Most of the steps towards a full neutral current analysis were presented in this thesis. A complete, preliminary study on simulated data was conducted, 
including the selection of neutral current events, Near Detector to Far Detector extrapolation and a maximum likelihood fit for oscillations into sterile neutrinos.

The neutral current event selection was subsequently expanded and improved. A set of cuts identifying badly reconstructed events caused by the high rate of interactions in the Near Detector was developed and tested on Monte Carlo and data. Two separate neutral current event selection methods, one using a simple succession of cuts and another using artificial neural networks, were developed, compared and contrasted. As expected, the more advanced neural network techniques has a slightly better performance.

The so selected neutral current interactions in the Near Detector were used to measure cross-section scale factors in four ranges of true neutrino energy. A maximum likelihood fit to data recorded in three different beam configurations was developed for this study. Finally, the systematic uncertainties were evaluated and discussed in detail. The measured cross-section factors are consistent with unity.

The final chapter of this thesis discussed the analysis of charged current interactions in MINOS. This analysis has lead to the first MINOS publication using beam neutrinos in the autumn of 2006. We described a PDF-based selection procedure, a Near to Far Detector extrapolation method using a series of matrices and the final oscillation fit to the data from the first running period. Special attention was devoted to the evaluation and discussion of systematic uncertainties for this measurement.

The near future will undoubtedly see many more exciting results from MINOS. The coming months will see an update to the main charged current analysis with approximately twice the statistics compared to what was presented here. The neutral current analysis is nearly done. It will be concluded by applying the results of chapter 6 to the prediction of the neutral current energy spectrum in the Far Detector and fitting for oscillations to sterile neutrinos. First preliminary 
results from this analysis are expected in the autumn of this year.

Finally, it is worth pointing out that there are many other analyses in progress in MINOS which could not all be described in this thesis. Different analysis working groups are investigating such varied topics as: neutrino-to-anti-neutrino transitions, coherent pion production in the Near Detector, and, maybe most importantly, electron-neutrino appearance in the Far Detector.

MINOS has already made an important impact on the field of neutrino physics. The characteristic energy dependent $\nu_{\mu}$ disappearance, consistent with neutrino oscillations, has been measured with high precision in an accelerator based neutrino experiment. The next charged current result, with more statistics, will be able to rule out some more exotic, competing theories such as neutrino decay or neutrino decoherence. And the search for electron-neutrino appearance will be able to improve on the CHOOZ limit for the third mixing angle $\theta_{13}$ or maybe even measure a non-zero value. That would probably be the most exciting result of all for MINOS. If not, the future experiments $\mathrm{T} 2 \mathrm{~K}, \mathrm{NO} \nu \mathrm{A}$ and Double Chooz may answer this question.

Neutrino physics has resulted in some of the most exciting and surprising discoveries in the past half century. However, there is still a lot to be done. Whatever nature has in stock for us, there are exciting times ahead! 


\section{Appendix A}

\section{Optimising electronics thresholds in the Far Detector}

\section{A.1 Far Detector front-end electronics}

The central part of the MINOS Far Detector front-end electronics is the VA Frontend Board (VFB; see Figure A.1). It houses three custom-designed multi-channel VA chips [77] with a charge-sensitive preamplifier, shaper and sample \& hold for each channel, followed by an analogue output multiplexer. The output signal of the VA chips is then routed to a VA Readout Controller (VARC) in a VME crate where digitisation and time-stamping take place.

Each VA channel input goes to a charge-sensitive preamplifier followed by a shaper, which shapes the signal into a unipolar pulse. The peak occurs after approximately $500 \mathrm{~ns}$. If the PMT signal passed the trigger threshold (see below), the VARC generates a correctly timed hold signal to sample the peak charge.

The triggering of the readout is based on the common dynode signal of the multi-anode PMTs. The M16 multi-anode PMT effectively consists of 16 independently read out photomultipliers (referred to as pixels) in one housing. The trigger signal is generated by the last dynode before the anode. The dynode array 


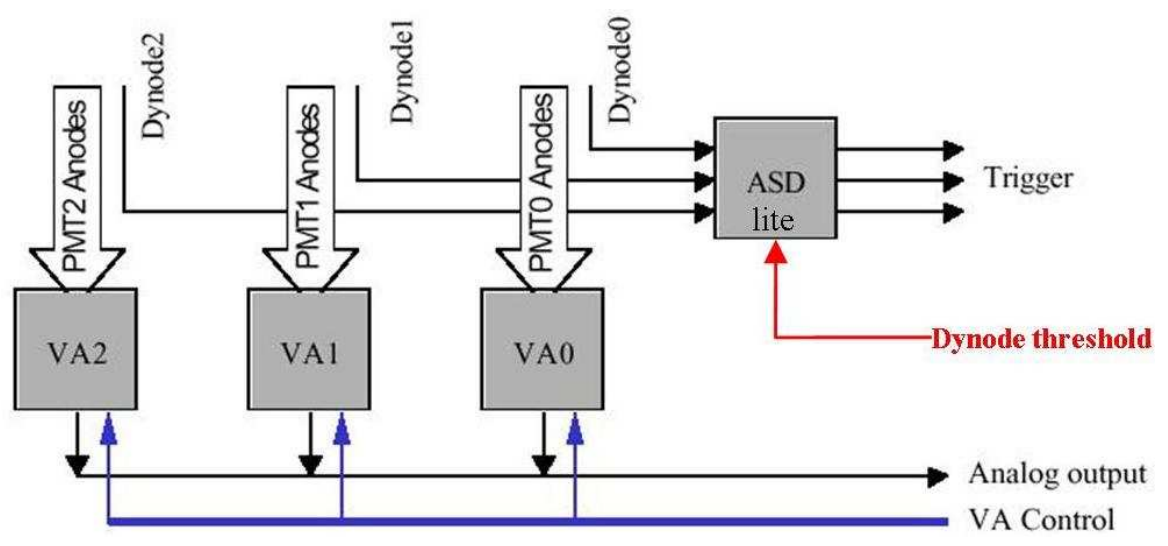

Figure A.1: The VA Front-end Board (VFB). The dynode threshold is set by a DAC on the ASD lite chip, which receives the signals from the PMTs' common dynodes.

of the different pixels is connected to the same resistor chain; therefore, each PMT provides a single trigger signal common to all of its anodes.

The threshold for this trigger is set by a digital-to-analogue-converter (DAC) in a chip, called ASD lite, on the VFB (see Figure A.1). It is identical for all three PMTs connected to the board. The optimisation of these thresholds for the whole detector is described in the following section.

\section{A.2 Optimising the dynode thresholds}

\section{A.2.1 Motivation for the tuning}

The dynode threshold determines whether a signal in the PMT is 'interesting', that is to say large enough to be digitised and subsequently stored by the data acquisition system. Signals that do not cross this threshold are not 'seen' by the detector, and are therefore lost to physics analyses. It is immediately clear that the setting of this threshold is crucial to the performance of the detector. If the dynode threshold is set too high, valid data hits in the detector will be lost. This can deform the topology of an event and introduce a bias on the reconstructed 

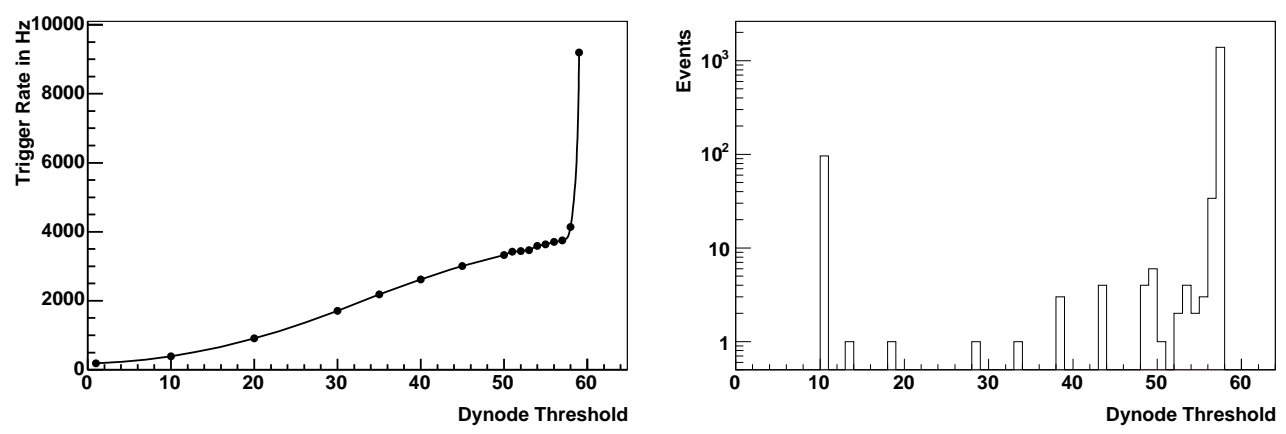

Figure A.2: (a) Trigger rate vs. dynode threshold for one PMT, (b) Distribution of the determined dynode thresholds for all PMTs. 10 is the setting for shield modules, which have a very high rate and 52-57 are the most common settings for normal PMTs. The intermediate values are caused by hardware malfunctions. The spacing in multiples of five is caused by the spacing of our data points at high dynode thresholds.

energy of the event. If set too low, however, the readout will be triggered by noise in the PMTs, resulting in a high proportion of dead-time in the corresponding VA chip.

\section{A.2.2 A special run sequence: the dynode scan}

The VFB allows the dynode threshold to be set between 0 and 63 , where 0 corresponds to the highest and 63 to the lowest possible setting. Prior to this study, the dynode thresholds were set to a default value of 57 throughout the Far Detector.

In order to find the optimum dynode threshold for a particular VFB, a special run sequence, subsequently called 'dynode scan', was devised. It consists of approximately 240 physics runs, each about $10 \mathrm{~s}$ long. In these runs about 20 different dynode thresholds are applied and only one channel per VA is read out. The other channels are deactivated, thus not contributing to the data output. This way, one hit per trigger is recorded. It is important to note that the data acquisition system in the MINOS Far Detector was not designed to handle large data rates. In order to decrease the load on the DAQ, only $1 / 12$ of the detector was active in any given run of the dynode scan sequence, increasing the total number 
of runs to 240 .

The data acquired in this run sequence was subsequently used to investigate the correspondence between dynode threshold setting and trigger rate. Figure A.2(a) shows a typical pattern found in most PMTs.

As one moves to the right on the $x$-axis, the dynode threshold decreases (higher values correspond to a lower threshold). Consequently, the trigger rate rises. At a setting of about 58 , the trigger rate shoots up, thus indicating that noise is causing the trigger signal. It is straightforward to find the optimum trigger threshold in cases like this. Here a value of 56 has been chosen.

As part of this project, we developed an algorithm which automatically determines the threshold setting for each PMT. In principle, this algorithm looks for the setting where the trigger rate rises by more than 10 per cent in one step and then sets the threshold one DAC count below this setting. The distribution of dynode thresholds obtained with this algorithm is shown in Figure A.2(b). The peak at 10 is caused by veto shield modules which sit at the outside of the detector and which therefore have a very high rate due to radioactivity in the surrounding rock. They are set to a fixed threshold of 10 by default. The vast majority of PMTs has a dynode threshold in the region between 52 and 57. The few intermediate values are caused by hardware malfunctions.

There is, however, only one dynode threshold setting per VFB. After determining the optimum threshold for each PMT, the highest threshold of the three PMTs connected to a given VFB is set for this VFB. This way we make sure that none of the VA chips are triggered by noise and therefore dead to the readout for a substantial amount of the time. 

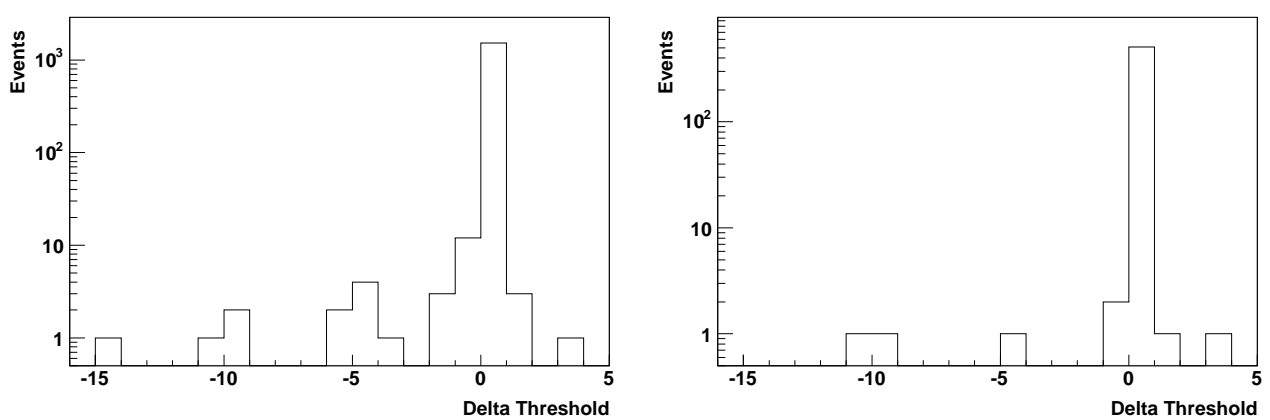

Figure A.3: Difference in determined dynode thresholds for two dynode scans. The spacing in multiples of five is due to the spacing of our data points. (a) difference for all PMTs, (b) difference for all VFBs

\section{A.2.3 Stability of the procedure}

After this procedure was developed, two dynode scans of the whole Far Detector were taken with a separation of about one week. In order to gain a measure of the stability of this procedure for determining the optimum dynode thresholds, the difference between the resulting thresholds for each PMT were calculated. Results of this are shown in Figure A.3.

The distributions are nicely centred at zero and have a width of less than one setting. Note that the y axis has a log scale! The left plot shows the difference of determined threshold for all PMTs. The plot on the right shows the difference between the threshold determined for each VFB as explained above. All outliers of these distributions have been investigated and are well understood. They are all the result of broken or misbehaving hardware. In these cases the algorithm cannot properly determine a valid threshold. Instead, it returns an error flag to alert the user of a possible hardware problem.

\section{A.2.4 Dynode threshold to ADC correspondence}

When considering changing the dynode thresholds, especially when dealing with a particularly noisy PMT, it is necessary to know what a dynode threshold setting 
corresponds to in terms of ADC counts or even in number of photoelectrons. For certain analyses it might be important to detect weak signals with a magnitude of one photoelectron and even the lowest threshold above the pedestal will cut into the single photoelectron peak.

In order to establish this correspondence, a few short runs of type 'singles run' with various different dynode thresholds have been taken. 'Singles run' refers to a run without any additional triggers such as the requirement for hits in at least two detector planes, as required in a normal physics run. In a 'singles run' the dominant contribution to the signal is thus caused by single photons ('singles') spontaneously created in the wavelength shifting fibres or due to radioactivity. The influence of varying the dynode threshold on the ADC spectrum of one PMT is shown in Figure A.4. From top left to bottom right, these thresholds are: 40, 20, 10 and 1 . The threshold of the distribution corresponds to the dynode
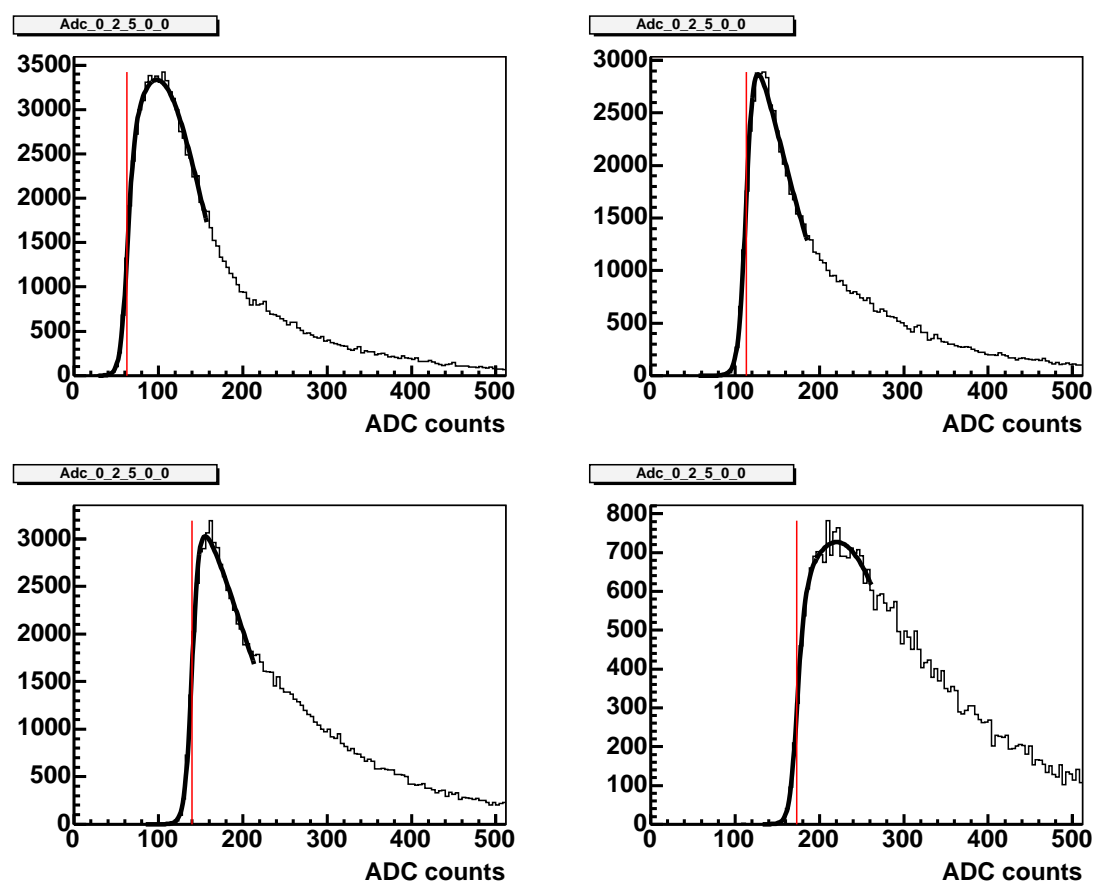

Figure A.4: Singles ADC spectra of one PMT for different dynode threshold settings. The threshold of the distribution increases with increasing dynode threshold, as more and more of the distribution is cut away. 

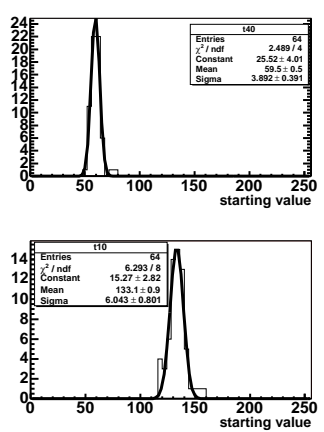
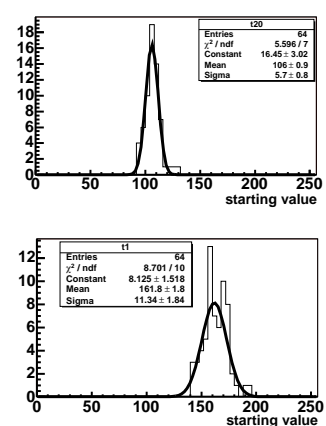

(a)

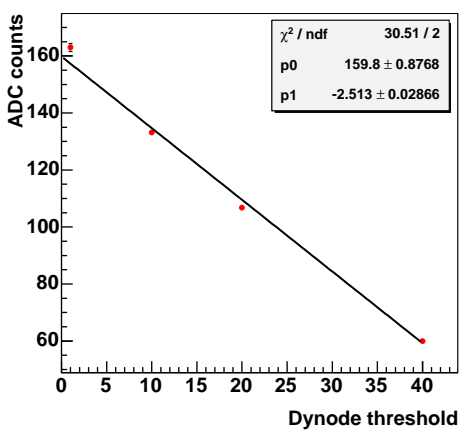

(b)

Figure A.5: (a) Distributions of fitted threshold for all PMTs with high enough statistics, (b) ADC to dynode threshold correspondence

threshold. In order to determine this value, the product of a Gaussian and a Fermi step function has been fitted to the low ADC region of each distribution. The following formula was used:

$$
\frac{1}{1+e^{\frac{x-t}{\lambda}}} \times N e^{-\frac{(x-\mu)^{2}}{2 \sigma^{2}}}
$$

The parameter $t$ in the Fermi function corresponds to the threshold which is to be determined. $\mu$ and $\sigma$ are the mean and width of the Gaussian which describes the dark spectrum of the PMTs and $N$ is an overall normalisation constant.

The singles runs taken for this analysis are very short, so as not to block the DAQ from actual data taking. Therefore only a few PMTs had enough hits to be useful for this analysis. Distributions of the fitted thresholds for all these PMTs and for the four different thresholds are shown in Figure A.5(a).

As a final step we plot the mean of these distributions against the dynodes threshold setting and apply a linear fit (see Figure A.5(b)). The error bars in the plot correspond to the error on the mean of the distributions in Figure A.5(a).

This procedure produces the following results: One step in dynode threshold setting corresponds to about 2.5 ADC counts; the DAC which sets the threshold is not really linear. The latter fact can be seen from the bad $\chi^{2} /$ dof value of 
the straight line fit. This means if the dynode threshold on a particular VFB is increased by for example two settings in order to get rid of noise, about 5 ADC counts are cut from from the signal. To put this into perspective, consider the position of the single photoelectron peak at about 60 ADC counts. By increasing the threshold to, for example, a setting of 40, we would lose $50 \%$ of the single photoelectron signal.

\section{A.3 Summary}

The setting of the dynode thresholds in the MINOS Far Detector is an important part of optimising the detector performance. The impact of changing the dynode thresholds on trigger rates and dead-time has been investigated and optimum dynode threshold settings for the whole detector have been determined.

Furthermore, the correspondence between dynode settings and ADC counts has been established, thus allowing to estimate the impact of higher thresholds on the physics analyses to be done with the MINOS data. It has been shown that increasing the threshold by a few settings can reduce noise levels and dead-times significantly without any adverse effects on the physics capabilities. 


\section{Bibliography}

[1] W. Pauli, Letter to a physicists' gathering at Tubingen. December 4, 1930, reprinted in Wolfgang Pauli, Collected scientific papers, edited by R. Kronig and V. Weisskopf, vol. 2, p. 1313, New York, Interscience, 1964.

[2] J. Chadwick, Possible existence of a neutron, Nature 129, 312 (1932).

[3] E. Fermi, An attempt of a theory of beta radiation. 1, Z. Phys. 88, 161 (1934).

[4] H. Bethe and R. Peierls, The 'neutrino', Nature 133, 532 (1934).

[5] F. Reines and C. L. Cowan, The neutrino, Nature 178, 446 (1956).

[6] M. Goldhaber, L. Grodzins, and A. W. Sunyar, Helicity of neutrinos, Phys. Rev. 109, 1015 (1958).

[7] R. Davis and D. S. Harmer, Attempt to Observe the $\mathrm{Cl}^{37}\left(\bar{\nu}, e^{-}\right) \mathrm{Ar}^{37}$ Reaction Induced by Reactor Antineutrinos, Bull. Am. Phys. Soc. 4, 217 (1959).

[8] G. Danby et al., Observation of high-energy neutrino reactions and the existence of two kinds of neutrinos, Phys. Rev. Lett. 9, 36 (1962).

[9] E598 Collaboration: J. J. Aubert et al., Experimental Observation of a Heavy Particle J, Phys. Rev. Lett. 33, 1404 (1974).

[10] SLAC-SP-017 Collaboration: J. E. Augustin et al., Discovery of a Narrow Resonance in e+ e- Annihilation, Phys. Rev. Lett. 33, 1406 (1974). 
[11] Gargamelle Neutrino Collaboration: F. J. Hasert et al., Observation of neutrino-like interactions without muon or electron in the Gargamelle neutrino experiment, Phys. Lett. B46, 138 (1973).

[12] R. Davis, D. S. Harmer, and K. C. Hoffman, Search for neutrinos from the sun, Phys. Rev. Lett. 20, 1205 (1968).

[13] GALLEX Collaboration: W. Hampel et al., GALLEX solar neutrino observations: Results for GALLEX IV, Phys. Lett. B447, 127 (1999).

[14] D. N. Abdurashitov et al., The Russian-American gallium experiment (SAGE) Cr neutrino source measurement, Phys. Rev. Lett. 77, 4708 (1996).

[15] H. Ikeda et al., Kamiokande: The Kamioka Nucleon Decay Experiment, UTLICEPP-82-04 (1982).

[16] S. P. Mikheev and A. Y. Smirnov, Resonance enhancement of oscillations in matter and solar neutrino spectroscopy, Sov. J. Nucl. Phys. 42, 913 (1985).

[17] L. Wolfenstein, Neutrino oscillations in matter, Phys. Rev. D17, 2369 (1978).

[18] Irvine-Michigan-Brookhaven Collaboration: R. M. Bionta et al., A Search for Proton Decay Into e+ pi0, Phys. Rev. Lett. 51, 27 (1983).

[19] ALEPH Collaboration: D. Decamp et al., Determination of the Number of Light Neutrino Species, Phys. Lett. B231, 519 (1989).

[20] Delphi Collaboration: P. A. Aarnio et al., Measurement of the Mass and Width of the Z0 Particle from Multi - Hadronic Final States Produced in e+ e- Annihilations, Phys. Lett. B231, 539 (1989).

[21] L3 Collaboration: B. Adeva et al., A Determination of the Properties of the Neutral Intermediate Vector Boson Z0, Phys. Lett. B231, 509 (1989). 
[22] OPAL Collaboration: M. Z. Akrawy et al., Measurement of the Z0 Mass and Width with the OPAL Detector at LEP, Phys. Lett. B231, 530 (1989).

[23] DONUT Collaboration: T. Patzak, First direct observation of the tauneutrino, Europhys. News 32, 56 (2001).

[24] Super-Kamiokande Collaboration: Y. Fukuda et al., Evidence for oscillation of atmospheric neutrinos, Phys. Rev. Lett. 81, 1562 (1998).

[25] SNO Collaboration: Q. R. Ahmad et al., Direct evidence for neutrino flavor transformation from neutral-current interactions in the Sudbury Neutrino Observatory, Phys. Rev. Lett. 89, 011301 (2002).

[26] KamLAND Collaboration: K. Eguchi et al., First results from KamLAND: Evidence for reactor anti- neutrino disappearance, Phys. Rev. Lett. 90, $021802(2003)$.

[27] K2K Collaboration: M. H. Ahn et al., Measurement of neutrino oscillation by the K2K experiment, Phys. Rev. D74, 072003 (2006).

[28] MINOS Collaboration: D. G. Michael et al., Observation of Muon Neutrino Disappearance with the MINOS Detectors in the NuMI Neutrino Beam, Phys. Rev. Lett. 97, 191801 (2006).

[29] B. Pontecorvo, Inverse beta processes and nonconservation of lepton charge, Sov. Phys. JETP 7, 172 (1958).

[30] Z. Maki, M. Nakagawa, and S. Sakata, Remarks on the unified model of elementary particles, Prog. Theor. Phys. 28, 870 (1962).

[31] B. Kayser, On the Quantum Mechanics of Neutrino Oscillation, Phys. Rev. D24, 110 (1981). 
[32] M. Kobayashi and T. Maskawa, CP Violation in the Renormalizable Theory of Weak Interaction, Prog. Theor. Phys. 49, 652 (1973).

[33] A. Faessler and F. Simkovic, Double beta decay, J. Phys. G24, 2139 (1998).

[34] G. L. Fogli, E. Lisi, and G. Scioscia, Accelerator and reactor neutrino oscillation experiments in a simple three generation framework, Phys. Rev. D52, 5334 (1995).

[35] B. Richter, Conventional beams or neutrino factories: The next generation of accelerator-based neutrino experiments, hep-ph/0008222 (2000).

[36] A. De Santo, An experimentalist's view of neutrino oscillations, Int. J. Mod. Phys. A16, 4085 (2001).

[37] The ALEPH, DELPHI, L3, OPAL, SLD Collaborations, the LEP Electroweak Working Group, the SLD Electroweak and Heavy Flavour Groups, Precision Electroweak Measurements on the Z Resonance, Phys. Rept. 427, $257(2006)$.

[38] LSND Collaboration: A. Aguilar et al., Evidence for neutrino oscillations from the observation of anti-nu/e appearance in a anti-nu/mu beam, Phys. Rev. D64, 112007 (2001).

[39] The MiniBooNE Collaboration: A. A. Aguilar-Arevalo et al., A search for electron neutrino appearance at the $\Delta m^{2} \sim 1 \mathrm{eV}^{2}$ scale, arXiv:0704.1500 [hep-ex] (2007).

[40] R. P. Litchfield, Neutrino induced events in the MINOS detectors, DPhil thesis, University of Oxford, 2007.

[41] W.-M. Yao et al., Review of Particle Physics, Journal of Physics G: Nuclear and Particle Physics 33, 1 (2006). 
[42] J. N. Bahcall, A. M. Serenelli, and S. Basu, New solar opacities, abundances, helioseismology, and neutrino fluxes, Astrophys. J. 621, L85 (2005).

[43] Structure and Dynamics of the Interior of the Sun and Sun-like Stars, in ESA Special Publication, edited by S. Korzennik volume 418 of ESA Special Publication, 1998.

[44] Kamiokande-II Collaboration: K. S. Hirata et al., Real time, directional measurement of B-8 solar neutrinos in the Kamiokande-II detector, Phys. Rev. D44, 2241 (1991).

[45] Super-Kamiokande Collaboration: Y. Fukuda et al., Constraints on neutrino oscillation parameters from the measurement of day-night solar neutrino fluxes at Super- Kamiokande, Phys. Rev. Lett. 82, 1810 (1999).

[46] P. A. Cerenkov, Visible radiation produced by electrons moving in a medium with velocities exceeding that of light, Phys. Rev. 52, 378 (1937).

[47] SNO Collaboration: B. Aharmim et al., Electron energy spectra, fluxes, and day-night asymmetries of B-8 solar neutrinos from the 391-day salt phase SNO data set, Phys. Rev. C72 (2005).

[48] KamLAND Collaboration: T. Araki et al., Measurement of neutrino oscillation with KamLAND: Evidence of spectral distortion, Phys. Rev. Lett. 94, $081801(2005)$.

[49] V. D. Barger, J. G. Learned, S. Pakvasa, and T. J. Weiler, Neutrino decay as an explanation of atmospheric neutrino observations, Phys. Rev. Lett. 82, 2640 (1999).

[50] E. Lisi, A. Marrone, and D. Montanino, Probing possible decoherence effects in atmospheric neutrino oscillations, Phys. Rev. Lett. 85, 1166 (2000). 
[51] T. Kajita and Y. Totsuka, Observation of atmospheric neutrinos, Rev. Mod. Phys. 73, 85 (2001).

[52] T. K. Gaisser and M. Honda, Flux of atmospheric neutrinos, Ann. Rev. Nucl. Part. Sci. 52, 153 (2002).

[53] T. J. Haines et al., Calculation of atmospheric neutrino induced backgrounds in a nucleon decay search, Phys. Rev. Lett. 57, 1986 (1986).

[54] W. A. Mann, Atmospheric neutrinos and the oscillations bonanza, Int. J. Mod. Phys. A15S1, 229 (2000).

[55] Super-Kamiokande Collaboration: C. Yanagisawa, Recent results from Super-Kamiokande, in Frontiers in Contemporary Physics 2001, Vanderbilt University, Nashville, Tennessee, USA (2001).

[56] Super-Kamiokande Collaboration: K. Okumura, Super-Kamiokande atmospheric neutrino results, Czech. J. Phys. 56, A271 (2006).

[57] Super-Kamiokande Collaboration: Y. Ashie et al., Evidence for an oscillatory signature in atmospheric neutrino oscillation, Phys. Rev. Lett. 93, $101801(2004)$.

[58] Soudan 2 Collaboration: M. C. Sanchez et al., Observation of atmospheric neutrino oscillations in Soudan 2, Phys. Rev. D68, 113004 (2003).

[59] MACRO Collaboration: M. Ambrosio et al., Matter effects in upward-going muons and sterile neutrino oscillations, Phys. Lett. B517, 59 (2001).

[60] M. Maltoni and T. Schwetz, Sterile neutrino oscillations after first MiniBooNE results, arXiv:0705.0107 [hep-ph] (2007).

[61] M. Apollonio et al., Search for neutrino oscillations on a long base-line at the CHOOZ nuclear power station, Eur. Phys. J. C27, 331 (2003). 
[62] T. Schwetz, Global fits to neutrino oscillation data, Phys. Scripta T127, 1 (2006).

[63] NOvA Collaboration: D. S. Ayres et al., NOvA proposal to build a 30kiloton off-axis detector to study neutrino oscillations in the Fermilab NuMI beamline, (2004).

[64] Y. Itow et al., The JHF-Kamioka neutrino project, (2001), hep-ex/0106019.

[65] Double Chooz Collaboration: F. Ardellier et al., Double Chooz: A search for the neutrino mixing angle theta(13), hep-ex/0606025 (2006).

[66] MINOS Collaboration, The MINOS Detectors Technical Design Report, NuMI-L-337 (1998).

[67] J. Hylen et al., NuMI Technical Design Handbook, Internal NuMI report (2003).

[68] NOvA Collaboration: D. S. Ayres et al., NOvA proposal to build a 30kiloton off-axis detector to study neutrino oscillations in the Fermilab NuMI beamline, (2004).

[69] R. M. Zwaska, Accelerator Systems and Instrumentation for the NuMI Neutrino Beam, PhD thesis University of Texas at Austin, 2005.

[70] P. Adamson et al., The MINOS calibration detector, Nucl. Instrum. Meth. A556, 119 (2006).

[71] D. Michael et al., The MINOS detectors, Nucl. Instrum. Meth. in preparation.

[72] A. Pla-Dalmau et al., Extruded plastic scintillators for the MINOS calorimeters, Frascati Phys. Ser. 21, 513 (2001). 
[73] C. B. Choudhary and L. Mualem, Technical specifications for the procurement of Wavelength Shifting Fibre, NuMI-L-0589 (2000).

[74] N. Tagg et al., Performance of Hamamatsu 64-anode photomultipliers for use with wavelength-shifting optical fibres, Nucl. Instrum. Meth. A539, 668 (2005).

[75] K. Lang et al., Characterization of 1600 Hamamatsu 16-anode photomultipliers for the MINOS Far detector, Nucl. Instrum. Meth. A545, 852 (2005).

[76] A. Cabrera, Systematic Comparison of the MINOS Near and Far Detector Readout Systems, DPhil thesis, University of Oxford, 2005.

[77] Ideas ASA, PO Box 1, 1330 Fornebu, Norway.

[78] J. Oliver, N. Felt, G. Feldman, A. Lebedev, and R. Lee, Design and performance of the readout system of the MINOS Far Detector, IEEE Trans. Nucl. Sci. 51, 2193 (2004).

[79] S. Avvakumov et al., Spontaneous light emission from fibers in MINOS, Nucl. Instrum. Meth. A545, 145 (2005).

[80] MINOS Collaboration: P. Adamson et al., First observations of separated atmospheric $\nu_{\mu}$ and $\bar{\nu}_{\mu}$ events in the MINOS detector, Phys. Rev. D73, $072002(2006)$.

[81] G. Drake, J. Dawson, and C. Nelson, Overview of the front end electronics for the MINOS Near Detector, NuMI-L-628 (1999).

[82] T. Cundiff et al., The MINOS near detector front end electronics, IEEE Trans. Nucl. Sci. 53, 1347 (2006). 
[83] R. Brun, R. Hagelberg, M. Hansroul, and J. C. Lassalle, GEANT: Simulation Program for Particle Physics experiments. User guide and reference manual, CERN-DD-78-2-REV.

[84] A. Fasso, A. Ferrari, and P. Sala, Electron photon transport in FLUKA: Status, Prepared for International Conference on Advanced Monte Carlo for Radiation Physics, Particle Transport Simulation and Applications (MC 2000), Lisbon, Portugal, 23-26 Oct 2000.

[85] A. Fasso, A. Ferrari, P. R. Sala, and J. Ranft, FLUKA: Status and prospects for hadronic applications, Prepared for International Conference on Advanced Monte Carlo for Radiation Physics, Particle Transport Simulation and Applications (MC 2000), Lisbon, Portugal, 23-26 Oct 2000.

[86] H. Gallagher, The NEUGEN neutrino event generator, Nucl. Phys. Proc. Suppl. 112, 188 (2002).

[87] C. Andreopoulos, The GENIE universal, object-oriented neutrino generator, Nucl. Phys. Proc. Suppl. 159, 217 (2006).

[88] C. Zeitnitz and T. A. Gabriel, The GEANT - CALOR interface and benchmark calculations of ZEUS test calorimeters, Nucl. Instrum. Meth. A349, 106 (1994).

[89] R. Brun and F. Rademakers, ROOT: An object oriented data analysis framework, Nucl. Instrum. Meth. A389, 81 (1997).

[90] F. James and M. Roos, MINUIT - A System for function minimization and analysis of the parameter errors and correlations, Comput. Phys. Commun. 10, $343(1975)$.

[91] R. Pittam, ND Data Cleaning - Veto Regions, MINOS-doc-2488 (2006). 
[92] M. Barker, A. De Santo, and A. Weber, A first look at afterpulsing in R5900-00-M64, NuMI-L-0685 (2000).

[93] T. Raufer, My final look at AfterPulsing, MINOS-doc-1327 (2005).

[94] G. E. Hinton, How Neural Networks Learn from Experience, Scientific American 267, 105 (1992).

[95] R. Bellman, Adaptive Control Processes: A Guided Tour (Princeton University Press, 1961).

[96] D. Rumelhart, G. Hinton, and R. Williams, Learning representations by back-propagating errors, Nature 323, 533 (1986).

[97] C. Bishop, Neural Networks for Pattern Recognition (Clarendon Press, Oxford, 1995).

[98] A. S. Lapedes and R. M. Farber, How Neural Nets Work., in Neural Information Processing Systems, pages 442-456 (1987).

[99] C. G. Broyden, The Convergence of a Class of Double-rank Minimization Algorithms 1. General Considerations, IMA J Appl Math 6, 76 (1970).

[100] N. J. Baker et al., Quasielastic Neutrino Scattering: A Measurement of the Weak Nucleon Axial Vector Form-Factor, Phys. Rev. D23, 2499 (1981).

[101] S. J. Barish et al., Study of Neutrino Interactions in Hydrogen and Deuterium. 1. Description of the Experiment and Study of the Reaction Neutrino $\mathrm{d} \rightarrow$ mu- p p(s), Phys. Rev. D16, 3103 (1977).

[102] Gargamelle Neutrino Propane Collaboration: W. Krenz et al., Experimental study of exclusive one pion production in all neutrino induced neutral current channels, Nucl. Phys. B135, 45 (1978). 
[103] R. Merenyi et al., Determination of pion intranuclear rescattering rates in muon-neutrino Ne versus muon-neutrino D interactions for the atmospheric neutrino flux, Phys. Rev. D45, 743 (1992).

[104] M. Kordosky, Intranuke Reweighting, MINOS-doc-2093 (2006).

[105] S. Kopp, Z. Pavlovich, and P. Vahle, Effect of Beam Uncertainties on NuMI Analyses, Presentation at NuFact 06, UC Irvine, USA, 24-30 Aug 2006.

[106] A. Para and M. Szleper, Neutrino Spectrum at the Far Detector — Systematic Errors, NuMI-B-0781 (2001).

[107] Super-Kamiokande Collaboration: J. Hosaka et al., Three flavor neutrino oscillation analysis of atmospheric neutrinos in Super-Kamiokande, Phys. Rev. D74, 032002 (2006). 